\title{
ADVANCES IN DEEP BRAIN STIMULATION FOR PARKINSON'S DISEASE
}

\section{PhD Thesis in Biomedical Engineering}

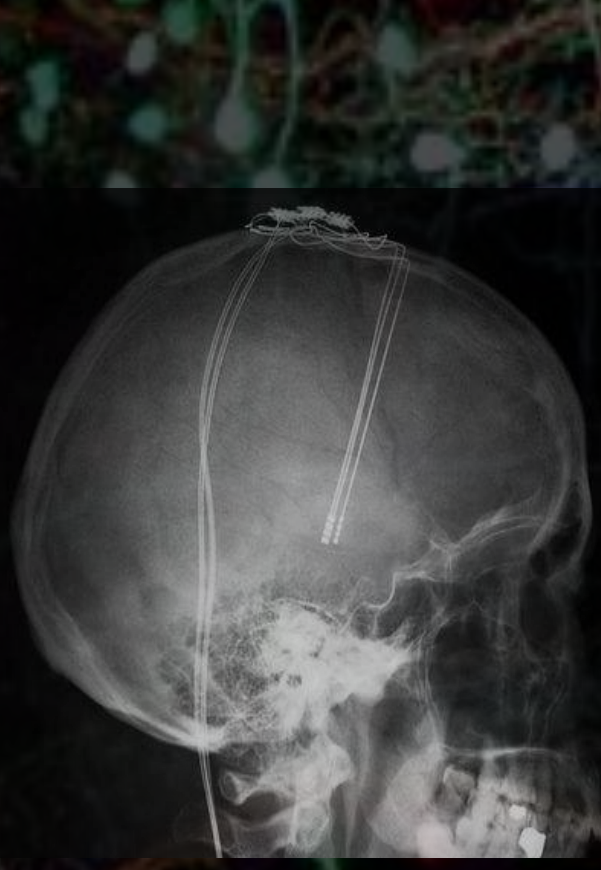

\section{CARMEN CÁMARA}

Kevin Warwick

$\mathrm{PhD}$ in Electrical Engineering
Ernesto Pereda

$\mathrm{PhD}$ in Neuroscience 


\title{
UNIVERSIDAD POLITÉCNICA DE MADRID
}

\section{$\mathrm{PhD}$ in Biomedical Engineering}

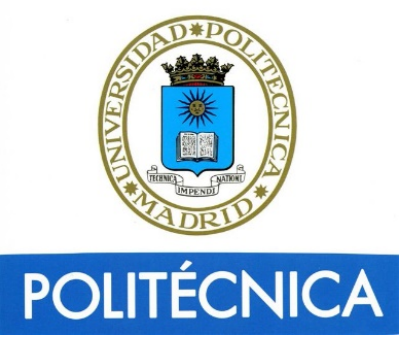

Escuela Técnica Superior de Ingenieros de Telecomunicación (ETSIT UPM) Centre for Biomedical Technology (CTB)

\section{ADVANCES IN DEEP BRAIN STIMULATION FOR PARKINSON'S DISEASE}

\author{
Carmen Cámara Núñez \\ MSc in Biomedical Engineering \\ $\mathrm{PhD}$ in Computer Science
}

Advisors

Kevin Warwick

$\mathrm{PhD}$ in Electrical Engineering
Ernesto Pereda

$\mathrm{PhD}$ in Neuroscience 


\section{Contents}

Acknowledgements

iii

Summary ix

Resumen $\quad$ xi

Acronyms xiii

I Introduction $\quad 1$

1 Introduction to Parkinson's Disease 3

1.1 Brief introduction to the brain . . . . . . . . . . . 4

1.2 Functioning of the basal ganglia. . . . . . . . . . 8

1.3 Pathophysiology of Parkinson's disease. . . . . . . . . . . 10

1.4 Treatments . . . . . . . . . . . . . . . . 16

1.5 Deep brain stimulation . . . . . . . . . . . . . . 17

2 Introduction to Neuroimaging and Analysis Methods 21

2.1 Intracraneal Recordings . . . . . . . . . . . . . . 25

2.2 Magnetoencephalography. . . . . . . . . . . . 26

2.3 Analysis Methods. . . . . . . . . . . . . . . . 31

2.3.1 Spectral Analysis . . . . . . . . . . . . . . 31

2.3.2 Synchronization Analysis . . . . . . . . . . . . . 33

2.3.3 Non-linear Dynamical Analysis . . . . . . . . . . . 37

2.4 Statistical Methods. . . . . . . . . . . . . . . . . . 39

2.4.1 Machine Learning . . . . . . . . . . . . . . . 40

2.4.2 Cluster-based permutation test . . . . . . . . . . 42

II DBS Mistery $\quad 45$

3 DBS effects on cortico-cortical functional connectivity $\quad 47$

3.1 Effects of deep brain stimulation . . . . . . . . . . 48

3.2 Subjects . . . . . . . . . . . . . . . 50

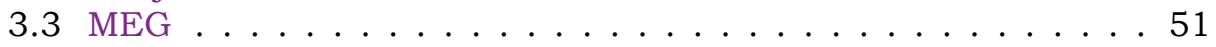

3.4 Analysis . . . . . . . . . . . . . . . . . 53

3.5 Results . . . . . . . . . . . . . . . . . . . 58

3.6 Conclusions . . . . . . . . . . . . . . . . . 63

III Closed-loop DBS $\quad 65$

4 Closed-loop Deep Brain Stimulation $\quad 67$

4.1 Dataset. . . . . . . . . . . . . . . . . . . 71 
5 Resting tremor classification and detection in Parkinson's disease patients $\quad \mathbf{7 5}$

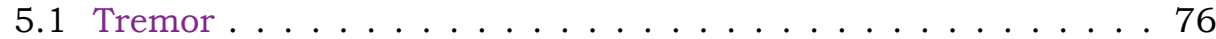

5.2 Data Preparation and feature extraction . . . . . . . . . . 77

5.3 Proposed System \& Results. . . . . . . . . . . . . . . . . 79

5.4 Discussion (Performance Evaluation) . . . . . . . . . . . 88

5.5 Conclusions . . . . . . . . . . . . . . . 88

6 A fuzzy inference system for closed-loop deep brain stimulation in Parkinson's disease $\quad 91$

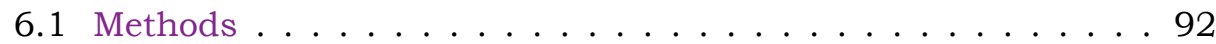

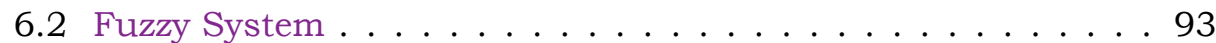

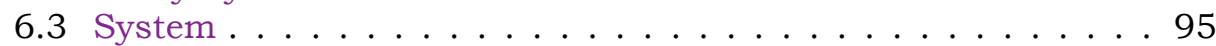

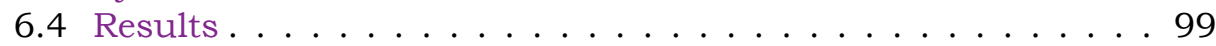

6.5 Discussion. . . . . . . . . . . . . . . . . . . . . . . . . . . . . . . .

6.6 Conclusions. . . . . . . . . . . . . . . 103

7 Non-linear dynamical analysis of resting tremor for demanddriven deep brain stimulation $\quad 105$

7.1 Data Preparation . . . . . . . . . . . . . . 106

7.2 Recurrence Networks . . . . . . . . . . . . . . 107

7.3 Network Measures . . . . . . . . . . . . . . . . 109

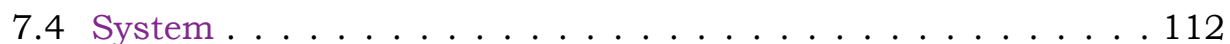

7.5 Discussion. . . . . . . . . . . . . . . . 117

7.6 Conclusions . . . . . . . . . . . . . . . . 119

8 Closed-loop deep brain stimulation based on a stream-clustering system

8.1 Neural oscillations versus synchronization . . . . . . . . . 122

8.2 Signal Preprocessing. . . . . . . . . . . . . . . . 124

8.3 Synchronization analysis . . . . . . . . . . . . . . 124

8.4 Data Stream Mining . . . . . . . . . . . . . . . . 126

8.5 Stream Clustering . . . . . . . . . . . . . . 128

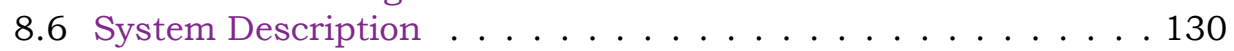

8.7 Experimental Analysis. . . . . . . . . . . . . . . . . . . . . . . . . . 131

8.8 Discussion. . . . . . . . . . . . . . . 138

IV Conclusions \& Future perspectives 143

9 General Conclusions \& Future Perspectives 145

$\begin{array}{ll}\text { Curriculum Vitæ } & 151\end{array}$

Bibliography 153 


\section{Acknowledgements}

$\mathrm{T}$ is said that having a good first boss (in my case, supervisors) is crucial since 1 one will end up looking like him. If this is true, I have been fortunate. Firstly for the high worth of my tutors, but also with the centre in which this thesis has been developed. Here, I want to thank all the people who have helped me in the developing of this thesis.

\section{Kevin}

I discovered who would later be my supervisor, in a television program of scientific divulgation. It was a program in which the researcher interviewed showed his work to the general public. I was still studying the degree, but I thought, "I hope someday I can do half the impressive things this guy does". When I decided I wanted to do the thesis, I got up the courage (of course thinking he would not answer (because he was a superstar)), and sent an email to Kevin Warwick. But he did reply (I still sometimes flip with it), and he gave me an appointment in Reading, UK, and agreed to supervise my thesis!

Kevin was collaborating with Tipu Aziz, from Oxford University, in the design of intelligent systems for the treatment of Parkinson's disease. So he proposed to me to work on that in my thesis. At the time, I only had a master's degree in Artificial Intelligence. Since then, I have also learned Bioengineering and some Neuroimaging and Neuroscience. It has been a thesis's topic that has led me to learn a little bit of quite a few things. So it turned out to be the perfect research area for the thesis. Why isn't this, in the end, the goal of a thesis, exploration and continuous learning?. So I have to thank you, Kevin, for choosing this topic for me, because it has taken me to wonderful places.

I have not been lucky enough to have Kevin in the same lab as me, nor in the same building, not even in the same country. Despite this, his influence has been inspiring; his work will always be an inspiration to me. Thank you, Kevin, for giving me this opportunity, because everything I have learned, all this adventure, started on that visit to Reading. Thanks to Tipu as well for the time he has dedicated to me and the data he has kindly lent me.

\section{Ernesto}

I met Ernesto at a seminar he taught at the master's in bioengineering at UPM. He is an excellent teacher, so I had no doubt that he would be an excellent thesis tutor as well. Ernesto started supervising me in the middle of the thesis. But it has been long enough to learn from him. Like Kevin, he was not in the same geographical place as me. Maybe we have been able to see each other only once or twice a year. 
But on those occasions, Ernesto sat down and talk to me for a long time to define the work for the next few months. He always has excellent ideas, so talking to him is very motivating. Having a skype with him always serves to renew the desire to do many cool things, so I hope they never finish.

I admire very much your ability to get so much work done and commit so much to the university. I don't know how you get there you have time for so much, but I hope part of your future time may be to keep doing cool things together. Thanks, Ernesto.

\section{Center for Biomedical Technology ++}

I knew the CTB during the master's degree in bioengineering, and I decided it was the best place to enrol my thesis. Many students want to be there, and there is no place for everyone. For that reason, I have to thank here Fernando Maestú for giving the opportunity to me. The CTB is a place full of very talented people. It thus becomes an inspiring place, which encourages the desire to learn. This is thanks to the direction of Fernando, but also of all the members of the lab, so I want to thank here all the students I've coincided with there.

I want to give special thanks to Ricardo Bruña. My teacher of "signals and systems" during the master, my master's final project supervisor, and my friend since then. Talking to him is a little frustrating because you realise all you have left to learn. But he always has time to sit with you in front of a blank sheet of paper and paints you drawings and mathematical formulas so that you understand things at a low level (the way I like it). Everything that I know about signal processing has been taught to me by him. And I am sure he will teach me everything I still have to know. I hope we keep working together a lot; it is the best things about the CTB.

Although he is not a CTB colleague, I would like to thank Narayan here as well. He taught me Chaos Theory and his thesis was part of the basic bibliography of my bedside table (I've read it more times than mine). Thank you for being so impressive and spend time with me, I'll never forget your wonderful tutorials at Aalto.

Finally, thanks to the European Institute of Innovation and Technology (EIT Digital) for choosing me to do my thesis within their elitist doctoral school. It has allowed me to do an infinitely more complete training, with a vision of entrepreneurship and digital world (almost as in silicon valley), which is not possible outside its program. Thanks to the colleagues chosen with me, for the time together during the long training courses. We had a great time, and it was a vital experience that I will never forget.

This thesis has never been merely a goal for me, but the beginning of a path, that I have enjoyed so much (I know this is not what a thesis student typically says, but I swear it is real). I hope to keep learning and doing new, inspiring, challenging and wonderful things with all of you, Kevin, Ernesto and colleagues. Thank you all. 


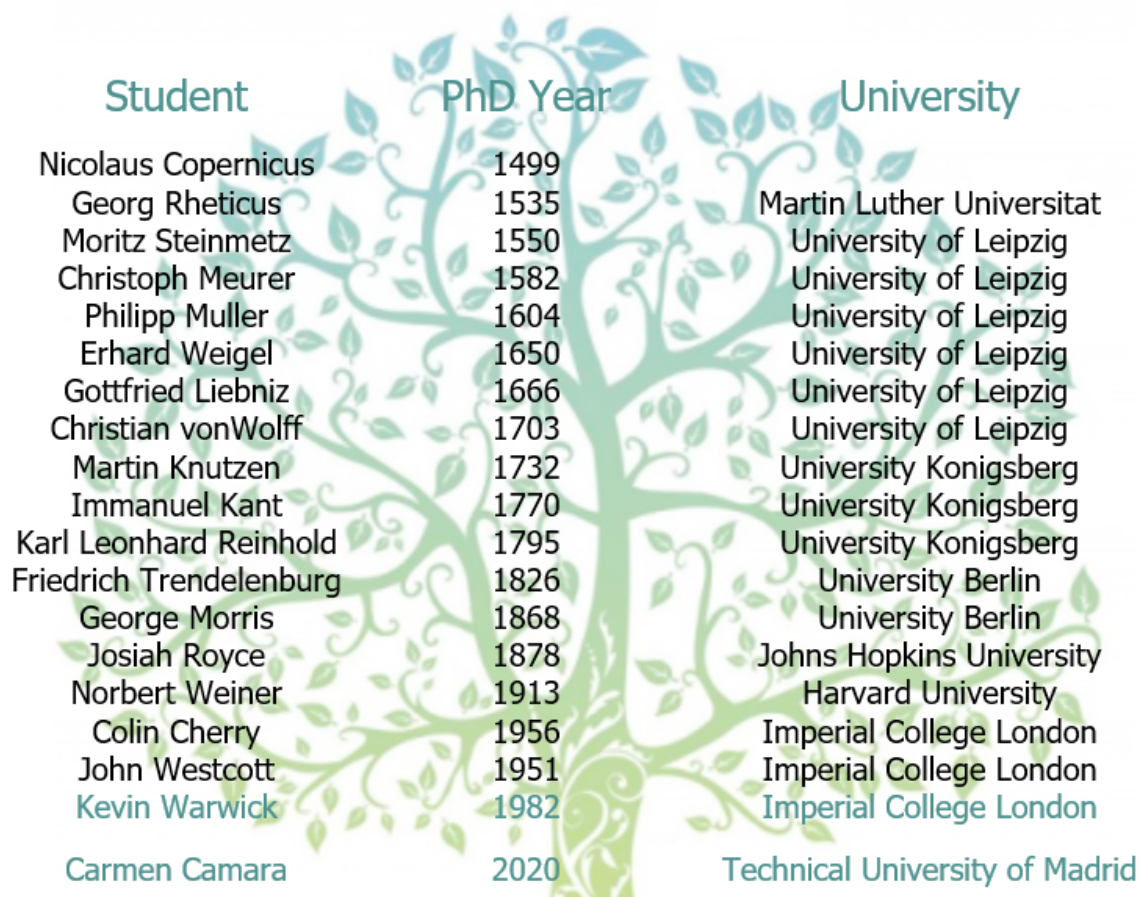




\section{Mi querida familia}

Nada de lo que una persona es o logra es por mérito exclusivo, sino una combinación de toda la educación recibida y una pizca de suerte. Del primer ingrediente, los únicos responsables son mis padres. Siempre nos han dicho que teníamos el potencial para hacer lo que quisieramos y nos han animado a hacerlo desde pequeñas. Convencieron a la directora de mi primer colegio de que podía ir a clases de informatica con 6 años, con estudiantes diez años mayores que yo (quizás porque yo también era muy testaruda). Finalmente pude ir y lo hice bastante bien. Fue mi primer acercamiento a la informática, y desde entonces sabía que quería terminar haciendo cosas como aquellas.

Mi padre es la persona más inteligente que he conocido. Solía pasar la mayor parte del día leyendo, con una curiosidad insaciable. Tenemos más libros en casa que cualquier otro tipo de objeto. Casi todos los domingos nos llevaba a mi hermana y a mí a comprar libros a la feria del libro de Madrid. Nos transfirió esa pasión por el conocimiento. Siempre ha sido (y sigue siendo) una referencia. Mamá es una persona con una gran integridad. Ella siempre nos ha empujado a hacer lo que realmente nos gustaba, sin importar lo que los demás dijeran que era importante $o$ cualquier otro tipo de presión social. Gracias también a mi tía, mi segunda madre, que siempre ha sido un apoyo insustituible, en muchos sentidos, y en momentos personales muy difíciles durante esta tesis. Gracias a Eva por su admiración. Sin duda, sólo gracias a todos ellos, esta tesis es una realidad.

\section{My dear family}

Nothing a person is or achieves is for exclusive merit; it is a combination of all education received and a pinch of luck. Of the first ingredient, the only ones responsible are my parents. They have always told me that I had the potential to do what I wanted and has encouraged me to do it since I was very young. They convinced the academic director of my first school that I was able to take computer classes when I was 6 , with students ten years older than me (I was also very stubborn). I was finally able to go and do it pretty well. It was my first approach to computing, and since then I've been thinking about ending up doing things like that.

My father is the smartest person I've ever met. He used to spend most of the day reading with an insatiable curiosity. We have more books than any other kind of things at home. Almost every Sunday he took my sister and me to buy books for the Madrid book fair. He transferred to us that passion for knowledge. It has always been (and continues to be) a reference. Mom is a person of great integrity. She always pushed us to do what we really like, no matter what anyone else said was important or any kind of social pressure. Both of them have educated us quite well, making sacrifices for it as well. Thanks to my aunt too, my second mother, who has always been invaluable support, in many senses, in very difficult personal times during this thesis. Thanks to Eva for her admiration. Without any doubt, only thanks to all of them, this thesis is a reality. 


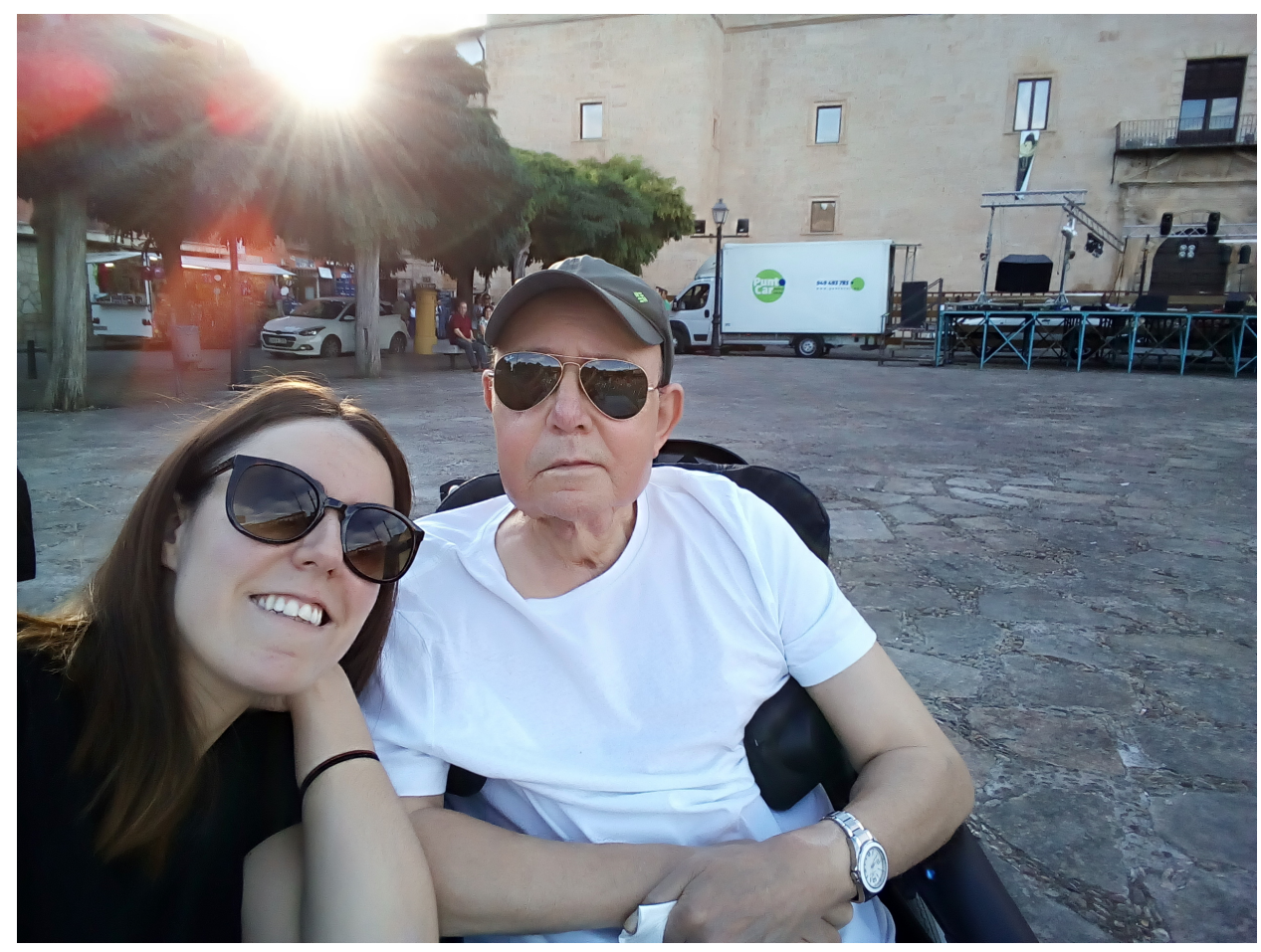





\section{Summary}

Parkinson's disease is the second most common neurodegenerative disorder. It is expected to grow to pandemic proportions by 2040, surpassing Alzheimer's disease. Nonetheless, it remains an idiopathic disease in about $95 \%$ of cases, although it is known that it is caused by the degeneration of dopaminergic neurons of the Substantia Nigra Compacta (SNC). The loss of neurons in this brain area produces a disequilibrium, responsible for the symptoms of the disease, which include tremor of the limbs at rest (the so-called resting tremor (RT)), which is the most characteristic symptom of the disease, muscle rigidity, inability to initiate precise movements (akinesia) and slow motion, especially in complex voluntary movements (bradykinesia).

To alleviate these symptoms, the first option is usually a pharmacological treatment, with or without dopaminergic effects. However, some patients have a tremor with high resistance to medication, even at the highest tolerable doses of levodopa. Besides, the use of levodopa leads to dyskinesias (LID), in which the patient suffers from involuntary movements that may ultimately be worse than the original PD's symptomatology. The second line of treatment in such cases is Deep Brain Stimulation (DBS). DBS consists of the surgical implantation of a neurostimulator, an implantable medical device (IMD) that uses an implanted pulse generator (IPG) to deliver electrical current through a set of electrodes to the surgical target, usually the SubThalamic Nucleus (STN), modulating its functioning.

Despite the growing trend in DBS use, the exact mode of operation and the effects it produces on brain networks remain confused. A higher level of understanding of the neurophysiological changes induced by DBS would be an essential step for at least two main reasons: 1) Firstly, to gain insights into the therapeutic mechanisms of DBS. This would lead to a better understanding of the functioning of the brain under different conditions. For this reason, one of the two objectives of this thesis focuses on studying the effects that stimulation generates at the cortical level. 2) and secondly, the accuracy of DBS could be improved, perhaps avoiding or reducing adverse effects and monitoring treatment response.

The majority work of this thesis focuses on the functioning of the neurostimulator itself. In the context of cardiac illnesses, pacemakers are able to adapt the stimulation to perform event-response in real-time. However, presently, neurostimulators, once implanted, provide continuous stimulation, which may induce adverse effects such as paresthesia, psychiatric or cognitive malfunction and even an increased risk of suicide. Real-time adaptive (closed-loop) DBS systems represent a better strategy, in which the device stimulates only when necessary, on-demand thereby 
reducing the adverse effects. These systems will sense continuously a feedback signal, through which to infer a biomarker, that correlates with the patient's symptomatology. The implementation of such a strategy in PD requires, therefore, the knowledge of what features of the STN activity change when (or ideally, shortly before) the clinical symptoms appear.

This thesis includes four published works that aim to achieve a closed-loop DBS system with the highest possible level of accuracy. To this end, methods of different areas are explored, from chaos theory to machine learning, achieving $100 \%$ accuracy in the last work presented. 


\section{Resumen}

a enfermedad de Parkinson es el segundo trastorno neurodegenerativo más L común. Se espera que su incidencia crezca hasta alcanzar proporciones pandémicas en 2040, superando a la enfermedad de Alzheimer. Sin embargo, sigue siendo una enfermedad idiopática en aproximadamente el $95 \%$ de los casos, aunque se sabe que es causada por la degeneración de las neuronas dopaminérgicas de la Substancia Nigra Compacta (SNC). La pérdida de neuronas en este área cerebral produce un desequilibrio, responsable de los síntomas de la enfermedad, que incluyen el temblor de las extremidades estando el paciente en reposo (el llamado temblor en reposo (RT, de sus siglas en inglés)), que es el síntoma más característico de la enfermedad, la rigidez muscular, la incapacidad para iniciar movimientos precisos (akinesia) y una lentitud de movimiento, especialmente en movimientos voluntarios complejos (bradicinesia).

Para aliviar estos síntomas, la primera opción suele ser un tratamiento farmacológico, con o sin efectos dopaminérgicos. Sin embargo, algunos pacientes presentan un temblor con alta resistencia a la medicación, incluso a las dosis tolerables más altas de levodopa, el farmaco más común. Además, el uso de levodopa induce discinesias (LID, siglas en inglés), en las que el paciente sufre de movimientos involuntarios que pueden ser peores que la sintomatología original. La segunda línea de tratamiento en estos casos es la Estimulación Cerebral Profunda (DBS, siglas en inglés). La DBS consiste en la implantación quirúrgica de un neuroestimulador, un dispositivo médico implantable (IMD, siglas en inglés) que utiliza un generador de pulso implantado (IPG, siglas en inglés) para proveer corriente eléctrica a través de un conjunto de electrodos en la diana quirúrgica, generalmente el Núcleo Subtalámico (STN, siglas en inglés), modulando su funcionamiento.

A pesar de la tendencia creciente en el uso de DBS, el modo exacto de operación y los efectos que produce en las redes cerebrales siguen siendo un misterio. Un mayor nivel de comprensión de los cambios neurofisiológicos inducidos por la DBS sería un paso esencial por, al menos, dos razones principales: 1) En primer lugar, obtener información sobre los mecanismos terapéuticos de la DBS. Esto llevaría a una mejor comprensión del funcionamiento del cerebro en diferentes condiciones. Por esta razón, uno de los dos objetivos de esta tesis se centra en el estudio de los efectos que la estimulación genera a nivel cortical. 2) y en segundo lugar, el nivel de precisión de la DBS podría mejorarse, tal vez evitando o reduciendo los efectos adversos y monitoreando la respuesta al tratamiento.

La mayor parte del trabajo de esta tesis se centra en el funcionamiento del propio neuroestimulador. En el contexto de las enfermedades cardíacas, los marcapasos 
son capaces de adaptar la estimulación para proporcionar respuesta a eventos en tiempo real. Sin embargo, actualmente, los neuroestimuladores, una vez implantados, proporcionan estimulación continua, que puede inducir efectos adversos como parestesia, disfuncines psiquiátrica o cognitiva e incluso un mayor riesgo de suicidio. Además, el tratamiento actual requiere que la batería se cambie en promedio cada 3-5 años, aunque en la práctica, ocurre con más frecuencia para evitar el deterioro del tratamiento.

Los sistemas DBS de ciclo cerrado (closed-loop) representan una mejor estrategia, en la que el dispositivo estimula sólo cuando es necesario, bajo demanda, reduciendo así los efectos adversos. Estos sistemas sensearán continuamente una señal de retroalimentación, a través de la cual inferir un biomarcador, que se correlacionará con la sintomatología del paciente. La implementación de tal estrategia requiere, por lo tanto, el conocimiento de qué características de la actividad del área subtalámica cambian cuando (o idealmente, un poco antes) aparecen los síntomas clínicos.

Esta tesis incluye cuatro trabajos publicados cuyo objetivo es lograr un sistema closed-loop DBS con el mayor nivel de precisión posible. Para ello, se exploran métodos de diferentes áreas, desde teoría del caos hasta diferentes métodos de machine learning, logrando una precisión del $100 \%$ en el último trabajo presentado. 


\section{Acronyms}

$\begin{array}{lll}\text { PD } & - & \text { Parkinson's Disease } \\ \text { RT } & - & \text { Resting Tremor } \\ \text { NT } & - & \text { Atremorous State } \\ \text { TO } & - & \text { Tremor Onset } \\ \text { T } & - & \text { Tremor } \\ \text { DBS } & - & \text { Deep brain stimulation } \\ \text { LFP } & - & \text { Local field potential } \\ \text { MEG } & - & \text { Magnetoencephalography } \\ \text { EMG } & - & \text { Electromyography } \\ \text { STN } & - & \text { Sub Thalamic Nucleus } \\ \text { BG } & - & \text { Basal Ganglia } \\ \text { CL-DBS } & - & \text { Closed-loop Deep Brain Stimulation } \\ \text { FC } & - & \text { Functional Connectivity } \\ \text { EC } & - & \text { Effective Connectivity } \\ \epsilon-\text { RN } & - & \text { Recurrence Networks } \\ \text { AI } & - & \text { Artificial Intelligence } \\ \text { ML } & - & \text { Machine Learning } \\ \text { ANN } & - & \text { Artificial Neural Network } \\ \text { DSM } & - & \text { Data Stream Mining } \\ \text { CBPT } & - & \text { Cluster based permutation test }\end{array}$





\section{$\mathbf{T}$ \\ Introduction}

I believe that only scientists can understand the universe. It is not so much I have confidence in scientists being right, but that I have so much in non-scientists being wrong. Isaac Asimov

It is necessary to energetically shake the forest of dormant cerebral neurons; it is necessary to make

them vibrate with the emotion of the new and to infuse them with noble and elevated restlessness.

Santiago Ramón y Cajal 



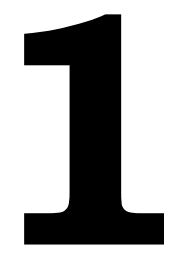

\section{Introduction to Parkinson's Disease}

The movement is defined as the act of moving, that is, to make a body or a part of it change its physical location. This is a simple concept, but at a physiological level, it is much more complex.

This chapter explains how the movement is formed, the structures involved in it, and how it is altered in movement disorders. 
This thesis begins with a brief review of basic concepts about the brain, describing the minimum unit of functioning, the neuron, and the mechanisms they use to communicate and perform complex functions. Besides, because the topic of application of this thesis is movement disorders, we will see the functioning of the basal ganglia. It will be explained in detail how the different nuclei interact with each other, forming a closed circuit, responsible for refining the signal produced in the motor cortex. It will also be explained how the lack of dopamine alters this complex circuit, triggering a cascading effect on the nuclei involved. As a consequence, they will be incorrectly stimulated and inhibited, resulting in movement disorders.

This chapter, like the thesis as a whole, is focused on Parkinson's disease. The main features of this disease will be introduced here, the symptomatology it produces, its prevalence and the primary treatments to alleviate it. Particular emphasis will be placed on explaining the surgical treatment based on deep brain stimulation, being the objective of this thesis, the improvement of this procedure.

This chapter contains information of general knowledge, from the functioning of the basal ganglia to the description of Parkinson's disease, its symptoms and treatments. This information has been studied and summarised from [1], a medical reference book specialising in Parkinson's disease and movement disorders (In order not to repeat a large number of times this reference, it is only cited here).

\subsection{Brief introduction to the brain}

\section{Brain structure}

The human brain has been described as the most complex system in the known universe [2], and not without reason; it possesses an astonishing complexity. It is composed of a network of glia, neurons and nerve pathways and is the most important part of the Central Nervous System. The part of the brain that occupies most of the surface is the telencephalon. Its components are the cerebral cortex, basal ganglia and limbic system.

Cerebral cortex . The cerebral cortex is the outermost sheet of the human brain. It covers above the rest of the brain, and is the area in which the information necessary to carry out the most complex mental processes is integrated, since the information that reaches this region has already been partially processed by other brain structures.

The cortex is divided into two cerebral hemispheres that are almost symmetrical to the naked eye, although, on a microscopic scale, they are very different. Each hemisphere is composed of four lobes: parietal, frontal, occipital, temporal, and insula. Each of these lobes is involved in different processes. The cortex is mostly made up of pyramidal neurons. They are organized in a structured way with apical dendrites in parallel to each other and perpendicular to the cortex surface, forming cortical columns. They were discovered by the Spanish neuroscientist Santiago Ramón y Cajal. His drawings on these neurons marked a milestone in neuroscience, 
for which he won the Nobel Prize. One of his drawings can be seen in figure 1.1 .

Basal ganglia (BG) . The second component of the telencephalon is the set formed by the basal ganglia. These are a group of structures located below the cerebral cortex and symmetrically distributed under each of the hemispheres. The basal ganglia are the parts of the brain that allow complex and precise movements. These structures play an important role in Parkinson's disease. Therefore, the circuit that operates with these nodes and their operation mode will be described at a low level in the next section.

At a cellular level, the brain is mainly constituted of glial cells and neurons. Glial cells are part of a support system and are essential for the proper functioning of the nervous system tissue. Unlike neurons, glial cells have no axons, dendrite or nerve pathways. For their part, the primary function of neurons is to receive, process and transmit information through chemical and electrical signals.

\section{Neurons}

The neuron is the most basic structural and functional element of the nervous system. They are in charge of transmitting information, forming networks that specialize in neuronal functions. For this purpose, neurons integrate information from other neurons through neurotransmitters (electrical or chemical). The information is integrated and transmitted along the axon so that it reaches the next neuron. The figure 1.2 shows a typical neuron.

Like other cells in the body, the neuron consists of a cell body and a nucleus, called soma. However, it also consists of an axon and ramifications of input, called dendrites, and output, called axon terminals. Through the dendrites, the cell receives information from neighbouring neurons, in the form of electrochemical impulses. This information is processed in the soma. If the input information exceeds a threshold value, a potential action is unleashed, and the information is propagated through the axon, reaching the terminals.

The action potential in a neuron is triggered either because it receives a considerable impulse from the previous neuron, or because the sum of the inputs exceeds the threshold value. It is thus a sum of local potentials. The action potential is a fast (around 1-2 ms) electrical impulse that transmits the nerve impulse from one neuron to the next, via a synapse. The action potential starts at the beginning of the axon; an area called axon hillock, which is an area rich in sodium channels regulated by voltage and spreads along the axon, ending in the terminals. There is a space between the terminals of a neuron and the dendrite of the followings, called synaptic area. It is here where the neurotransmitters, released during the synapse, accumulate. Depending on the existing neurotransmitters in this area, the signal will be excitatory or inhibitory, as explained in more detail later.

Once generated, the action potential is always the same, of the same magnitude, 


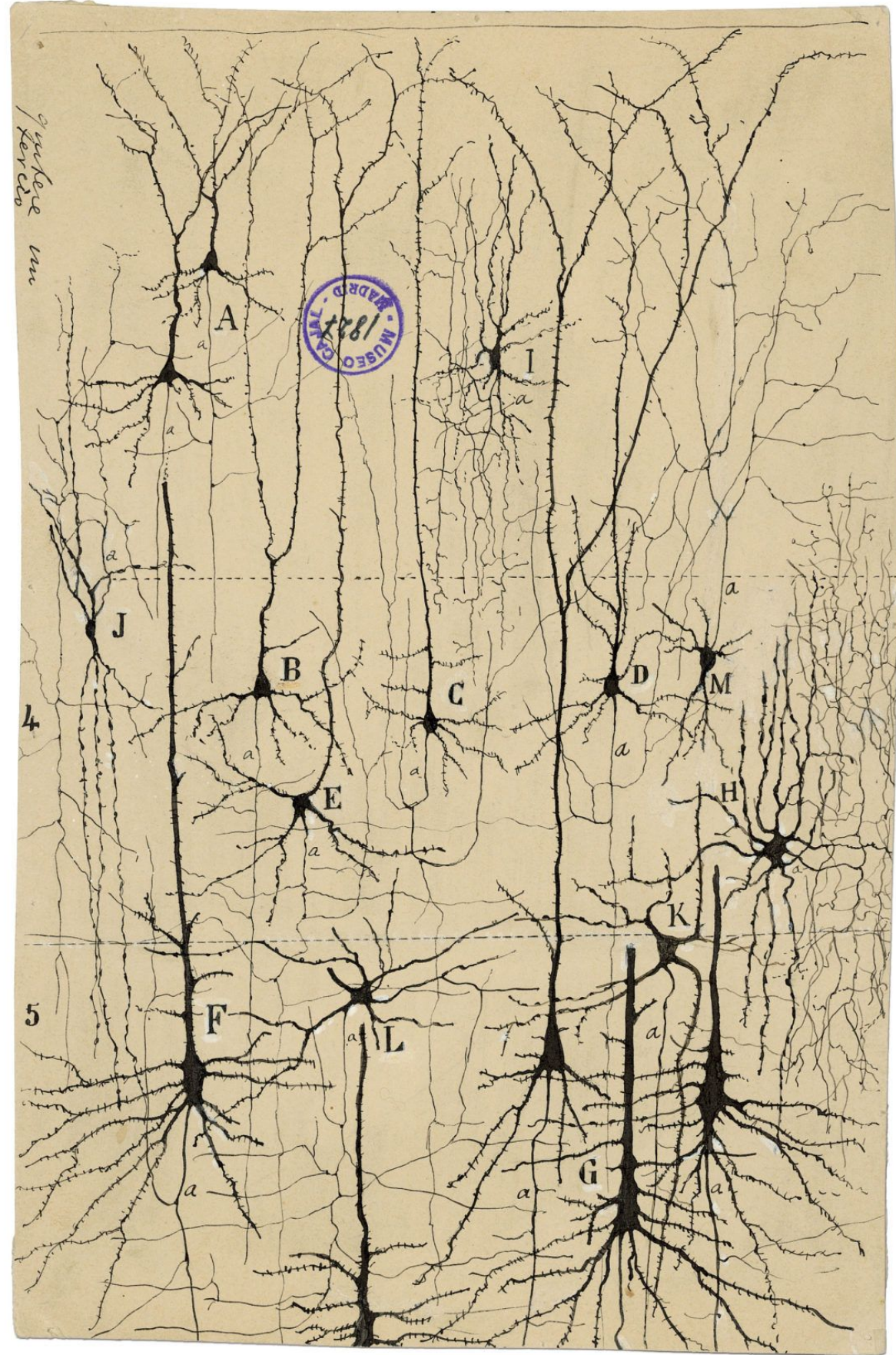

Figure 1.1: Original pyramidal neurons drawing by Santiago Ramón y Cajal. Cajal Museum 1827, Madrid. 


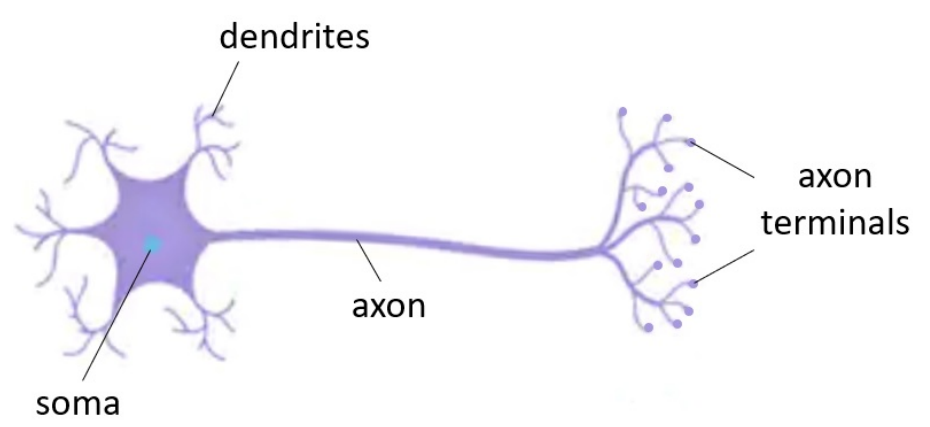

Figure 1.2: Basic neuron's components. From left to right: dendrites, which collect other neuron impulses; the cell body, where the nucleus (soma) is allocated; the axon, the channel that sends the information to the postsynaptic cell; and finally the synapse, the union between the two cells needed for the communication.

around $35 \mathrm{mV}$, not depending on the magnitude of the input that has generated it. Therefore it is said that action potentials follow the "all or nothing" law. In addition, since the membrane maintains the voltage, the action potential maintains its intensity along the entire path from the axon hillock to the terminals.

When the cell is at rest, the membrane is around $-90 \mathrm{mV}$, having a negative polarity inside. Intra-membrane, there is a high concentration of $K+$, and outside the membrane, there is a high concentration of $\mathrm{Na}$. These ions will move through the $\mathrm{Na}-\mathrm{K}$ channels in favour of the gradient, i.e. passing from where there is more to where there is less concentration, so the $K+$ ions will come out, and the $\mathrm{Na}+$ ions will come in. As stated above, the action potential is triggered when the membrane reaches a threshold in response to incoming local potential from other neurons, following three stages:

1. Depolarization: The incoming signals generate an electric current in the membrane. This causes the voltage-regulated sodium channels to open, and $\mathrm{Na}+$ moves into the axon, producing a change in the polarity between outside and inside of the neuron.

2. Repolarization: Voltage-regulated potassium channels open, causing $K+$ ions to move out of the axon. Voltage-regulated sodium channels close.

3. Undershoot: Potassium channels are slower to close than sodium channels. Therefore, at the end of the repolarization stage, a small hyperpolarization takes place, which causes the neuron to enter into a refractory period during which it cannot be excited. When the potassium channels are closed, the concentration of $\mathrm{K}+$ and $\mathrm{Na}+$ ions is restored, reestablishing the resting potential.

When the action potential reaches the axon terminals, it triggers the release of neurotransmitters. If the neurotransmitter is excitatory, such as the amino acid 
glutamate, an excitatory postsynaptic potential (EPSP) is activated. Positive ions flow inwards the postsynaptic neuron, therefore depolarizing the cell membrane. Conversely, when the neurotransmitter is inhibitory, such as the $\gamma$-aminobutyric acid (GABA), an inhibitory postsynaptic potential (IPSP) is triggered. Either negative ions flow inwards, or positive ions flows outwards the postsynaptic neuron, therefore polarizing the cell membrane.

\subsection{Functioning of the basal ganglia}

oluntary motor activity, such as lifting an arm to reach an object, is the result of a series of movements that must be perfectly coordinated. The intention to carry out the movement gives rise to the voluntary movement. This action originates in the cerebral cortex. If it is a previously learned movement, such as walking, the impulse/signal will be transmitted from the premotor cortex, related to a memory bank of movements. Otherwise, it will be transmitted from the motor cortex. We will call the node where the action begins, origin node from here on. From the origin node, the impulse is transmitted to the spinal cord by two pathways, the pyramidal pathway and the extrapyramidal pathway. Most movements require the coordinated action of both systems.

The pyramidal system is formed by axons, whose neuronal nuclei are found in the motor cortex of the frontal lobe, and that in their trajectory to the spinal cord are crossed at the level of the spinal bulb, the so-called decussation of the pyramids. Through the pyramidal pathway, the motor cortex sends impulses to the spinal cord, where they contact the second motor neuron, located in the anterior horn of the medulla. The axons of this second neuron send the information directly to the muscles. In theory with a circuit of only two neurons, it would be possible to raise the arm and takes an object. However, the resulting movement would not be too precise. Probably we would throw a few objects trying to reach the desired one.

It is the basal ganglia (BG), a set of subcortical nuclei, that refine the movement initiated in the motor cortex. They are located in the medial portion of the cerebral hemispheres and the cerebral trunk. The most relevant nuclei of the basal ganglia are the Striatum, the Globus Pallidus, the Substantia nigra compacta and the Subthalamic nucleus. Its location in the brain can be seen in figure 1.3.

The Striatum is made up of two distinct structures: the Caudate nucleus and the Putamen, dorsal and ventral structures respectively.

The globus pallidus (GP) is located medial to the Putamen, from which the external medullary lamina separates it and is divided into two portions by the internal medullary lamina, the external and the internal Globus Pallidus, which assume different functions. Both have incoming connections from the Striatum. The striatal neurons projecting into the internal globus pallidus have a high expression of messenger RNA for D1 dopamine receptors. While those that project to the external globus pallidus have a higher expression of messenger RNA for $D 2$ receptors. 


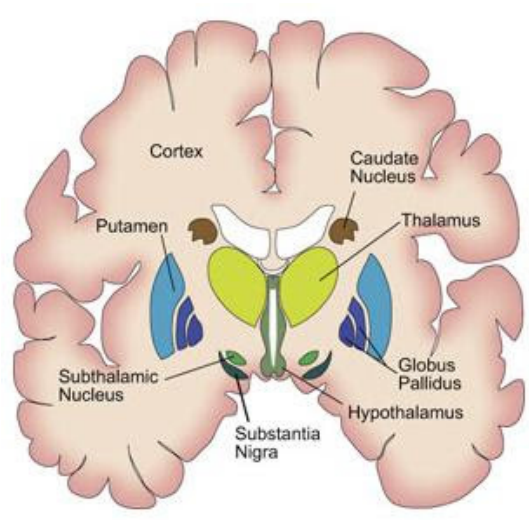

Figure 1.3: Basal Ganglia Nuclei

The Substantia nigra compacta (SNc), so-called because of the colour of the cells that make it up, loaded with melanin. It is located in the dorsal mesencephalon and is divided into Pars compacta and Pars reticulata, the latter similar in function to the internal Globus Pallidus.

Subthalamic Nucleus (STN), located in the subthalamic region. It is the major part of the subthalamus, and it is located ventral to the Thalamus and dorsal to the substantia nigra. It is composed mainly of neurons of quite long and little sparsely dendrites.

All these nuclei interact for the correct functioning of the basal ganglia. The Thalamus acts as a switch, sending to the motor cortex the resulting output of the circuit forming the set of basal ganglia.

\section{Extra-pyramidal pathway}

The motor cortex, while sending impulses through the pyramidal pathway, also sends them to the BG, more specifically to the Striatum, through the extrapyramidal pathway. This signal runs through the basal ganglia, where it is regulated and nuanced, thus returning modulated to the motor cortex. As a result, the signal that reaches the medulla is more precise and perfect, and so is the movement performed. From an engineering perspective, it could be said that the basal ganglia work like applying filters to the initial signal that makes the final output more precise, better defined.

\section{But, how exactly is this information exchanged at a low level?}

Before entering the detailed description of the circuit, it is necessary to know the default function of the nodes involved: The STN and the thalamus always stimulate, while the basal function of the striatum, GPe and GPi are always to inhibit. These 
basal functions will be exacerbated or diminished depending on the activity coming from the previous nucleus (the input signal).

In subjects absent from disease, SNc releases dopamine (2- (3,4-dihydroxyphenyl) ethylamine), stimulating the striatum. Two primary pathways emerge from the striatum, the so-called direct and indirect pathways. They differ in their connections and neurochemical characteristics. Both operate together in equilibrium as depicted in figure 1.4 and as explained here:

\section{Direct pathway}

Activation of the direct pathway results in facilitation of movement. In the presence of dopamine, released by the SNc, this pathway is activated in the following way: The cortex stimulates the striatum. The striatum, when stimulated, increase its activity. With high activity, and through D1 receptor, it will inhibit the GPi. Inhibited GPi will decrease its activity. By decreasing its activity, it will inhibit a little bit the thalamus. The thalamus is therefore little inhibited, and in consequence, it will have high activity, thus being able to stimulate the cortex, favouring movement.

\section{Indirect pathway}

Contrary to the direct pathway, the activation of the indirect pathway results in an inhibition of movement. In the presence of dopamine projected from the SNc to the striatum, the circuit operates as follows:

As in the direct pathway, the indirect pathway circuit commences when the cortex stimulates the striatum. As before, since it is being stimulated, the striatum is going to increase its activity. Having a high activity and, through D2 receptor, the inhibition to GPe will be low. Inhibiting GPe little will cause its activity to increase. By increasing its activity, it will significantly inhibit the STN. The STN, being acutely inhibited, will decrease its activity. By decreasing its activity, it will stimulate little the GPi.

Similarly to the direct pathway, a little stimulated GPi will lower its activity, inhibiting in turn little to the thalamus. Finally, as in the direct pathway, the thalamus, which is little inhibited, increases its activity, stimulating the cortex and promoting movement.

\subsection{Pathophysiology of Parkinson's disease}

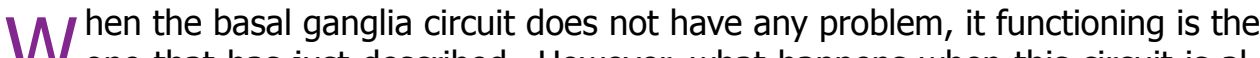
one that has just described. However, what happens when this circuit is altered? What are the outcomes? As a result of the inadequate functioning of the circuits of the basal ganglia, there are a series of disorders that can produce hyper and hypokinetic syndromes, depending on whether they produce excess or defect of movement, respectively. 


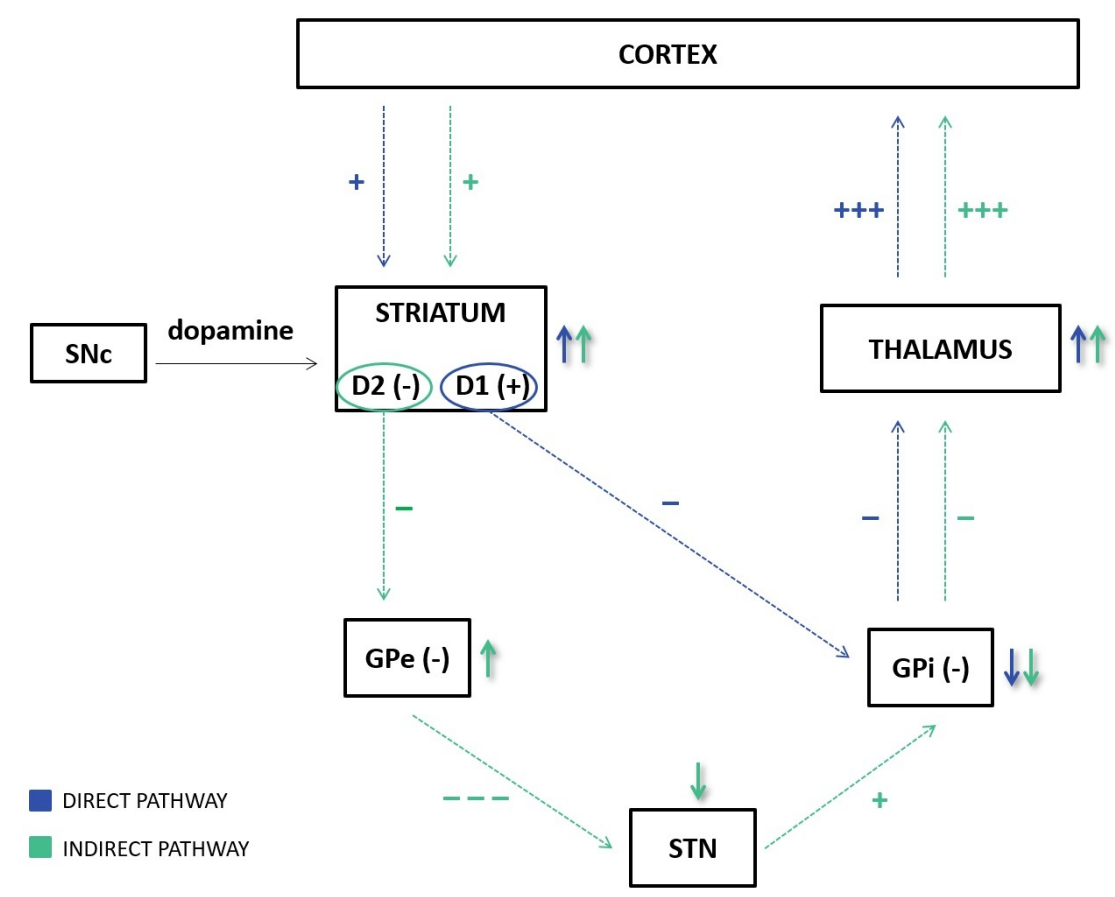

Figure 1.4: Basal ganglia circuit in their normal mode of operation (non-pathological subjects) 


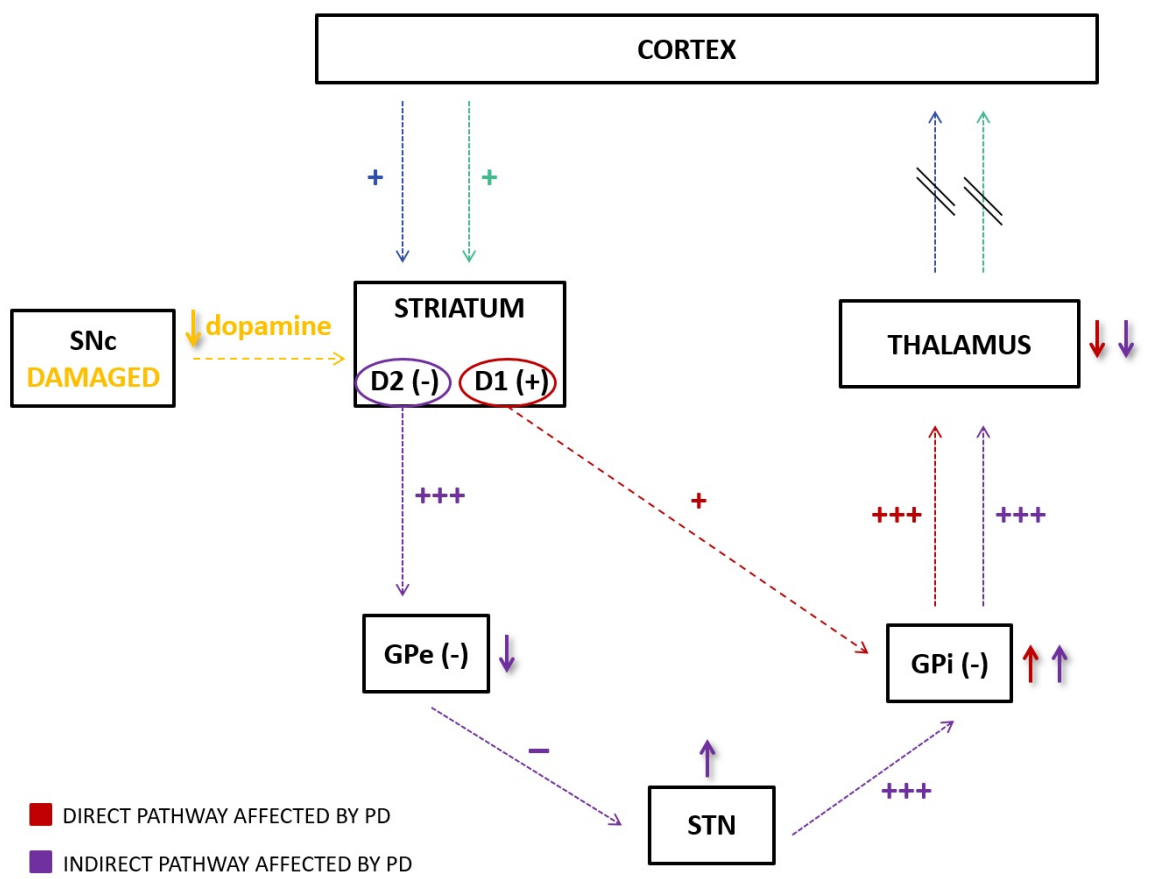

Figure 1.5: Basal ganglia circuit with its functioning affected by Parkinson's 


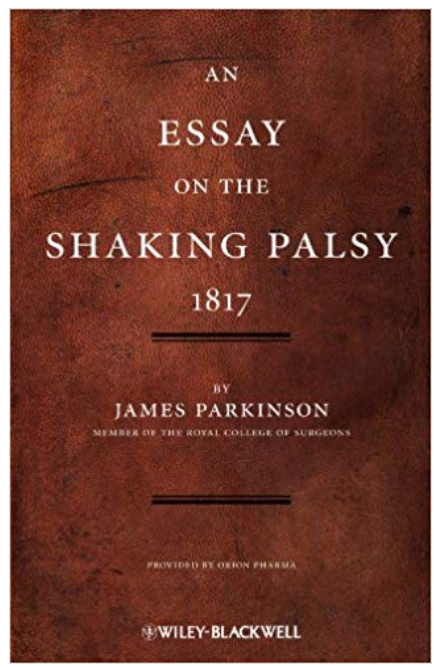

Parkinson's is the most representative of hypokinetic syndromes. It is the second most common neurodegenerative disorder. It was first described in 1817 by James Parkinson in his work Essay on shaking palsy [3], where he defined the disease as a trembling paralysis. Although it was not until the 1960s when it was discovered that it was caused by the death of dopaminergic neurons in the SNc. This loss of neurons produces an imbalance between the direct and the indirect pathways, with a fundamental predominance of the indirect one. This imbalance is responsible for the noticeable symptoms of the disease.

\section{What imbalances are caused by the death of dopaminergic neurons?}

When the neurons of SNc start dying, the release of dopamine begins to decrease. In the absence of dopamine D1 receptor, which is stimulated in the presence of dopamine, start being not stimulated. This triggers a series of cascading effects: In the direct pathway, The GPi is not going to be inhibited by the striatum, so its activity will be higher. By having a higher activity, GPi will significantly inhibit the thalamus. For its part, if the thalamus is very inhibited, it will decrease its activity, so it will not be able to stimulate the cortex, thus preventing movement.

In a similar way, the indirect pathway will be affected. In the absence of dopamine, the $\mathrm{D} 2$ receptor will not be inhibited. In this way, the striatum will inhibit a lot the $\mathrm{GPe}$, which decreases its activity, inhibiting in turn little the STN. The little inhibited STN increases its activity, stimulating the GPi a lot. As in the direct pathway, a highly stimulated GPi increases its activity, inhibiting a lot the thalamus, which in turn is not able to stimulate the cortex, preventing movement.

As a result of an inhibited cortex, the movements are more clumsy and inaccurate, thus producing the main symptoms of Parkinson's disease. This model is shown in figure 1.5. In summary, in the absence of dopamine, the direct pathway will be diminished while the indirect pathway will be exacerbated, thus preventing both the movement.

\section{Pathogenesis}

Several neuroimaging studies show a reduction of dopaminergic neurons by up to $80 \%$. Figure 1.6 shows a PET image of a normal subject versus a subject affected with PD [4]. The pathogenesis is, therefore focused on trying to understand the mechanisms that lead to neuronal death. There are several hypotheses in this regard, here we summarise the main ones: 


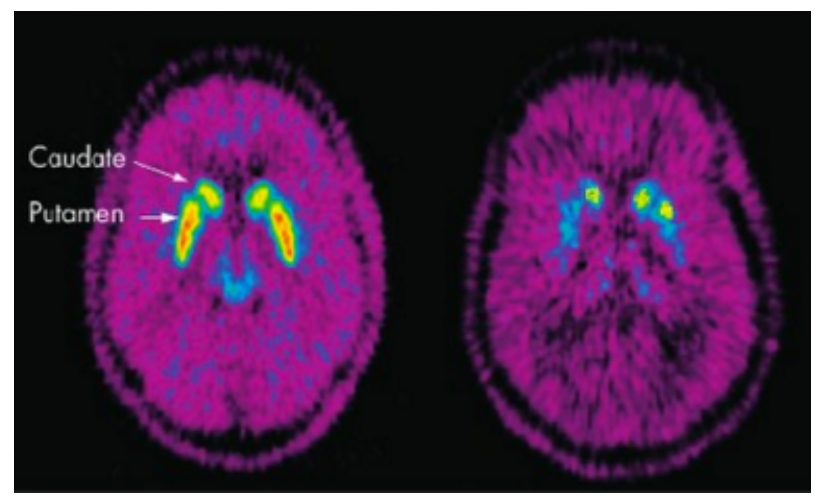

Figure 1.6: 18 F-Dopa PET of a healthy subject (left) and a PD patient (right)

Free radicals . Free radicals are unstable molecules that occur naturally due to different chemical reactions within the body. These molecules lack an electron, which they try to recover by reacting with other molecules in oxidation processes, usually with metals, such as iron. These processes can damage and cause the death of neurons. This theory is supported by the discovery of elevated levels of iron in the SNc, perhaps as a result of a deficiency of ferritin, which would isolate iron and prevent its oxidation.

Toxins . This hypothesis suggests that the death of dopaminergic neurons may be due to the action of certain toxins such as MPTP (1-methyl-4-phenyl-1,2,3,6, -tetrahydropyridine). Despite being only a hypothesis, some foundations like that of Michael J. Fox contribute actively in its investigation today.

Genetic factors . One of the most recent hypothesis holds that death can be caused by genetic factors. This hypothesis is based on the fact that between 15 and $25 \%$ of patients with Parkinson's have had a family member who suffered from the disease.

Neuronal deterioration . This last hypothesis holds that the deterioration of dopaminergic neurons is normal due to age. What happens in patients with Parkinson's is that, due to some unknown cause, this process accelerates.

The real origin is still to be discovered, remaining an idiopathic disease in about $95 \%$ of cases [5].

\section{Symptomatology}

The imbalance between the direct and indirect pathways constitutes the origin of the symptoms of the disease, which include tremor of the limbs at rest (the socalled resting tremor $(\mathrm{RT})$ ), muscle rigidity, inability to initiate precise movements 
(akinesia) and slow motion, especially in complex voluntary movements (bradykinesia).

Resting tremor . It is the most common and characteristic symptom of this condition. It is a regular and rhythmic movement, which oscillates in the frequency band of [3.5 - 7] Hz, which becomes more evident in the resting state of the subject, diminishing or disappearing with the beginning of the movement. It usually begins in one hand, then extends to the remaining limbs, and sometimes to the muscles of the face and neck.

Rigidity . Consists of an increase in resistance to stretching of the extremities, as a consequence of the simultaneous activation of the flexor and extensor muscles. This situation can occur continuously or at intervals. Due to this rigidity, the characteristic posture of parkinsonism is a flexion, both of the head and trunk and the joints of arms and legs.

Akinesia and Bradykinesia . Akinesia is the inability to initiate precise movements. For its part, bradykinesia is the reduction or slowdown of voluntary automatic motor acts. Both symptoms incapacitate the patient to perform movements that were previously almost automatic.

Alteration of postural reflexes. This symptom refers to the difficulty in correcting abnormalities in posture. Thus, when moving, the anticipation of the loss of the continuous equilibrium that the motion entails is lost. For this reason, it is typical for PD patients to perform a series of quick and small steps to move forward, to avoid falling, the so-called propulsive march.

Apart from the symptoms described here, there are another series of symptoms associated with the disease, which, although they do not affect all patients, cause serious disorders, such as difficulty in swallowing, speech alteration and lack of facial expression.

\section{Prevalence}

Prevalence is the proportion of individuals in a population that suffer from the disease at a given time or period. Its calculation is estimated as:

$$
\text { prevalence }=\frac{\text { total population }}{\text { number of subject with the disease }}
$$

The prevalence of PD in Europe in women is 1.267, and 1.535 in men [6]. Parkinson's disease is nowadays the second most prevalent neurodegenerative conditions, just behind Alzheimer Disease [7]. However, new research shows that the number of people with Parkinson's disease will soon grow to pandemic proportions [8]. The authors point out that between 1990 and 2015, the prevalence of Parkinson's 
more than doubled, and it is estimated that by 2040 , the number of people with Parkinson's will be around to 14.2 million, surpassing Alzheimer disease.

\subsection{Treatments}

espite its prevalence, currently, there is no treatment to cure the disease, focusing the different existing solutions in maintaining the functioning of the basal ganglia as normal as possible. The treatments can be divided into two sets: pharmacological and surgical.

\section{Pharmacological treatments}

Pharmacological treatments are divided into those that have a dopaminergic effect and those that do not. Among the dopaminergic drugs, we find levodopa and the dopamine agonists. Currently, levodopa is the primary pharmacological treatment for PD. It is the main precursor of dopamine in the brain. It is used by the neurons for the synthesis of dopamine and thus supplying the decreasing supply of dopamine in the brain. The pharmacological treatments are based on levodopa instead of dopamine because the latter is not able to cross the blood-brain barrier. For its part, dopamine agonists are treatments aimed at stimulating dopamine receptors, thus "mimicking" the action of dopamine in the brain. They can be used alone or in conjunction with levodopa.

Finally, there are a set of drugs without dopaminergic effect. Their effect is not due to a primary dopaminergic effect, but an effect at other levels of neurotransmission, such as COMT inhibitors, drugs able to inhibit the action of catechol-O-methyl transferase, an enzyme involved in degrading neurotransmitters. Thus, this treatment is aimed to inhibit levodopa degradation.

Throughout the course of the disease, after a period of treatment with levodopa or dopamine agonists, the response of the motor symptoms to the medication changes. As a consequence of prolonged pharmacological treatment, especially in the case of levodopa, a series of motor complications can appear, including motor fluctuations, dyskinesias, and the so-called ON-OFF effect.

Levodopa-induced dyskinesia (LID) makes the patient suffers from involuntary movements. For its hand, ON-OFF effect makes the patient experience ON periods in which the drug works, being alternated with OFF periods in which the drug has little or no effect, and the patient continues suffering the PD symptoms.

These motor complications can limit the patient's life, even to a greater extent than the original symptoms, to the point of making it incapacitate. These complications are due to two fundamental circumstances:

- The progressive degeneration of the substantia nigra entails the denervation of the nigrostriatal dopaminergic system.

- Chronic exposure to dopaminergic agents that act on striatal dopaminergic receptors. The activation of the dopaminergic receptors within the basal ganglia is pulsatile, coinciding with the administration of the drug, as opposed 
to the constant activation that occurs in a normal situation - without pathology. The longer the half-life of the drug, the more continuous the release of dopamine, and therefore, the less risk of motor complications there will be.

\section{Surgical treatments}

Surgical treatment is the next option in those cases in which the pharmacological treatment is no longer indicated. This happens mainly in patients with LID or with advanced stages of the disease that do not respond well to pharmacological treatment.

The nuclei on which surgery is performed are called surgical targets. In PD, the target nuclei for surgical treatment is usually the STN, since acting on it, the best results are obtained, attenuating its hyperactivity caused by the disease. The main surgical techniques are:

The lesion of target nuclei . The objective of this technique is to cancel the function of any of the BG nuclei, producing a lesion. In this way, the contribution of this nucleus in the BG circuit is cancelled.

The intervention is usually performed by inserting an electrode to the target nuclei, on which an electric current is discharged, burning the selected area. A novel way to carry out this injury is through the use of MR-guided focused ultrasound (MRgFUS), a non-invasive technique able to perform ablation of the soft tissue of the target nuclei [9].

Deep Brain Stimulation . The function of the target nuclei can also be annulled employing the continuous stimulation of said nuclei with high frequencies, which causes reversible inhibition of their neuronal activity. This technique is known as deep brain stimulation (DBS). It supposes an alternative treatment to surgical ablation. Since this procedure is the central core of this thesis, it will be explained in more detail in the following section.

Dopamine cell transplantation . It consists of transplanting the dead dopaminergic cells. Adult cells that produce dopamine or stem cells from embryos or adults can be implanted [10]. These techniques are in the process of experimentation, not being a therapeutic alternative that is carried out in the clinic.

\subsection{Deep brain stimulation}

$\mathrm{D}$ eep brain stimulation consists of the surgical implantation of a medical device (IMD), called neurostimulator, that uses an implanted pulse generator (IPG) to deliver electrical current through a set of electrodes to precisely targeted areas in the brain, modulating its pathological functioning. This device is sometimes referred to as the brain pacemaker, although, as we will see below, they do not work the same as cardiac pacemakers. 
The main usage application of DBS is in movement disorders. It was approved by the Food and Drug Administration (FDA) as a treatment for PD in 2002 [11], and since then it has become a necessary treatment, especially in cases where the patient has resistance to oral treatment. Usually, DBS is administered in conjunction with levodopa, if necessary. DBS is also a treatment widely used in other movement disorders such as Essential tremor and Dystonia. However, no good results have been obtained in its use for Action tremor, present in patients suffering from stroke, multiple sclerosis or trauma [12].

Given the positive outcomes obtained by DBS in movement disorders, it has been applied to other diseases such as epilepsy and depression [13-15]. Also, consideration is currently being given to extending its use to stroke, dementia, pain, vegetative state, addictions, obesity and anorexia $[16,17]$

\section{Target nucleus}

In the case of Parkinson's disease, the target areas are the subthalamic nucleus (STN) or internal globus pallidus (GPi) [18]. STN-DBS is the therapeutic option chosen in most cases, since its stimulation effectively treats the main symptoms that perform well to levodopa [19, 20], including tremor, rigidity, akinesia and postural instability and LID. Moreover, STN-DBS allows a more significant reduction in oral medication [21], and also has a better outcome of the OFF-symptoms [22]. Besides, it requires the parameters to be adjusted more often, which leads to higher battery consumption [23].

\section{Candidate patients}

Not all parkinsonian patients are eligible to be treated with DBS. The decision is based on several variables, being the symptomatology suffered the main one, but it is not the only one:

Symptoms resistant to levodopa are usually also resistant to stimulation. The symptoms that DBS best controls are tremor-dominant PD, LID and levodopa-sensitive OFF symptoms, peak-dose hyperkinesias and biphasic dyskinesias. In summary, the best candidate profile to be treated with DBS is a parkinsonian patient who suffers from intractable tremor, intractable motor fluctuations or adverse effect derived from medication [24].

In addition, there are clear exclusion criteria regarding the other variables: 1) An age over 75 years, 2) chronic immunosuppression, 3) distinct brain atrophy, 4)cognitive deficits, 5)dementia or 6)severe psychiatric disorders [25].

\section{DBS Hardware}

An implantable neurostimulator is composed of an implantable pulse generator, extensions, lead, and electrodes. Moreover, it has external components such as a physician's programmer or patient's programmer. These components are depicted in figure 1.7 [26] and explained hereinbelow. 

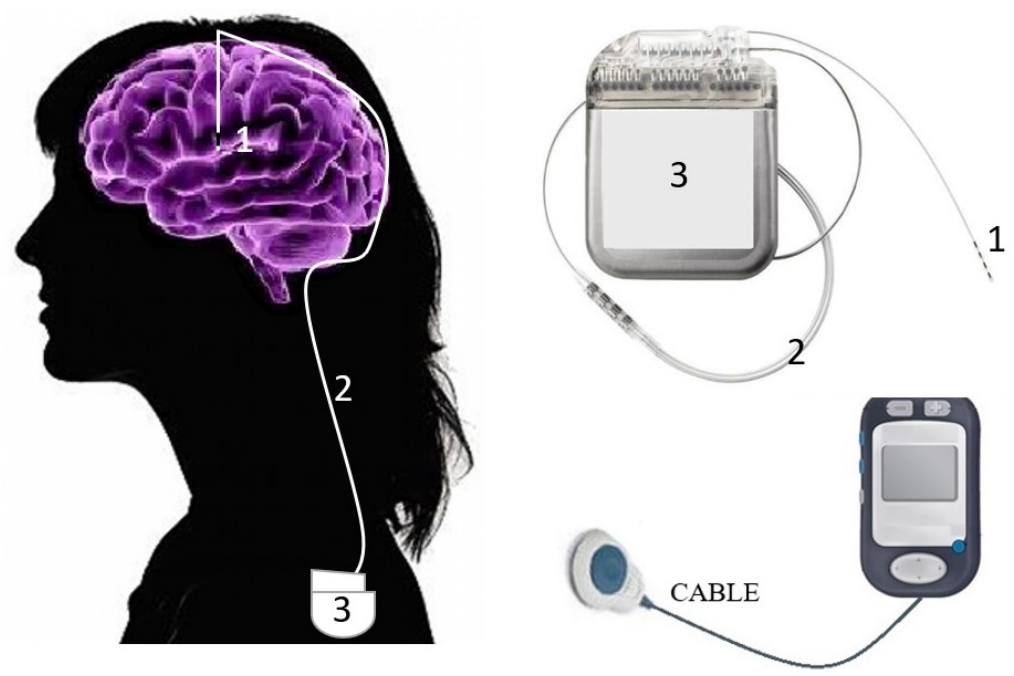

Figure 1.7: Deep Brain Stimulator System Type Medtronic Activa $® P C$

Lead and Electrodes . The led is a wire covered with a protective shell. Its function is to carry the electrical current to the electrodes, that delivers stimulation to the target area. The electrodes are implanted within the target area, while the lead is implanted under the skin of the scalp.

Extension . The extension is a wire with a protective shell that plugs in the IPG with the lead. The connection of these two wires is set up under the scalp, usually behind the ear. There is one extension per lead.

Implantable Pulse Generator . The IPG is the power source of the DBS system. It is usually implanted under the skin in the collarbone area. It is composed of a battery and a chip in charge of delivering the electrical pulses.

Programmer . The function of the programmer is to wirelessly adjust the stimulation parameters of the IPG, as well as turn it off and on. The adjustment of the parameters is carried out by the specialist. Patients can also have a programmer, allowing them to have some control. The programmer is the interface where the specialist specify the desired values for the parameters, and the order to update these values is sent to the IPG.

If the patient has bilateral DBS, he will have implanted the described components, both on the right and left side. 


\section{DBS Software}

An optimal parameter selection process should be carried out in order to maximise the improvement of clinical symptomatology while minimising adverse effects. This process is called "programming". Despite its name, it is a manual process in which the specialist must test combinations of parameters and their respective effect on the patient, in a trial and error process. The programming of these parameters manually requires a great deal of time on the part of the medical staff, since in practice, different combinations are fixed, and the way they work in the patient is tested. This is an iterative and arduous process for both the physician and the patient. Moreover, it is estimated that this process will become more complicated due to the development of new DBS systems and new parameters to be set [27]. The current set of parameters to be adjusted are the optimal electrode contact, and three stimulation parameters: pulse amplitude, pulse width, and frequency.

There are some computational models for the choice of the first parameter. Imaging techniques should be used to check the validation of the electrode location. In this respect, the insertion of the electrodes can be guided during MRI-guided stereotactic neurosurgery. [28]. If this option is not available, an MRI may be performed after surgery, assuming certain precautions.

Concerning the stimulation parameters, at present, there are no systems capable of automatically controlling its value, but are manually set by the specialist during the patient's visits to the hospital. The values determined in the visit remain constant regardless of the fluctuations that the patient may experience.

\section{Future DBS systems}

Programming procedure, despite the help of computer models, is ultimately a subjective evaluation of the clinical improvements during the programming sessions. But not only that, even perfectly determining these parameters during the programming session, does not mean that they perfectly match the patient's condition between visits to the specialist, in fact, this does not happen.

And not only that, these parameters determine the values of the stimulation, but it will be administered continuously. That is, the symptoms that the patient suffers at each moment are not taken into account. As will be seen, this continuous stimulation contributes to the emergence of adverse effects.

Some closed-loop DBS strategies propose an objective and dynamic adjustment of the parameters. However, this is not the final solution since the stimulation would remain continuous. For this reason, there is another branch of techniques aimed at achieving demand-driven stimulation systems. These techniques are explained in more detail in chapter 4. Demand-driven DBS is the main objective of this thesis. In this respect, block 3 presents the studies carried out for this purpose. As will be seen, the accuracy of the proposed systems is improving from the first proposed system to the last one, in which $100 \%$ is achieved using a system using a data stream mining procedure based on the real-time STN connectivity levels. 


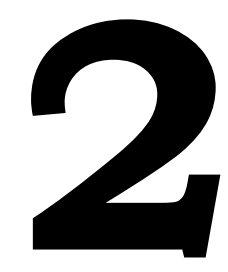

\section{Introduction to Neuroimaging and Analysis Methods}

Since the birth of neuroscience more than a century ago, when Santiago Ramón y Cajal postulated that the nervous system is made up of individual neurons that communicate with each other, considerable progress has been made in the knowledge about the functioning of the brain. However, the brain remains a mystery, and much work is still needed to understand it fully. Indeed, the 21st century has been proclaimed the century of the brain.

Directly observing the structure and functioning of the brain is at the base of modern neuroscience. Neuroimaging techniques have been essential in the last few decades. Advances in this area allow us to observe in real-time the physiological changes that occur in the brain.

This chapter presents the main neuroimaging techniques used in this thesis, with particular emphasis on magnetoencephalography, due to its novelty and complexity. It also introduces all the mathematical methods used in the analysis of neural data used. 


\section{Neural Oscillations}

$\mathrm{N}$

eural activity can be decomposed into different oscillators, also called brain rhythms or brain waves. A brain oscillator is a rhythmic pattern of neural activity, which is repeated periodically around a point of equilibrium. An oscillation is defined by its amplitude, frequency and initial phase, as represented in figure 2.1

Period $(T)$ and Frecuency $(f)$. The period is defined as the time required for a complete oscillation. It is measured in seconds and defined as the inverse of the frequency $T=1 / f$. Related to the period, the frequency is a magnitude that measures the number of oscillations per unit of time. It is measured in hertz $(\mathrm{Hz})$ and can be calculated as $f=1 / T=\omega / 2 \pi$.

Amplitude $(A)$. Represents the maximum distance between the furthest point of an oscillation and the equilibrium point.

Phase $(\phi)$. The phase indicates the instantaneous situation within a cycle, being the fraction of the period elapsed since the initial instant (or to the instant to the state taken as reference). It is measured in radians. Due to radian is a dimensionless unit, radian per second is dimensionally equivalent to Hertz, both are defined as $s^{-1}$.

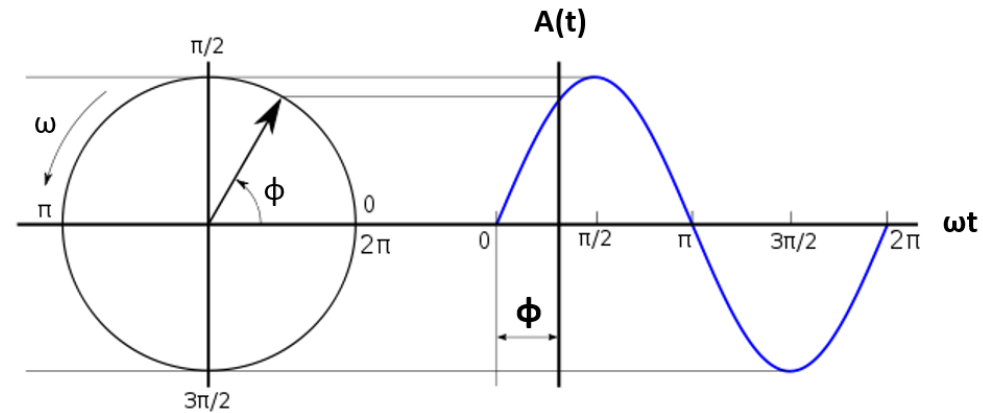

Figure 2.1: Representation of an oscillation of period $T$, amplitude $A$ and initial phase $\phi$

An oscillatory activity can be found in the central nervous system at all levels. At the most elementary level are the spike trains. A spike train is simply a sequence of action potentials generated by a single neuron. Spikes are considered essential in the process of information coding in the brain. When numerous neurons spike in synchrony, they can give rise to oscillations in local field potentials. On a higher level, interaction among neurons originates large scale oscillations, which can generate oscillations at different frequencies than the firing frequency of individual neurons, generating a joint activity at specific frequencies. To give an example, this is the case of the well-known alpha activity. 
The study of neural oscillations reveals oscillatory activity in specific frequency bands. The first frequency band that was studied in electroencephalography was the alpha activity $(\alpha,(8-12 \mathrm{~Hz}))$ that can be detected from the occipital lobe during resting wakefulness state and which increases when the eyes are closed. But there are more defined frequency bands named delta $(\delta,(0.5-4 \mathrm{~Hz}))$, theta $(\theta,(4-8 \mathrm{~Hz}))$, beta $(\beta,(12-30 \mathrm{~Hz}))$ and gamma $(\gamma,(30-100 \mathrm{~Hz}))$ bands, represented in figure 2.2 .

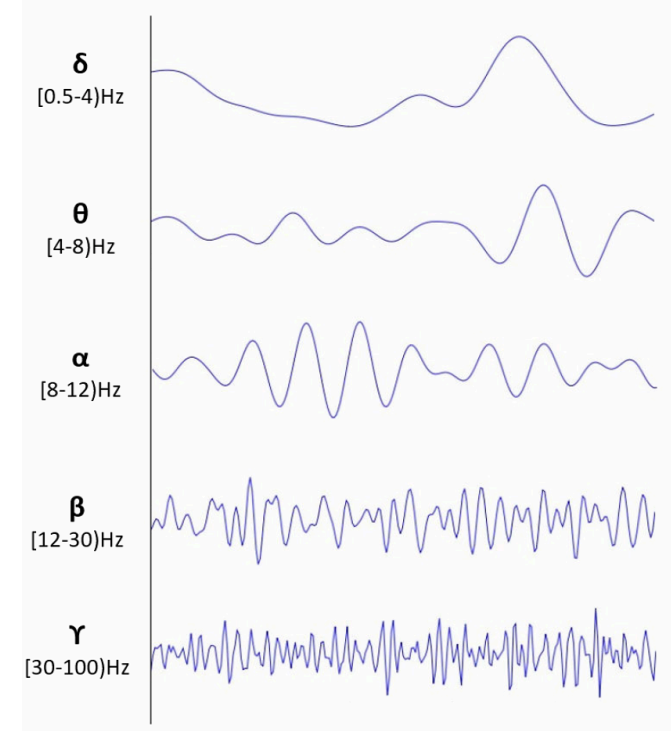

Figure 2.2: Representation of the classical electrophysiology frequency bands

The oscillatory activity at medium and large scale, which gives rise to activity in frequency bands has been linked to different brain functions. Generally, slow frequency bands are related to the coordination and integration of different areas in long time windows, while the highest frequency bands are associated with faster, more local information processing. Of the named frequency bands, those that take relevance in this thesis are theta and beta. Thus, they are briefly explained here.

Theta . Theta activity has been observed primarily in the hippocampus. It has been related to exploratory movements and spatial navigation task [29]. Furthermore, it has been proposed that theta rhythm is involved in sensorimotor integration, providing continually updated feedback to the motor system regarding their performance relative to changing environmental (sensory) conditions [30].

Theta oscillations are also often linked to high-level cognition like memory, emotional regulation and working memory.

Beta . Beta band is commonly related to sensorimotor behaviour. It is known that beta band power decreases during the preparation and execution of voluntary 
movement, bursting after the finishing of the movement [31]. It has been found that this decrease in beta activity does not occur only during the execution of movements, but also if such movements are only imagined, not being accompanied by any muscular activity. This fact is exploited by the BCI (Brain-Computer Interface) systems for the control of robotic arms from the neuronal signal $[32,33]$.

\section{Electrophysiology}

The complex system that the brain supposes has been studied throughout our history under different approaches. Currently, methods have used that try to characterize brain activity through electrophysiology techniques. Electrophysiology is concerned with the study of electrical activity in the human body [34], in our case, within the brain. There are different electrophysiology techniques, these differ in terms of: 1)The signal recorded, which could be electric potentials or magnetic fields and 2)The recording site. The measures can be invasive (the electrodes are positioned on the brain surface, or even inserted into deep brain structures, as in the studies of the third block of this thesis) or non-invasive (the electrodes are outside the brain, in direct contact or not with the head). The main neuroelectrophysiology techniques include:

Electroencephalografy (EEG) . EEG measures the electrical activity of the cerebral cortex by means of a series of electrodes (range from 19 to 256) which are placed over the scalp. To reduce skin-electrode impedance, the application of conductive gel is necessary. The main advantage of this technique is that it is inexpensive. One of the main applications of EEG is in the diagnosis and typification of the different types of epilepsy, some types of dementia, and the determination of brain death.

Electrocorticography (ECoG) . ECoG is based on similar principles than EEG, but in this case, the signal is obtained using a mesh of electrodes placed over the cerebral cortex, after performing a craniotomy. This gives a better spatial resolution than EEG. ECoG is currently considered as the standard system for defining epileptogenic zones in clinical practice.

Magnetoencephalography (MEG) . MEG is one of the most modern neuroimaging techniques. It captures the magnetic field generated by the electrical activity of neurons. MEG is an effective technique for studying cortical activity, being able to collect the neural activity directly with a very high temporal resolution and a good spatial resolution.

Intracraneal Electrodes . By using intracranial electrodes, it is possible to register local field potentials (LFP), which measures the aggregate presynaptic and postsynaptic activity of a population of neurons, as explained below. LFP provides one of the best sources of information with regard to brain activity since they enable 
us to observe the original signal generated within deep structures without the need to apply a mathematical algorithm of source reconstruction.

Electromiography (EMG) . Although EMG is not a neuroelectrophysiology technique, it is directly related. It is also one of the electrophysiology techniques used in this thesis, which is why we include it in this list. EMG registers, by means of a needle electrode, the electrical activity of a muscle. It is used to evaluate the health of muscles and the neurons that control them (motor neurons). Motor neurons transmit electrical signals that cause muscles to contract. This technique is usually used to reveal possible nerve dysfunctions, muscle dysfunctions or problems in the transmission of nerve signals to muscles.

Medical imaging techniques . Electrophysiological methods measure neural activity. But there are a number of techniques widely used in neuroscience studies to measure neural function, such as PET, fMRI or fNIRS. Positron emission tomography, also called PET imaging, is a type of nuclear medicine imaging, which generates 3D images of metabolic activity of the brain. Functional MRI (fMRI) allows the active brain regions to be shown in images. The most common fMRI technique is BOLD-contrast imaging, which reflects local levels of oxygen in the blood at each point in the brain (increased oxygen levels in an area are associated with increased neuronal activity in that area due to the hemodynamic response generated by neurovascular coupling). Functional near-infrared spectroscopy (fNIRS) using low levels of non-ionizing light to measures hemodynamic response through optical sensors placed on the surface of the scalp.

\subsection{Intracraneal Recordings}

T f we need to monitor the activity of a small population of neurons, extracellular $\perp$ physiology is currently the best technique. Using electrodes, we can measure the activity of few cells (Spiking Activity - SA) or instead sense the activity of a larger group of cells (Local Field Potentials - LFP) [35]. In the closed-loop deep brain stimulation works presented in this thesis (chapters [5-8]) we have worked with LFP registers.

LFP is a massed neuronal signal. The scientific community has reached a consensus to use the term local field potential for signals recorded by small intracranial electrodes [36], as opposed to the signal collected by external electrodes such as EEG or MEG. LFP represents information of integrative excitatory and inhibitory synaptic processes at the level of neural population activity. The electrodes sense and average the field potentials that are in their neighbourhood [37]. Thus the positioning of electrodes has to be very accurate in order to prevent a particular cell dominating the electrophysiological signal. Note that LFP is a signal composed of the activity of a population of cells, which range in number from a few hundred to thousands. An example of a LFP recording is shown in 4.1 
An interesting point about LFPs is that they represent a more stable signal in time than other neuronal signals such as multiunit spiking activity. This makes it a good signal for clinical applications based on BCI [38]. However, LFP is more difficult to interpret that spikes, since multiple processes may be contributing to the signal. It is important to note that LFPs not only reflect the temporal pattern of spike activity of the neurons near the electrodes but also the incoming currents.

In the case of this thesis, the electrodes are positioned on the STN. So we have the signal not only from the STN, but also from the contribution of the basal ganglia circuit. It is possible that this makes the signal richer in information, endowing it with characteristics that help in the distinction of tremor states.

\subsection{Magnetoencephalography}

$M$ agnetoencephalography (MEG) is a completely non-invasive technique that Measures the magnetic fields derived from currents generated in the brain as a result of brain activity. It presents several advantages comparing with other techniques. Comparing it with electroencephalography (EEG) [39]: 1) The magnetic field is less affected by tissues like the skull and scalp 2) MEG does not need a reference electrode, which is beneficial for functional connectivity (FC) studies and 3) MEG recording is made up of about 300 channels, a number far greater than that found in EEG, thus increasing the spatial resolution. If we compare MEG with electrocorticography (ECoG), we find that both techniques have similar values of temporal and spectral resolution; however, ECoG is a much more invasive technique. Finally, MEG provides us with a sub-second temporal resolution, which is the main limitation of functional magnetic resonance imaging (fMRI). All these reasons make MEG one of the most appropriate techniques for studying the human brain. Because of it, it is one of the techniques chosen together with LFPs in this thesis (in the study presented in chapter 3). Given the high level of complexity of this technique, its principles are introduced in this section.

\section{MEG Hardware}

The magnetic field generated by brain activity, which we want to measure, is very low intensity. They are in the order of 50-500 fT (10-15 Tesla), around 100 million times weaker than the magnetic field of the earth. The environmental magnetic fields generated by e.g. computers, cables or cars are far more intense than the neuromagnetic fields. Therefore, the MEG system is placed in a shielded magnetic room, as shown in figure 2.3. The walls of the room are made of aluminium and mu-metal, a nickel alloy. In this way the walls have high magnetic permeability (much more than the interior of the room), acting as a conductor of the external magnetic fields and preventing them from entering the room.

To capture the tiny neuromagnetic fields, MEG system uses a set of superconducting quantum interference devices (SQUIDs), designed to measure extremely small magnetic fields. These devices turn magnetic flux, collected by sensors into volt- 
a)

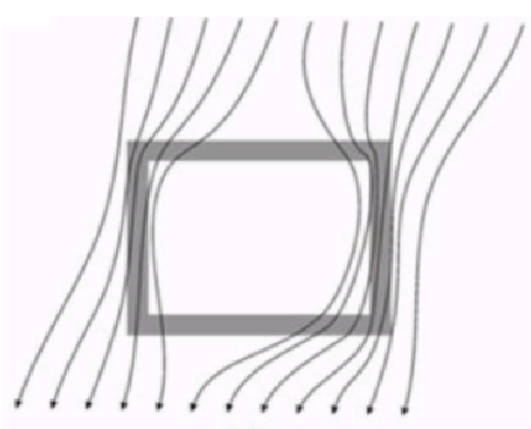

b)

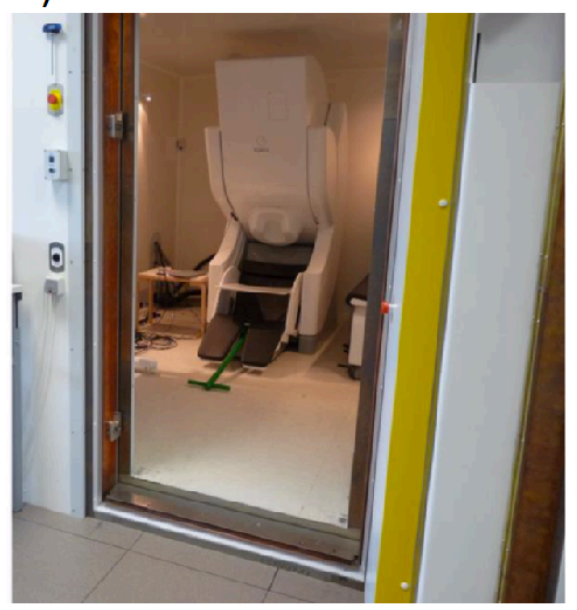

Figure 2.3: a)The aluminium and mu-metal layers of the shielded room create a path for magnetic field lines around the enclosure. b)Magnetic shielded room of Elekta MEG system at the Center for Biomedical Technology (Technical University of Madrid).

age. The MEG systems contain around 300 SQUIDs which are connected to sensor coils in an assembly that follows the curvature of the head, as depicted in figure 2.4. There are two types of sensors that pick up the magnetic field: magnetometers and gradiometers. Gradiometers are composed of two coils placed, in the case of MEG, in parallel (there are gradiometers with coils placed in series). These planar gradiometers calculate the partial derivative of the magnetic field passing through the sensor perpendicular to the measured magnetic field. These sensors mainly measure sources that are placed underneath them, losing sensitivity to other sources, which is an advantage over volume conduction when studying the signal in the sensor space. For their part, magnetometers consist of one coil which senses the magnetic fields perpendicular to the coil's surface. Unlike gradiometers, they are capable of recording the magnetic field from both surface and more in-depth sources. Both types of sensors must be maintained at $-269^{\circ} \mathrm{C}$, needing a cooling system that works with liquid helium.

\section{MEG Preprocessing}

The magnetically shielded room is an essential element in the mitigation of external magnetic fields. However, it is not a sufficient condition, since more artefacts contaminate the recordings. They can be physiological or non-physiological in origin. For the MEG analysis to be valid, the signal must be clean, i.e. without any artefact. To this end, several preprocessing procedures have to be applied to correct or eliminate the contaminated segments. This section introduces the preprocessing pipeline. 


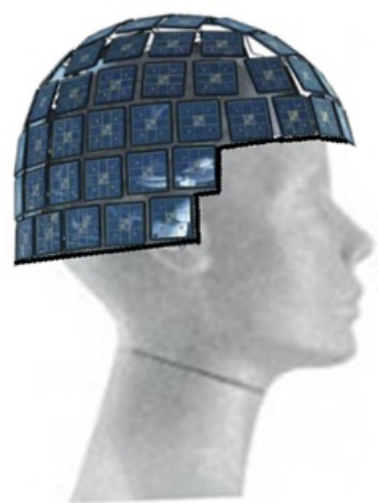

Figure 2.4: MEG Helmet; composed of SQUIDs, connected to sensor coils following the curvature of the head.

tSSS

Temporal Signal-Space Separation (tSSS) [40] is used to filter magnetic fields coming from outside the head. It is the temporal extension, i.e. adding the time dimension, of the SSS procedure and is implemented by Elekta Neuromag MEG systems, within MaxFilter software.

SSS is based on the principle that the generated magnetic fields inside the head surface present a different spatial distribution than those who come from outside. In accordance with Gauss theorem, a signal generated on a closed surface can be modelled using a series of tangential currents on the surface of this closed surface. Applying this theorem, two concentric spheres can be defined. One sphere models the signal that comes from brain physiological signals and is defined inside the MEG sensors array. While the second sphere models the sources of noise and it is defined outside the sensor, as depicted in figure 2.5. The components originating in the sphere inside the head are projected into the sensor space. Thus, correlations are sought between internal and external components. Since the two signals should not be related, components with a high degree of correlation are discarded.

\section{Artefacts Removal}

Among the most important physiological artefacts, we find eye artefacts, muscle movements or tensions, and even the heartbeat itself. We consider these signals to be artefacts, as they are not the signal of interest in the study, but which nevertheless interfere in our MEG recording. Spatial filters like tSSS cannot be employed to remove these artefacts, being necessary to apply other methods.

- Muscular Artefacts. Are due to muscle contraction. They occur at high frequencies, in the beta and gamma bands. They are usually due to jaw movements or muscle tension in the neck and shoulders. Therefore, these artefacts are usually located in frontal and temporal sensors.

- Ocular Artefacts. Two types of ocular artefacts can be found, eye movement 


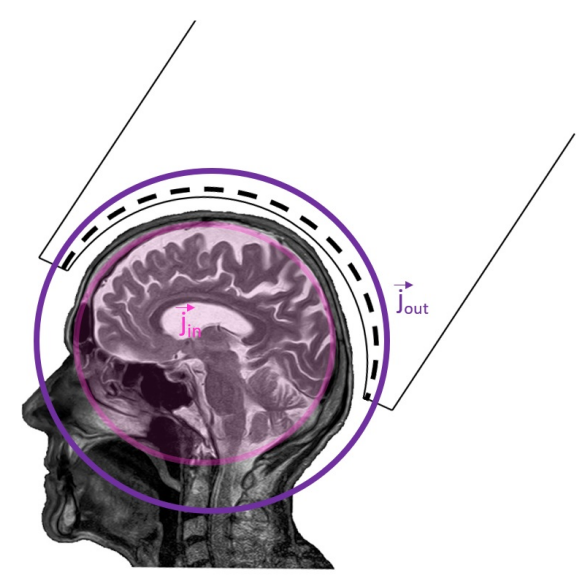

Figure 2.5: Model for SSS

and blinks. Both are related to eye muscle movement. When the eye moves, it generates a variable magnetic field, detected in the frontal sensors. Eye blinks produce a current that can be easily identified by its peak shape at frequencies around $2-3 \mathrm{~Hz}$, thus severely contaminating the delta and theta bands.

- SQUIDS Jumps. These artefacts are due to SQUIDS electronics. They produce a sudden change in the amplitude of the signal, taking on a very narrow square wave appearance (hence the name jump).

In order to remove them, automatic tools are used to detect the segments of the recording that contain some of these artefacts. In this thesis the function ft-artifactzvalue, belonging to Fieldtrip toolbox has been used for this purpose [41]. Given the high number of false positives returned by the automatic tool, a visual inspection of the detected artefacts is necessary. During this visual inspection, the real contaminated data segments are identified, avoiding false positives. These segments are discarded since it is not possible separating them from the neurological signal of interest.

MEG recordings are usually complemented with auxiliary physiological channels, like electrocardiogram (ECG) or electrooculogram (EOG). In these cases, the signal from these channels can be used to eliminate artefacts by means of Independent Component Analysis (ICA). ICA is a computational method that separates a multivariate signal into additive subcomponents. Some of these components will contain noise sources. Taking the cardiac component as an example, comparing the components decomposed by ICA, with the ECG channel, it will be possible to identify the component(s) that most represent the cardiac signal. These components will be removed from the signal. In the same way, this procedure can also be used to eliminate eye blinks, in case of an external EOG channel is available. 


\section{Source Reconstruction}

Once the signal is clean of noise and artefacts, it can be analysed in the sensor or the sources space. Analysing the data in the sensor space means that the features will be extracted from the signal acquired in each of the MEG sensors (magnetometers and/or gradiometers). Although it is a valid procedure, it is sensitive to volume conduction, due to the fact that each sensor registers activity from different sources. Hence, some spatial resolution is lost in the analysis.

A more precise approximation is the analysis in the source space. In the past years, better source localisation mathematical algorithms have been proposed. These methods are robust to noise, informed by anatomy (using the magnetic resonance imaging (MRI) of the subject) and use realistic volume conduction physics. The objective is to obtain the time-series related to the activity in each of the defined neuronal sources. This approach partly solves the problem of volume conduction, although it is not exempt from source leakage. Source leakage implies that at the source level, due to the spatial resolution of the data, neighbouring sources may share some activity [42].

In order to acquire the signal in sources, the forward (or direct) and inverse (or indirect) problem must be solved:

Forward Problem . Before reconstructing the time series in sources, it is necessary to solve the forward problem: given a certain current located in the brain, we need to find which magnetic field is registered by the sensors. For this, it is necessary to calculate how the magnetic fields propagate through the different surfaces within the head. To this end, a conduction model is constructed, which consists of different layers of homogeneous conductivity. On this model, the Maxwell equations are applied, as explained in this section [43].

Maxwell equations are a set of equations that fully describe electromagnetic phenomena. Assuming a linear, homogeneous, isotropic and non-dispersive propagation medium, a relationship can be found between the electrical intensity and magnetic induction vectors through two parameters known as electrical conductivity $\sigma(\vec{r})$ and magnetic permeability $\mu(\vec{r})$. Applying the Biot-Savat law, the magnetic field $\vec{B}$ at the spatial point $\vec{r}$ is determined by:

$$
\vec{B}(\vec{r})=\frac{\mu_{o}}{4 \pi} \int_{V} \vec{j}(\vec{r}) \times \frac{\left(\vec{r}-\vec{r}^{\prime}\right)\left\|\vec{r}-\vec{r}^{\prime}\right\|^{3}}{d} \vec{r}
$$

where $\vec{r}$ is a point in the space $V$, which encloses the currents $\vec{j} . \vec{j}$ represents both intracellular $\vec{j}_{p}(\vec{r})$ and extracellular $\vec{j}_{v}(\vec{r})$ currents. We are interested only in $\vec{j}_{p}(\vec{r})$, which represents the neuronal activity. Forward modelling consist in calculate $\vec{B}(\vec{r})$ knowing $\vec{j}_{p}(\vec{r})$. 
To this end, the Leadfield matrix $\vec{L}$ is calculated, which consists of a filter or mask that relates $\vec{j}_{p}(\vec{r})$ with the activity registered in the MEG sensors, following equation:

$$
m_{k}=m\left(\vec{r}_{k}\right)=\int_{v} \vec{L}\left(\vec{r}_{k}, \vec{r}^{\prime}, \sigma\left(\vec{r}^{\prime}\right)\right) \cdot \vec{j}_{p}\left(\vec{r}^{\prime}\right) d^{3} \vec{r}
$$

In this thesis, we have employed the Boundary Element Method (BEM) in the solving of forward modelling. We have defined three isotropic and homogeneous conductivity layers: the brain, skull and scalp, by using the T1-weighted MRI of each subject. This choice is based on the fact that the 3-shell BEM method has been shown to estimate leadfield matrix realistically.

Inverse Problem . In reverse, the inverse problem consists of calculating the brain currents that originated the magnetic field measured on the surface (by the sensors). It does not exist a unique solution to this, since several distributions of cerebral currents, can result in the activity measured in sensors. It is therefore said that the inverse problem is ill-posed. We, therefore, want to find a solution to the inverse problem, even if it is not the only possible one. For this, it is necessary to assume a priori assumptions that limit the number of solutions to only one. These assumptions will depend on the mathematical model chosen in solving the inverse problem. In this thesis we have worked with Beamforming method.

Beamforming methods were originally developed for its use in sensor arrays for directional signal transmission or reception. Beamformers are adaptative spatial filters which estimate the activity of the dipoles so that the activity of each one is independent of the rest. More specifically, the activity in a dipole is calculated as a linear combination of the signal recorded in the sensors, determined by the filter value $w$ (where there is a value $w_{i}$, for each sensor $k_{i}$ ) [43]:

$$
j_{i}=\sum_{k=1}^{N_{\text {sensors }}} w_{k} \cdot m_{k}
$$

The perfect beamforming filter is the one that reconstructs in a certain point $\vec{r}_{i}$ the signal $j_{i}$ with gain 1 , and 0 in the rest of points.

\subsection{Analysis Methods}

Throughout this thesis, many methods of analysis have been studied, implemented and applied to the DBS study. These methods are introduced in this section, but the parameters used and the detailed description of how they have been used will be detailed in the different chapters (corresponding to the different published papers) of the thesis.

\subsubsection{Spectral Analysis}

A fundamental analysis that can be done on the neuronal signal is spectrum analysis. The underlying idea is that a complex signal is a composition of sinuous waves 
at different fundamental frequencies. Fourier Transform (FT) is the classical way to decompose a signal into the frequencies it is composed. It is computed convolving the signal with the sine waves. The number of sine waves and their frequencies will be determined by the number of data points in the signal. The original definition of FT assumes that the signal is stationary; that is, its mean, variance and frequency do not evolve over time. This is not the case in the neural signal, which results in a blurring of the frequencies present in the signal. This effect is called spectral leakage, and it is one of the main reasons to employ temporally localised frequency decomposition methods, such as short-time Fourier transform (STFT) or Wavelet analysis. In work presented in the chapter 4 we have been used the Wavelet transform, so it is presented here.

The Continuous Wavelet Transform (CWT) is defined as:

$$
X_{f}(a, b)=\frac{1}{\sqrt{a}} \int_{-\infty}^{+\infty} f(t) \cdot \psi^{*}\left(\frac{t-b}{a}\right) d t
$$

The results of the CWT are many wavelet coefficients, which are function of scale (a) and position $(b)$, where $\{a, b\} \in \mathbb{R}$. The scale can be viewed as a compression factor, and it is linked to the frequency. Low scale $a$ values (compressed Wavelet) correspond to high frequency, and high scale $a$ values (stretched Wavelet) are equivalent to low frequency. The position factor represents the delay of the signal. $\psi$ is the basic wavelet function, the so-called mother wavelet (e.g., daubechies, biorthogonal, symlets, etc.), and the asterisk * represents the operation of the complex conjugate. The wavelet transform decompresses the signal into different scales with different levels of resolution by stretching (or compressing) the mother signal.

The calculation of wavelet coefficients at any value of scale $(a)$ and position $(b)$ is often redundant and requires a high amount of work. The analysis can be done more efficiently if scales and positions are a power of two (dyadic scales and positions), which is called the Discrete Wavelet Transform (DWT). In CWT, if $a=2^{m}$ and $b=n \cdot 2^{m}$ and $\{m, n\} \in \mathbb{Z}$ we obtain the following equation:

$$
\begin{aligned}
X_{m, n} & =\int_{-\infty}^{+\infty} f(t)\left[2^{-m / 2} \cdot \psi\left(2^{-m} \cdot(t-n)\right)\right] d t= \\
& =\int_{-\infty}^{+\infty} f(t) \cdot \psi_{m, n}(t) d t
\end{aligned}
$$

Wavelets can be calculated by iteration of filters with rescaling. Two sets of coefficients are generated at each stage: approximations coefficients $Y_{j, k}$ and detailed coefficients $X_{j, k}$. In detail, these vectors are obtained by convolving $f(t)$ with a low-pass filter $h_{0}$ and a high pass filter $h_{1}$, followed by dyadic decimation - only half the number of samples represents the signal. 


\subsubsection{Synchronization Analysis}

In the XIX century, neuroscience postulated a model of the brain based on segregation. Under this paradigm, different physical areas of the brain specialise in different functions. An example is Broca's area, which is a section of the human brain located in the third frontal circumvolution of the left hemisphere. The French doctor Paul Pierre Broca described it in 1864, after observing several post mortem aphasic patients who presented severe damage in that region. Analogously, cognitive or sensory impairs produced by damage in different areas were studied, thus deducing the function of that area.

Segregation model is being completed by modern neuroscience with the brain integration model. Under this paradigm, the areas are not isolated but are interconnected and relate to each other to carry out different brain processes, through its synchronisation. Nowadays we know that the brain needs the synchronisation of different areas for its correct operation.

The analysis of synchronised activity is a relatively novel approach to employ when observing the functioning of the brain. Methods of brain connectivity can be classified into three categories: Anatomical, Functional (FC) and Effective (EC) connectivity. Anatomical connectivity methods study the physical connections within the architecture of the brain, i.e. the forest of tracts that physically link brain areas. FC methods quantify the statistical dependence between temporal series generated by different neuronal units (from single neurons to larger neuronal areas). FC are symmetrical methods, so they do not give information on directionality. i.e. no causality can be derived from the analysis. On his side, EC methods rely on the assumption that, when studying brain connectivity, true interactions between two neural sources appear with a certain time delay, the time in which the information is travelling [44]. Thus, these methods can detect this directionality, providing causal information.

It is important to note that the existence of FC or EC between areas does not imply their physical connection, only supposes a relationship between the signals they generate. In this way, by applying these methods, it is possible to study phenomena in which different areas of the brain are implicated, not being physically connected. This involves possibly more complex and unknown phenomena.

\section{Hilbert Transform}

Before describing the different connectivity methods, it is necessary to introduce the Hilbert transform. As introduced above, an oscillatory neural signal can be divided into frequency bands. From the resultant narrow-band signal $x(t)$, we can extract the phase $\varphi_{x}(t)$ and the amplitude $A_{x}(t)$. The phase indicates the instantaneous situation within the cycle, of a magnitude that varies cyclically. The amplitude, also called envelope, is the magnitude of power that the wave has at every instant. The amplitude of the signal varies less than its phase in time. It is, therefore, easier to find amplitude coupling between two signals, without the need for them to be 
coupled in phase.

For phase and amplitude calculation, the analytical signal of the Hilbert transform is usually used since it is computational and mathematically simpler than other methods such as Wavelet transform. Note here that Hilbert should only be employed in narrow-band signals (while wavelet can be applied in broadband signals). The analytical signal is computed as:

$$
s(t)=x(t)+i H_{x}(t)=A_{x} e^{i \varphi_{x}(t)}
$$

where $x$ is the original signal, $i$ is the imaginary unit, $H_{x}(t)$ is its Hilbert transform, which is defined as:

$$
H_{x}(t)=\frac{1}{\pi} p \cdot v \cdot \int_{-\infty}^{\infty} \frac{x(t)}{t-\tau} d \tau
$$

where p.v is the Cauchy principal value.

There are several mathematical methods to estimate connectivity, which can be grouped into different families. In this thesis, I have used two classical linear methods (Coherence and Amplitude Envelope Correlation), two information-theory based methods (Mutual Information and Phase Transfer Entropy), a phase synchronisation method (Phase-Locking Value) and a high-order spectra method (Bicoherence). All are FC measures, except Phase Transfer Entropy, which belongs to EC methods. The mathematical description of all of them is presented below.

\section{Classical Methods}

The family of classical methods encompasses the approaches that have traditionally been applied in the neuroscience literature, such as Coherence, Pearson's correlation coefficient and cross-correlation. In this thesis, I have made use of coherence and Amplitude Envelope Correlation. Although the latter is not a classical method, I have included it within this family as it is based directly on the correlation method.

Coherence . The coherence function estimates the linear correlation between two signals $x$ and $y$ as a function of the frequency. It is defined as the crossspectrum $C_{x y}(f)$ normalised by the product of the individual power spectral densities (auto spectrum) of each signal, $C_{x x}(f)$ and $C_{y y}(f)$ [45]:

$$
\Gamma_{x y}(f)=\frac{C_{x y}(f)}{\sqrt{C_{x x}(f) C_{y y}(f)}} ; 0 \leqslant\left|\Gamma_{x y}(f)\right| \leqslant 1
$$

I used Welch's averaged, modified periodogram method to estimate the auto and cross spectra since we are dealing with a finite amount of data. 
Amplitude Envelope Correlation . Amplitude envelope correlation (AEC) is an index developed to detect signal-coupling without phase coherence. It can detect synchronisation in a less precise coupling of signals than coherence since the envelope of band-limited signals does not change as rapidly as the signals themselves [46]. This type of measures (envelope correlations) are interesting because they are related to fMRI BOLD (Blood-oxygen-level-dependent) signal amplitudes [47].

Given two signals $x$ and $y, \mathrm{AEC}$ is the mean correlation of their envelopes. We calculate AEC as:

$$
A E C=\operatorname{corr}\left(\left|x_{h}\right|,\left|y_{h}\right|\right)
$$

where $x_{h}$ and $y_{h}$ are the Hilbert analytical signals of $x$ and $y$ respectively.

\section{PS Methods}

Phase synchronization indexes (PS) are used to investigate if the phases of two oscillators are coupled, even if their amplitudes may not be [48]. If two systems present PS, it means that the difference of their phase over time remains bounded:

$$
\phi(t)=m \phi_{x}(t)-n \phi_{y}(t)=c t e
$$

, where $m$ and $n$ are integer values which define the different types of coupling. Normally $m=n=1$ is used, in this case, the phase difference between the signals is fixed.

Physiological recordings contain some noise, holding random phase slips of $2 \pi$. So in practice, we work with the relative phase difference wrapped to the interval $[0,2 \pi)$, known as cyclic relative phase:

$$
\Delta \phi_{\text {rel }}(t)=\Delta \phi(t) \bmod 2 \pi
$$

Phase-Locking Value . The preferred index to observe this phenomenon in neuroscience is arguably the Phase-Locking Value (PLV), which measures the relative phase difference between $x$ and $y$ and estimates how it is distributed over the unit circle [49]. It is defined as:

$$
P L V_{x, y}=T^{-1}\left|\sum_{T} e^{i\left[\phi_{x}(t)-\phi_{y}(t)\right]}\right| ; 0 \leqslant P L V_{x, y} \leqslant 1
$$

where $\phi_{x}$ and $\phi_{y}$ are the phases of $x$ and $y$, extracted from the Hilbert analytical signal, respectively, wrapped to the interval $[0,2 \pi)$. Thus, we work with the cyclic relative phase, avoiding the phase slips of $2 \pi$ that may be present in the signal. We get a value of one when the phase difference across windows is the same, and a value of zero when it varies randomly.

\section{Information Theory Based}

Mutual Information . Mutual Information (MI) is one of the most used indexes of interdependence based on information theory. MI draws from Shannon's concept of entropy [50], which can be regarded as the amount of information a variable 
holds. Therefore, it is also a measure of its uncertainty.

Given a random signal $x$, with a probability distribution $p(x)=P\{X=x\}, x \in X$, its entropy is defined as:

$$
H(X)=-\sum_{x \in X} p(x) \log p(x)
$$

Joint Entropy is the extension of the concept of entropy to two variables. The joint entropy for a couple of discrete random $X$ and $Y$ signals is:

$$
H(X, Y)=-\sum_{x \in X}-\sum_{y \in Y} p(x, y) \log p(x, y)
$$

The joint entropy will always be less than or equal to the sum of the individual entropies: $H(X, Y) \leq H(X)+H(Y)$; equality being met only if they are independent variables.

Henceforth, for a pair of random variables, we can estimate the amount of information they share, which is the concept of MI:

$$
M I=\sum_{x \in X} \sum_{y \in Y} p(x, y) \log \frac{p(x, y)}{p(x) p(y)}
$$

where $p(x, y)$ is the joint probability. If $M I=0$, then the variables are independent. Otherwise (i.e., if $M I>0$ ), there exists some degree of interdependence between both signals [45].

Phase Transfer Entropy . Phase Transfer Entropy (PhTE) quantifies the directed interaction between two time series by applying the concept of transfer entropy to their phases [51]. It has the advantage, when compared with other directed methods such as Granger or Dynamic Causal Modelling, to be model-free (not making assumptions about the system) and works with only one parameter, thereby reducing the possibility of erroneous results due to the election of parameters. PhTE is defined as:

$\operatorname{PhTE} E_{x \rightarrow y}=H\left(\phi_{y}(t), \phi_{y}\left(t^{\prime}\right)\right)+H\left(\phi_{y}\left(t^{\prime}\right), \phi_{y}\left(t^{\prime}\right)\right)-H\left(\phi_{y}\left(t^{\prime}\right)\right)-H\left(\phi_{y}(t), \theta_{y}\left(t^{\prime}\right), \phi_{x}\left(t^{\prime}\right)\right)$

where $\phi_{x}\left(t^{\prime}\right)$ and $\phi_{y}\left(t^{\prime}\right)$ are the past states at time point $t^{\prime}=t-\delta: \phi_{y}\left(t^{\prime}\right)=$ $\phi_{y}(t-\delta), \phi_{x}\left(t^{\prime}\right)=\phi_{x}(t-\delta)$, and $H(\bullet)$ is the entropy and the joint entropy as defined previously in equations 4 and 5 .

\section{High-Order Spectra Methods}

Bicoherence . Power spectrum analysis is usually performed via Fourier Transform (FT), of the second-order statistic of the signal. However, unfortunately, this 
measure loses information about the phase relationships between frequency components, a fact that has been linked with impaired functions in the brain [52].

The bispectrum is a two-dimensional version of the FT based on the third-order cumulant of the signal. It is defined as:

$$
B\left(f_{1}, f_{2}\right)=\sum_{m=-\infty}^{\infty} \sum_{n=-\infty}^{\infty} R(m, n) e^{-j 2 \pi f_{1} m} e^{-j 2 \pi f_{2} n}
$$

where $R(m, n)$ is the third order cumulant as a function of the lags $m$ and $n$, and $f_{1}$ and $f_{2}$ are the frequencies in study.

The bispectrum can be used to investigate if the signals at $f_{1}, f_{2}$ and $f_{1}+f_{2}$ are synchronised, which would mean that the oscillation at $f_{1}+f_{2}$ is due to the nonlinear relationship between both signals. Bicoherence $(\mathrm{BICOH})$ can be calculated as a normalised version of the bispectrum [53]:

$$
\operatorname{BICOH}\left(f_{1}, f_{2}\right)=\frac{B\left(f_{1}, f_{2}\right)}{\sqrt{P\left(f_{1}\right) P\left(f_{2}\right) P\left(f_{1}+f_{2}\right)}}
$$

where $P(f)$ is the power spectrum at frequency $f$. For incoherent signals this measure tends to zero.

\subsubsection{Non-linear Dynamical Analysis}

Nonlinear dynamical systems theory, also known as Chaos theory, is the branch of mathematics and physics that deals with complex systems and nonlinear dynamic systems susceptible to variations in the initial conditions. It can be used to study complex neural networks. I have worked within this paradigm in one of the published studies. Here is a brief introduction to the basic concepts, the application of which can be seen in more detail in chapter 7 .

A dynamical system is a system that evolves over time and that such evolution can be defined establish from the initial state only. Two concepts emerge from this definition, state and dynamics. The state is defined by the values taken by the variables at a particular moment. Thus, the state defines the system at a given time. We call state space, also known as phase state, to the set of all the possible states that the dynamic system can take. While the dynamics are the set of laws that describes how the system evolves from one state to another, which usually consists of a system of as many differential equations as variables the system has [54]. A dynamic system can be defined from different perspectives:

- Linear vs Non-linear. The system is linear if the equations that describe it are linear. It is nonlinear otherwise.

- Conservative vs dissipative. A dynamical system is conservative if the values taken by the system variables are maintained over time, or seen graphically 
if the volume of state space is preserved. Otherwise, it is dissipative, and therefore, the volume of the state space decreases with time.

- Deterministic vs Stochastic. The system is deterministic if the laws that describe it do not contain any external source of randomness, and stochastic if they do.

Biological neural networks seem to behave as nonlinear dissipative systems. It is an open question of whether they are deterministic or stochastic [54]. In chapter 7 , this aspect is deepened in the case of the subthalamic signal during the tremor.

Two other important concepts of dynamic systems are the trajectory and the attractor. The trajectory of the system is the succession of states through which the system passes, which defines its dynamics. The attractor is the set trajectories towards which a system tends to evolve, given a wide variety of initial conditions in the system. We can find different types of attractors depending on the dynamic system that generates it. The attractor of a linear dissipative deterministic system will be just a point, in which the system will be in equilibrium (figure 2.6 a). This is the simplest type of attractor, but the nonlinear dissipative deterministic system can present more complex forms: (i) If the system exhibits a periodical behaviour, the attractor will be a closed-loop in the phase state (figure $2.6 \mathrm{~b}$ ). (ii) The attractor will be shaped like a torus (figure $2.6 \mathrm{c}$ ) in systems with quasi-periodic dynamics, in which the periodic behaviour is defined as recurring at regular intervals. (iii) Chaotic or strange attractor (figure $2.6 \mathrm{~d}$ ) corresponds to a system which exhibits deterministic chaos. Such a system, although deterministic, is susceptible to initial conditions. For this reason, if there is a small variation in the initial conditions, the trajectories will tend to diverge. However, given that the system is bounded at some point begins to converge. This process is responsible for the generation of attractors with more complex shapes than the previous ones.

The state-space trajectory of a dynamical system can be reconstructed from a scalar time series, by taking $m$ time-lagged observations of it. This is the well-known Taken's embedding theorem; it will be explained more in detail in Chapter 7 where it is applied to our problem.

Characterising state space properties is equivalent to characterising the dynamics of the system. There are several ways to do this analysis. One of the first methods was the Lyapunov exponents that characterise the complexity of the attractor indicating the exponential convergence or divergence of nearby trajectories of the attractor. Another classical technique is to analyse the recurrence plot (RP) of the system. RP is used to visualise the recurrences of trajectories in the phase space of a dynamical system by looking at the patterns presents in the plot. However, observing patterns by eye can sometimes be complicated. Therefore, there are a set of mathematical measures which can help in the classification of the patterns presents in the RP called recurrence quantification analysis (RQA), such as recurrence rate, per cent determinism, entropy, average and maximum diagonal length, 


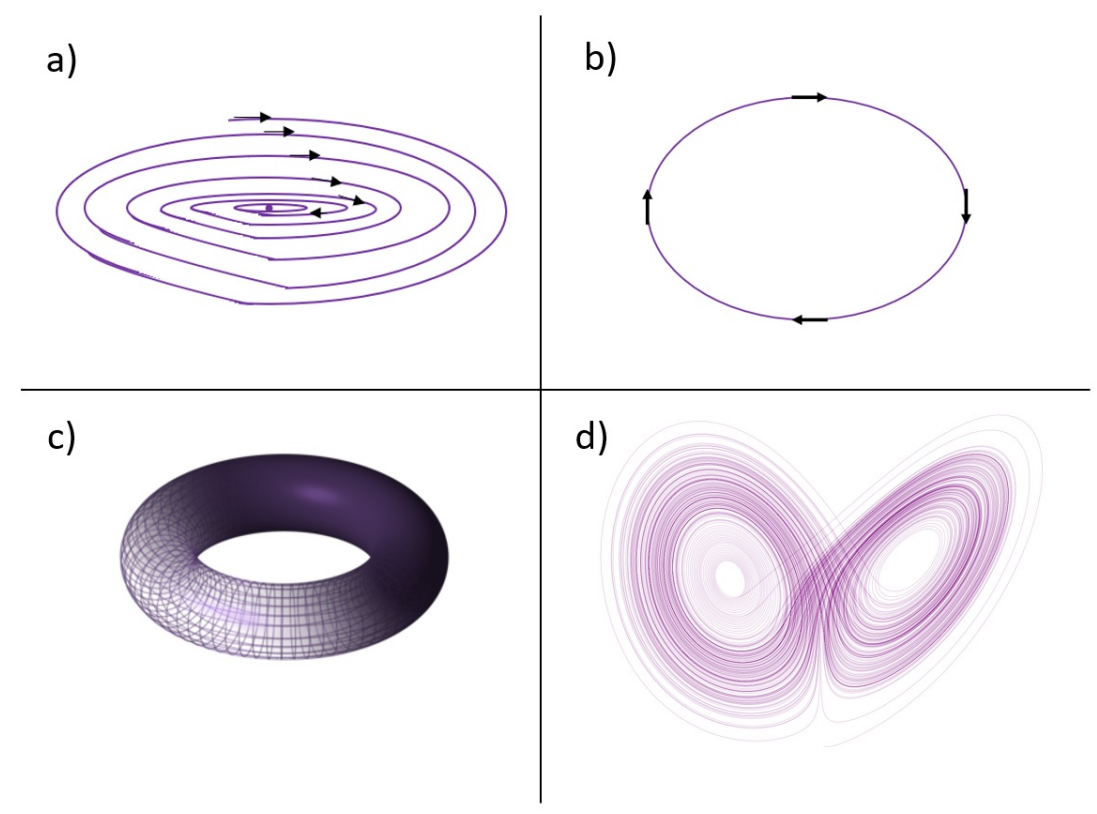

Figure 2.6: Attractor of a: a)linear dissipative deterministic system. b)system with a periodical behaviour. c)system with a quasi-periodic dynamics. d)system with deterministic chaos.

ratio and laminarity. See [55] for more information.

The transformation of time series into complex networks for nonlinear time series analysis has recently gained relevance. The objective is to quantify the topology of the network using mathematical measures from graph theory. These methods include proximity networks, visibility graphs and transition networks. Proximity networks are calculated from the mutual closeness between different state vectors in the state space. In this thesis, we have worked with $\epsilon$-recurrence networks (RNs), a type of proximity networks, which are based on the recurrences in state space. They are obtained by reinterpreting the recurrence matrix as the adjacency matrix of a complex network. Mathematical details on the calculation of the $\epsilon-\mathrm{RN}$, as well as network analysis measures used in the non-dynamic analysis of the neuronal signal can be seen in chapter 7 .

\subsection{Statistical Methods}

nce the relevant features of the signal have been extracted, mathematical tools are needed to infer knowledge from them. To this end, several methods have been used in this thesis. While they are all statistical methods at a low level, we can classify them at a high level into statistical and machine-learning methods.

In this thesis, four systems for demand-driven stimulation have been proposed. 
The objective is to achieve a system that achieves $100 \%$ accuracy in the detection of tremor through the subject's subthalamic signal. This requirement is fulfilled in the last study carried out, corresponding to chapter 8 of the thesis. In this search for the perfect system, a wide variety of methods from the machine learning (ML) branch have been employed, which include artificial neural networks, clustering techniques, fuzzy-logic and data stream mining. Therefore, a brief introduction to $M L$ is included in the section 2.4.1.

For its part, in the MEG study, described in chapter 3, use is made of a statistical test called Cluster-based permutation test (CBPT). This method is explained in section 2.4.2.

\subsubsection{Machine Learning}

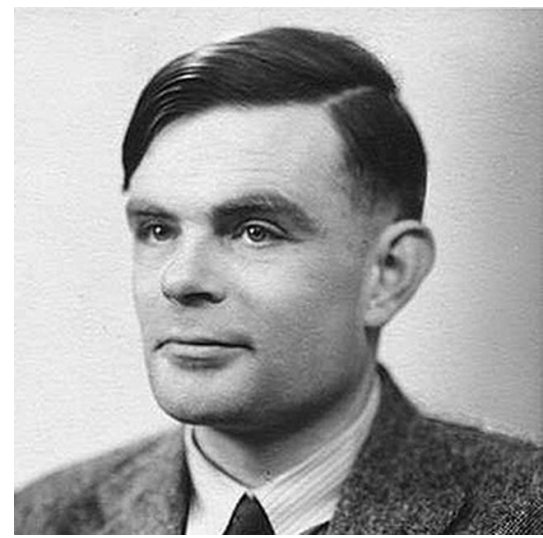

Artificial Intelligence (AI) is "the science of making machines do things that would require intelligence if done by a human", according to Marvin Minsky. However, long before this well-known definition, Alan Turing considered the father of computer science (CS) and artificial intelligence, proposed in 1950 the "Imitation game", today known as the Turing test. The objective of Turing was to determine "Can a machine think?", or even, "Is a machine intelligent?" [56]. The original test consisted of chatting (through typing on a keyboard) with a computer for five minutes. If after this time the interlocutor cannot discern whether or not he has been talking to a human, then it can be said that the machine has the same intelligence that a person could have.

Since then, the field of artificial intelligence has developed a lot, and now it is an entire branch of Computer Science that encompasses a variety of sub-fields such as Planning, Computer vision, Natural language processing (NLP) or Robotics among others. However, the branch of AI that has gained the most attention in the last years is Machine Learning (ML). The reasons are the growing demand for data analytics by both science and business, given the increase in the volume of data available today and what appears to be the end of the last "AI winter" with the development of new methods and a growing interest in this field by large computer science companies.

Analysing massive data (big data analytics), often in real-time, requires 1)An infrastructure of distributed systems which include storage, ingestion and extraction tools (like for example Apache Hadoop or Apache Spark) to store and share infor- 
mation efficiently, and 2)A set of mathematical methods that learn from such data, already available in data lakes. Meeting the first requirement is the goal of Big Data, a branch of CS (within the field of Systems Architecture) that takes care of highperformance computing. While the second requirement is the goal of Data Science, a branch of CS (within the field of $\mathrm{AI}$ ), which include data cleansing, preparation and analysis (using ML). Each of these two stages into which we can divide the data analysis process has its own environments and programming languages. In the development of this thesis, we have not been fortunate enough to have a big data infrastructure, so we have worked "only" in the second part of the process, data science.

ML develops algorithms capable of learning, that is, searching for knowledge in datasets that can contain hundreds of variables. To delve into the description of the different ML methods and its applications would constitute a thesis in itself. The mere objective here is to provide a quick overview of $M L$, given that it is present in all the published papers of this thesis. The details of the used methods are described in the corresponding chapters.

According to the classical classification, ML methods can be grouped in:

- Supervised learning: Supervised learning models are trained with a set of labelled examples, i.e., the class of each of the instances that are part of the training set is known. The training consists of adjusting a series of weights of the algorithm so as to minimise the error produced between the output produced by the algorithm and the real class of the instance. Once trained, the system will be able to determine the type of a new instance, not previously seen by the system. The two main applications of these models are classification and regression, which are distinguished in the type of the output variable, which is categorical in the first case and numerical in the second. Some of the classical supervised algorithms are Decision trees, Artificial neural networks (ANNs) or Support vector machines (SVM), among others. Today, with the boom in the application of datamining due to the large amounts of data available, new methods such as deep learning networks and ensemble (sets of classifiers) methods have emerged.

In this thesis, we have work with multilayer perceptrons (MLP), and with fuzzy logic, which is mathematically described in chapters 5 and 6 respectively.

- Unsupervised learning: Unsupervised methods of analysis are applied in unlabelled datasets. The goal is to discover structures or patterns in the data in order to find some type of organisation that helps in the analysis. The two main applications of these models are clustering and dimensional reduction. There are families of clustering algorithms, the choice of the correct algorithm must be made depending on the number of variables, the number of classes or the type of result to be obtained. The most known and basic clustering algorithm is K-means; which can be seen in chapter 5. Additionally, we have work with a stream clustering algorithm, encompassed within the data 
stream mining paradigm (different as far as the treatment of the data to the data mining). Its description can be seen in chapter 8 .

- Reinforcement learning ( $R L): R L$ is a paradigm of seeking to determine what actions an agent should choose in a given environment in order to maximise some reward. In $\mathrm{ML}$, the environment is generally formulated as a Markov decision process. Thus, the model consists of a set of states, a set of actions, a set of transition rules between states and rules that determine the rewards. Thus, the algorithm works like an automaton that chooses the rules that are triggered at each moment so that the reward is optimised and the final objective is fulfilled, extending different optimisation algorithms for it. This paradigm is applied mainly in robotics and games, but also in deep learning, personalised recommendations, or any field in which optimisation is sought.

\subsubsection{Cluster-based permutation test}

MEG signal is sampled at multiple sensors/sources and time points. Thus the MEG data has a structure of various dimensions. In our case, we acquire a spatio-spatiotemporal data structure. In this way, connectivity values are estimated between sources per each time point. Since the records are acquired in a resting state, the temporal dimension does not provide information. Therefore the connectivity values are averaged over this dimension. However, we still have a spatio-spatio structure, denoted as sample henceforth.

Our MEG study aims to determine the effects that DBS has at the cortical level. We do not start from a priori knowledge about these effects, but we want to study all the differences observed in the whole brain in the conditions of DBS-ON and DBSOFF. This implies a high number of statistical comparisons, one per each sourcesource pair. In this scenario, it is not possible to control the Family-wise error rate (FWER). This is the well-known multiple comparison problem (MCP). CBPT is a nonparametric method that controls the FWER at critical alpha level ( 0.05 in the case of our study).

For its explanation, we can divide the method into two functions, the creation of the clusters, and the determination or not of statistical significance.

The method for the determination of statistical significance follows the next steps[57]:

1. Perform the statistical test on our data (the original sample).

2. Random partition of the samples: Randomly change the label of some of the samples.

3. Perform the statistical test in the random partition.

4. Repeat steps two and three a high number of times and calculate the histogram of the results, called the permutation distribution. 
5. Calculate the number of random partitions that resulted in a larger statistical test than that calculated on the original data.

6. Calculate the permutation $\rho$-value, from the permutation distribution.

The $\rho$-value is approximated using by the Monte Carlo method, by performing a large number of permutations and contrasting the statistics results obtained from these permeated values with those obtained from the original distribution. The pseudocode of the function implementing this issue is shown in function 1.This test ensures that the probability of falsely rejecting the null-hypothesis is equal to the defined $\alpha$-level.

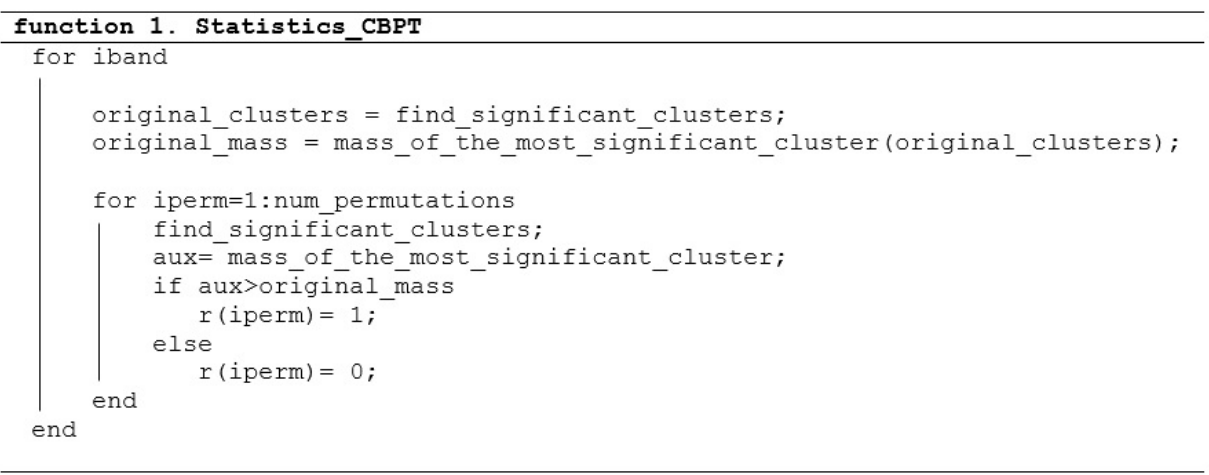

On his behalf, the creation of the clusters is carried out in two stages: check statistical adjacency and check spatial adjacency.

Statistical adjacency consists of determining whether the sample is statistically significant. That is, whether the two sources have statistically different levels of the characteristic being evaluated (the connectivity level in the case of our study). In this check, the sign of the statistical test is taken into account, discarding cases where the sign is not equal. If the sample passes the test, it is checked if it already belongs to an open cluster; otherwise, a new cluster is opened, and the sample is added to it.

The next step is the searching for significant neighbouring samples, the spatial adjacency. A fixed distance centred around the source is defined. All the significant samples with the same sign that fall within this volume are added to the cluster. This procedure is shown in Figure 2.7. 


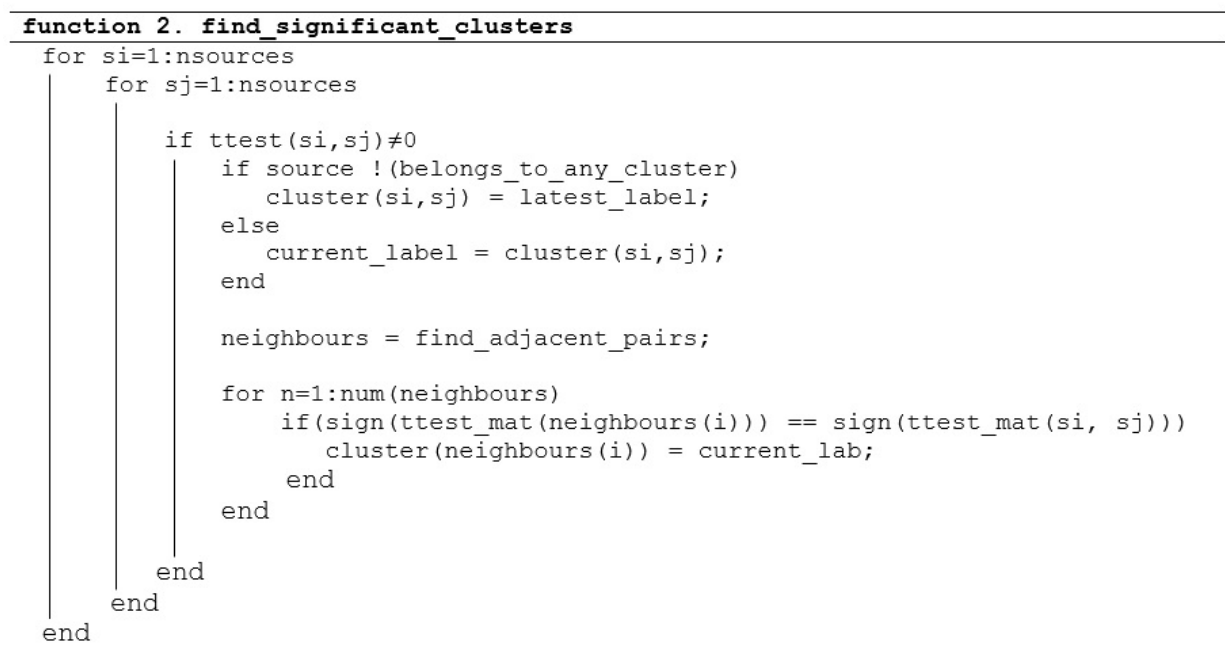

a)

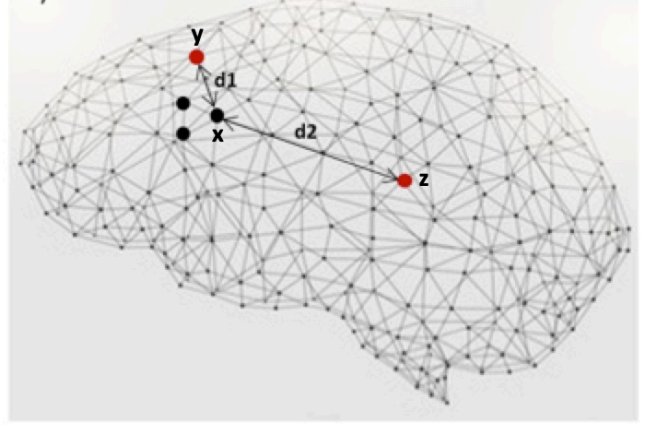

b)

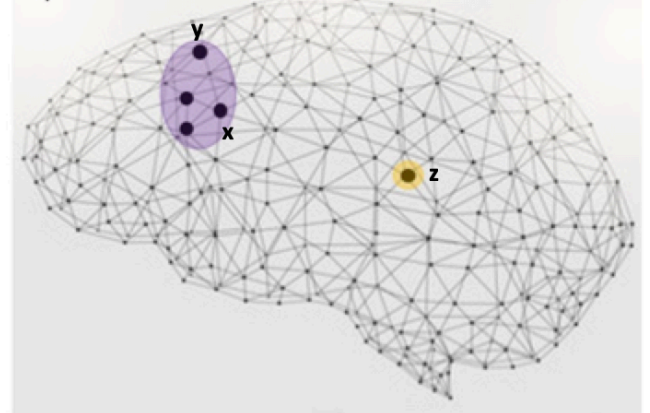

Figure 2.7: CBPT - Find adjacency pairs. a) The figure shows a scenario in which there is a cluster formed by three nodes (marked in purple). Point $x$ has just been added to this cluster. We proceed to look for neighbours to this point $x$. For it, the method looks for samples that are statistically significant and of the same sign. Suppose we find two samples that meet this condition, $y$ and $z$. b) $y$ point is within the determined distance, so it is added to the actual cluster (the purple cluster.) However $z$ is not in the neighbourhood, so it will be put in another cluster (represented in red) in futures iterations of the algorithm. 


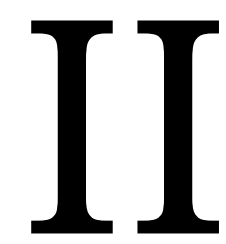

\section{DBS Mystery}

Nothing in this world is to be feared, only understood. Marie Curie

I am a brain, Watson. The rest of me is a mere appendix Arthur Conan Doyle 



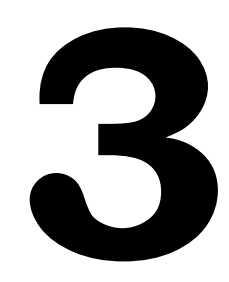

\section{DBS effects on cortico-cortical functional connectivity}

Despite the growing trend in DBS use, the exact mode of operation and the effects it produces on brain networks remain confused.

A higher level of understanding of the neurophysiological changes induced by $D B S$ would be an essential step for several reasons. Firstly, to gain insights into the therapeutic mechanisms of DBS. Secondly, based on which, the accuracy of DBS could be improved, perhaps avoiding or reducing adverse effects and monitoring treatment response. It will lead to a better understanding of the functioning of the brain, under different conditions.

This chapter presents a MEG study conducted in Parkinson's patients treated with deep brain stimulation. The aim is to study the effects that DBS induced on brain networks at the cortical level. 
The first hypotheses about the DBS action mechanism focused on whether the surgical target, the brain structure being stimulated, was excited or inhibited. $[58,59]$. Today the spotlight has changed, and the most recent studies focus on studying the effects of DBS on large-scale brain networks. A good part of the studies points out the importance of cortical networks, which may be involved in the benefits of stimulation. Therefore, the activity produced in the cerebral cortex during stimulation has become a piece of interest where MEG has turned a prominent tool in the study of DBS. As introduced before, MEG is a useful technique for studying cortical activity, being able to collect the neural activity directly with a very high temporal resolution and an excellent spatial resolution.

\subsection{Effects of deep brain stimulation}

Although DBS can override pathological activity, it introduces non-normal activity patterns [60]. Two aspects are of interest in brain electromagnetic activity, the neural oscillations and the neural synchronization. The former one provides information about the frequency at which a local group of neurons are working on, or oscillating while the second one finds the relation between the activity of different brain regions.

\section{Impacts in neural oscillations}

Inordinate levels of synchronised oscillatory activity in the beta band within the basal ganglia circuit, especially in the STN, has been found in PD patients [61-63] which has been linked to the clinical symptomatology [64]. It has been observed that this pathological activity also occurs in cortical areas $[62,65]$, and that it is related to the activity in the basal ganglia $[65,66]$. This abnormal activity is disrupted when stimulation is applied, modifying the activity in the BG-circuit and minimising the symptoms [67].

Combined LFP and MEG recordings have shown dysfunctional coupling between the basal ganglia and the cortex. Some studies have found that it is the activity of the cortical areas that directs the abnormal activity in the deep areas of the basal ganglia $[66,68]$. Similar studies also found an attenuation of beta-band both in the STN and over the sensorimotor cortex $[69,70]$, while others found an increase of the sensorimotor beta-band [71].

In [65] coherent activity was found in beta band in the ipsilateral sensorimotor and the premotor cortex, and in the alpha band at various locations in the ipsilateral temporal lobe. Others studies found that STN stimulation decreased spontaneous activity in the 3-20 Hz range over the right sensorimotor cortex [72]. In the same direction, other studies have reported suppression of alfa and beta bands of cortical areas has been linked to DBS at frequencies above $100 \mathrm{~Hz}$ [73], in the somatomotor area, linking this effects with rigidity [74].

Similar studies show that STN-DBS diminishes the oscillatory activity in the alpha and beta bands in the frontoparietal region while gamma activity is increased. The 
impact on clinical improvements is not clear. [75] found that the suppression in alfa and beta bands correlates positively with improvement in motor symptoms, while the increase in gamma does so negatively. However, the correlation with the symptomatology is not found in [76].

\section{Impacts in neural synchronization}

For its part, connectivity in cortico-subcortical connections has been addressed in some studies. STN-cortical coherence has been found in the low and high beta bands in the ipsilateral sensorimotor and the premotor cortex and in the alpha band at various locations in the ipsilateral temporal lobe [65]. This coherence is particularly prevalent at $20 \mathrm{~Hz}$, which is reduced with the administration of dopamine [77]. Moreover, the cerebral cortex has been found to drive the activity of STN in gamma [78] and in the beta band [70]. However, it also happens the other way around, being the phase of the STN and GPi which drives cortex in the $70-85 \mathrm{~Hz}$ band, which may be altered in the low dopamine state of PD [62]. In the same way, it has been suggested that the phase of the STN activity leads the cortex in frequency ranges $<30 \mathrm{~Hz}$. Unifying seems that the FC between cortical and subcortical structures seems to be bidirectional.

\section{Cortical Networks}

From literature, one can conclude that the exact mode of operation and effects with which DBS operates in the brain is a puzzle of which we have loose pieces, but not the complete picture. Recent studies suggest that cortical networks influence the clinical effects of DBS and vice versa. For this reason, studies are needed to delve into the cortical changes that occur with stimulation and its correlation with symptomatology [79]. To this end, the objective of this work is to study the effects produced in the functional connectivity at cortical level of the whole head, distinguishing between those that can be positive effects and adverse or spurious effects of DBS. To the best of our knowledge, this is the first study to delve into precisely this aspect.

Coordinated and integrated activity between brain areas is necessary for the processing of various brain functions, such as cognitive and motor [80]. The synchronization of neural activity among brain regions can reflect functional interactions between these areas. In this way, distributed neural populations could communicate by synchronizing their activity [81]. These interactions can be unveiled by measuring statistical interdependencies between physiological signals derived from different brain areas over a certain time window [45]. Relation in the oscillatory activity of different areas often implies a consistent relation between the phases of these oscillatory activities[39]. The assessment of resting-state functional connectivity has become an important tool in studying brain disease mechanisms since it has been seen that it can be disrupted by different neurological disorders [82, 83]. Specifically, in PD, it has been seen that functional connectivity at the cortical level is different than in healthy subject, changing with the advance of the disease, and correlating with the worsening of the symptomatology [82]. 


\begin{tabular}{|c|c|c|c|c|c|c|c|c|c|c|}
\hline \multirow{2}{*}{ Patient } & \multirow{2}{*}{ Gender } & \multirow{2}{*}{ Age } & \multirow{2}{*}{$\begin{array}{c}\text { PD } \\
\text { Subtype }\end{array}$} & \multirow{2}{*}{$\begin{array}{c}\text { Disease before } \\
\text { operation (yr) }\end{array}$} & \multirow{2}{*}{$\begin{array}{c}\text { Neurostimulator } \\
\text { implanted (months) }\end{array}$} & \multicolumn{2}{|c|}{ UPDRS } & \multirow{2}{*}{$\begin{array}{l}\text { LEDD } \\
(\mathrm{mg})\end{array}$} & \multirow{2}{*}{$\begin{array}{l}\text { DBS voltage } \\
\text { (right/left) }\end{array}$} & \multirow{2}{*}{$\begin{array}{l}\text { DBS stimulation type } \\
\text { right/left }\end{array}$} \\
\hline & & & & & & DBS-OFF & DBS-ON & & & \\
\hline$\# 1$ & $M$ & 36 & $\mathrm{R}$ & 8 & 5 & 71 & 26 & 210 & $3.0 / 3.0$ & mono/mono \\
\hline$\# 4$ & M & 49 & $\mathrm{R}$ & 25 & 9 & 25 & 16 & 1555 & $3.9 / 3.4$ & $\mathrm{bi} / \mathrm{bi}$ \\
\hline$\# 5$ & $M$ & 42 & $\mathrm{R}$ & 6 & 6 & 17 & 6 & 1663 & $5.9 / 3.6$ & $\mathrm{bi} / \mathrm{bi}$ \\
\hline \#6 & $\mathrm{F}$ & 64 & $\mathrm{R}$ & 25 & 8 & 40 & 18 & 651 & $3.4 / 1.4$ & $\mathrm{bi} / \mathrm{bi}$ \\
\hline$\# 9$ & $M$ & 47 & $\mathrm{R}$ & 8 & 6 & 45 & 6 & 458 & $2.3 / 2.5$ & mono/mono \\
\hline$\# 10$ & M & 71 & $\mathrm{R}$ & 8 & 6 & 27 & 12 & 819 & $3.7 / 3.7$ & mono/bi \\
\hline
\end{tabular}

Table 3.1: Clinical details of patients and stimulation parameters F:female; M:male;

$\mathrm{R}=$ rigid-akinetic; $\mathrm{T}=$ tremor; UPDRS=unified parkinson's disease rating scale; LEDD=levodopa equivalent daily dose; mono=monopolar; bi=bipolar; $(\mathrm{x} 2)=$ indicated stimulation with two monopolar contacts

In this study, we reconstruct, for the first time, source space whole-brain restingstate functional connectivity with MEG DBS-ON and DBS-OFF PD patients along with a group of control subjects in all the commonly studied frequency bands. Functional connectivity is evaluated through phase synchronization, more concretely by way of phase-locking value (PLV), which constitutes a fundamental mechanism for brain integration [81].

\subsection{Subjects}

The study sample consists of ten advanced PD patients and ten age-matched healthy control subjects (HC group). All participants signed informed consent before the study. The Ethics Committee approved the study of the Department of Neurology, Helsinki University Central Hospital. Their demographic data along with other relevant information is summarised in table 3.1.

PD patients went into surgery to have bilateral STN DBS (Kinetra or Activa PC Neurostimulators, Medtronic Inc., Minneapolis, MN, USA). This procedure was motivated by the severe adverse effects of motor fluctuations and dyskinesias that the patients suffered. The patients did not have clinical symptomatology of dementia, depression or psychosis. The diagnosis of PD was given 13.1 ( \pm 7.17) years before the STN-DBS procedure. The active contacts and stimulation parameters (frequency, voltage and pulse width) were individually set. MEG measurements were carried out 6.4 ( \pm 1.26$)$ months after the neurostimulator implantation. The patients used their normal medication during the measurements. The calculation of the levodopa equivalent daily dose (LEDD) was performed by the following expression: $100 \mathrm{mg}$ L-dopa $=130 \mathrm{mg}$ controlled release L-dopa $=70 \mathrm{mg}$ L-dopa + COMPT inhibitor $=$ $1 \mathrm{mg}$ pramipexole $=5 \mathrm{mg}$ ropinirole $[84]=4 \mathrm{mg}$ rotigotine $[85]$.

\section{Data acquisition}

MEG measurements were acquired with a 306-channel Elekta Neuromag ${ }^{\circledR}$ MEG device (Elekta Oy, Helsinki, Finland) in a magnetically shielded room. Subject's heads were digitised using a 3-D digitiser Fastrack Polemus ${ }^{\mathrm{TM}}$. Nasion and both preauricular fiducial points, in addition to a high number of points along the skull's surface 
were acquired per each subject. In addition, head position indication (HPI) coils were used so that the exact position of subject's head respect to the MEG helmet was continuously determined. The recordings were acquired using a sampling frequency of $1011 \mathrm{~Hz}$ and using an online anti-alias filter of [0.03-330] Hz.

Additionally, an MRI was recorded for each subject, since it is a necessary test before the surgery. By aggregating the MEG data with the corresponding MRI of each subject, a magnetic source imaging (MSI) study can be conducted, which allow the observation of the neural activity at the source level, achieving a good structural perspective [86].

\section{3. $M E G$}

\section{MEG data preprocessing}

Custom-made scripts in combination with Fieldtrip's [41] own and modified functions were employed in the preprocessing and source reconstruction process, described hereinafter.

Before any preprocessing, bad channels were discarded from further analysis. Then, the raw MEG data was denoised, discarding the signal's external components produced outside the brain through the Spatiotemporal space separation method (tSSS) using Maxfilter with parameters $\mathrm{L}_{\text {in }}=8, \mathrm{~L}_{\text {out }}=3$, tSSS correlation limit $=0.9$ and $\mathrm{tSSS}$ correlation window $=10$ seconds. Lower values for tSSS correlation limit has been demonstrated to be able to remove DBS artefacts from the signal [74]. However, I decided not to use values under 0.9 to avoid mixing brain signal components with disturbance waveforms as Elekta manual recommends. It was, therefore, necessary to use another type of technique to eliminate artefacts introduced by DBS, which is explained below.

Jump, muscle and ocular artefacts were automatically detected using Fieldtrip after which they were visually inspected and removed. Besides, an independent component analysis (ICA) procedure was employed to obtain the frequency components of the signal separately. All components were visually inspected to detect spectrum anomalies. In so doing, electrocardiographic components were removed.

As stated above, the stimulation introduces electromagnetic artefacts in the DBSON recordings, which were not entirely suppressed, employing a 0.9 correlation limit tSSS procedure. Those components containing DBS artefacts were into the following procedure, which pseudo-code is depicted in Algorithm1:

1. Visual inspection and labelling of each component. In this way, we differentiate the pure-DBS components (the ones that only contain DBS-ON artefacts), from the DBS-contaminated components (containing the signal of interest mixed along with DBS peaks).

2. Peaks present in pure-DBS components are located using a peak detection 


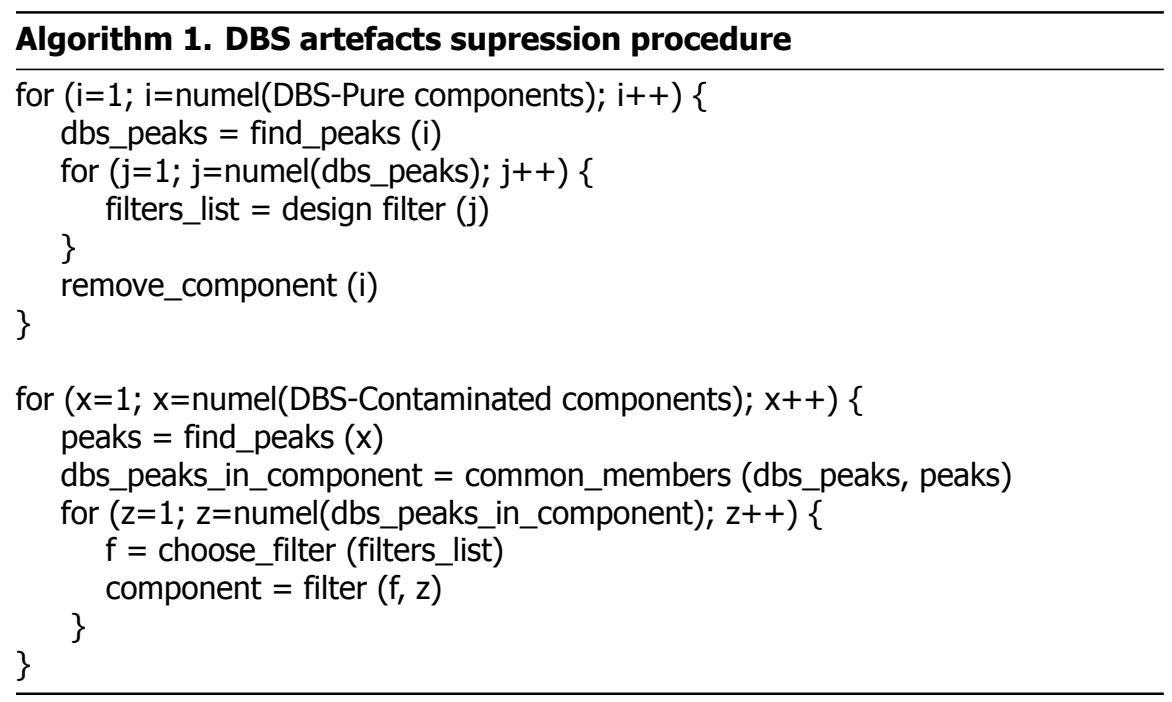

procedure. A $f s / 2$-order band-stop Butterworth filter is designed per each detected peak.

3. DBS-contaminated components followed the following steps: (i) Detection of the peaks present in the component: The peaks corresponding to the DBS artefacts are those that coincide in frequency-location with those extracted from the pure components, therefore are the peaks to eliminate. Note here that a contaminated component does not necessarily have as many artefacts as there are pure-DBS components. For this reason, this step has been carefully carried out manually. (ii) Filter the component with the corresponding previously designed filter(s).

4. Remove Pure-DBS components.

Finally, the data was segmented into 4 seconds of artefacts-free windows. Since the data is redundant after the Spatio-temporal filtering, only magnetometers data was used in the subsequent analysis.

\section{Source reconstruction}

The source space is composed of 2459 sources set in a homogeneous grid of $1 \mathrm{~cm}$ in the MNI template. This grid was then transformed into the subject space by warping the MRI of each subject into the MNI template. The labelling of the sources was done following the reduced Harvard-Oxford atlas [87]. In this process the sources that do not belong to any area of the atlas (such as for example the case of the sources located in the white matter) are eliminated, leaving for the subsequent analysis a total of 1489 sources. The forward model or leadfield was calculated by the three-shell Boundary Element Method (using brain-skull, skull-scalp and scalpair interfaces generated from the MRI) using OpenMEEG software[88]. 
Source reconstruction was performed in theta $(4-8 \mathrm{~Hz})$, alfa $(8-12 \mathrm{~Hz})$, lower-beta $(12-20 \mathrm{~Hz})$, upper beta $(20-30 \mathrm{~Hz})$ and gamma $(30-45 \mathrm{~Hz})$ bands with a Linearly Constrained Minimum Variance (LCMV) beamformer. The covariance matrix was averaged over all trials to compute the spatial filter's coefficients. Artefact-free epoch was two-pass filtered, to avoid phase distortion, into each band using band-pass filtering with an 1800 order finite impulse response (FIR) filter designed with Hamming window, and using 2 seconds of real data as padding to avoid edge effects within the boundaries of the data.

Source time series has been estimated with the computed leadfield and the beamformer filter, computed through the epoch-average covariance matrix.

\subsection{Analysis}

\section{Connectivity analysis}

Phase synchronization analysis employing PLV has been computed. See 2.3.2 for mathematical description.

\section{Statistical analysis}

Statistical analysis has been performed using Cluster-based permutation test (CBPT), which avoid the multiple comparison problem (MCP). See 2.4.2 for algorithmcal description.

Additionally, we perform a second level of statistical correction applying stepwise Bonferroni method to obtained clusters, additionally correcting them for MCP. This method adapts the alpha value from which the cluster is considered significant as a function the order occupied by the cluster. The order of the clusters is defined taking their mass in a decreasing way. The adjustment of the alpha-level is set as $p_{i}=$ $0.05 / i$. Thus, the first cluster is considered significant if its mass is higher than $95 \%$ of the randomized clusters $(p<0.05)$. Subsequently, the second cluster is considered significative if its mass is higher than $97.5 \%$ of the randomized clusters $(p<0.05 / 2)$, an so on. [89]. Only the survival clusters of this strict statistical procedure were used in the subsequent analysis; In this way we can be absolutely sure of the significance of the results obtained.

\section{Analysis Paradigm}

This study aims to delve deeper into the effects that DBS has on functional connectivity at the whole-brain resting-state functional connectivity level. For this purpose, the connectivity levels of the patients, both in the DBS-OFF and DBS-ON states and of the control subjects are calculated. The simple comparison of FC values between DBS-OFF and DBS-ON states would disclose differences induced by stimulation. However, this analysis does not provide enough precision to know in which direction these differences occur. This work aims to investigate all the effects produced by DBS, differentiating those that are beneficial for the pathology from those that could be considered side effects. To this end, here not only the study 
of the differences between ON and OFF conditions is carried out but also patients are contrasted against the healthy controls to gain insight into the differences between them in terms of connectivity at a cortico-cortical level. This forms a baseline for interpreting the direction of the effects introduced by DBS. Thus, the following statistical comparisons are computed:

DBS-OFF vs DBS-ON . By comparing OFF and ON DBS conditions, the differences in connectivity between areas as a consequence of stimulation are obtained.

DBS-OFF vs CTRL . The comparison of the patients in the absence of stimulation with the healthy control subjects reveals which connections between areas differ significantly in their connectivity, with respect with the values that are presented in the absence of pathology. That is, the connections between areas that are affected in their level of FC as a consequence of Parkinson's disease. These will be therefore the links susceptible to being modified by the stimulation.

Having the links which are modified both by DBS and by the pathology, the determination of all possible DBS outcomes is performed as explained hereinbelow and depicted in figure 3.1:

DBS-OFF vs DBS-ON $\in$ DBS-OFF vs CTRL . This comparison reveals the significant links due to stimulation that are also among the links affected by the pathology, and on which DBS has had some effect. That is to say, the subset of links that had to be modified, given their significant difference with the HC group, which are actually modified by the DBS.

In order to know if this effect acts only in the direction of treating the pathological symptomatology of Parkinson's, or (also) induce adverse effects, it is necessary to check if the connectivity values during DBS-ON approach or move away from the connectivity values presented in the HC group. Tree possibilities are given, it will be determined that:

- DBS fixes the pathological FC presented in PD if the values of connectivity of conditions DBS-ON and CTRL are equal. This only would happen if DBS fixes all the links whose connectivity was significant in DBS-OFF vs HC.

- DBS improves the connectivity levels of the damaged links if the DBS-ON state constitutes an intermediate state between DBS-OFF and HC.

- DBS worsens the value of connectivity in the links of interest if DBS-OFF state is an intermediate state between DBS-ON and HC.

DBS-OFF vs DBS-ON $\notin$ DBS-OFF vs CTRL . Analogously, we can say that significant links due to stimulation (DBS-OFF vs DBS-ON) which in turn are not significant links induced by the pathology (DBS-OFF vs CTRL) are those links in which the stimulation has modified the connectivity but that it should not have done it. 
These induced change in the connectivity of the affected links it will be considered as adverse effects.

For the shake of better understanding and a complete description, truth-table 3.2 summarises in a logical way, all possible effects that DBS could induce. 


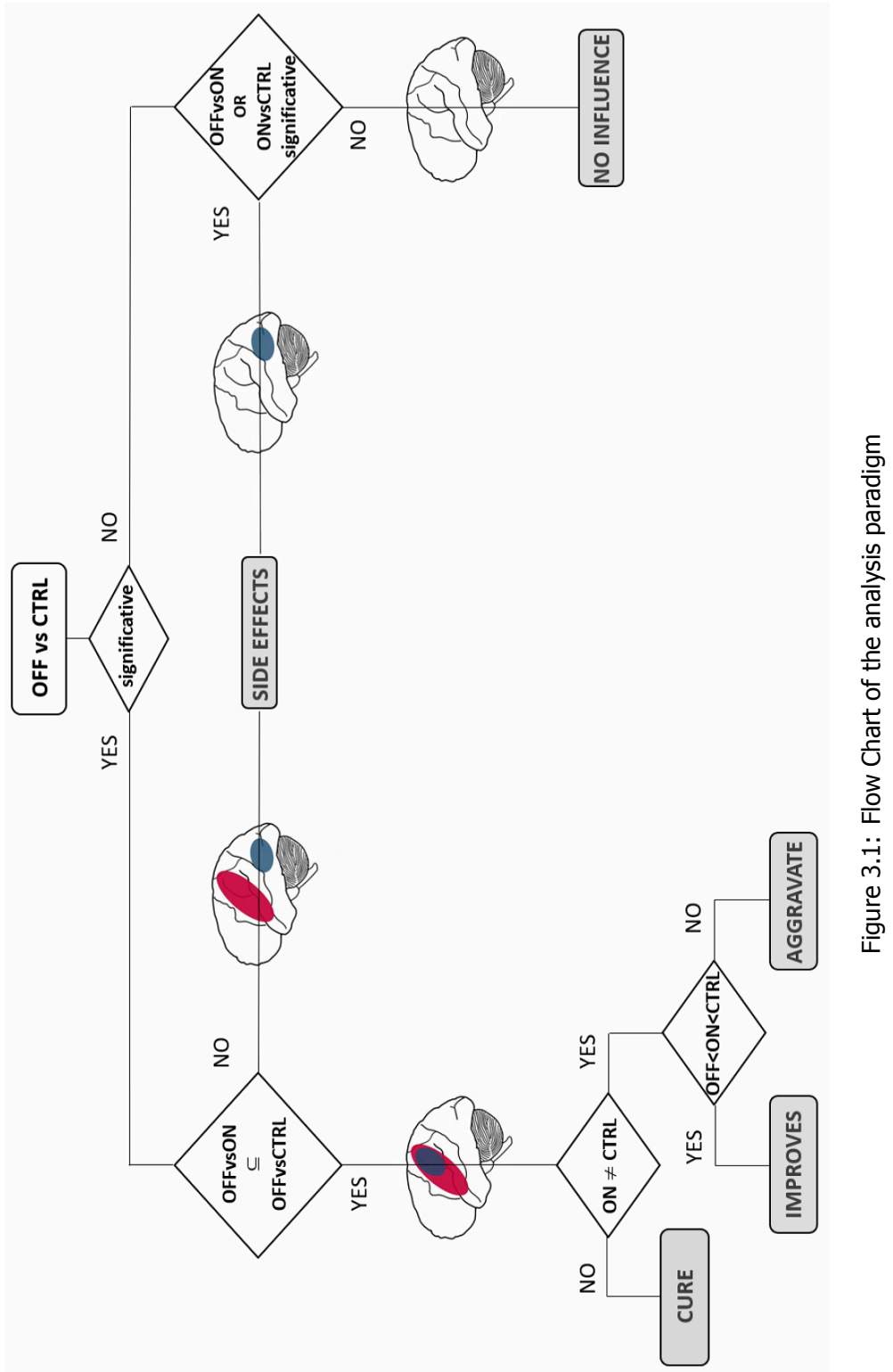




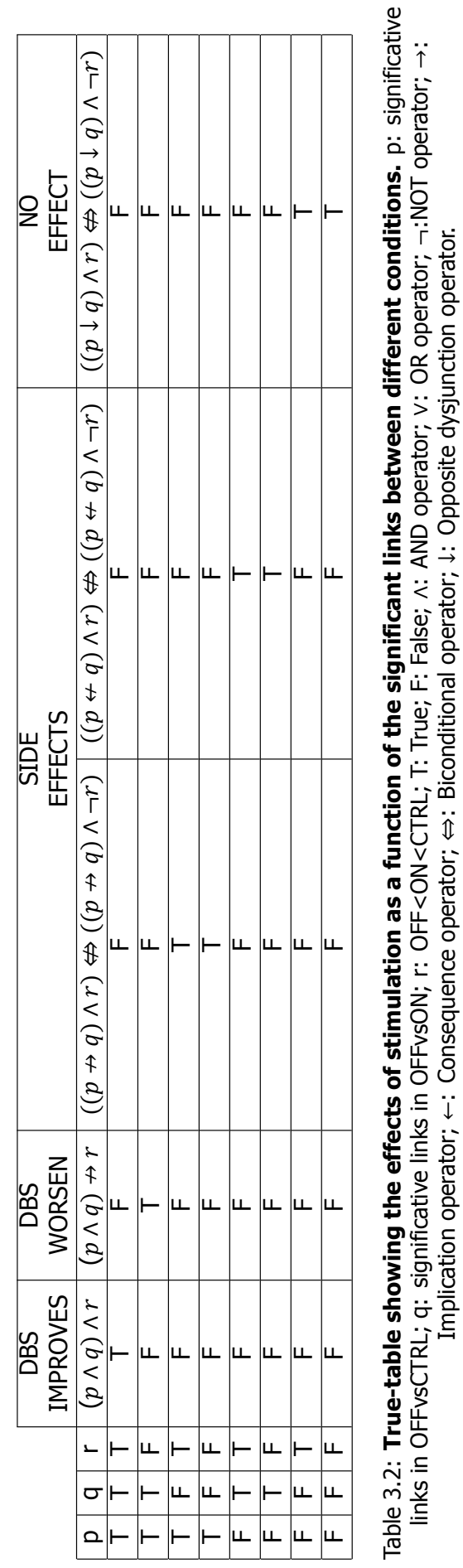




\subsection{Results}

\section{DBS effects on resting state cortical FC}

In relation to the connectivity for each link between areas, deep brain stimulation can theoretically produce five possible outcomes, as shown in Figure 3.1: 1)To fix, 2) to improve or 3)to worsen the connectivity level of the link; Matching, bringing them closer to or away from the connectivity levels present in the $\mathrm{HC}$ group respectively. DBS could also 4) produce adverse effects or the other way around 5) have no incidence on the connectivity levels of the link.

In practice, results have only been found in cases 2,4 and 5 . Therefore, no cases have been found in which the connectivity of the affected links has been wholly repaired (case 1), nor cases in which the connectivity levels of an affected link are worsened (case 3). Results of the analysis are depicted in figures [3.2 - 3.5] and are explained below.

\section{Theta}

In the theta band, seven links show a significative effect in improving levels of connectivity under stimulation (Figure 3.2). These links connect the right temporoocipital areas with right frontal areas, right precental gyrus and the left paracingulate. Adverse effects have been found in links between right temporal and right frontal areas. There is also an aberrant level of induced connectivity within the right temporal area between antero-posterior STG and posterior STG areas.

\section{Alpha}

No significant results have been found in the alpha band.

\section{Lower-Beta}

Beta band, both in lower and upper beta, is where the highest effect of stimulation is concentrated (Figure 3.3). The adverse effects seem to affect only the right hemisphere, in links that connect frontal and temporal and temporal with temporalmedial areas. Here it is interesting to see how one of the affected links involve amygdala and hippocampus [90], both related to depression. This could be related to adverse effects related to the depression that DBS has been shown to induce [91].

Nevertheless, the beneficial effects of stimulation in this band are substantial. Up to 66 links improve FC levels during DBS-ON. The vast majority of beneficial effects are found in links that connect frontal with posterior regions, both between intra- and inter- hemispherical areas, among which is the motor cortex in both hemispheres. Especially interesting is the case of the lingual gyrus and calcarine cortex areas of the right hemisphere, which appear as switches involved in a large number of connections.

Additionally, in the left hemisphere can be found a small network of links connecting temporal-medial, parietal and occipital areas, including the right amygdala. 


\section{Upper-Beta}

The adverse effects of the stimulation in this band compromise the same regions involved in the lower-beta case, with small differences (Figure 3.4). However, the positive effects are different, finding fewer links and fewer regions involved in them. Inter-hemispheric improved links are found between left parietal and right frontal regions and in left occipital areas connected the right motor cortex. The rest of the improved connections are in the left hemisphere. Links are found in all the left regions, but two sub-networks can be distinguished. The first is a medial-posterior network that connects links of the temporal-medial, parietal and occipital regions. The other subnet connects posterior zones with the frontal and somatosensory regions.

\section{Gamma}

Only adverse effects are found in the gamma band (Figure 3.5). These affect a total of nine links, three of which connect right frontal pole with all the areas within the temporal medial region, including the amygdala. Others connect the right parahippocampal and the left frontal medial cortex. The rest of it consists of connections of the right hemisphere between areas of temporal and frontal regions.

\section{Correlation Analyses}

Correlation tests between DBS effects, both in the links correctly and in the incorrectly modified links, with corresponding changes in UPDRS scores and LEDD have been performed. The significative correlations and links are shown in table 3.3. The significative correlations and links are shown in table 3.3. 

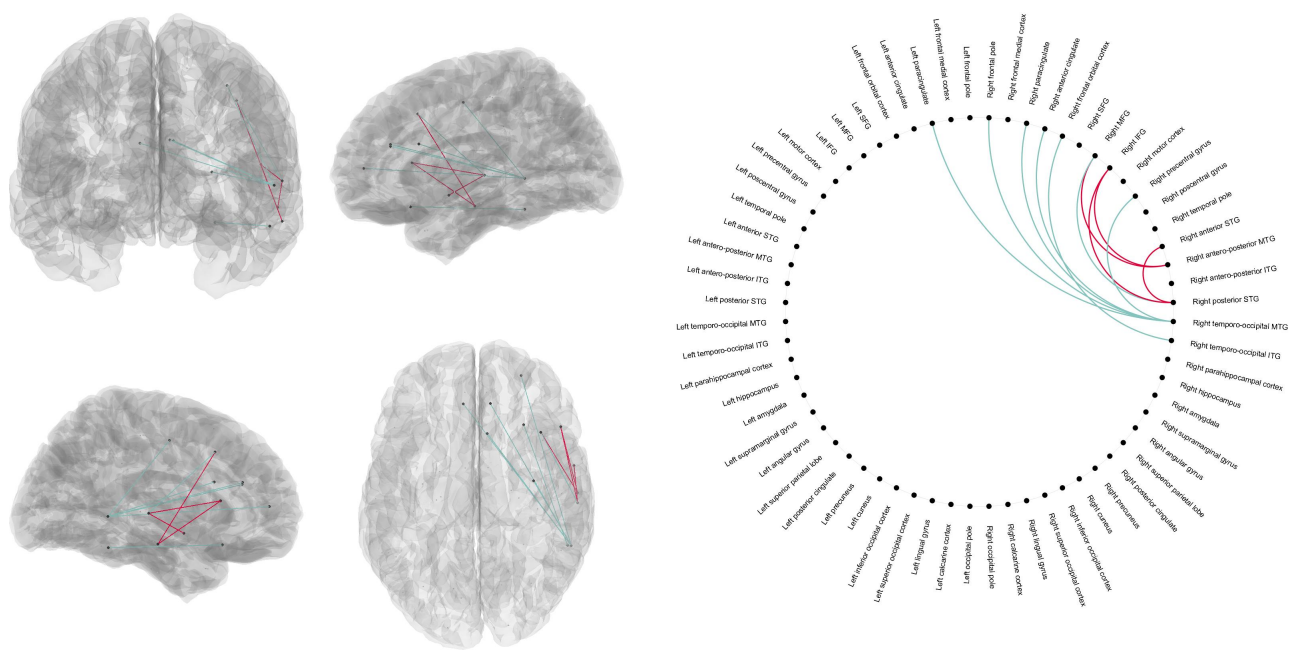

Figure 3.2: Changes induced by DBS in the resting state cortical FC in Theta band
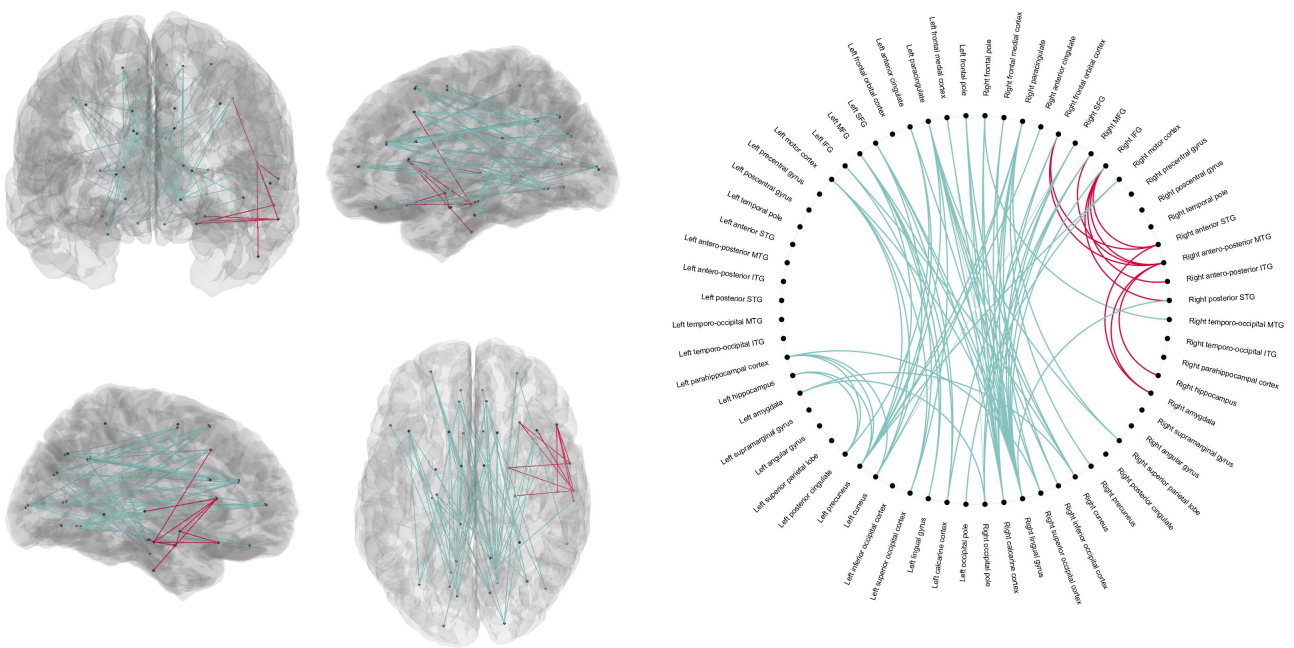

Figure 3.3: Changes induced by DBS in the resting state cortical FC in Lower-Beta band 

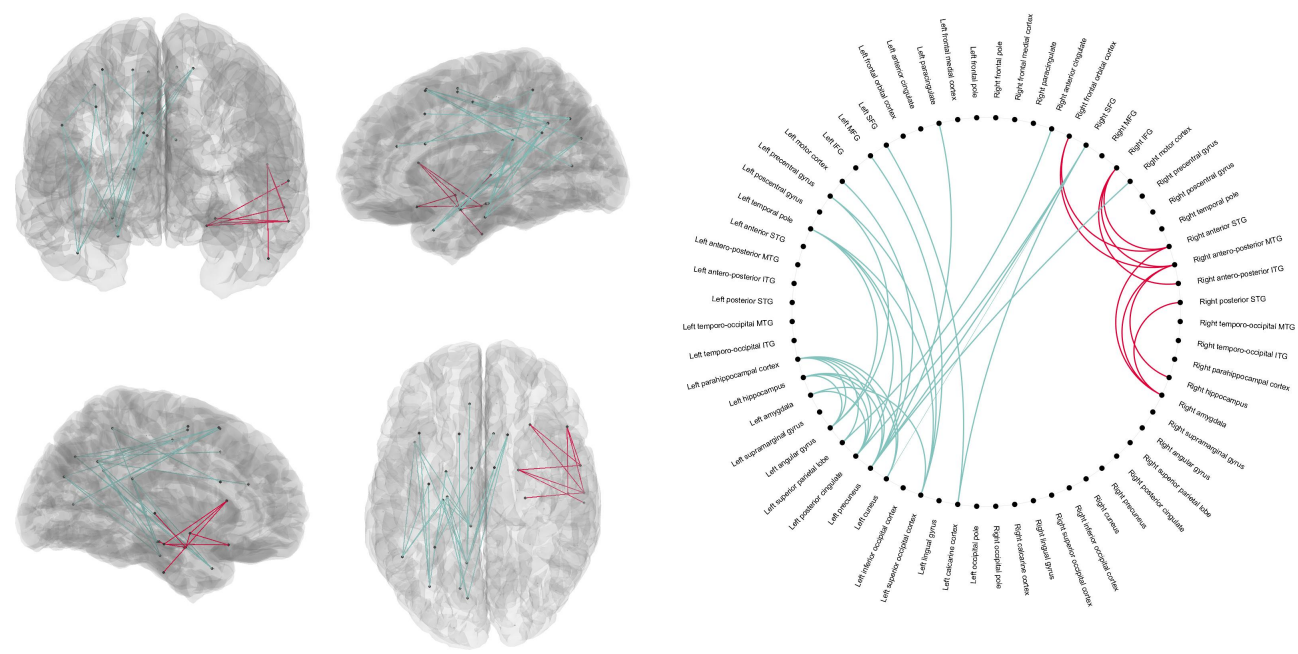

Figure 3.4: Changes induced by DBS in the resting state cortical FC in Upper-Beta band
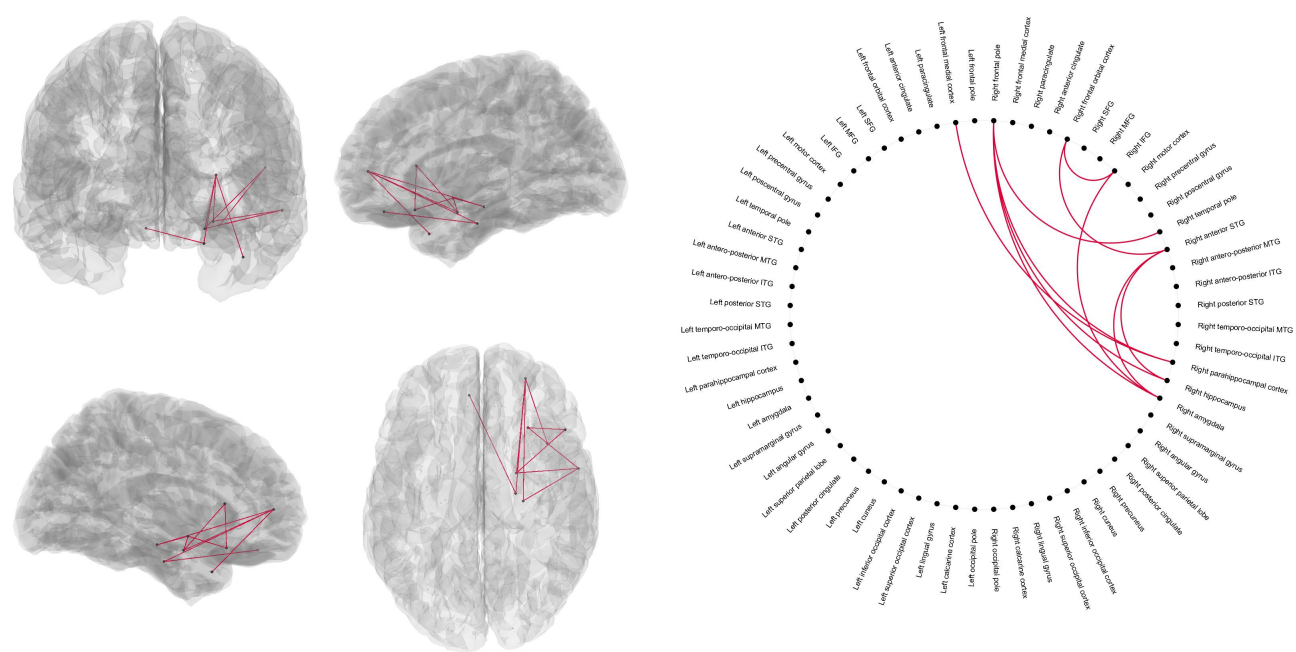

Figure 3.5: Changes induced by DBS in the resting state cortical FC in Gamma band 


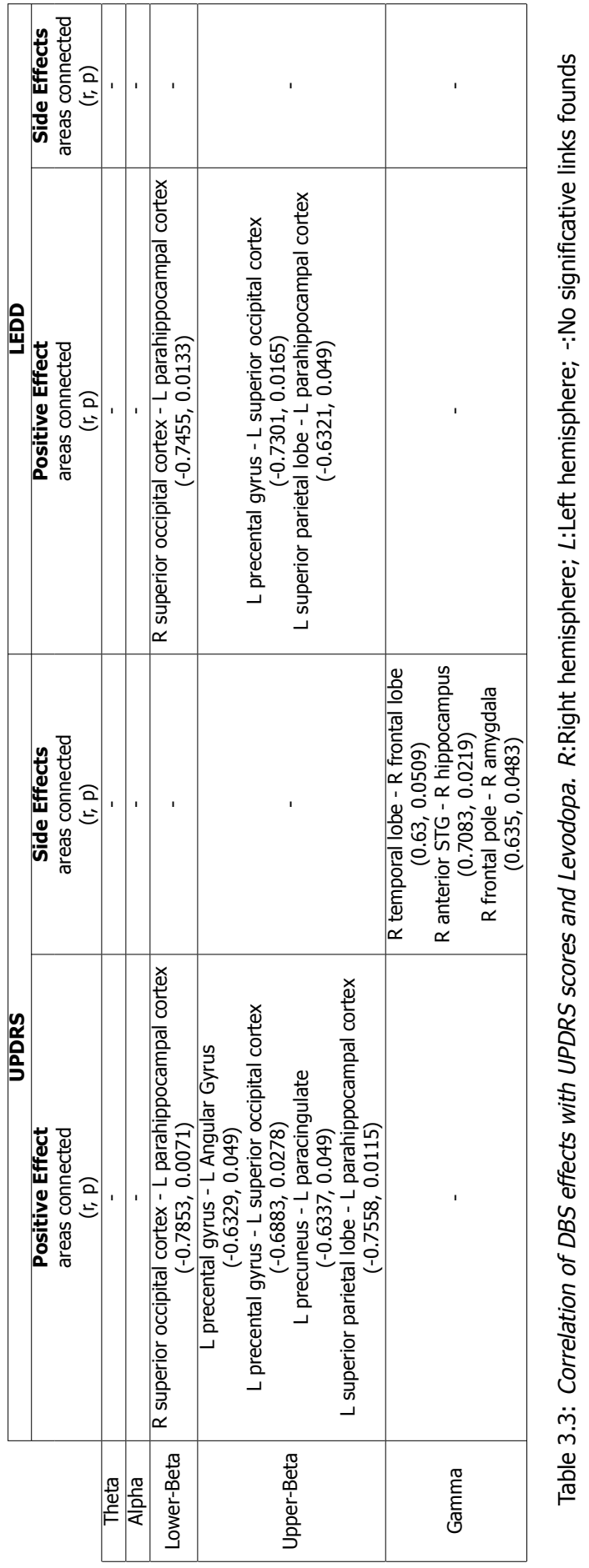




\subsection{Conclusions}

Two main contributions can be drawn from this study:

First, the links to be modified have been differentiated from those that the stimulation should not affect. The links to modify are those that present significantly different connectivity values between the patient in DBS OFF condition and the HC group. For its part, the links on which the DBS should not act are those in which these differences do not exist, always speaking from the point of view of functional connectivity.

Secondly, the effects that the stimulation produces on these links have been determined, differentiating between beneficial and side effects. Beneficial effects have been considered those that are given in links that had to be modified and that approximate the connectivity values to those present in $\mathrm{HC}$. While if the connectivity of a link that was not to be modified is altered as a result of stimulation, this will be considered adverse effects.

From the results, the following conclusions can be derived:

Affected areas . The effects of DBS are not restricted to the motor and sensorimotor areas but include others previously suggested in the literature, such as the frontal and parietal regions. But in addition, the results of this study show that these effects also occur in other regions unexplored in previous literature.

Positive and Negative effects . One of the main conclusions of this study is the finding of patterns of alterations common in frontal and temporal regions of the right hemisphere in all the frequency bands studied. The results show that the adverse effects affect the same regions in all frequency bands, involving the same or nearby areas.

While the links correctly modified by DBS, occur in different areas forming diverse networks in each of the frequency bands studied. Interestingly, most of the links that improve connectivity are in the beta band. This band has been widely studied in the literature given its role in PD. Although most of these works study spectral properties, in this study, we show how the beta band seems to play an essential role in FC as well.

As a summary on the effects introduced by stimulation, it can be concluded that the beneficial effects of DBS are widely distributed throughout the cerebral cortex and are dependent on the frequency band, while the adverse effects are located in specific regions of the right hemisphere and not being frequency-dependent.

Nature of the effects . When compared to the DBS-OFF condition, DBS-ON exhibits an increased FC in the affected links, while it shows a decreased FC when compared with $\mathrm{HC}$. This suggests that DBS-ON as an intermediate state between DBS-OFF and HC. These results seem to be consistent with previous studies that 
suggest FC in PD patients is diminished compared to controls, not having inference in the results the dopaminergic effect of levodopa [82]. In that study, the connectivity is calculated only between some cortical regions. Here we have studied whole-brain FC, and the results are along the same lines in all the regions involved.

To the best of our knowledge, this is the first work studying whole-brain FC alteration due to DBS and determining the links that should and should not be modified by the stimulation. The results found to shed light on what we wanted to call "DBS Mistery", in other words, what changes take places in the brain of a PD patient during stimulation. But, aside from advancing to unravel this mystery, we believe the results could be used in the design and development of connectivity-based DBS strategies, which may improve clinical response, trying to enhance the benefits and avoid adverse effects, perhaps in an intelligent closed-loop system. 


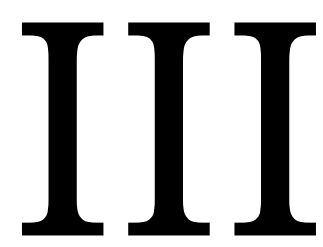

\section{Closed-loop DBS}

I am always doing that which I cannot do, in order that I may learn how to do it.

Pablo Picasso

You don't understand anything until you learn it more than one way. Marvin Minsky 



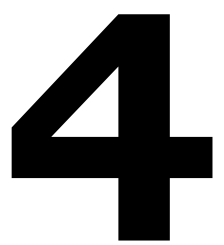

\section{Closed-loop Deep Brain Stimulation}

Closed-loop DBS systems represent an advance in the treatment of movement disorders. These systems will allow personalized treatment in realtime, avoiding part of the adverse effects of continuous stimulation.

These methods have gained importance in the last five years. They are still proposals from research groups, not implemented in the actual devices. However, they undoubtedly form the basis of the new generation of medical devices. In the future, all medical devices will operate in closed-loop mode, on-demand. 
arkinson's disease encompasses different subtypes. In this thesis, I work with patients suffering from benign tremulous parkinsonism. The symptomatology present in these patients includes: 1) A prominent RT, being this the main symptom; 2) symptoms not related to tremor remain mild; 3) majority absence of gait disorder; 4) absence of disability apart from tremor [92].

RT makes the patient transit between different movement states: non-tremorous resting state $(N T)$, in which the patient does not experience symptomatology; the tremor state $(T)$ itself, in which the patient experiences RT, and a third state called Tremor Onset (TO), which is supposed to hold the key to understanding the transition between $N T$ and $T$. Similar dynamical behaviour of the brain, transitioning between different states, can be found in other diseases such as epilepsy, which are known as dynamical diseases [93].

As previously introduced, to alleviate these symptoms, the first option is usually a pharmacological treatment. However, these patients have a tremor with high resistance to medication, even at the highest tolerable doses of levodopa [92]. In addition, the use of levodopa leads to dyskinesias (LID), in which the patient suffers from involuntary movements that may ultimately be worse than the original PD's symptomatology. DBS constitutes a line of treatment in such cases. Treatment with DBS can be administered in conjunction with levodopa if necessary, for the rest of the symptoms, if present, including bradykinesia and rigidity.

In the context of cardiac illnesses, pacemakers can adapt the stimulation to perform event-response in real-time. However, current neurostimulators work in an open-loop way, providing stimulation uninterruptedly since the moment of its implantation. As a consequence, there is a reduction in efficiency over time. Moreover, several adverse effects, related to this continuous stimulation, have been reported in the literature, including paresthesia, cognitive or psychiatric dysfunction, and even an increased risk of suicide $[94,95]$.

Real-time adaptive (closed-loop) DBS systems represent a better strategy, in which the IMD stimulates only when necessary, on-demand [96-99], thereby reducing the adverse effects. These systems will sense continuously a feedback signal, through which to infer a biomarker, that correlates with the patient's symptomatology. The implementation of such a strategy in PD requires, therefore, the knowledge of what features of the STN activity change when (or ideally, shortly before) the clinical symptoms appear.

Disentangling the behaviour of the STN in the states mentioned above (NT, TO and $T$ ) is the key to understanding the nature of the tremor. Furthermore, studying the temporal dynamics of such activity could be useful as well in designing better DBS treatments, even tailoring them to the individual concerned, as a further step towards personalised medical care. However, this task is arduous, since it requires recordings that capture the neural activity of patients while transitioning from $N T$ to $T$, naturally and spontaneously, in the absence of medication. Furthermore, local 
field potentials recorded from the STN (STN-LFP) in humans are generally acquired in the peri-operative period, after the surgical implantation of the electrodes, but prior to the final internalisation of the neurostimulator, and are not usually accessible afterwards. These two restrictions make this kind of recordings exceptional.

In this thesis, four of them from our dataset are analysed, which fulfils these properties. The original dataset is composed of 10 patients (and 33 files), and only four of them capture the neural activity of patients while transitioning from NT to $T$, naturally and spontaneously, in the absence of medication. This ratio (4 records out of 33) reflects the difficulty in obtaining recordings such as those used in this research. Figure 4.1 illustrates the three mentioned dynamical states - which will be called states hereinafter.

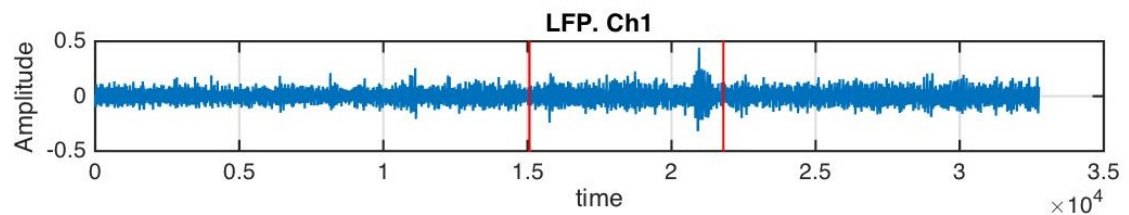

LFP. Ch2

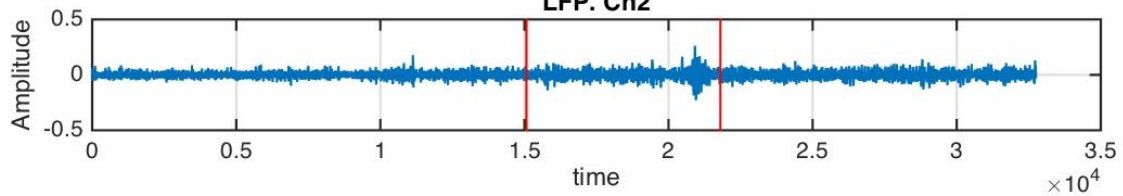

LFP. Ch3

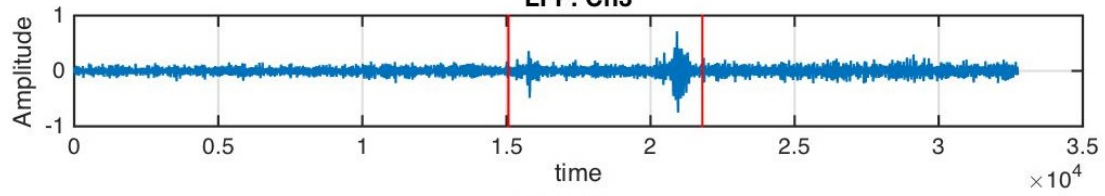

EMG Extensor

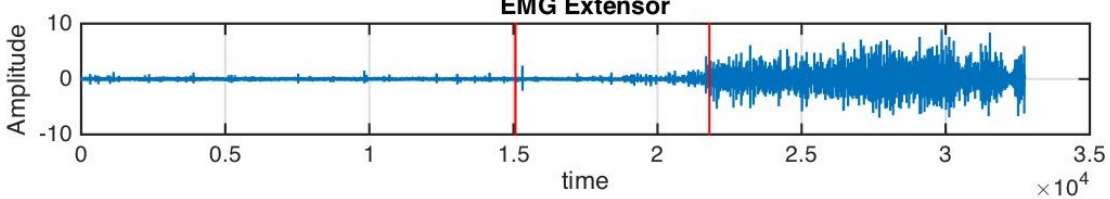

Figure 4.1: The three top panels represent the LFP signals collected through contacts 1 to 3 . The bottom one is the flexor electromyographic signal simultaneously recorded (not used in this study). Vertical red lines separate the states of atremorous (NT), tremor onset (TO) and tremor $(T)$. In the EMG signal, the temporal point in which tremor start is evident, but not quite so in the LFP signals. We will investigate later if synchronization measures are able to distinguish these three states.

Closed-loop neurostimulation is an umbrella term which encompasses different advanced DBS strategies, that apply various approaches to treat the symptoms. We can find the following families of closed-loop DBS strategies [100]:

- Adaptative DBS. These methods propose a real-time adaptation of HFS parameters (the frequency, duration and amplitude of a square-wave pulse train) 
which are currently determined by a clinician during the visit of the patient to the hospital every 3-12 months.

- Demand-driven DBS. These strategies are based on detecting the "fingerprints" of pathological states and the triggering the HFS as a result.

In my opinion, the combination of adaptative and demand-driven DBS approximations would provide a complete solution for an autonomous and intelligent DBS system, able to adapt the stimulation parameters by itself, and also capable of start-up and shut down itself as required by the changing dynamics of the STN in real-time.

- Delayed DBS. These strategies consist of providing stimulation in a timedelayed manner, with the added possibility of doing it in different areas using several electrodes. The objective is to concentrate a beam of out-of-phase sinusoidal signals in the target area.

- DBS based on proportional, derivative and integral feedback. These methods propose to design a stimulation signal following the LFP signal sensed in real-time. This signal can be designed proportionally to the LFP activity or regarding integral or derivative LFP.

- Optimal control strategies. These techniques base the control of the stimulation policy on finding the minimum of a defined cost function. This cost function will be adjusted to the DBS objectives, such as beta-band oscillation reduction or neuronal desynchronization.

The studies carried out in this thesis present proposals within the demand-driven DBS strategies. Focusing on this area, we compare the results obtained with those other studies in this area that provide the level of accuracy of their systems. Wu et al. [101] proposes a system using a radial basis function neural network based on particle swarm optimization trained with STN-LFP signal. The system reaches an $89.91 \%$ of accuracy. In [102], the authors examine several STN-LFP characteristics of both time and frequency domain, and characteristics based on information theory. After a feature selection process, they train a feed-forward neural network classifier, obtaining an $86 \%$ of accuracy. Basu et al. [103] proposed a system combining EMG and LFP signals making use of both spectral and non-linear properties of the signals, obtaining an $80.2 \%$ of accuracy. In [104], the authors study the spectral characteristics of the LFP signal, classifying it in the different states of tremor employing hidden Markov models. They obtained an accuracy level of $84 \%$.

Systems that make use of both the muscle and the subthalamic signals have the disadvantage of needing an external device that senses the EMG signal for its operation. In this sense, systems that make use only of the STN-LFP signal are more functional, as they could be included in the existing DBS montage. In my opinion, this is a crucial characteristic to take into account. 


\subsection{Dataset}

The data used in this thesis consists of a set of recordings from parkinsonian patients diagnosed with benign tremulous PD, who underwent surgery for the implantation of a neurostimulator (DBS treatment) at the John Radcliffe Hospital in Oxford, UK. The successful implantation of the DBS electrode lead within the STN was verified with postoperative magnetic resonance.

Neurophysiological data was acquired by employing a Medtronic DBS Lead Model 3387, which contains four electrodes spaced $1.5 \mathrm{~mm}$ apart. This allows for three different contact pair (electrode) configurations $(0+1,1+2$, and $2+3)$ to be simultaneously recorded in a bipolar fashion with one contact used as reference. Thus, each record consists of three channels of LFP, collected through the electrodes located in the basal ganglia, specifically the STN. As seen before, LFPs capture the summarised electrical activity of the neuron population between each contact. Recordings were performed during a peri-operative observation period in which the depth electrodes were already implanted but were not stimulating. As a result, data was accessible for recording. Note that the recordings have been obtained under DBS-OFF condition. In this regard, the validity of the proposed systems in recognizing the patient's clinical states in a DBS-ON context could be questioned. Nevertheless, as explained in chapter 9, this will not be a problem in the new generation of neurostimulation devices, in which closed-loop strategies are framed.

Additionally, simultaneously to the LFP record, electromyography (EMG) signal was acquired in order to label the data into tremorous and atremorous sections. The EMG records were taken from the extensor with the arm contralateral to the neurostimulator implantation side.

All patients gave their informed consent for inclusion before they participated in the study. The study was conducted following the Declaration of Helsinki, and the protocol was approved by the local research ethics committee of the Oxfordshire Health Authority (REC reference number 08/H0604158).

\section{Dataset subsets}

The recordings were obtained under different movement conditions. In this way, the files can be classified into two sets:

- Non-transition files. These are files that contain a single motion state. During registration, the patient was either experiencing tremor at rest or showed no symptoms. So, there is only one label for each file, which can be $T$ (the file contents tremor signal) or NT (the file contents signal generated during an atremorous state). These files will be used in the works presented in chapters 5 and 6.

- Transition files. The recordings capture the neural activity of patients while transitioning from NT to $\mathrm{T}$, in a natural and spontaneous way, in the absence of medication. Thus, the file contains samples of neural activity sorted as NT 
$\rightarrow T O \rightarrow T$. These files will be used in the works presented in chapters 7 and 8.

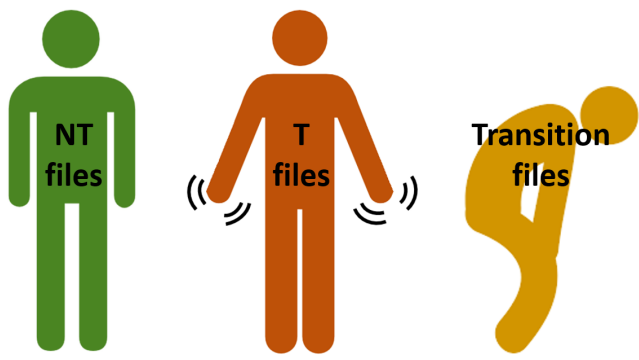

Figure 4.2: File types depending on their content. Tremor (T) files contain neuronal signal samples recorded in states in which the patient suffers from resting tremor. Atremorous (NT) files contain neuronal signal samples recorded in states in which the patient is not experiencing symptomatology. Transition files contain neuronal signal samples recorded in states in which the patient begins not trembling and at a given moment begins to experience tremor at rest.

\section{Data Labelling}

Each recording holds a single tremor-onset event. A clinical specialist in movement disorders marked the time in which the tremor starts. Tremor onset was calculated based on the amplitude of filtered and rectified EMG signal, following the same procedure used previously with this data [102]. The magnitude of the EMG time series value was checked against a threshold of 3 times the mean of the EMG amplitude in the first $5 s$ of the recording (which contain atremorous data). If a peak of high tremor frequency activity is detected at any point in time, the average of the following $5 \mathrm{~s}$ (time enough to cover any period of tremor-onset) of data is calculated to confirm the tremor-onset detection. Specifying a threshold would also detect peaks of tremor activity, however short magnitude spikes may trigger an incorrect detection.

A single time of tremor-onset was calculated for each recording. This mark, together with the one determined by the clinical specialist divide the recording into the three tremor states of which it is composed: NT(atremorous state), TO(tremoronset state) and $\mathrm{T}$ (tremor state). Following figures represent the tremor-onset detection into the EMG signals (left side) and the time-frequency representation of the EMG channel in the tremor band [3-7] Hz for each transition file (right side). As can be seen, in all cases the tremor-onset state constitutes an intermediate power state between the atremorous and the tremor states. 

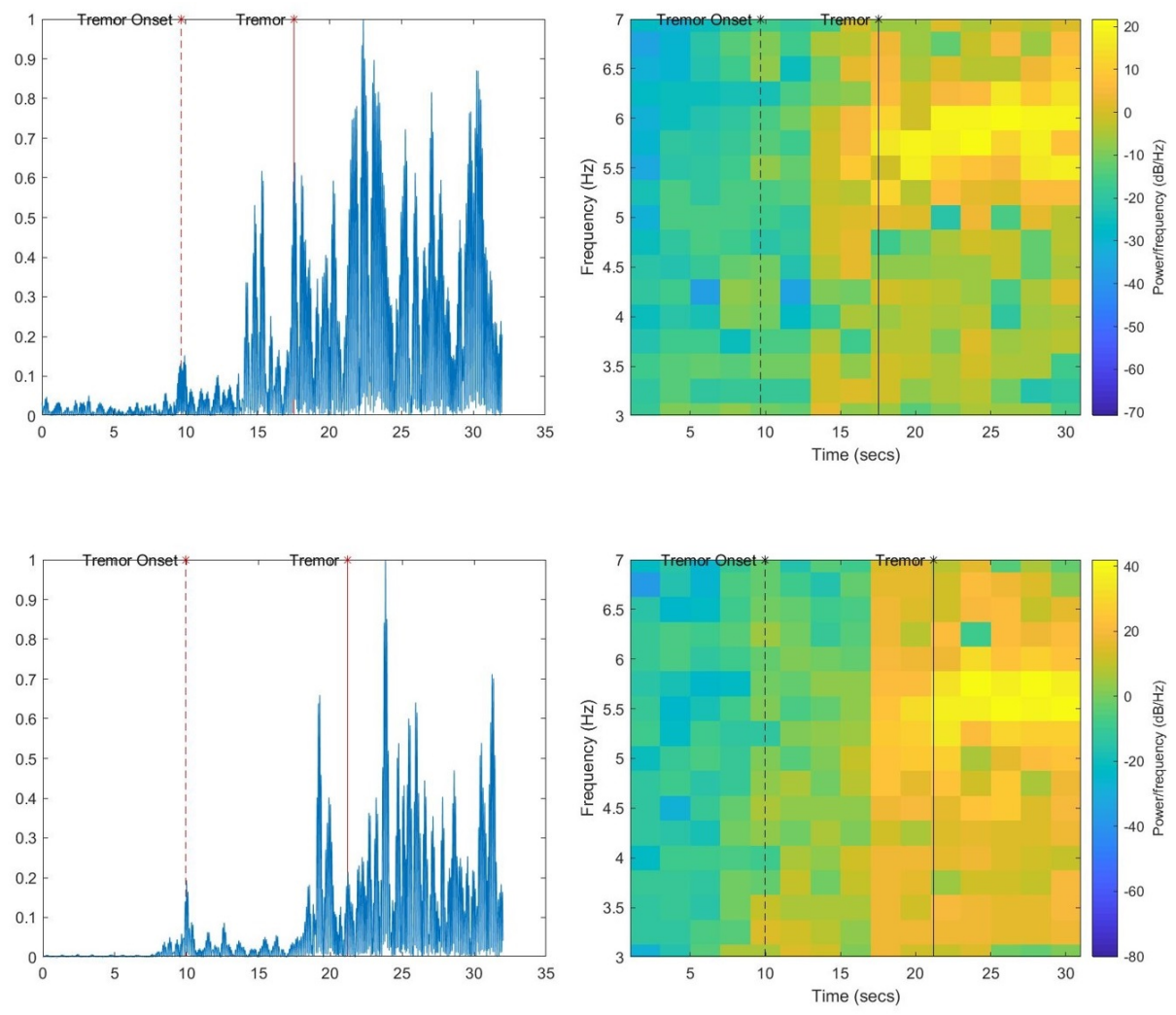

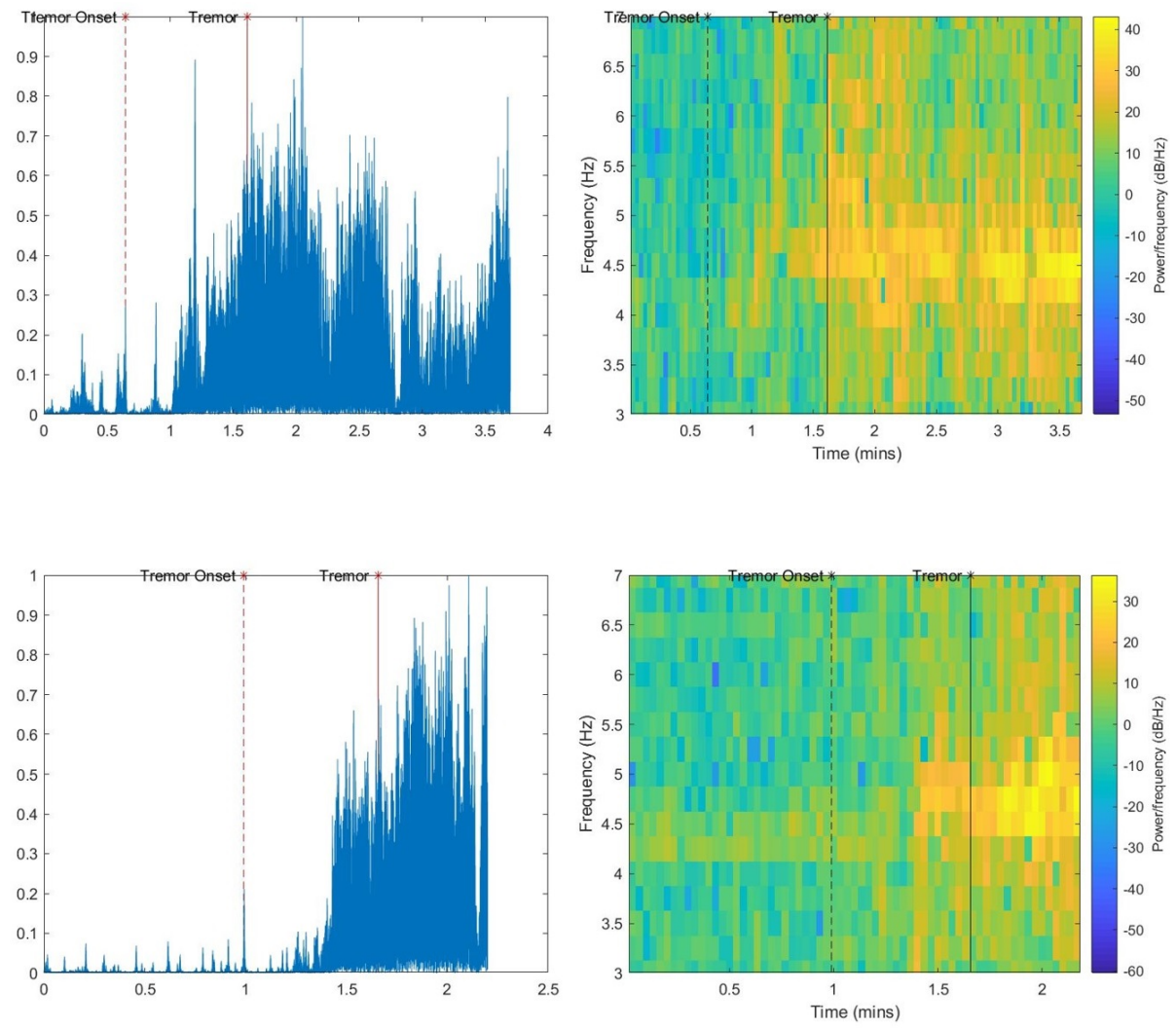


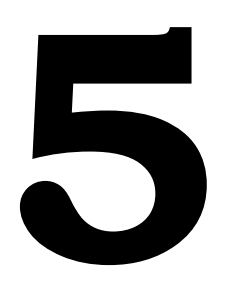

\section{Resting tremor classification and detection in Parkinson's disease patients}

This first work investigates the use of classification methods to identify tremors experienced by Parkinsonian patients to propose a new approach for demand driven stimulation. Feature extraction was conducted through a multilevel decomposition via a wavelet transform. Finally, artificial intelligence techniques were applied to feature selection, clustering of tremor types, and tremor detection.

The key contribution of this work is to present initial results which indicate, to a high degree of certainty, that there appear to be two distinct subgroups of patients within the group-1 of patients according to the Consensus Statement of the Movement Disorder Society on Tremor. Such results may well lead to different resultant treatments for the patients involved, depending on how their tremor has been classified.

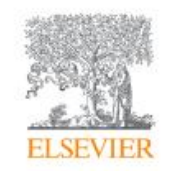

$$
\begin{gathered}
\text { Biomedical Signal Processing and Control } \\
\text { Volume 16, February 2015, Pages } 88-97
\end{gathered}
$$

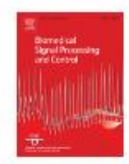

\section{Resting tremor classification and detection in Parkinson's disease patients}


everal previous works have studied diverse methods to detect and quantify PD $S$ tremors [105-107]. Most of them focus the analysis on external body signals such as accelerometry, electromyography (EMG) and/or electroencephalography (EEG) - not exploring what exactly is happening in the areas of interest inside the brain but conversely dealing with the question as a black-box problem. Fortunately, here I have access to sub-cortical data, which facilitates the applicability of the obtained results into real medical devices since we are directly dealing with brain signals.

\subsection{Tremor}

There are different kinds of tremor, depending on 1) the circumstances in which it appears: at rest, during maintenance of certain positions or while performing voluntary actions; 2 ) the affected body area: hands, arms and other body parts; and 3 ) the frequency at which the tremor manifests itself: low $(<4 \mathrm{~Hz})$, medium (4 $-7 \mathrm{~Hz})$ or high $(>7 \mathrm{~Hz})$ frequency bands. According to these three factors, tremor can be classified within a movement disorder pathology.

The Consensus Statement of the Movement Disorder Society on Tremor [108] categorises subtypes of tremor for this condition into three distinctly separate groups:

1. Resting tremor (RT), which is the most characteristic of PD tremors, occurs at a frequency band between $4 \mathrm{~Hz}$ and $6 \mathrm{~Hz}$ [109] and disappears when a voluntary movement is performed. Its presence is a good criterion for the diagnosis of $\mathrm{PD}$, since this sort of tremor is usually not associated with other pathologies. On the other hand, for the vast majority of PD patients, the resting tremor emerges along with postural and/or kinetic tremors at the same frequency. Therefore many studies simply assume that it is a continuation of the resting tremor under postural, kinetic conditions or vice versa [110-116].

Postural tremor takes place when the patient suffers a tremor episode maintaining a position against gravity, for instance keeping the arms $90^{\circ}$ horizontally relative to the trunk. Meanwhile, kinetic tremor occurs when the subject performs any voluntary movement.

2. The second group is made up of PD patients who have episodes of RT together with postural/kinetic tremor episodes at higher frequencies than the resting tremor, referred to as Essential Tremor (ET). Many research studies justify this since ET episodes can co-exist together with RT episodes in PD [117].

3. The last group includes patients who do not have resting tremor episodes. This subgroup of patients is only affected by kinetic and postural tremor episodes [118].

In this work, using clustering techniques, the existence of two patient subgroups within the group- 1 of patients mentioned above according to the Consensus Statement of the Movement Disorder Society on Tremor are shown. That is patients 
with resting tremor in the band 4-6 Hz. It is also shown that these group-1 patients can be clearly grouped into two further different subgroups. Unfortunately, I do not present any particular physiological reasoning behind this result/conclusion; merely it is an observation from the available signals. Nevertheless, we hope that this result can be used to conduct research on the existence of these sub-groups of patients and, above all, can be used to improve the treatment of PD.

Apart from this, it is also proposed, based on this classification, a tremor detection system that distinguishes between the aforementioned subgroups, obtaining better results (higher accuracy) than if clustering is not done (i.e., a detection system that does not segregate into subgroups) as shown in Section 5.3. This approach thus could potentially be used as an effective tool for categorising the DDS required.

\subsection{Data Preparation and feature extraction}

In this study, the non-transition subset of the dataset is used, as indicated in section 4.1.

Preprocessing

Before dealing with the data, some signal manipulations were needed. The preprocessing procedure is summarized in Figure 5.1 and explained below:

First the signals were down-sampled to the lowest sampling frequency used $(250 \mathrm{~Hz})$ since not all the files were originally sampled at 250 $\mathrm{Hz}$.

Secondly, a 3-30 Hz Chebyshev Type II passband filter was used on the LFP signals. LFP signals contain movement artefacts at $1-2 \mathrm{~Hz}$ and this set to 3 $\mathrm{Hz}$ the low cut-off frequency. Frequencies above the beta-band $(>30 \mathrm{~Hz})$ are considered to have little tremorrelated information [119]. By fixing the upper cut-off frequency at $30 \mathrm{~Hz}$ we excluded the $50 \mathrm{~Hz}$ line noise as well. We chose the Chevyshev type II filter because it does not produce any ripple in the passband and thus does not alter the frequency of the signal.

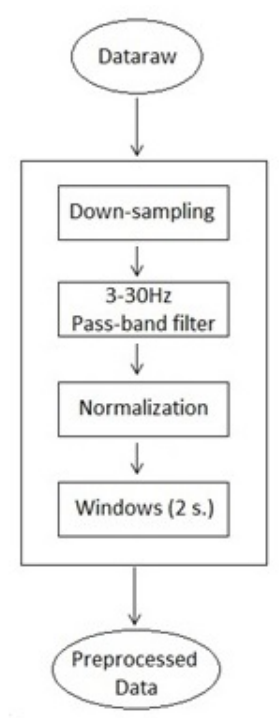

Figure 5.1: Preprocessing 
Third, an amplitude normalization is performed in order to reduce amplitude variations across patients, prior comparison between them.

Finally, I split the data into 2 seconds windows with $0 \%$ overlapping. We opted to use 2 seconds windows due to the number of samples available to us and the desired resolution. The window size is based on achieving a trade-off between the temporal resolution and the number of available samples. That is, the greater is the size of the window the higher temporal resolution we have at the expense of having fewer windows which are counter-productive for the machine learning algorithms. The use of 2 seconds windows provides an adequate temporal resolution since we can study what happens every $\frac{1}{2} \mathrm{~Hz}$, while we count with a significant number of windows - an average of 42 per patient. Since the sampling frequency is set to 250 $\mathrm{Hz}$, each window consists of 500 samples.

\section{Feature Extraction}

Once signals had been preprocessed, each window was characterized in terms of a set of features. To extract these features, signals could be analyzed in either the time or frequency domain. The Fast Fourier transform (FFT) is the most used tool for frequency domain analysis; however, temporal information is lost once the transformation is performed.

If the signal is non-stationary, such as LFP signals, both the temporal and frequency components contain relevant information about the signal. The Short-Time Fourier transform (STFT) divides the signal into windows and applies the FFT to each of them. Although we have a time-frequency representation of the signal, it has the restriction that the window size is fixed and resolution is thus limited by the selected window.

A Wavelet Transform (WT) is a multi-resolution transformation that uses a variable window size at each level. This allows us to get more information about the signal in the time-frequency (time-scale) domain. Motivated by this fact, WT was used in our experimentation.

In particular, the Discrete Wavelet Transform (DWT) was employed. The resolution was set to 6 levels, which is the maximum possible decomposition that can be performed considering a sampling frequency at $250 \mathrm{~Hz}$ (number of levels $\left.\leq \log _{2}(250 / 2)-1\right)$. Therefore each 2 seconds window is represented by six vectors $\left\{X_{i}\right\}_{(i=1)}^{6}$, which symbolize the wavelet coefficients at each of the levels. In fact, we dealt with the square value of the coefficients, which represents its power. For each of these vectors (levels), we calculated five features, which have proven to be valid in previous studies [120]:

- Energy: power sum of the coefficients at the $i-t h$ level. For a vector $X_{i}$ of 
length $n$, the energy is defined as:

$$
e_{i}=\sum_{k=1}^{n} X_{i}(k)
$$

- Average value: represents the mean value of the coefficients power at the $i-t h$ level. For a vector $X_{i}$ of length $n$, the average energy is defined as:

$$
\mu_{i}=\frac{1}{n} \sum_{k=1}^{n} X_{i}(k)
$$

- Variance: represents a dispersion measure from the mean energy at each level. For a vector $X_{i}$ of length $n$, the variance of the energy is defined as:

$$
\sigma_{i}=\frac{1}{n} \sum_{k=1}^{n}\left(X_{i}(k)-\mu_{i}\right)^{2}
$$

- First derivate: average value of the first derivate of the energy at each level. For a vector $X_{i}$ of length $n$, the average value of the first derivate is defined as:

$$
\delta_{i}=\frac{1}{n-1} \sum_{k=2}^{n}\left(X_{i}(k)-X_{i}(k-1)\right)
$$

- Entropy: represents the uncertainty value of the energy at each level. Let $\mathcal{X}=\{X, p\}$ a discrete space of probability. That is, $X=\left\{X_{i}, \ldots, X_{n}\right\}$ is a finite set in which each element has probability $p\left(X_{i}\right)$. Then, the Shannon entropy $s_{i}$ is defined as:

$$
s_{i}=-\sum_{k=1}^{n} p\left[X_{i}(k)\right] \cdot \log _{2} p\left[X_{i}(k)\right]
$$

Summarizing; each window of 2 seconds ( 500 samples) is characterized by 30 values ( 6 levels $\times 5$ features), which represents a $94 \%$ reduction of the input space. Mathematically, each sample can be represented by a vector, as shown below:

$$
\left[e_{1} \mu_{1} \sigma_{1} \delta_{1} s_{1}, \cdots, e_{6} \mu_{6} \sigma_{6} \delta_{6} s_{6}\right]
$$

\subsection{Proposed System \& Results}

\section{Clustering}

Clustering was only performed with tremor episodes. This can be justified based on two main reasons: 


\begin{tabular}{|l|c|c|c|c|c|c|c|}
\hline & \multicolumn{9}{|c|}{ Training Patients } & \multicolumn{2}{c|}{ Testing Patients } \\
\cline { 2 - 8 } & BE & MA & DC & RB & SW & GC & EP \\
\hline Cluster 1 & 1 & 2 & 20 & 0 & 1 & 0 & 0 \\
\hline Cluster 2 & 24 & 40 & 45 & 1 & 1 & 23 & 73 \\
\hline Cluster 3 & 1 & 0 & 0 & 1 & 38 & 2 & 4 \\
\hline Cluster 4 & 3 & 0 & 5 & 1 & 0 & 0 & 0 \\
\hline Cluster 5 & 0 & 7 & 18 & 79 & 12 & 3 & 10 \\
\hline Cluster 6 & 1 & 35 & 8 & 0 & 1 & 8 & 27 \\
\hline \hline Total Instances & 26 & 77 & 73 & 1 & 3 & 31 & 100 \\
Type A & $(96.3 \%)$ & $(91.7 \%)$ & $(80.2 \%)$ & $(1.2 \%)$ & $(5.7 \%)$ & $(86.1 \%)$ & $(87.7 \%)$ \\
\hline $\begin{array}{l}\text { Total Instances } \\
\text { Type B }\end{array}$ & 1 & 7 & 18 & 80 & 50 & 5 & 14 \\
& $(3.7 \%)$ & $(8.3 \%)$ & $(19.8 \%)$ & $(98.8 \%)$ & $(94.3 \%)$ & $(13.9 \%)$ & $(12.3 \%)$ \\
\hline
\end{tabular}

Table 5.1: Clustering Results: Training \& Testing Patients

1. It is understood that the samples from which we attempt to differentiate patients are tremor episodes. Non-tremor episodes were studied, and these are much more homogeneous and similar among patients. From this, we postulate that during non-tremor episodes, there is no significant difference in the subthalamus activity between healthy and Parkinsonian patients.

2. As a consequence of suppressing the observation period after the surgical procedure, only tremor episodes are currently available. This data can be gathered during the electrode implantation, as mentioned in Section 5.2. It prevents the possibility of training a neural network for a new patient. Only tremor samples would be available for this new patient, and the system would not be able to learn what non-tremor means in this case, impeding the automation of the tremor detection.

The goal at this point is to find out whether it is possible to cluster into groups the tremor instances for the set of patients. If so, this would indicate the existence of different types of patient, or different classes of resting tremor to be more precise.

The dataset employed was composed of seven patients; and 30, 84, 96, 82, 53, 36 and 114 tremor instances were available for each of them respectively.

\section{Clustering Results}

$\mathrm{K}$-means technique was selected since this is one of the most used clustering methods in practice. In short, this is an unsupervised system. The main goal of clustering algorithms is to sort the different instances into groups so that the degree of association between instances is maximized for the same group. That is, the goal is to group instances by proximity. This is performed by measuring distances between instances. In particular, the squared Euclidean distance is used as a metric.

In our experimentation, the clustering system was trained in order to group instances of five patients into six different clusters. The number of clusters was determined following the algorithm proposed by Jain and Dubes [121]:

1. Select an initial partition with K clusters; repeat steps 2 and 3 until cluster membership stabilizes. 
2. Generate a new partition by assigning each pattern to its closest cluster centre.

3. Compute new cluster centres.

The clustering results are conclusive since the groups obtained to facilitate the distinguishing of instances into two different types of tremor:

1. Tremor type A, which corresponds to clusters 1,2 and 6 .

2. Tremor type B, which corresponds to clusters 3 and 5 .

The tremor exhibited by each of the training patients (BE to SW) in our study belongs unequivocally to one and only one of these tremor types. Table 5.1 shows the results. Note that cluster 4 is not taken into consideration due to the tiny number of instances within it.

Once trained using data from 5 patients only, the system was tested with data from the two patients (GC and EP), which was not used during training. The results, displayed on the right side of Table 5.1, continue to show a clear tendency to cluster each of the patients into one of the groups previously found. In particular, in this case, both subjects belong to the group or type of tremor A.

From the results obtained in the clustering task, it can be concluded that each patient presents one particular type of tremor only. But it is interesting to point out that for all the patients, regardless of the fact that they are presumed to belong to a particular group, there are a very small number of tremor instances that are classified into the other existing group. This misclassification could be caused by physical or neurological causes, but the particular reason is unknown. Therefore, neuronal activity during tremor episodes can be, to some extent, different for the same patient while the physical symptom is the same. It may well be however that by further studying the exact nature of the tremor in each case this will reveal that there are also physical differences between types $\mathrm{A}$ and $\mathrm{B}$.

In Table 5.2, I summarize the average and standard deviation for the calculated multi-level features for each group of patients. By analyzing these results, we can conclude that the energy, average, variance, and first derivative are the features where the differences between patients are more significant. I have confirmed this by running an algorithm for feature selection. In detail, the Best-First and Correlation Feature Selection have been the algorithms used as attribute evaluator and search method, respectively.

Once executed, the selected features are: Energy (levels 1-4), Average (levels 3 and 4), Variance (level 3), First Derivative (levels 2-4). Therefore energy, average, variance and first derivative seem to be good distinguishers of subthalamic cell activity. Although, these ten values are the most representative, in our experimentation we finally used the whole set of features since the dimension of vectors (i.e., $1 \times 30)$ is manageable and slightly improved results are obtained. 


\begin{tabular}{|c|c|c|c|c|c|}
\hline \multirow{3}{*}{ Features } & \multirow{3}{*}{ Wavelets Levels } & \multicolumn{2}{|c|}{ Mean } & \multicolumn{2}{|c|}{ Standard Deviation } \\
\hline & & \multicolumn{2}{|c|}{ Tremor Type } & \multicolumn{2}{|c|}{ Tremor Type } \\
\hline & & A & B & $A$ & $\mathrm{~B}$ \\
\hline \multirow{6}{*}{ Energy } & Level 1 & 2.8794 & 3.5678 & 0.9643 & 1.9809 \\
\hline & Level 2 & 2.7769 & 1.5300 & 0.9284 & 1.0444 \\
\hline & Level 3 & 1.6990 & 0.4289 & 0.8238 & 0.4406 \\
\hline & Level 4 & 0.7595 & 0.1874 & 0.5471 & 0.1594 \\
\hline & Level 5 & 0.1270 & 0.0411 & 0.1201 & 0.0449 \\
\hline & Level 6 & 0.0023 & $7.4885 \times 10^{-4}$ & 0.0024 & $8.0716 \times 10^{-4}$ \\
\hline \multirow{6}{*}{ Average } & Level 1 & 5.8866 & 6.5612 & 1.0281 & 2.0794 \\
\hline & Level 2 & 5.7777 & 4.2117 & 0.9925 & 1.3368 \\
\hline & Level 3 & 5.0119 & 2.7299 & 1.0504 & 0.9913 \\
\hline & Level 4 & 4.0653 & 2.3625 & 1.1451 & 0.9953 \\
\hline & Level 5 & 2.4918 & 1.5018 & 1.0263 & 0.9079 \\
\hline & Level 6 & 0.4059 & 0.1612 & 0.3393 & 0.1524 \\
\hline \multirow{6}{*}{ Variance } & Level 1 & 5.9025 & 7.3970 & 2.1965 & 4.1806 \\
\hline & Level 2 & 5.8478 & 3.1928 & 1.8891 & 2.5782 \\
\hline & Level 3 & 3.6547 & 0.7607 & 1.9497 & 1.3641 \\
\hline & Level 4 & 1.4299 & 0.2845 & 1.2834 & 0.6132 \\
\hline & Level 5 & 0.1045 & 0.0360 & 0.1944 & 0.1922 \\
\hline & Level 6 & $3.8229 \times 10^{-5}$ & $1.3967 \times 10^{-5}$ & $8.5761 \times 10^{-5}$ & $7.7135 \times 10^{-5}$ \\
\hline \multirow{6}{*}{ First Derivative } & Level 1 & 8.9264 & 10.4965 & 2.0333 & 4.1639 \\
\hline & Level 2 & 9.2509 & 6.1841 & 1.9076 & 2.7438 \\
\hline & Level 3 & 7.6489 & 3.1558 & 2.0878 & 2.1637 \\
\hline & Level 4 & 5.1144 & 2.4593 & 2.1153 & 1.7638 \\
\hline & Level 5 & 1.6085 & 0.5211 & 1.4206 & 0.9605 \\
\hline & Level 6 & 0.0153 & 0.0048 & 0.0305 & 0.0250 \\
\hline \multirow{6}{*}{ Entropy } & Level 1 & 0.1120 & 0.1097 & 0.0112 & 0.0112 \\
\hline & Level 2 & 0.1076 & 0.1017 & 0.0117 & 0.0158 \\
\hline & Level 3 & 0.0758 & 0.0738 & 0.0065 & 0.0093 \\
\hline & Level 4 & 0.0493 & 0.0453 & 0.0037 & 0.0081 \\
\hline & Level 5 & 0.0299 & 0.0282 & 0.0025 & 0.0050 \\
\hline & Level 6 & 0.0181 & 0.0171 & 0.0013 & 0.0026 \\
\hline
\end{tabular}

Table 5.2: Statistical Analysis of Extracted Features 


\begin{tabular}{|l|c|c|c|c|c|c|c|}
\hline & \multicolumn{9}{|c|}{ Training Patients } & \multicolumn{2}{c|}{ Testing Patients } \\
\cline { 2 - 8 } & EP & MA & DC & RB & SW & GC & BE \\
\hline Cluster 1 & 38 & 9 & 0 & 0 & 3 & 1 & 22 \\
\hline Cluster 2 & 23 & 31 & 45 & 1 & 0 & 7 & 2 \\
\hline Cluster 3 & 3 & 1 & 0 & 1 & 37 & 2 & 1 \\
\hline Cluster 4 & 42 & 36 & 17 & 0 & 1 & 23 & 2 \\
\hline Cluster 5 & 8 & 7 & 18 & 79 & 12 & 3 & 0 \\
\hline Cluster 6 & 0 & 0 & 16 & 1 & 0 & 0 & 3 \\
\hline \hline Total Instances & 103 & 76 & 78 & 2 & 4 & 31 & 29 \\
Type A & $(90.4 \%)$ & $(90.5 \%)$ & $(81.3 \%)$ & $(2.4 \%)$ & $(7.5 \%)$ & $(86.1 \%)$ & $(96.7 \%)$ \\
\hline $\begin{array}{l}\text { Total Instances } \\
\text { Type B }\end{array}$ & 11 & 8 & 18 & 80 & 49 & 5 & 1 \\
\end{tabular}

Tremor type A: Clusters 1, 2, 4 and 6; Tremor Type B: Clusters 3 and 5

Table 5.3: Clustering Results: Extra Trial 1

\begin{tabular}{|l|c|c|c|c|c|c|c|}
\hline & \multicolumn{9}{|c|}{ Training Patients } & \multicolumn{2}{c|}{ Testing Patients } \\
\cline { 2 - 8 } & BE & EP & DC & RB & SW & GC & MA \\
\hline Cluster 1 & 1 & 3 & 0 & 1 & 38 & 2 & 1 \\
\hline Cluster 2 & 22 & 40 & 0 & 0 & 1 & 1 & 9 \\
\hline Cluster 3 & 2 & 17 & 43 & 1 & 0 & 7 & 22 \\
\hline Cluster 4 & 0 & 8 & 16 & 79 & 12 & 3 & 7 \\
\hline Cluster 5 & 4 & 0 & 12 & 1 & 2 & 1 & 0 \\
\hline Cluster 6 & 1 & 46 & 25 & 0 & 0 & 22 & 45 \\
\hline \hline Total Instances & 29 & 103 & 80 & 2 & 3 & 31 & 76 \\
Type A & $(96.7 \%)$ & $(90.4 \%)$ & $(83.3 \%)$ & $(2.4 \%)$ & $(5.7 \%)$ & $(86.1 \%)$ & $(90.5 \%)$ \\
\hline Total Instances & 1 & 11 & 16 & 80 & 50 & 5 & 8 \\
Type B & $(3.3 \%)$ & $(9.6 \%)$ & $(16.7 \%)$ & $(97.6 \%)$ & $(94.3 \%)$ & $(13.9 \%)$ & $(9.5 \%)$ \\
\hline
\end{tabular}

Tremor type A: Clusters 2, 3, 5 and 6; Tremor Type B: Clusters 1 and 4

Table 5.4: Clustering Results: Extra Trial 2

\section{Clustering verification}

In order to ensure that two different types of tremor exist in our patient dataset, I performed several clustering trials. In each experiment, I varied the training set of patients. The goal here was to ascertain if the two groups (type A and B) observed in the initial configuration remain irrespective of whether a different set of patients is chosen for the training phase.

In this section, some of the tested configurations are presented. Two different types are obtained in all the configurations. More precisely, in all of them, patients $\mathrm{RB}$ and $\mathrm{SW}$ are from a different type than patients $\mathrm{BE}, \mathrm{EP}, \mathrm{MA}, \mathrm{DC}$ and $\mathrm{GB}$.

The obtained results are summarized in Tables 5.3-5.6. The following considerations can be extracted when we analyze in depth the data:

- In Table 5.3, we can observe that patient DC has 16 instances in cluster number 6 . In this cluster, we do not find any instances of the remaining patients, so the determination of type A or B is, in principle, doubtful. We finally classify cluster 6 as type A, because the majority of instances of that patient DC are type $A$. Furthermore, the other two patients that have instances in that cluster (patients BE and RB), have more instances within type $A$. 


\begin{tabular}{|l|c|c|c|c|c|c|c|}
\hline & \multicolumn{4}{|c|}{ Training Patients } & \multicolumn{2}{c|}{ Testing Patients } \\
\cline { 2 - 8 } & BE & EP & DC & MA & SW & GC & RB \\
\hline Cluster 1 & 1 & 16 & 43 & 21 & 0 & 16 & 1 \\
\hline Cluster 2 & 22 & 41 & 9 & 14 & 0 & 2 & 6 \\
\hline Cluster 3 & 0 & 9 & 17 & 7 & 13 & 3 & 2 \\
\hline Cluster 4 & 3 & 4 & 1 & 1 & 38 & 2 & 12 \\
\hline Cluster 5 & 0 & 44 & 7 & 41 & 0 & 1 \\
\hline Cluster 6 & 4 & 0 & 19 & 0 & 2 & 1 & 1 \\
\hline \hline Total Instances & 27 & 101 & 78 & 76 & 2 & 31 & 8 \\
Type A & $(90.0 \%)$ & $(88.6 \%)$ & $(81.3 \%)$ & $(90.5 \%)$ & $(3.8 \%)$ & $(86.1 \%)$ & $(9.8 \%)$ \\
\hline Total Instances & 3 & 13 & 18 & 8 & 51 & 5 & 74 \\
Type B & $(10.0 \%)$ & $(11.4 \%)$ & $(18.7 \%)$ & $(9.5 \%)$ & $(96.2 \%)$ & $(13.9 \%)$ & $(90.2 \%)$ \\
\hline
\end{tabular}

Tremor type A: Clusters 1, 2, 5 and 6; Tremor Type B: Clusters 3 and 4

Table 5.5: Clustering Results: Extra Trial 3

\begin{tabular}{|l|c|c|c|c|c|c|c|}
\hline & \multicolumn{9}{|c|}{ Training Patients } & \multicolumn{2}{c|}{ Testing Patients } \\
\cline { 2 - 8 } & BE & MA & GC & RB & SW & EP & DC \\
\hline Cluster 1 & 1 & 36 & 0 & 0 & 1 & 24 & 15 \\
\hline Cluster 2 & 0 & 0 & 28 & 0 & 0 & 8 & 16 \\
\hline Cluster 3 & 3 & 0 & 0 & 1 & 0 & 0 & 2 \\
\hline Cluster 4 & 2 & 1 & 3 & 1 & 38 & 4 & 5 \\
\hline Cluster 5 & 24 & 40 & 1 & 1 & 1 & 68 & 38 \\
\hline Cluster 6 & 0 & 7 & 4 & 79 & 13 & 10 & 20 \\
\hline \hline Total Instances & 25 & 76 & 29 & 1 & 2 & 100 & 69 \\
Type A & $(92.6 \%)$ & $(90.5 \%)$ & $(80.5 \%)$ & $(1.2 \%)$ & $(3.8 \%)$ & $(87.7 \%)$ & $(73.4 \%)$ \\
\hline Total Instances & 2 & 8 & 7 & 80 & 51 & 14 & 25 \\
Type B & $(7.4 \%)$ & $(9.5 \%)$ & $(19.5 \%)$ & $(98.8 \%)$ & $(96.2 \%)$ & $(12.3 \%)$ & $(26.6 \%)$ \\
\hline
\end{tabular}

Tremor type A: Clusters 1, 2, and 5; Tremor Type B: Clusters 4 and 6

Table 5.6: Clustering Results: Extra Trial 4 
- In Table 5.6 and only observing the training results, it is questionable whether patient GC belongs to type A or type B since the instances of this patient are mostly in cluster 2 , which does not have instances from other patients.

From this, we might conclude that type $C$ also exists. Nevertheless, if we observe the test results, we can clearly determine that cluster 2 belongs to type A - although this decision cannot be determined during the training phase. On the other hand, "this uncertainty" does not appear in the other tables evaluated, where all patients clearly show membership, in training and also in testing, to one of the existing groups (type A or B) - even this patient.

Note that in this configuration we have not considered cluster 3 since the number of samples belonging to this cluster is negligible.

- In all the configurations, we can observe that all the patients have instances in both types. But adding the total number of instances, it can be seen that every patient clearly belongs to one type. This has been the criterion about membership of a patient to type A or type B.

- We have mentioned that although the patients clearly belong to one type, they have instances from the other type. If we pay more attention to this, it is remarkable that this fact is more noticeable for the patients of type $A$ than for type B. That is, patients of type A have a membership, although clear, which is less strong than patients of type $B$.

Summarizing, and after conducting in-depth experimentation, we conclude that only two types of tremor exist in our patients' dataset.

\section{Detection (Proposed System)}

Previous works have studied the possibility of demand-driven stimulation in DBS, as opposed to continuous stimulation which is the present norm. In this article, due to the knowledge we have acquired about tremor types, we find it logical to integrate these results with the tremor detection task. Therefore, the proposed system combines the tasks of clustering and detection. For this, I have designed a detection algorithm in which two different neural networks are trained (using tremor and non-tremor instances): one per each patient type, A and B, as shown in Table 5.1. The number of instances that are evaluated on each network is shown in Table 5.7 in Section 5.3, which arguably gives the clearest indication of the existence of different tremor types. In the validation phase, each patient (tremor instances) is evaluated by the clustering module and depending on this result, each sample is assessed in the corresponding network. Although mostly each patient belongs to one type, all of them have tremor instances in both groups, as shown in Table 5.8 in 


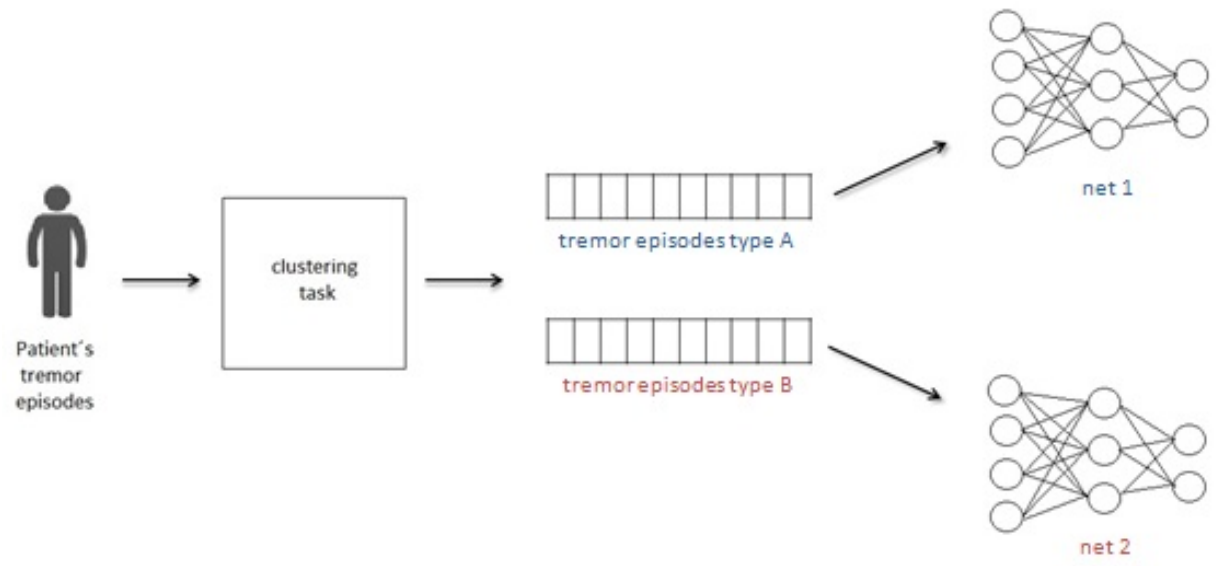

Figure 5.2: System Operation Scheme

Section 5.3. In Table 5.9 we computed the degree of accuracy in tremor detection for both networks. The weighted average value is used since each patient did not have the same number of instances in each network.

The overall system operation scheme (tremor classification type and tremor detection), applied for each patient, is shown in Figure 5.2.

\section{Neuronal Network Design}

A neural network was chosen given its history of successful application in pattern recognition problems. In our case a Back Propagation Multi-Layer Perceptron with one hidden layer with 16 neurons was chosen. The mathematical statement of the MLP is determined by the following equation, from which the corresponding outputs to the inputs provided to the network are calculated:

$$
\gamma=\varphi\left(\sum_{i=1}^{n} \omega_{i} x_{i}+b\right)=\varphi\left(\omega^{T} x+b\right)
$$

where $x$ is the input vector, $\omega$ is the vector of weights, $b$ is the bias parameter and $\varphi$ is the network's activation function. Here I choose $\varphi$ one of the two most frequently functions used, the sigmoid function $\frac{1}{\left(1+e^{-x}\right)}$.

MLP networks are normally used to solve supervised learning problems. That is when the set of inputs and corresponding outputs are completely known - in our case, using 30 features per window as input, and the presence or absence of tremor as output - the system learnt the relationship between inputs and outputs.

Therefore the feature extraction procedure plays a key role. It is only as a result of this that the network can be adequately trained to identify the tremor and non- 


\begin{tabular}{|l|c|c|}
\hline & Network 1 & Network 2 \\
\hline Used Instances & 520 & 278 \\
\hline \% Total Accuracy & 87 & 81.5 \\
\hline \% Tremor Accuracy & 97 & 82.5 \\
\hline \% Non-Tremor Accuracy & 94 & 82 \\
\hline
\end{tabular}

Table 5.7: Training of Networks 1 and 2 (with cross-validation)

\begin{tabular}{|c|c|c|}
\hline \multirow[t]{2}{*}{ Patient ID } & \multicolumn{2}{|c|}{ Number of Instances } \\
\hline & $\begin{array}{c}\text { Network } 1 \\
\text { Type A }\end{array}$ & $\begin{array}{c}\text { Network } 2 \\
\text { Type B }\end{array}$ \\
\hline Patient BE & 26 & 1 \\
\hline Patient MA & 77 & 7 \\
\hline Patient GC & 31 & 5 \\
\hline Patient RB & 1 & 80 \\
\hline Patient SW & 3 & 50 \\
\hline Patient EP & 100 & 14 \\
\hline Patient DC & 73 & 18 \\
\hline
\end{tabular}

Table 5.8: Number of Instances in Network 1 and 2

tremor windows. If the features are poor, the system will fail in its attempt.

As shown in Figure 5.2, I used two neuronal networks. The input to both of the networks, in the training phase, consisted of the whole signal (tremor and nontremor episodes) for each of the five training patients. More precisely, Network 1 is trained with data from patients of type $A$ : patients $B E, M A$ and $G C$, with a total of 520 instances for all of them. For its part, network two is trained with patients of type B: patients RB and SW, with a total of 278 instances.

For the training parameters of both networks, I opted for training with $80 \%$, and testing with the remaining $20 \%$ of samples. These percentages were chosen because the goal at this phase was only to train the networks with the validation process at a later stage. Note that we did not use $100 \%$ of the samples for training in order to avoid over-learning.

The obtained results are summarized in Table 5.7 and Table 5.8.

\section{Network Validation}

Once the two networks were trained, we validated them. At this stage, only tremor episodes were used. This has a twofold justification. On the one hand, tremor detection is the main goal of our system. On the other hand, and due to the abolition of the semi-implanted period in much present-day neurostimulator implantation, if the system is employed with a new patient, only tremor episodes would be available.

The whole set of patients was tested in the two networks, and validation was carried out using cross-validation with ten folds. Table 5.9 shows the accuracy obtained per patient, dividing their tremor episodes between the networks, which is based on the clustering results. The degree of overall success is the weighted average. All patients exceeded $73 \%$ accuracy, achieving $100 \%$ accuracy in the group of patients 


\begin{tabular}{|l|c|c|c|}
\hline \multirow{2}{*}{ Patient ID } & \multicolumn{3}{|c|}{ Accuracy } \\
\cline { 2 - 4 } & $\begin{array}{c}\text { Network 1 } \\
\text { Type A }\end{array}$ & $\begin{array}{c}\text { Network 2 } \\
\text { Type B }\end{array}$ & $\begin{array}{c}\text { \% Patient } \\
\text { weighted average }\end{array}$ \\
\hline Patient BE & 100 & 100 & 100 \\
\hline Patient MA & 100 & 85.7 & 98.8 \\
\hline Patient GC & 93.6 & 80 & 91.7 \\
\hline Patient RB & 100 & 76.6 & 76.9 \\
\hline Patient SW & 100 & 90 & 90.6 \\
\hline Patient EP & 99 & 64.3 & 94.7 \\
\hline Patient DC & 70 & 88.9 & 73.8 \\
\hline \hline Overall Performance & 92.0 & 81.4 & 89.5 \\
\hline
\end{tabular}

Table 5.9: Testing of Networks 1 and 2 - Proposed System

used in training (20\% of their instances were not employed in training), and $94.7 \%$ in the case of the patients not utilized during the training. From the obtained results, it seems that the system works very well with high overall accuracy $(89.5 \%$ of overall performance).

\subsection{Discussion (Performance Evaluation)}

In order to validate our proposal, we compared the obtained results with those obtained when patient data was classified with a neural network without tremor distinction. Therefore, no classification was made distinguishing between types of tremor and the clustering of the patients was omitted.

In this case, the same type of neuronal network was trained using the 30 features as previously described: a Multi-Layer Perceptron with $80 \%$ of samples for training and $20 \%$ for testing, and with one hidden layer composed of 16 neurons. The network was trained with data from the first five patients ( $B E$ to $S W$ ), which have a tremor and non-tremor episodes. In this group of patients, there were two different types of tremor episodes, as was shown before, but this fact was omitted in order to perform a comparison with our proposed system. That is, we trained only a network with all members of this group of 5 patients, instead of training two different networks (each per type of patient/tremor).

After training, the network was tested with the tremor episodes of each of the seven patients.

Comparing the results obtained using this approach (see Table 5.10) with those obtained in the previous section (see Table 5.9), it can be concluded that the performance improves when clustering is applied prior to detection. Generally, there is an increment in the average accuracy of detection, and greater stability is achieved for all the patients.

\subsection{Conclusions}

In this work, the study has focused on Resting tremor through the LPF signals collected from the subthalamus in patients diagnosed with tremor dominant idiopathic 


\begin{tabular}{|l|c|}
\hline Patient ID & Accuracy \\
\hline Patient BE & 100 \\
\hline Patient MA & 100 \\
\hline Patient GC & 83.3 \\
\hline Patient RB & 73.17 \\
\hline Patient SW & 92.45 \\
\hline Patient EP & 92.98 \\
\hline Patient DC & 55.20 \\
\hline \hline Overall Performance & 83.2 \\
\hline
\end{tabular}

Table 5.10: Detection without Tremor Type Clustering

PD. All the patients present the same symptomatology - RT in the frequency band between 4 and $6 \mathrm{~Hz}$. We aimed to look for the existence of sub-group(s) of patients (tremor sub-group). From our experimentation and as a result we showed the existence of two subgroups of patients within the group-1 of patients according to the Consensus Statement of the Movement Disorder Society on Tremor [108].

It is acknowledged here that the total number of patients involved in this study is relatively small. That said, there were no cases which disproved the hypothesis and the results obtained are, we feel, strongly supportive. However, the next step is to extend the study considerably in order to see if all PD cases fall into one or other of the two subgroups as categorised or if there appear to be any exceptions.

The physiological causes of this can be a matter for further study, and we would not wish to speculate on them here. In particular, it would be interesting to study, at the neurological level, what are the particular causes for this distinction concerning the underlying subthalamic activity. Nevertheless, I hope that this result may be used as the basis to advance the research into types of patients and tremors in Parkinson's disease. Moreover, this research advance may help to develop improved and more specialized therapies to treat PD.

Finally, using the obtained results, we propose a novel tremor detection mechanism that distinguishes between subgroups of patients and obtains a higher performance (accuracy) than a detection system that treats all the patients in the same single group. Therefore, the proposed system seems an effective tool to assist in demanddriven deep brain stimulation. 



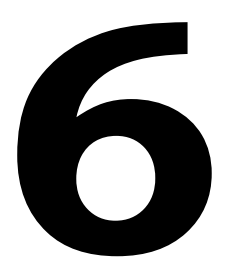

\section{A fuzzy inference system for closed-loop deep brain stimulation in Parkinson's disease}

The goal of the designed system in this chapter is to be able to detect when the patient is suffering a tremor episode. The synchronization level between the subthalamic and the muscular activity have been employed in the detection of tremor onset. The results of evaluating the indexes on each windows represent the inputs to the designed system. Finally, a fuzzy inference system is applied for tremor detection. Results are favourable, reaching accuracies higher than $98.7 \%$ in the $70 \%$ of the patients.

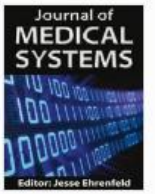

Journal of Medical Systems

November 2015, 39:155 | Cite as
A Fuzzy Inference System for Closed-Loop Deep Brain Stimulation in Parkinson's Disease




\subsection{Methods}

In this study the non-trasition subset of the dataset are used, as indicated in the section 4.1 .

\section{Data Preprocessing}

Before dealing with the data, it was necessary to carry out a series of preprocessing techniques to eliminate noise, filter the bands and highlight events of interest. In this section the techniques used and the reasons for their need is presented.

Normalization. The files of the dataset vary in amplitude between patients. This is due to the idiosyncrasies of the signal and the symptomatology of the disease. It is, therefore, necessary to perform normalization of the data, such that the posterior processing and obtained results are comparable among different patients. Therefore we normalized all channels separately so that all of them maintain their oscillatory properties, but the mean and the variance were normalized to 0 and 1 , respectively.

Resampling. The sampling rate varies between $250-1000 \mathrm{~Hz}$ throughout our patient dataset. A resampling is realized in order to make the posterior processing the same for all the data and so that the results are comparable. The files were resampled to $250 \mathrm{~Hz}$, which was the minimum sampling frequency present in all the files.

Filtering. Due to the different characteristics and nature of the LFP and EMG signals, the filtering pipeline was also different.

For LFP band-pass filtering between 2 and $45 \mathrm{~Hz}$ with a 500 order FIR filter designed using a hamming window were performed. LFP signals contain movement artefacts around $1 \mathrm{~Hz}$, so we set the low cut-off frequency at $2 \mathrm{~Hz}$. On the other hand, by fixing the upper cut-off frequency at $45 \mathrm{~Hz}$ we excluded the line noise (in Europe $50 \mathrm{~Hz}$ ) as well.

The EMG signal was filtered in two steps. In the first stage, a band-pass order 500 FIR filter between 30 and $125 \mathrm{~Hz}$ were applied. This frequency band was selected due to the nature of the EMG signal, consisting in burst starting at $30 \mathrm{~Hz}$. The upper edge of the filter is the maximum allowed frequency of the signal, due to the Nyquist theorem. This signal was then rectified to obtain a baseband signal describing the muscular activity.

Rectification is nonlinear processing that modifies the signal spectrum. When dealing with EMG this method intensifies the signal power at low frequencies [122]. Hilbert transform of the filtered EMG signal was calculated and constructed the analytic signal, $A(x)$. From this analytic signal, we calculated the envelope, which represents the instantaneous oscillatory energy of the EMG signal. This envelope is often used as an indicator of muscular activity.[123]. 
Finally, this envelope was filtered with the same filter applied to LFP in order to be able to compare both signals.

Windowing. Tremor episodes experienced by patients in our dataset are not isolated in files of one nature. Therefore in our dataset, some files contain both tremor and rest episodes due to the patient experiencing both states during the recording period.

The data obtained was split into 2 seconds of contiguous windows without overlapping. The window size is based on achieving a trade-off between temporal resolution and the number of available samples. The use of 2 seconds windows provides an adequate temporal resolution (with a frequency step of $1 / 2 \mathrm{~Hz}$ ), while counting on a significant number of windows - an average of 42 per patient. The signal sampling frequency was set to $250 \mathrm{~Hz}$, so each 2 seconds window contained 500 samples.

\section{Synchronization measures as features}

To understand more how the brain works, it is useful to observe it working naturally. We can do that by means of several techniques based on imaging (such as functional magnetic resonance imaging or positron emitting tomography) or based on the electrical or magnetic signals generated in the brain (such as Electroencephalography or Magnetoencephalography). In the case of this study, I count with two simultaneous signals: local field potentials and electromyography. LFP measures the aggregate presynaptic and postsynaptic activity of a population of neurons; meanwhile, EMG measures the electrical activity realised due to skeletal muscle contraction.

The EMG signal is more reliable regarding tremor detection as opposed than raw LFP signal since the first one measures directly the muscular activity, which is higher under tremor episodes. Moreover, some indexes reflect an increase of synchronisation levels under tremor episodes, in comparison with rest states. We consider here two synchronisation measures as inputs to a fuzzy controller, mutual information and bicoherence.

The selection of the indexes was based on performance. I tested several synchronisation indexes, including correlation, coherence [124] and phase-locking value [125]. The selected ones were Mutual Information and Bicoherence since they are the ones better distinguish between the tremor and resting states. Their mathematical description can be seen in 2.3.2.

\subsection{Fuzzy System}

Traditionally the field of predicate logic works by dividing the output space into disjoint sets. Thus, any predicate $P$ applied to a collection $U$, is associated with a precise set denoted as $P \subset U$. This can be described through the membership 
function $\mathrm{P}$ whose value is determined by the belonging to $\mathrm{P}$ of the different elements $u \in U$ which is defined by:

$$
\mu_{p}(u)= \begin{cases}1 & \text { if } u \in P \\ 0 & \text { otherwise }\end{cases}
$$

So if $\mu_{p}(u)$ is 1 , the predicate is true. Whereas if it is zero, the predicate will be false.

Designing systems employing classical logic has the problem that they are too rigid. This rigidity is a limitation in the sense that it is challenging to model systems based on precise rules when the domain is not determined by precise inputs and/or outputs. Therefore classical logic turns out to be insufficient to solve some problems. From this need, the definition of fuzzy sets and fuzzy logic has appeared. It builds models that are particularly useful to work with uncertainty in a more natural way.

A fuzzy predicate, evaluated on a collection $U$, is associated to a fuzzy set $V \subset U$ , which is defined by the function $\mu_{v}(u)$ This function determines the membership function of the elements of $\mathrm{V}$ to $\mathrm{U}$, as a matter of degree.

$$
u_{v}: U \rightarrow[0,1]
$$

Thus, a fuzzy set is one in which the elements do not belong completely to only one set, but do belong to that sets to a certain extent. As a result, to work with fuzzy sets, we define the degrees of membership of the different elements to each set.

A fuzzy control system was chosen for the detection of resting tremor motivated by two main reasons which are:

- The impreciseness can be a source of uncertainty. EMG and LFP time series, despite having been meticulously collected by the medical team, may have certain levels of impreciseness because of the noise. This may be due, for example, to differences in skin impedance. As a result, this could alter the values of electromyography. Fuzzy methods are able to manage the imprecision present in the data.

- It allows for variability in the data. As a preliminary study, we have explored how the different synchronisation index works on each patient. Although generally, the trend remains the same for all patients, there is not a threshold that determines from which value of the index we can consider that the patient is experiencing a tremor episode. So there is a short-range of values on which the output cannot be easily determined, and these can be either a tremor or rest episode. We know that if the system output is in the range zero to 0.2 , the input was a resting episode. Similarly, if the output is greater than 0.4, the input was almost surely a tremor episode. However, what happens when the output is within the approximate interval $[0.2-0.4]$ ? 
Taking into consideration both situations in the design of the detection system, for the problem addressed in this work it can be beneficial when we attempt to minimise the number of false negatives. A false negative would mean that our system has detected an input as a resting episode, when in fact, it is a tremor one. In these situations, the resultant controller would not provide stimulation to the patient when it is actually needed. Because of that, we are aiming to diminish the number of false negatives. Indeed this is the most critical situation.

False positives, on the other hand, are not so much of a problem. In such instances, an input would be detected as a tremor episode when in fact it was actually a normal resting period. In this case, the controller would provide unnecessary stimulation. The negative of this is that useful power is being used up by the device for no reason. In the limit, with lots of such cases, the controller would tend to the present case of continual stimulation. But although this is not a desirable situation, it is not critical as the current system works in this mode with the patient not suffering any backside.

\subsection{System}

The specific components of a fuzzy controller are shown in figure 6.1. MI (mutual information) and $\mathrm{BICOH}$ (bicoherence) are the inputs to the system. These inputs are the results of evaluating the synchronization measures to each window of the preprocessed raw data obtained, as described in the previous section.

The central block of the figure represents the fuzzy system itself. It is composed of three modules on the design of a Mamdani fuzzy inference system: Fuzzification, Rule inference and Defuzzification [126].

- Fuzzification of the input variables. This step evaluates the conditions of the rules and turns the inputs to membership degrees for each set.

- Rule Inference. This step comprises rule evaluation and the aggregation of the generated outputs.

- Defuzzification. Finally, it is necessary to carry out defuzzification. This process transforms the aggregate fuzzy output set to a single number. Doing this, we translate the result obtained to one that has a sense in our domain.

Based on the obtained output, the system evaluates if the input corresponds or not to a tremor episode. When the onset of a tremor episode is detected, the neurostimulator will give the order to stimulate. In fact, it is also possible to predict such onset and hence apply stimulation to stop the tremor actually occurring in the first place [127].

\section{Fuzzification}

This step evaluates the conditions of the rules and turns the inputs to membership degrees for each set. The membership function defines the fuzzy sets. Its determination is a matter of design and tends to be subjective. It must be designed with 


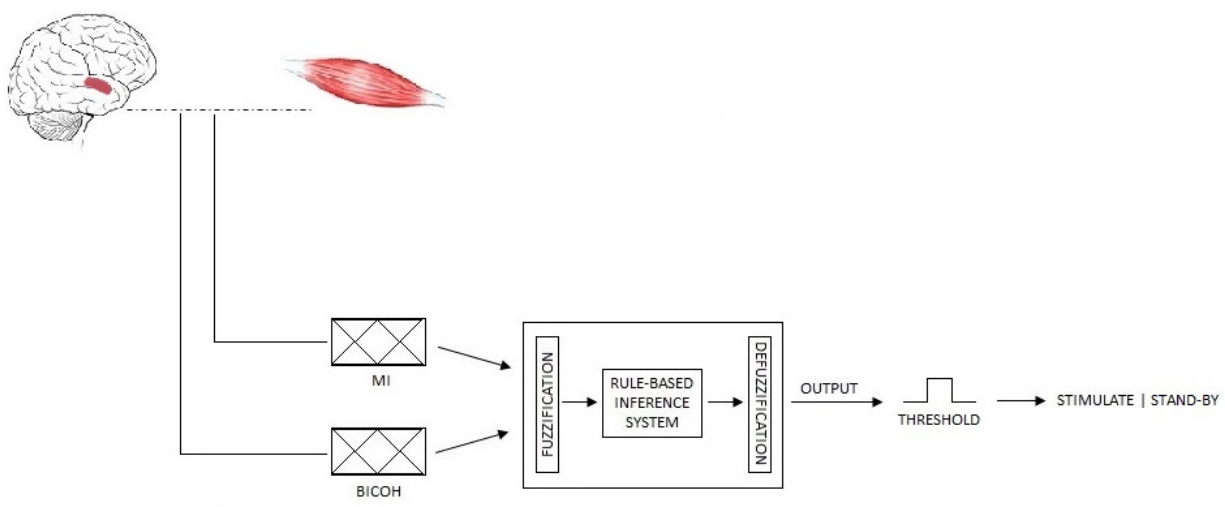

Figure 6.1: System operation scheme

full knowledge of the domain and the nature of the inputs which are provided to the fuzzy control system. Therefore certain aspects have been taken into account: [128]

- The sets are sufficiently wide to allow noise in the measurement.

- A certain amount of overlap is desirable; otherwise the controller may run into poorly defined states, where it does not return a well-defined output.

The selection of the membership function for each set is based on knowledge of the dataset. In that sense, we have chosen spline-based functions for the NO-TREMOR input set, given by:

$$
f(\text { input })= \begin{cases}1 & \text { if input }<=1 \\ 1-2\left(\frac{\text { input }-0.1}{0.1-1}\right)^{2} & \text { if } 0.1<=\text { input }<=\frac{0.1+0.2}{2} \\ 1-2\left(\frac{\text { input }-0.2}{0.2-0.1}\right)^{2} & \text { if } \frac{0.1+0.2}{2}<=\text { input }<=0.2 \\ 0 & \text { if } \text { input }>=0.2\end{cases}
$$

In the same way we define spline-based curves for the TREMOR input, given by:

$$
f(\text { input })= \begin{cases}0 & \text { if } \text { input }<=0.05 \\ 2\left(\frac{\text { input }-0.05}{0.2-0.05}\right)^{2} & \text { if } 0.05<=\text { input }<=\frac{0.05+0.2}{2} \\ 1-2\left(\frac{\text { input }-0.2}{0.2-0.1}\right)^{2} & \text { if } \frac{0.1+0.2}{2}<=\text { input }<=0.2 \\ 0 & \text { if } \text { input }>=0.2\end{cases}
$$

The output represents the state of the neurostimulator. Depending on the tremor state of the patient, the stimulator can be either stimulating or kept waiting, just listening (We denominate this state as stand-by henceforth). To model this, we opted for trapezoidal-shaped membership functions, defined by: 


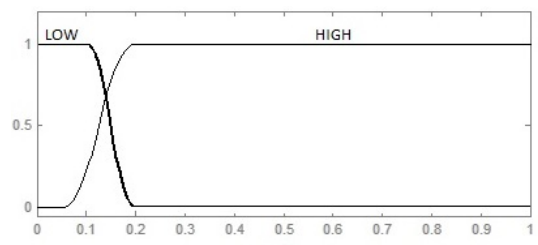

Figure 6.2: Membership for Mutual Information Input

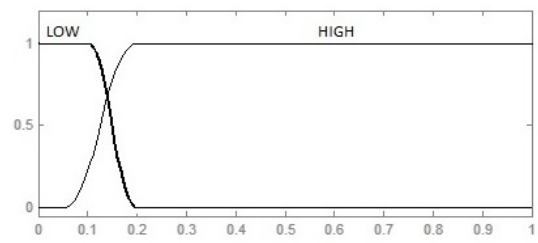

Figure 6.3: Membership for Bicoherence Input

$$
\begin{aligned}
& f(\text { output })= \begin{cases}0 & \text { if output }<=0 \\
1 & \text { if } 0<=\text { output }<=0.1 \\
\frac{0.2-\text { output }}{0.2-0.1} & \text { if } 0.1<=\text { output }<=0.2 \\
0 & \text { if } 0.2<=\text { output }\end{cases} \\
& f(\text { output })= \begin{cases}\frac{0 u t p u t-0.05}{0.2-0.05} & \text { if output }<=0.05 \\
1 & \text { if } 0.05<=\text { output }<=0.2 \\
\frac{1-\text { output }}{1.01-1} & \text { if } 1<=\text { output }<=1.01 \\
0 & \text { if } 1<=\text { output }\end{cases}
\end{aligned}
$$

Rule Evaluation

This step comprises rule evaluation and aggregation of the generated outputs. Evaluation of the rules uses fuzzy operators, which are applied over the inputs to calculate the trigger force of the rules. The result is a fuzzified value for each of the rules.

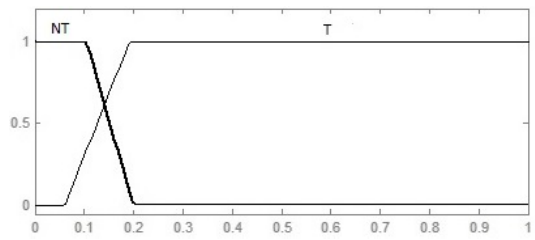

Figure 6.4: System's Output Membership 


\begin{tabular}{|c|c|c|c|}
\hline Rule Number & MI & BICOH & DECISION \\
\hline $\mathbf{1}$ & LOW & LOW & STAND-BY \\
\hline $\mathbf{2}$ & HIGH & LOW & STIMULATE \\
\hline $\mathbf{3}$ & LOW & HIGH & STIMULATE \\
\hline $\mathbf{4}$ & HIGH & HIGH & STIMULATE \\
\hline
\end{tabular}

Table 6.1: System's Rules

Fuzzy Operators . Fuzzy logic is a superset of classical logic. Classical logic uses absolute operators to calculate the output of a given input, that is, only Boolean values for the output are contemplated. Meanwhile fuzzy logic employs fuzzy operators which contain the values 0 and 1 as extremes, but also takes into account all the intermediate values, thereby allowing for uncertainty. I use the AND operator, which in our case works with the minimum method.

Definition of the rules. The rule set defines how the inputs are related to the outputs. Fuzzy rules differ from standard rules in that the antecedent of a classical rule must be $100 \%$ true in order that the consequent can be evaluated. However, in fuzzy rules, the antecedent can be partially true, which does not in itself enable or prevent the trigger of the rule, simply that the consequent is partially true.

During the design stage, the definition process of the fuzzy rules usually requires an adjustment process to optimize the performance of the system. The defined rules are shown in Table 6.1, where each row represents a rule. As described in the previous section, the synchronization indexes can take the fuzzy value of low or high. Meanwhile, the overall/final output is either Stimulate or Standby.

Evaluation and Aggregation . Once rules have been evaluated we have one physical output for each rule. But having different output sets does not provide any directly meaningful information, thus it is necessary to perform an aggregation of the outputs. This step performs a unification combining the membership functions of all the rule consequents. In our case we have chosen the maximum method to perform the aggregation.

\section{Defuzzification and Thresholding}

Finally, it is necessary to carry out a defuzzification. This process transforms the aggregated fuzzy output set to a single number. Doing this, we turn the result obtained into one that has a sense in our domain. In this step, we have opted for the centroid method, which calculates the centre of the area of the obtained fuzzy set.

The output of the system represents the degree of synchronization between LFP and EMG. This value does not; however, itself determine the level of stimulation from the device. Therefore, a thresholding module is added. This module compares the output to a preset threshold, in this way: 


$$
\text { device }- \text { decision }= \begin{cases}\text { stimulate } & \text { if output }>=\text { threshold } \\ \text { stand }- \text { by } & \text { otherwhise }\end{cases}
$$

As will be seen in the next section, this threshold can be the same for all of the patients involved, or it can be adjusted individually. This last option can be of interest as it can improve tremor detection. It has been previously shown that there appear to be different types of Parkinson's Disease [129]. It may well be, therefore, that the threshold is linked to the disease classification.

\subsection{Results}

In this section we present the obtained results. By considering the range of the outputs, we can define four ranges: $[0-0.2),[0.2-0.3),[0.3-0.4)$ and $[0.4-1]$. We evaluate the number of false positives, false negatives, tremor accuracy, no-tremor accuracy and global accuracy for each range.

A false positive occurs when the system determines that the input was a tremor instance, whilst in reality, it was a no-tremor instance. Analogously, false negatives are the cases in which the system considers the input corresponds with a no-tremor episode, but in fact, the patient was trembling.

As previously mentioned, the final determination as to whether the system provides stimulation or not depends on whether the output is greater than a fixed threshold or not. The operation of the indexes, although very similar, is not exactly the same across the patients. Therefore, the threshold determination is critical for the accuracy of the system.

With this in mind, I studied here how the system works by determining the threshold in two different ways: 1) globally, fixing a global threshold for all the patients (so we take all the patient dataset into account together) and 2) Individually for each patient, taking the instances of each patient separately.

\section{Global Results}

By defining the system taking into account all the patients together, through experimentation, we found that the best value for the threshold was 0.4 . This value minimised the number of false positives below the threshold and the number of false negatives above it. This can be observed in Figure 6.5, where the output of the fuzzy system for each input is depicted. The numerical results are presented in Table 6.2.

The accuracy of tremor detection (named as tremor accuracy in the table) can be improved by lowering the threshold, getting then lower no-tremor accuracy. But, as have been mentioned before, for the patient's comfort, it is preferable to ensure that any time there is a tremor episode the neurostimulator is working. 


\section{Results per patient}

In this section, the analysis is performed by running the system for each patient individually. As for the global results, we show the numeric values in Table 6.3. This time, we include the column threshold, which indicates from which output value the system decides to apply stimulation. The graphical results are illustrated in Figure 6.6.

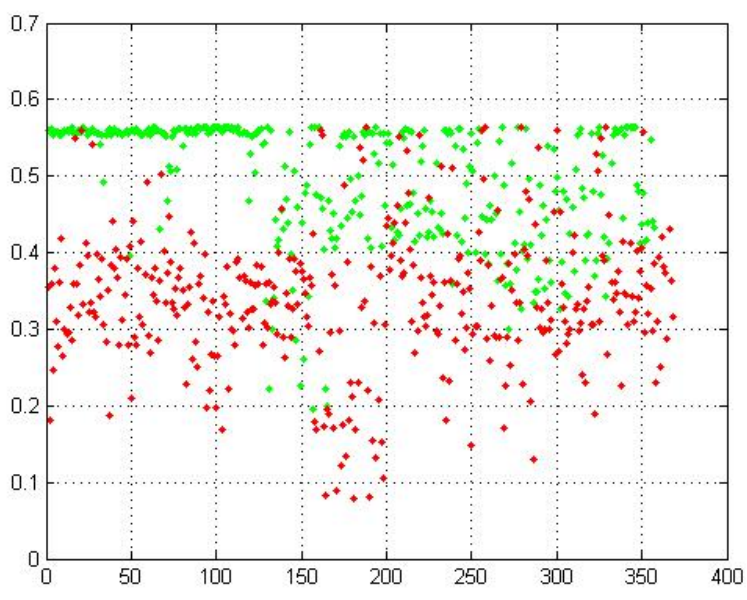

Figure 6.5: Output of the system for all the segments of all the patients. The vertical axis indicates the normalised output of the fuzzy system. Red dots represent no-tremor segments. Green dots represent tremor segments.

\begin{tabular}{|c|c|c|c|c|c|c|c|}
\hline Interval & $\begin{array}{l}\text { Number } \\
\text { of NT In- } \\
\text { stances } \\
\text { (red dots) }\end{array}$ & $\begin{array}{l}\text { Number of } \\
\text { T Instances } \\
\text { (green } \\
\text { dots) }\end{array}$ & FP & FN & $\begin{array}{l}\text { GLOBAL ACCU- } \\
\text { RACY }\end{array}$ & $\begin{array}{l}\text { TREMOR ACCU- } \\
\text { RACY }\end{array}$ & $\begin{array}{l}\text { NO-TREMOR } \\
\text { ACCURACY }\end{array}$ \\
\hline$[0-0.2)$ & 29 & 1 & \multirow{3}{*}{$42(12.2 \%)$} & \multirow{3}{*}{ - } & \multirow{3}{*}{$87.8 \%$} & \multirow{5}{*}{$88.2 \%$} & \multirow{5}{*}{$82 \%$} \\
\hline$[0.2-0.3)$ & 78 & 6 & & & & & \\
\hline$[0.3-0.4)$ & 195 & 35 & & & & & \\
\hline$[0.4-1]$ & 66 & 314 & - & $66(17.4 \%)$ & $82.6 \%$ & & \\
\hline$[0-1]$ & & Global & ccuracy & & $85.1 \%$ & & \\
\hline
\end{tabular}

Table 6.2: Global Results 


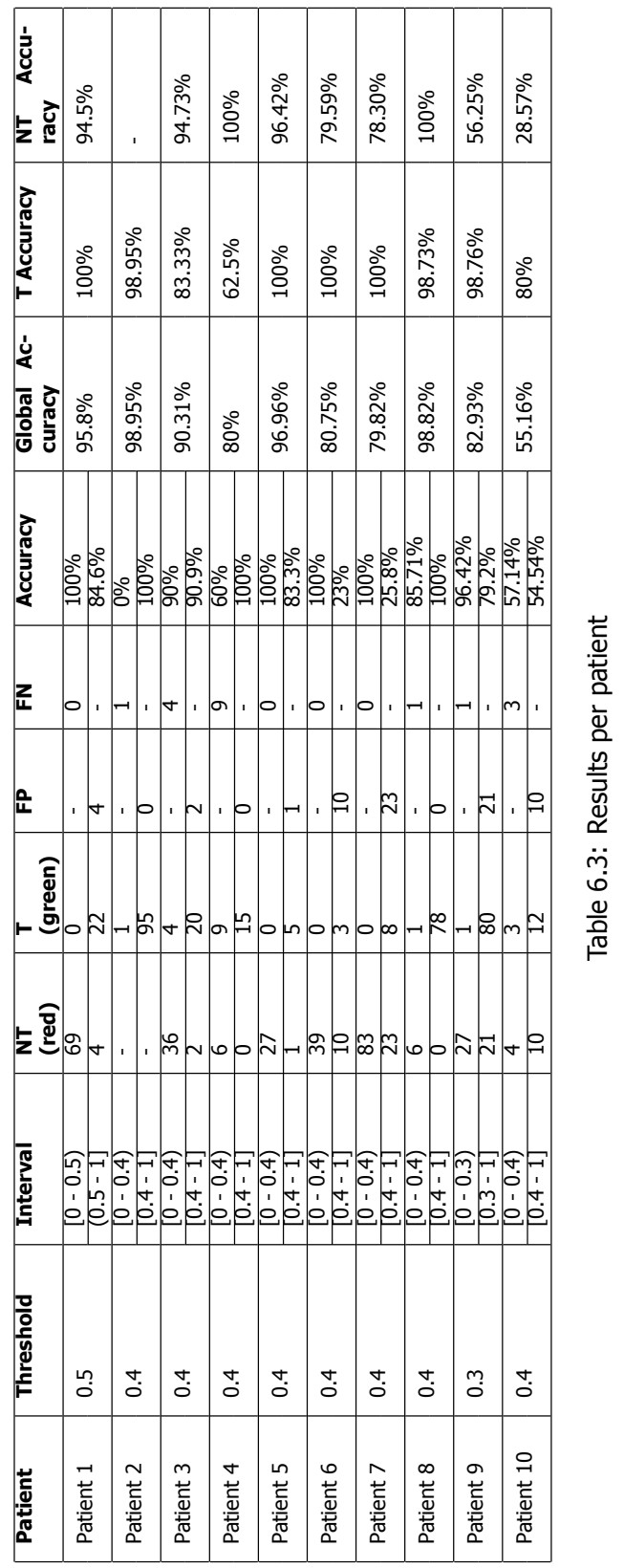


6. A fuzzy inference system for closed-loop deep brain stimulation in

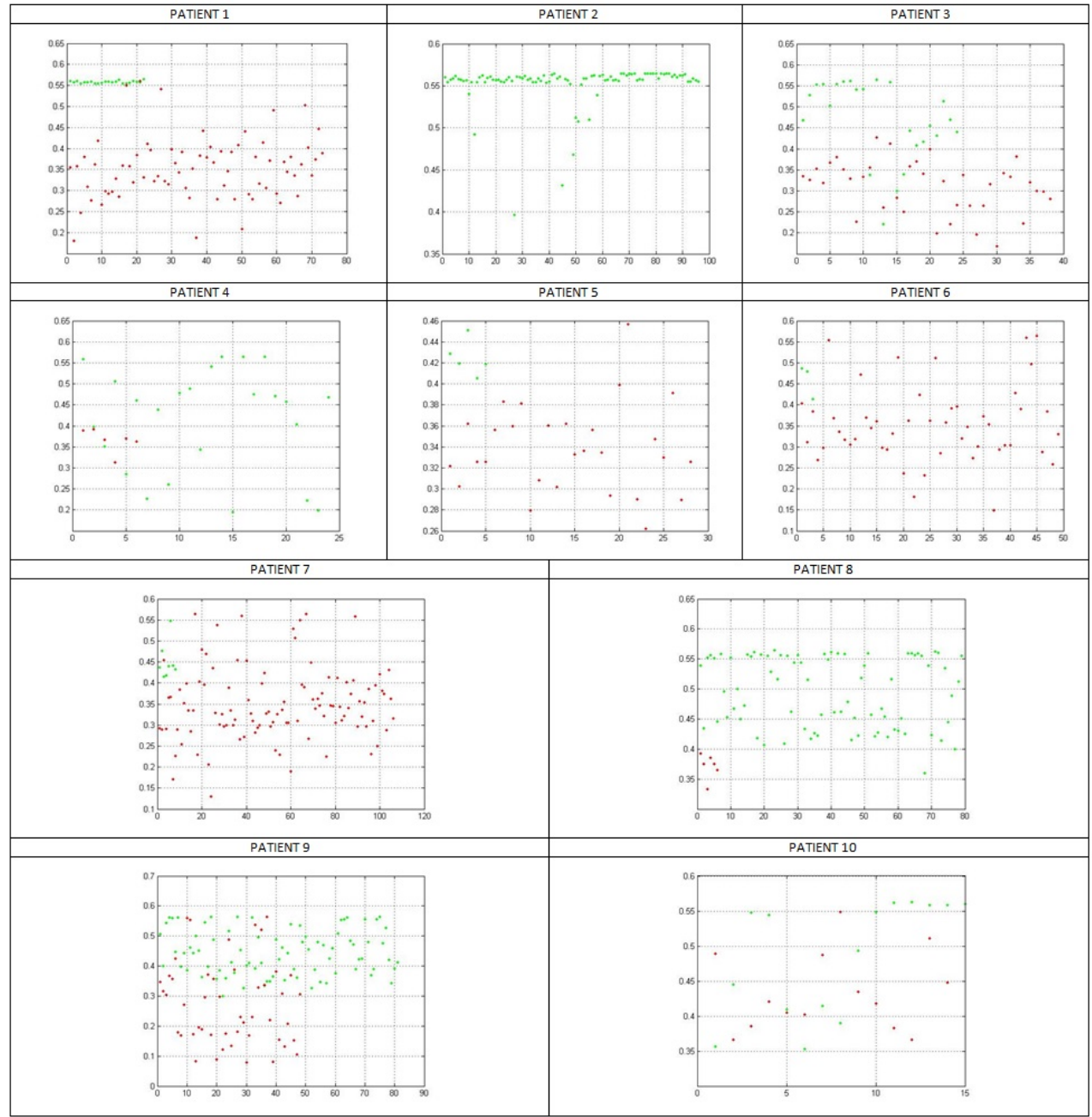

Figure 6.6: Output of the system evaluated per patient. Vertical axis indicates the normalised output of the fuzzy system. Red dots represent no-tremor segments. Green dots represent tremor segments.

\subsection{Discussion}

The system is able to reach an accuracy of $100 \%$ in tremor detection for 4 out of 10 of the patients. For the other 3 out of 10 of the patients, the number is almost $99 \%$. For 2 out of 10 of the patients, the accuracy reaches $80 \%$. Unfortunately in the case of one patient, the system was only able to detect $62 \%$ of the tremor episodes automatically.

Moreover, the system is also able to reach high levels of accuracy for no-tremor 
episodes, as shown in the last column of Tables 6.2 and 6.3. This implies an improvement of $82 \%$ (for the global evaluation) in comparison with the neurostimulators used nowadays. This improvement is even higher when we evaluate the system for each patient, reaching $100 \%$ in some cases.

The accuracy differences among patients are due to the fact, as can also be observed in the figures, that there is a clear separation between episodes of tremor and rest in some patients, while this segregation is not so clear in others. The physiological cause of this is unknown at present and could be due to a misplacing in the electrode positioning. However, showing such differences points to areas of further study.

With patient number 4 , the accuracy of tremor detection of only $62.5 \%$ was achieved; however, for this patient, the accuracy of $100 \%$ was achieved for no-tremor detection. Therefore a solution to improve the performance in these situations could be to lower the threshold. This would though slightly increase the false positives but increase the tremor detection accuracy, which is the priority. As we previously pointed out, an increase in the false-positive detection rate would not be harmful to the patient. So, despite this, the performance of the system would be superior to the one offered by the solutions currently used.

\subsection{Conclusions}

In this work a real-time resting tremor detection system has been proposed, which could be the first step towards demand-driven stimulation, providing more intelligent healthcare for patients who suffer from Parkinson's Disease.

The results are favourable, in the sense that $100 \%$ accuracy in tremor detection can be achieved in almost half of the cases. In fact, only 3 out of the 10 cases we obtained an accuracy lower than $98.7 \%$. Moreover, even in those cases, the system allowed a gain adjustment which increased the accuracy. For all the patients, a system such as the one proposed in this paper would improve the stimulation performance compared to the current stimulation systems, which basically consist of continuous stimulation.

In this study, I used synchronization measures as features for tremor detection. The two measures which are most effective for tremor detection were selected. It would be desirable to carry out an in-depth study on the level of synchronization between basal ganglia and muscle effectors in order to unveil why in some patients, the coupling between signals is not so clear. This knowledge would help in the development of demand-driven stimulation device, enabling it to be more stable across the patient spectrum. 



\section{7}

\section{Non-linear dynamical analysis of resting tremor for demand-driven deep brain stimulation}

Local field potentials (LFPs) have been widely studied to investigate deviations from the typical patterns of healthy brain activity. However, the inherent dynamics of the Sub Thalamic Nucleus (STN) LFPs and their spatiotemporal dynamics have not been well characterized. In this work, I study the non-linear dynamical behaviour of STN-LFPs of parkinsonian patients using $\varepsilon$-recurrence networks. RNs are a non-linear analysis tool that encodes the geometric information of the underlying system which can be characterised (for example using graph theoretical measures) to extract information on the geometric properties of the attractor.

\section{sensors}

\section{Open Access Article}

\section{Non-Linear Dynamical Analysis of Resting Tremor for Demand-Driven Deep Brain Stimulation}

\footnotetext{
Carmen Camara $1,2,3, \star, \dagger \square$, Narayan P. Subramaniyam $3, \dagger \square$, Kevin Warwick $4 \bowtie \odot$, Lauri Parkkonen ${ }^{3} \square$, Tipu Aziz ${ }^{5} \square$ and Ernesto Pereda $2,6 \square$
} 
ocal field potential (LFPs) have been widely studied. While some researchers L have explored the spectral power of the neural oscillations, usually assuming the existence of pathology when its range deviates from those present in control subjects $[130,131]$, others have studied the connectivity between the neurons within the STN [94, 132, 133]. However, the inherent dynamics and spatiotemporal profile of STN-LFPs have not been characterised for its application to a demand-driven DBS system. In the cases of dynamical diseases, algorithms from the non-linear dynamical analysis are particularly attractive to find out biomarkers that differ between normal and pathological states of the disease [134].

In this work, time series analysis methods derived from non-linear dynamics are applied to the study of the STN activity under the different tremor states. A moving window recurrence network (RN) analysis is carried out to capture such rich dynamical behaviour. RNs are constructed based on the recurrences in the phase space. By applying network analysis methods, the dynamically relevant structures of the time series data can be studied, extracting the geometrical properties of the attractor [135]. The study of the topological structure of the RN allows us to infer the complexity of the dynamics associated with the STN-LFP time series.

Methods based on RN constitute an excellent approach to analyse the structural complexity of neural signals, as they can be applied to short and non-stationary data, since the network properties, such as global clustering coefficient, transitivity, or assortativity can still be reliably estimated. This makes RNs an ideal method for capturing the dynamical transitions in neural data. Previous works have demonstrated the applicability of this method in detecting the onset of epileptic seizures $[136,137]$.

The present study aims to answer two main questions: 1) Do the dynamics of STNLFPs have a permanent character or do they change depending on the movement state that the patient is in?. 2) Would it be possible to characterise some feature in the neural signal, which can aid in predicting the onset of the tremor?

\subsection{Data Preparation}

\section{Dataset}

In this study the trasition subset of the dataset are used, as indicated in the section 4.1. It consists of four files that collect neural information while the patient transits from NT to TO. They allow us to characterise the dynamical transitions by identifying the different states. Especially interesting is the fact of being able to detect the TO state, and distinguish it from T, as it is the first step in preventing or suppressing the tremor by means of closed-loop DBS, before the tremor actually starts up for the patient. 


\section{Signal Preprocessing}

The sampling rate of the recordings varies between $250-1000 \mathrm{~Hz}$. In order to make the results comparable, we started resampling the recordings so that after this, we could apply the same processing procedure to all the data. To avoid redundant managing information, and according to the Nyquist theorem, downsampling to $125 \mathrm{~Hz}$ was performed. After that, STN-LFP recordings were filtered with a 500 order [2-45] $\mathrm{Hz}$ band-pass FIR filter designed with Hamming window in which two seconds of real data as padding are used. This step eliminates the movement's artefacts (around $1 \mathrm{~Hz}$ ) and the line noise $(50 \mathrm{~Hz}$ in Europe). Finally, the filtered data was segmented into windows of two second, using overlapping of $90 \%$. Using this level of overlapping, we can seize with a high temporal resolution the dynamics of the signal.

The EMG signal during tremor is composed of bursts having frequency peaks at $30 \mathrm{~Hz}$ and above. The data were filtered using a two-pass procedure with a highpass filter above $30 \mathrm{~Hz}$ of order 500 and designed using a Hamming window. Then the signal was rectified using the Hilbert envelope. Finally, the rectified signal was filtered with an FIR 2-45 Hz filter, getting the EMG signal at low frequencies.

\subsection{Recurrence Networks}

A dynamical system is a model in which the current state depends on the previous states and the transitional laws between them followed by the system. The state is defined through the values taken by the system variables. By connecting the different states through which the system passes, the trajectory of the system is obtained, which can be represented in the well-known phase space of the system [54].

The phase space trajectory of a dynamical system can be reconstructed from a scalar time series, by taking $m$ time-lagged observations of it. This is the wellknown Taken's embedding theorem [138]:

$$
x(n)=(u(n), u(n+\tau), \ldots, u(n+(m-1) \tau))
$$

where, $x(n) \in \mathbb{R}, \tau$ is the embedding delay and $m$ is the embedding dimension. Taken's theorem does not specify the values that $\tau$ and $m$ should take. Here, we make use of two widely used methods to set these parameters.

\section{Embedding delay}

The selection of $\tau$ has to be performed carefully, to avoid redundancy in the consecutive variables of the delayed vector. When $\tau$ is too small, no new information is extracted between successive observations, while if $\tau$ is too high, continuous observations are disconnected. To find an optimal value of $\tau$ we used auto-mutual information. Given a scalar time series, $x_{n}$ with $n$ samples, one can define the auto mutual information function as follows [139]: 

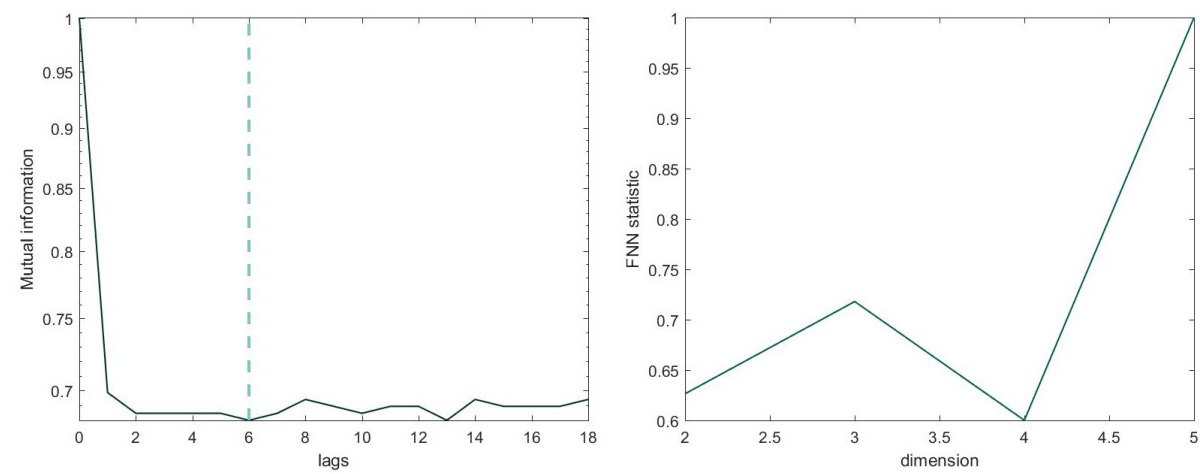

Figure 7.1: Optimal value of the parameters for an exemplary window. Left: optimal delay $\tau$ calculated with auto-mutual information. The dashed line determines the first local minimum $(\tau=6)$. Right: the minimum embedding $m$ employing the FNN method. At $m=4$, the FNN statistic is 0 .

$$
M I(\tau)=\sum_{n=0}^{N-1} p\left(x_{n}, x_{n+\tau}\right) \log \frac{p\left(x_{n}, x_{n+\tau}\right)}{p\left(x_{n}\right) p\left(x_{n+\tau}\right)}
$$

where $p(\cdot)$ stands for probability. The optimal value for the embedding delay is the value of $\tau$ at which $M I(\tau)$ reaches the first local minimum [140,141].

\section{Embedding dimension}

For the calculation of the optimal embedding dimension, we used the false nearest neighbourhood (FNN) method [142], which consists of calculating the number of points along the trajectory that are neighbours for different values of $m$. The value of $m$ at which the percentage of false neighbours becomes zero (or arbitrarily small, due to the effect of noise) is considered the optimal value of $m$. In order to construct RNs of the same size, the same values of $\tau$ and $m$ are set across windows of each file, allowing us to compare them. Figure 7.1 shows the auto-mutual information and the minimum embedding $m$ using the FNN method for an exemplary channel.

\section{Recurrence network}

From the state space, it is possible to build a complex network, on which graphtheoretical measures can be computed. In this study, a $\varepsilon$-RN is reconstructed. These kind of networks are a subtype of proximity networks, in which the vertices are represented by state vectors and the edges between the vertices are defined based on the mutual closeness between different state vectors in the phase space $[135,143]$. We can find different proximity networks, depending on how the concept of mutual closeness is defined. In the case of a $\varepsilon$-RN, a fixed phase space distance $\varepsilon$-centred around a vertex $i$ (a state vector in phase space) is defined [144]. All the vertices that fall within this volume are connected to the vertex $i$ by an edge. Such a network is both undirected and symmetric.

$\varepsilon$-RNs are based on the recurrences in phase space and are obtained by reinterpret- 
ing the recurrence matrix as the adjacency matrix of a complex network [135, 144]. A recurrence matrix represents the distance between the pairs of state vectors. It can be defined as [145]:

$$
R_{i, j}=\Theta\left(\varepsilon-\left\|x_{i}-x_{j}\right\|\right)
$$

where $\varepsilon$ is the recurrence threshold, $\Theta(\cdot)$ is the Heaviside function and $\|\cdot\|$ is a distance norm. Here we used the maximum norm as a distance norm. Instead of fixing $\varepsilon$, we fix the recurrence rate $R R=0.03$, so that we obtain RNs with a similar number of edges across windows, which makes it possible to compare them. This threshold determines the maximum spatial distance of neighbouring states.

The recurrence matrix is binary and symmetric. Each vertex $i$ represents a state vector $x_{i}$. Entry is 1 if the distance between two states is less than the defined threshold, 0 otherwise.

The adjacency matrix can be obtained from the recurrence matrix by removing the self-loops, that is, subtracting the identity matrix:

$$
A=R-I
$$

$A$ represents an undirected, unweighted complex network known as a recurrence network. From it, we can characterize the dynamically invariant properties of the neural dynamical system by using graph-theoretical methods.

\subsection{Network Measures}

By applying network analysis methods, the dynamically relevant structures underlying the time series can be studied by extracting the geometrical properties of the attractor. The network measures used in this study are presented in this section.

\section{Global Clustering Coefficient}

The local clustering coefficient for a vertex $i$ represents the probability that two randomly chosen vertices $j$ and $q$ are themselves, neighbours. The local clustering coefficient of a vertex $i$ can be given by:

$$
C_{i}=\frac{\sum_{j, q} A(i, j) A(j, q) A(q, i)}{k_{i}\left(k_{i}-1\right)}
$$

where $k$ is the degree of a vertex. Interpreting this coefficient in the context of RNs, $C_{i}$ is a measure of the fraction of vertices in the $\varepsilon$ - neighbourhood of a given vertex that is themselves mutually $\varepsilon$-close (within the same circumference). $C_{i}$ is averaged over all the vertices in the network to obtain the global clustering coefficient:

$$
C=\frac{1}{N} \sum_{I=1}^{N} C_{i}
$$




\section{Transitivity Dimension}

The concept of transitivity is very similar to that of clustering. It measures the fraction of triples in the network that form triangles. The main difference is that the transitivity coefficient is normalised by the value of the whole network, a quality that makes transitivity more robust compared to clustering against the presence of nodes with low degree. The transitivity is defined by [146] as:

$$
T(G)=\frac{3 \delta(G)}{T(G)}
$$

where $T(G)$ and $\delta(G)$ are the total number of triples and triangles in the network respectively. In terms of the recurrence matrix, $T$ can be defined as:

$$
T=\frac{\sum_{i, j, q=1}^{N} A(i, j) A(j, q) A(q, i)}{\sum_{i, j, q=1}^{N} A(i, j) A(q, i)}
$$

\section{Assortativity}

A network is assortative if the vertices with a similar degree tend to connect. The fact that a recurrence matrix is assortative means that the density of states within the $\varepsilon$-neighbourhood changes slowly and continuously. This coefficient is calculated by the Pearson product-moment correlation of the vertex degrees on either end of all the edges [147]:

$$
A=\frac{\frac{1}{N} \sum_{j>i} k_{i} k_{j} A(i, j)-\left[\frac{1}{N} \sum_{j>i} \frac{1}{2}\left(k_{i}+k_{j}\right) A(i, j)\right]^{2}}{\frac{1}{N} \sum_{j>i} \frac{1}{2}\left(k_{i}^{2}+k_{j}^{2}\right) A(i, j)-\left[\frac{1}{N} \sum_{j>i} \frac{1}{2}\left(k_{i}+k_{j}\right) A(i, j)\right]^{2}}
$$

Moving window $\varepsilon$-recurrence network analysis

In this work, a moving window $\varepsilon$-recurrence network analysis was used to compute the global measures $C, T, A$. The time series was previously divided into 2 seconds window with $90 \%$ overlap. In order to get the temporal profile of global network measures, we assigned the global measure to the mid-point of each window. $\tau$ and $m$ has been set on the first local minimum of the auto-mutual information and FNN method respectively, and the recurrence rate $R R$ was set to 0.03 .

For determine whether it is possible to anticipate the beginning of a tremor episode, a moving median filter over the signals $C, T, A$ was applied. This filtering allows to smooth out short-term fluctuations and to highlight the real transients in the STNLFP signal. The \pm 2 and \pm 3 standard deviation of the moving median filter was established to determine the statistical significance of the transients (peaks henceforth). Figure 7.2 shows the results of the analysis of one of the recordings.

Clustering $(C)$ : We witnessed a low level of $C$ during $N T$, that increased abruptly a few seconds before the onset of the TO $(p<0.01)$. The peaks describe an abrupt increase in the non-linearity in the system (for simplicity we will talk about peaks 

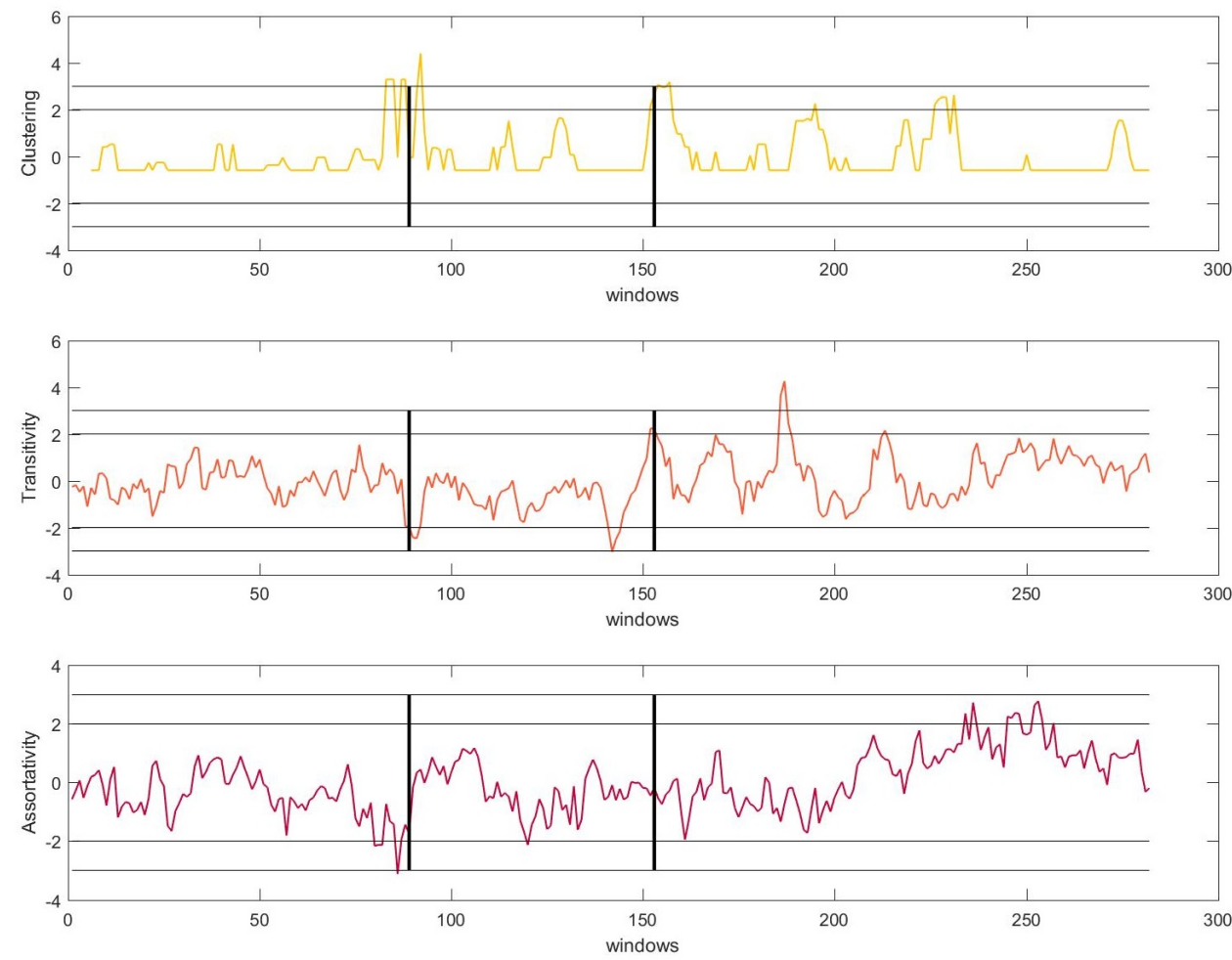

Figure 7.2: Moving window $\varepsilon$-recurrence network analysis showing the median moving average of clustering, transitivity and assortativity, before, during and after the start of the tremor, in this order.

The left and right black vertical line represents the transition fromNT to TO and from TO to $T$, respectively. The horizontal lines represent the \pm 2 and \pm 3 standard deviation thresholds for statistical significance 
instead of an increase in the non-linearity henceforth). Thereupon the level of $C$ decreased again, although it remained higher than its level during NT. A few seconds before the onset of the resting tremor $T$, we again detected an increase of $C$ $(p<0.01)$. During the $T$ section significant peaks $(p<0.05)$ were detected.

Transitivity $(T)$ : The levels of transitivity remain insignificant during NT. In both transition periods, we detected an increase in the level of $T(p<0.05)$. During the tremor section, the amplitude of the peaks increased, surpassing in some cases the +3 standard deviation.

Assortativity $(A): A$ has the same behaviour as transitivity, in that its fluctuations remained insignificant during the entire $N T$ period. However it marked the transition windows by becoming significant $(p<0.05)$ and increasing in amplitude in TO and $T$.

Equivalent results were found for all files. Importantly we found significant peaks before the transition of movement conditions for all the measures and files. These peaks are significant at $99 \%$ and $95 \%$ levels. Which imply that in all cases, the onset of the tremor can be predicted in advance by this method. Based on the obtained results, we can draw the following conclusions:

- The trends of the different measures are quite similar since all of them exhibit a shift in their dynamics near the beginning of the TO episode and before the $T$ episode. All measures detect the tremor efficiently before its appearance and therefore, before the patient shows any physical symptoms. This fact makes these measures good candidates for their application in a demand-driven DBS system.

- During tremor episodes, $T$ and $A$ display a growing trend, while $C$ exhibits the behaviour of shifting its dynamics more abruptly. The behaviour during NT and $T O$ are similar across all the measures.

\subsection{System}

The objective of the system is to detect the tremor through STN-LFP signal recorded by the electrodes, through the network features studied. The aim is to provide stimulation as soon as symptoms are detected, or ideally shortly before, and to stop stimulation as soon as atremororous instances are sensed. In this way, the system will be efficient concerning the treatment of symptomatology as well as with the use of the battery.

As previously mentioned, it is within the section labelled as $T$ when the patient begins to experience physically appreciable tremor, as determined by clinicians with expertise in movement disorders.

From the moment a tremor window arrives until the system classifies it as such and orders the stimulation to begin, a few seconds may pass. Therefore, it would be interesting to detect within the STN signal some event that anticipates the tremor episode. i.e. the ideal solution would be to detect the tremor before the patient 
a)

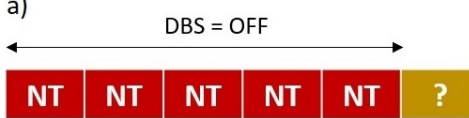

b)

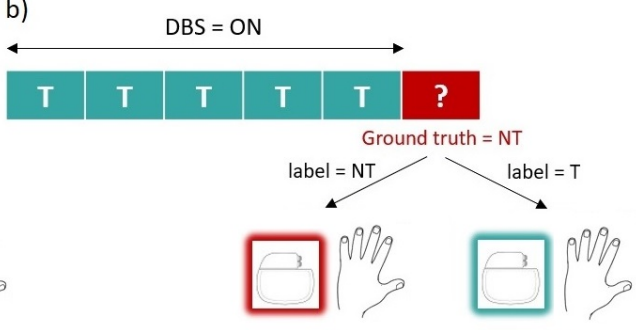

b1

Figure 7.3: This figure represents the four cases that can take place in our system when turning on/off the stimulation. a) The IMD is not stimulating and a TO sample arrives (the ground-truth of the sample is therefore TO). If the system fails to classify that sample, the stimulation will remain OFF, and the patient will begin to tremble after a few seconds (scenario a1 in the figure). If on the contrary, the system correctly identifies the sample as TO, it will order to start the stimulation (scenario a2 in the figure). b) If while the system is stimulating, an NT sample arrives: (the ground-truth of the sample is therefore NT): If the system correctly detects this new clinical state, it will turn OFF the stimulation, as it is no longer necessary (scenario b1 in the figure). While if the detection fails, the system will continue to stimulate (scenario b1 in the figure). However, in this case, contrary to what happens in scenario a1, this will have no physical effects on the patient.

begins to physically experience it, using those seconds to turn on the stimulation. In other words, ideally, a demand-driven DBS system must detect the tremor when the patient is in TO episode.

With this goal in mind, the dynamics of the STN signal have been studied following the methods presented in section 5 , since as we have seen, the network measures have proved to be outstanding capturing changes in the non-linearity of the system. These changes are especially noteworthy in the TO state, detecting a peak that reaches values of the significance of $p<0.01$ in all the studied recordings.

\section{Start and Stop Stimulation Decision}

To be valid for its purpose, the system must be able to recognise two main conditions: NT and TO instances. Notice that we have three classes (NT, TO and T); however, the system only needs to learn the first two to be able to carry out the two instructions that the system performs: start and stop stimulation. Notice also that the accurate detection of $T O$ is more critical than the detection of NT. This is because if a delay exists in stopping the stimulation as a consequence of not correctly classifying an instance of $N T$, it will not have any effect on the symptomatology, going unnoticed by the patient. However, the same delay in detecting a tremor instance would lead to the patient experiencing tremor as many seconds as windows the system needs to perform a correct classification. The system has to avoid this situation, therefore, and give the accuracy shown detecting a peak of non-linearity within the TO state, the system will base the decision to start stimulation on the presence of that peak. These possible scenarios are depicted in figure 7.3. 
NT state, making a system based solely on this method, not capable of detecting the stop condition. This would lead to a situation in which the IMD would stimulate in a continuous way (which is the current state we are trying to improve). Therefore, to detect the stop condition, the system will make use of an SVM classifier trained per patient using ten folds cross-validation. Support vector machines (SVM) is an algorithm that creates a non-linear discriminative classifier, determined by an optimal hyperplane that separate the instances of different classes, implicitly mapping the inputs into high-dimensional feature spaces (the well-known kernel trick) [148]. Once the system has learnt the mapping function, the new and unlabelled instances will be mapped into some of the created regions, adopting the label of that region.

\section{System Model}

The system operates as follows: Assuming the system is running, at time $t$ a new signal window arrives at the IMD. It preprocesses the signal as described in section 2 , calculates the network measures as described in the sections 4 and 5 and store the results in memory - Notice that the system only maintains in memory the four windows before which it is evaluated-. If DBS is OFF, the system has to decide if it turns on the stimulation or continues just sensing signal. For that, it averages the current window with the four previous ones. Depending on the results: a) If the result exceeds two standard deviations from the subject's baseline, a non-linearity peak has been detected, indicating that the patient is within TO state, and therefore the decision taken by the system will be to turn on the stimulation. b) The system will continue in standby otherwise.

Nevertheless, if in time $t$ the DBS in ON, the decision that the system must make is whether to turn off the stimulation. For this purpose, the trained SVM model will classify the sample. If it belongs to NT, the IMD will order to stop the stimulation. It will continue stimulating otherwise. The flow diagram of the system operating mode is depicted in figure 7.4.

\section{System Performance}

This section discusses the validity of the proposed system. Validity reflects the accuracy of the system, and it is measured by sensitivity and specificity. Sensitivity is the proportion of true positives and specificity is the proportion of true negatives that are correctly identified by the system. Besides, we are interested in measuring the false positive (FPR) and false negative (FNR) rates. FPR ( $\alpha$ or type I error) measures the percentage of cases in which the null hypothesis is correct but is rejected. While FNR ( $\beta$ or type II error) measures the percentage of cases in which the null hypothesis is false but is accepted.

In a demand-driven DBS system, the two main actions to be taken are when to turn on and when to turn off the stimulation. The validity of the model in each of these actions is evaluated here. 


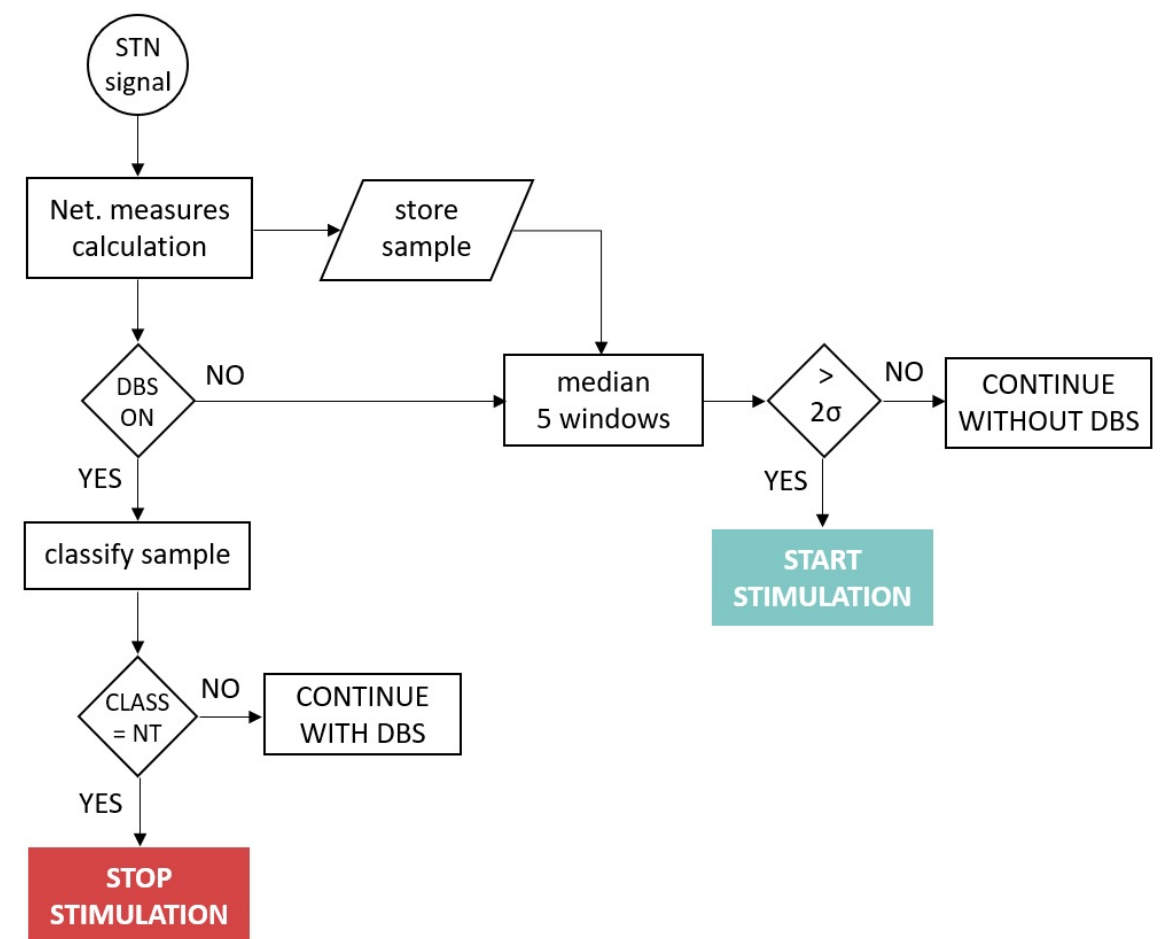

Figure 7.4: Flow diagram of the system operating mode 
Shut down the stimulation . In this usage scenario, the system is stimulating (DBS ON), and it has to decide whether to stop the stimulation. i.e., the system is registering $T$ samples (subthalamic signal associated with the tremor regarding the extracted features), but at a certain point begins to register NT samples (subthalamic signal associated with atremorous state regarding the extracted features). Sensitivity here is the ability of the system to classify a sample as $T$ correctly. While specificity is the ability to classify a sample as NT correctly. A false positive in this scenario represents that the system classifies an arriving sample as $N T$ being $T$. While a false negative represents that an arriving sample is classified as $T$ being NT.

The SVM module has been trained to discriminate these two types of samples. Its performance is presented in table 7.1. In this scenario, we want the system to have a high degree of specificity and a low percentage of FPR.

In the case of patient 2, there is a specificity of $100 \%$ and an FPR of 0 . This is the ideal case. $100 \%$ of the NT samples are identified without failure. Nevertheless, in the case of patient 3, there is a 90.8 specificity and an FPR of 9.2. This means that about 9 out of 10 NT samples are evaluated correctly, but 1 out of 10 have been incorrectly classified as tremor.

Notice that the implication of an FPR $\neq 0$ has on this scenario: If the window being evaluated is incorrectly classified as tremorous, the system will continue stimulating, and it will evaluate the next window. Continue stimulating has no effect on the symptomatology and goes unnoticed for the patient (as represented in 7.3-b2.). We are interested in having high specificity, but the fact of not reaching $100 \%$ is not critical.

Start up the stimulation . Contrary to the previous use case, in this usage scenario, the system is in standby, sensing. For each incoming window, the system must decide whether to start stimulation. i.e, the system is recording $N T$ samples, but at a certain point begins to register TO samples.

Sensitivity here is the ability of the system to classify a sample as NT correctly. While specificity is the aptitude of correctly classify a sample as TO. A false positive in this scenario represents that the system classifies an arriving sample as TO being $N T$. While a false negative represents that an arriving sample is classified as $N T$ being TO. Likewise, we want the system to return high values of specificity and a low percentage of FPR.

The proposed system bases the detection of TO on the existence of a non-linearity peak above $2 \sigma$, as described in section 5 . The reasons we opted for this solution are:

- A peak above $3 \sigma$ is detected within the TO section of all subjects (specificity = $100 \%$ ), indicating a clear pattern of sudden non-linearity increase in the neuronal signal of the subthalamic nucleus, just before the patient experiences physical tremor. This peak can be used as a trigger for the decision to begin stimulation by the system. It is a simple and effective system. 


\begin{tabular}{|c|c|c|c|c|c|}
\hline File & ACC & Sensitivity & Specificity & FPR & FNR \\
\hline 1 & 84.8 & 89.83 & 79.24 & 20.75 & 10.17 \\
\hline 2 & 98.4 & 97.44 & 100 & 0 & 2.56 \\
\hline 3 & 94.3 & 95.20 & 90.8 & 9.2 & 4.79 \\
\hline 4 & 90.3 & 87.13 & 92.05 & 7.94 & 12.86 \\
\hline
\end{tabular}

Table 7.1: SVM-Subsystem performance in stopping the stimulation

\begin{tabular}{|c|c|c|c|c|c|}
\hline File & ACC & Sensitivity & Specificity & FPR & FNR \\
\hline 1 & 69.6 & 74.54 & 50 & 50 & 25.45 \\
\hline 2 & 77.1 & 60 & 81.57 & 18.42 & 40 \\
\hline 3 & 86.7 & 80 & 92.06 & 7.93 & 20 \\
\hline 4 & 82.1 & 81.39 & 84 & 16 & 18.6 \\
\hline
\end{tabular}

Table 7.2: SVM-Subsystem performance in starting the stimulation

Notice that despite detecting a peak above $3 \sigma$ in all recordings, a conservative threshold has been set at $2 \sigma$ (statistical significance of the peak $\mathrm{p}<0.05$ ) in order to ensure that the peak triggers the start of stimulation in unseen futures cases, which might perhaps present a less significant peak.

- An SVM system has been trained to distinguish NT samples from TO, obtaining worse results than in the previous usage scenario. This was the expected outcome since the classes to be classified are more similar between them. Remember in this regard that TO is a transition state between NT and TO. Results are presented in table 7.2. As can be seen, the specificity does not reach $100 \%$ in any of the patients, obtaining higher values of FPR the previous use case. With the addition that in this case, the importance of correctly classifying a sample is more critical than in the previous usage case. If the window being evaluated is incorrectly classified as $N T$, being a TO sample, the system will continue in standby, not starting the stimulation. As soon as the patient leaves the TO state and enters the $T$ state, he or she will begin to tremble (as represented in figure 7.3-a1. It is crucial that the system does not leave the patient without stimulation, needing it. This is a red line for the system.

\subsection{Discussion}

\section{Preferred network measures}

As discussed above, the three network measures studied show similar behaviour. However, our results show that the clustering measure detects more abrupt changes in the non-linearity level of the subthalamic signal, which translates into more marked peaks. Thus, we would implement the trigger for start up the DBS based on the clustering peaks. 


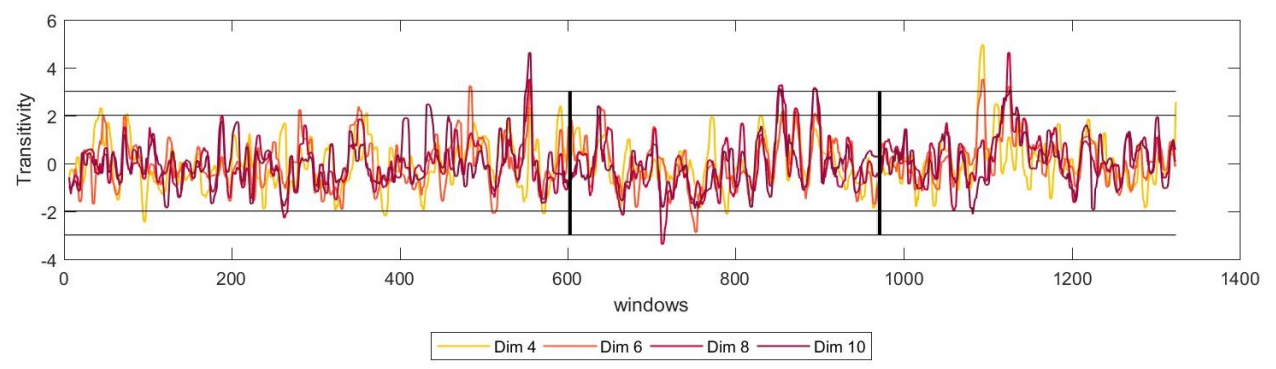

Figure 7.5: Moving window $\varepsilon$-recurrence network analysis showing the median moving average of transitivity, before during and after the start of the tremor. The temporal profile of the measure is shown for different values of $m=4,6,8$ and 10 . The vertical line represent the time at which the patients transit from NT to TO (left) and from TO to $T$ (right). The horizontal lines represent the \pm 2 and \pm 3 standard deviation, the thresholds for statistical significance

\section{Setting of the system parameters}

Since the actual mechanism of action of DBS is still unknown, there is not a standardised process to fix the stimulation parameters (rate, pulse width and voltage), which have to be fixed by the medical staff at the time of its implantation. The clinicians can later change these parameters during patient visits to the hospital to maximise the clinical improvement of the symptoms.

In order to use the proposed closed-loop DBS system, it will be necessary to calculate the parameters $\tau$ and $m$ for each subject. There are two possibilities: 1) They can be fixed, as with the remainder of the parameters, at the time of implantation and reconsidered during the subsequent visits to the doctor, or 2) they can be calculated from each window data in real time.

This decision will have to be taken in the design phase of the IMD. Whereas the second option is more accurate, in so far as the value of the parameters is data-driven and performed in real time, also entails a higher computation. This is inconvenient because neurostimulators, as with any other IMD, have restricted capabilities of energy, storage, and computing power [149].

Considering these restrictions, I would lean towards the first option. In this case, we need to test the robustness of the proposed method against the parameters $\tau$ and $m$. It is necessary to know to what extent these parameters affect the detection of the tremor states, and transitively the detection of the tremor, i.e., the robustness of the proposed approach.

For that matter, we have studied the dynamics of the system for several numbers of dimensions, $m$, of the reconstructed phase space. The results show that for all the possible number of dimensions, the network measures show a similar trend, stabilising for higher values of $m$. Figure 7.5 shows these results for transitivity for one of the patients.

From these results, it can be concluded that, even if the number of dimensions necessary to reconstruct the phase state change slightly, this would not affect the performance in the detection of the tremor. Nevertheless, more studies in this 
direction would be necessary.

For its part, the value of $\tau$ across windows has a very low variance, oscillating in a unit. For example, given a patient for which the optimal value of $\tau$ for most of the windows is $\tau=4$, we could find some windows of this same patient with an optimal value of $\tau=3$. In this case, we would set $\tau=4$.

Nevertheless, it have been found that the performance of the system is suitable across all the windows, and there is not an appreciable effect on the prediction of the peak before the onset of the tremor. Hence, we can conclude that the system is robust against the choice of the parameter $\tau$, at least in our case, in which this parameter has a minimal variance.

\subsection{Conclusions}

The behaviour of the STN becomes highly non-linear during tremor episodes, when compared with the basal state (NT), making the geometry of the phase state more structured. We hypothesise that the witnessed increase in non-linearity, as reflected by the shift in the network measures, could be attributed to the change in synchronization between the neurons during the tremor episode, as seen in a previous study [133].

$\varepsilon$-recurrence network analysis is a suitable method to distinguish the transitions between movement conditions. Furthermore, the implemented method has the advantage of being able to deal with both short and non-stationary data, making it a good option for LFP data. These two facts make this procedure appropriate for its application to a closed-loop DBS system.

The setting of the parameters of the system, $\tau$ and $m$, can be taken at the time of the start-up of the device and adapted if necessary, during the visits of the patient to the clinician. This solution takes into consideration the inherent constraints of the IMD: energy, storage and computing power, making viable the implementation of the proposed solution.

Given the difficulty of getting STN recordings like the used at this work, only four patients have been studied. Despite having found very similar results in all of them, it would be necessary to consolidate these results in more patients. Our intention with this work is to propose that $\varepsilon$-recurrence networks may be a useful tool in the design of systems that interact with brain signals, not only in PD $[136,137]$ since all neural activity is a source of non-linear, non-stationary data. 



\section{8}

\section{Closed-loop deep brain stimulation based on a stream-clustering system}

Here, a Closed-loop DBS system for PD is presented, which is able to recognize, with 100\% accuracy, when the patient is going to enter into the tremor phase, thus allowing the device to stimulate only in such cases. The system has been designed and implemented within the data stream mining paradigm, suitable for our scenario since it can cope with continuous data of a theoretical infinite length and with a certain variability, which uses the synchronization among the neural population within the Sub Thalamic Nucleus as the continuous data stream input to the system.

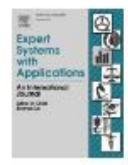

Closed-loop deep brain stimulation based on a stream-clustering system

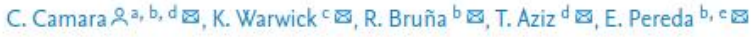


eurostimulators deal with infinite and non-stationary signals such as LFP. The supported system needs the intelligence to adapt itself to changes and provide the most appropriate treatment in each moment. In this demanding scenario, DSM emerges as a promising technique to deal with this sort of restrictions. To the best of our knowledge, none of the existing solutions uses LFP signals as data streams. Therefore, I take advantage of the full potential of DSM and have designed a closedloop DBS system using LFP streams.

In the first part of the work, it is studied whether the patterns of LFP connectivity within the STN change when the motor symptoms of PD emerge. As suggested by the results, the tremor onset implies a change in connectivity in some frequency bands, which can be used to improve the DBS systems currently employed. Our results also show the potential of STN-LFP synchronization streams for closed-loop DBS purposes. In fact, the behaviour of the clustering, which is the core of the system, is remarkable, achieving an accuracy of $100 \%$ in all cases. The system has demonstrated that it is able to detect concept drifts in the data by clustering the arriving instances correctly with a high level of immediacy.

The results are promising since, to the best of our knowledge, this is the first study attaining such levels of accuracy. Nevertheless, as future work, it would be ideal for reproducing them in new datasets with more patients, when available. I hope this contribution can serve as a seed to future work that explores the use of STNLFP synchronization for closed-loop DBS. In addition, and given the accuracy levels achieved here, it would also be interesting to study if data stream mining algorithms respond well in other on-demand stimulation scenarios or in other actuating devices in the medical environment.

\subsection{Neural oscillations versus synchronization}

One of the interesting features we can explore using the LFP is the neural oscillations, whose deviation from the typical patterns of healthy brain activity is often an indication of a pathological condition. Such deviations may not be restricted to the modification of, e.g., the spectral power of the individual LFPs in the frequency domain, but may include alterations in the communication between neural populations, that is, their functional and effective connectivity [150]. Indeed, the relation between local synchronization, as reflected by the spectral power at certain frequency bands, and connectivity among neurons, remains an open question. Some dopaminergic studies have suggested that the pathological performance of the STN, in which neurons oscillate synchronously with high amplitude, also involves an increase in spatial synchronization. When neurons trigger at the same time, there is no temporal delay, which is necessary for the communication between neurons, that is, functional and/or effective connectivity [132]. Thus, in these studies, it has been observed that during ON medication periods, the pathological spatial synchronization disappears, and connectivity between STN neurons is increased. 


\section{LFP-STN Neural Oscillations}

There are many studies on local synchronization. It is well known that when neurons oscillate synchronously in the basal ganglia, mainly in the subthalamus, it entails dysfunctional motor states in PD patients. This has been observed at the single-cell [151] and at the LFP level [131, 152]. Most of these studies found excessive neuronal synchronization in the beta frequency band (between 12 and 30 $\mathrm{Hz})$, which is linked to bradykinesia and rigidity $[131,153]$. Additionally, this beta synchronization is linearly related to the degree of levodopa administered [130] and to the treatment of PD using DBS [154-156].

However, the influence that beta-band synchronization has on the occurrence of the resting tremor is still an open question. Indeed, some authors claim that there is no relationship between these events [157], while others maintain the contrary [158]. Additionally, it has been observed that there is no causal link between the dopamine deficit in the striatum and the severity of the tremor [159].

There is also controversy as to whether this beta-band synchronization is generated within the STN or if it merely reflects the overall synchronization in the basal ganglia circuit $[152,160]$. In this regard, there are in-depth studies that conclude that these oscillatory patterns, at least in the beta band, are generated within the STN [161].

\section{LFP-STN Synchronization}

There are not many studies on functional connectivity in STN-LFP, whose analysis I believe is essential not only to understand the functioning of the basal ganglia itself but also to improve the treatments of some extended neurological diseases, such as PD [94]. It has been observed that, as in the case of neural oscillations, connectivity in the beta band is modulated by levodopa [132]. Yet to the best of our knowledge, there is no published research that studies synchronisation within the STN during both tremorous and atremorous states.

Besides, a drawback of many studies is that they consider only the beta band. Yet in $[162,163]$, the authors found different behaviours at the lower $(<20 \mathrm{~Hz})$ and upper $(>20 \mathrm{~Hz})$ beta bands. This result suggests that the oscillations take place only in the lower beta band, and what is observed in the upper beta is just a "contamination" of these activities. In order to be as thorough as possible, in this work I estimated the synchronisation levels during tremor and atremorous episodes, not only in the lower $([12-20] \mathrm{Hz})$ and upper $([20-30] \mathrm{Hz})$ beta bands, but also in the tremor $([3-7]$ $\mathrm{Hz})$, alfa $([8-12] \mathrm{Hz})$ and gamma $([30-45] \mathrm{Hz})$ bands.

Given the heterogeneity across subjects, and to be certain that the results are valid, I performed the analysis per subject. This allows us not only to deal with intersubject heterogeneity, but also to observe, in a subject-specific way, what happens to the synchronisation level in the STN before, during, and after the appearance of the tremor. 
The purpose of this study is twofold. Firstly, I want to characterise synchronisation in the STN, and study which of them are more informative differentiating the distinct states. To this end, I apply both functional and effective connectivity methods. Moreover, and given that it remains an open question, I want to know which frequency bands show changes in the synchronisation with the appearance of the tremor. Secondly, I face the design of a data stream mining system which is able to identify the dynamical states in which the patient transits, to recognise and adapt itself to concept drifts, to perform in real-time and to do it whilst employing a limited amount of resources (memory).

\subsection{Signal Preprocessing}

In this study, the transition subset of the dataset is used, as indicated in section 4.1. It consists of four files that collect neural information while the patient transits from $N T$ to $T O$.

LFP data was two-pass filtered into tremor, alfa, lower-beta, upper-beta and gamma bands using band-pass filtering with a 500 order FIR filter designed with Hamming window, and using 2 seconds of real data as padding. The movement artefacts around $1 \mathrm{~Hz}$ and line noise (in Europe, $50 \mathrm{~Hz}$ ) were excluded after this filtering step. Some of the connectivity methods work with the analytic signal. To this end, the Hilbert transform of the filtered LFP data was performed.

Finally, the data was segmented in windows containing ten cycles at the central frequency of the band, with an overlap of $50 \%$. The level of overlapping is motivated for capture with high temporal resolution the rich, dynamic behaviour that STN-LFP has.

The EMG signal in tremor is made up of bursts, whose peaks are at a frequency of $30 \mathrm{~Hz}$ and above. Consequently, EMG data were high-pass filtered above $30 \mathrm{~Hz}$ using a two-pass procedure with a 500 order FIR filter designed using a Hamming window. The EMG signal was then rectified using the Hilbert envelope. This rectified signal is filtered again using a FIR 2-45 Hz filter. With this, the tremor in Hilbert's rectified signal is already at low frequencies.

\subsection{Synchronization analysis}

In this work, I applied two classical linear methods (Coherence and Amplitude Envelope Correlation), two information-theory based methods (Mutual Information and Phase Transfer Entropy), a phase synchronisation method (Phase-Locking Value) and a high-order spectra method (Bicoherence). All of them are FC measures, except the phase transfer entropy, which belongs to EC methods. Their mathematical description can be seen in 2.3.2. These measures have been applied after filtering the data to the bands of interest: Tremor, Alfa, Lower Beta, Upper Beta and Gamma bands separately. By applying this battery of measures, I intended to thoroughly characterise both linear and nonlinear connectivity within the LFP of the STN in order to have a detailed description of the pattern of FC/EC within the STN. 


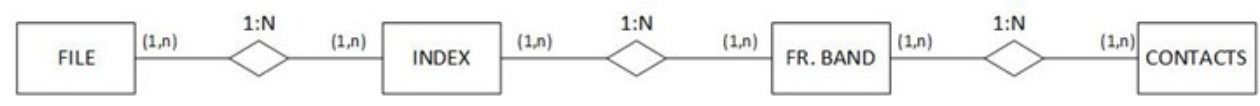

Figure 8.1: E-R Model. The E-R diagram represents the relevant entities of our dataset and their interrelationships.

Henceforth, I briefly describe these indexes.

The entity-relation diagram of figure 8.1 illustrates the dimensions of information in our dataset. We have information on what is happening within the STN between each pair of contacts, at each frequency band, and from the perspective of each connectivity index. This constitutes a lot of information, but certainly, not all of it is useful in identifying the movement states. We, therefore, need to reduce the dimensionality of the data, ignoring in the subsequent steps the irrelevant attributes. This step is important for several reasons: Firstly, to improve the performance of our task, since subjoining irrelevant features is self-defeating for ML algorithms. Secondly, to reduce the computational requirements of the system, since fewer features need to be calculated by the IPG in real-time. Finally, it allows us to improve the explicability of our results, thereby gaining insight into the FC mechanisms within the STN associated with the appearance of the tremor.

To this end, we could simply perform a manual selection of the attributes, by removing or adding them one by one and testing the results, but this is a naïve and arduous process. Methods from ML are, however, very useful. In this work, I used a wrapper method with a KNN classifier with $k=5$ performing ten-fold crossvalidation for the attribute selection problem. $\mathrm{KNN}$ is a lazy and non-parametric algorithm, which is highly sensitive to irrelevant attributes. Although wrapper methods are computationally more expensive than filter methods, they render better performance. Feature selection is calculated here in order to unveil which synchronization indexes and frequency bands are more informative. This process will not be carried out by the IMD, and it is not part of the proposed clustering system.

I applied the feature selection procedure in two steps. Firstly, I studied the preferred frequency bands, those that produce the highest accuracy. Subsequently, I performed a second round of feature selection in these bands. I have summarised the selected bands and features in table 8.1.

The preferred bands in all cases were the tremor (TB) and the lower-beta (LB) bands. The selected indexes per each band were the same in all the cases, except for the phase indexes, PLV and PhTE which are not always selected per all the recordings. However, the degree of accuracy varied across files, so we cannot conclude at this point that only one index is valid, but we would choose three for further analysis. 


\begin{tabular}{|c|c|c|}
\hline FILE & $\begin{array}{c}\text { SELECTED BANDS } \\
\text { (Accuracy) }\end{array}$ & $\begin{array}{c}\text { SELECTED INDEXES } \\
\text { (Accuracy) }\end{array}$ \\
\hline \multirow[t]{2}{*}{1} & TB: $96.56 \%$ & $\begin{array}{c}\text { PhTE: } 93.78 \% \\
\text { BiCOH: } 88.15 \% \\
\text { MI: } 87.02 \%\end{array}$ \\
\hline & LB: $91.43 \%$ & $\begin{array}{l}\text { BiCOH: } 81.31 \% \\
\text { PhTE: } 75.57 \% \\
\text { MI: } 75.95 \%\end{array}$ \\
\hline \multirow[t]{2}{*}{2} & TB: 98.35\% & $\begin{array}{c}\text { PhTE: } 88.75 \% \\
\text { BiCOH: } 86.89 \% \\
\text { MI: } 82.10 \% \\
\text { PLV: } 75.50 \%\end{array}$ \\
\hline & LB: $92.99 \%$ & $\begin{array}{c}\text { MI: } 80.09 \% \\
\text { BiCOH: } 78.86 \% \\
\text { PhTE: } 72.05 \% \\
\text { PLV: } 70.95 \%\end{array}$ \\
\hline \multirow[t]{2}{*}{3} & TB: 93.13\% & $\begin{array}{l}\text { BiCOH: } 76.13 \% \\
\text { MI: } 66.63 \% \\
\text { PhTE: } 61.50 \%\end{array}$ \\
\hline & LB: $87.15 \%$ & $\begin{array}{c}\text { BICOH : } 76.13 \% \\
\text { MI: } 66.63 \% \\
\text { PhTE : } 61.50 \%\end{array}$ \\
\hline \multirow[t]{2}{*}{4} & TB: $93.15 \%$ & $\begin{array}{l}\text { BiCOH: } 79.42 \% \\
\text { MI: } 64.39 \%\end{array}$ \\
\hline & LB: $85.87 \%$ & $\begin{array}{l}\text { BiCOH: } 67.99 \% \\
\text { MI: } 57.31 \%\end{array}$ \\
\hline
\end{tabular}

Table 8.1: Features Selection results using a wrapper method with a K-NN algorithm.

measures, each of them calculated per each of the four evaluated frequency bands. After that, I reduced the dimensionality, choosing only two frequency bands (TB and LB) and only three indexes, PhTE, MI and BiCOH. Hereinafter I continue our experimentation only with these selected features; only these will be evaluated in our system.

\subsection{Data Stream Mining}

Data mining refers to the set of technologies to handle larger datasets to find patterns, trends or rules and explain data behaviour [164]. In classical data mining techniques, a model is built during the so-called training phase, in order to make future predictions, recognising the class of a given sample not seen by the system before - the so-called testing phase. These technologies have consolidated due to the huge amount of data, which is collected and handled on a day to day basis. Indeed, this is a trend that continues growing at a fast pace in different areas, especially in the healthcare context [165].

However, this approach does not always fit well in real-time analytic scenarios, in which it is important to analyse the data, extract the relevant features and take decisions in real-time. In other words, while data mining can handle well considerable quantities of data, it does not consider the continuous supply of data. Models cannot be updated when new information arrives. This implies that they are not able to self-adapt in response to the observation of new samples, and the complete training process has to be repeated. Furthermore, the length of the data feed is much larger than the storage -for instance, our scenario in which we have a neural 
signal monitored during the entire life of an individual.

Contrary to traditional data mining, data stream mining (DSM) involves a set of algorithms, emerged as a paradigm to address the continuous data problem and changes in the behaviour of the stream. A data stream can be considered a signal with time as an independent variable, which needs to be processed online, as each sample arrives. In this way, data samples of such signals will feed the model sequentially, training it in a continuous way. In such systems, we do not distinguish between the training and the testing phase, as both tasks are continuously updating the model online. This way of functioning is very interesting since it allows the system to adapt itself to changes in the data, as we will discuss hereinafter.

There are, though, two main algorithmic challenges in this scenario. First, the data stream has infinite length and arrives fast, and therefore it is necessary to extract information from it in real-time. Second, the data may be evolving, experiencing shifts (non-stationary data such as neurological signals) and models must adapt themselves when there are changes in the data [166].

The core assumption is that training samples arrive very fast and should be processed and discarded to make room for new samples, thus being processed one time only. More specifically, DSM presents a set of different requirements [166]:

- Uniqueness. Each sample must be processed before a new one arrives, and it has to be done only once, without the retrieval of any previous samples being possible. Since our source of data is the STN, our data stream will be endless. This fact makes this requirement critical for our purpose.

- Limitation of resources. The hardware that will analyse the data, the IPG, like any other IMD, has restricted capabilities of energy, storage, and computing power [149], principally due to its implantation. This limitation of memory is one of the main motivations behind using data streams, as memory can be overloaded when too much data is stored in it.

- Limitation of time. DSM algorithms should scale linearly with the number of samples to work in a limited amount of time. The algorithm should be able to process the data at the speed of the stream. In the proposed system, the algorithm has to test and train at the speed of the neurostimulator register and pre-process a time window, with the minimum plausible delay to detect the shift in the tremor state as soon it appears.

- Evolving time series. DMS works with non-stochastic time series, which evolve over time. The changes in the data are called concept drifts, and our DSM algorithm has to able to detect them, adapting the output.

- Readiness. The algorithm must be able to commence working after a learning period as small as possible. 
- Immediacy. An algorithm should be ready to produce the best model at any time, regardless of the number of processed samples.

\subsection{Stream Clustering}

The objective of our system is to detect the tremor through the data stream recorded from the electrodes. In this scenario, the labels of the new samples are unknown to the system; in fact, its purpose is to create them. We are, therefore, in an unsupervised learning scenario. The objective of stream clustering is to cluster the samples of the time series according to the speed at which the data stream is generated, updating the clustering with each newly arriving sample, and within the constraints presented in the previous section.

As with classical clustering techniques, the main goal of stream clustering is grouping the instances into clusters according to their commonalities, so that instances within each cluster are similar to each other while instances from different clusters are distinct. Obviously, the success in this task is closely related to the quality of our features, in our case the value of the synchronization indexes. This is one of the reasons why a feature selection was applied prior to the analysis with DSM.

Here we use an ad-hoc algorithm for DSM, ClusterGenerator, since it ensures an evaluation unbiased by possible incorrect outcomes of stream clustering algorithms [167]. We briefly describe it now.

The algorithm forms and continuously maintains microclusters, a technique used in other stream-clustering approaches. A microcluster is a compact representation of the data distribution, from which we can derive the mean and the standard deviation. It is represented as a cluster feature tuple $C F=(n, L S, S S)$, where the data stored in it are the number of points within the microcluster $n$, their linear sum $L S$, and their squared sum SS. Microclusters are representatives for sets of similar data points.

Stream clustering algorithms usually alternate between two phases, commonly called online and offline phases. In the online phase, microclusters are created. Working with microclusters is a key point since in this way, it is not necessary to access the past samples of the stream, i.e each sample will be accessed only once, which satisfies the uniqueness condition. During the offline phase, time is not critical and is typically used for the user to make an analysis of the existing clusters at each temporal moment, stored in the so-called snapshots. This phase typically requires more than one pass to the stored data (to the microclusters) and is a very useful phase in big data analytics environments. Cluster Generator adopts only the online phase, which assures us a lower computation requirement, which is a very important property in our domain. In addition, the offline phase usually reduces the number of microclusters. However, in the use scenario of the presented system, the number of microclusters is small and determined by the clinical problem, so that offline clustering is omitted. 


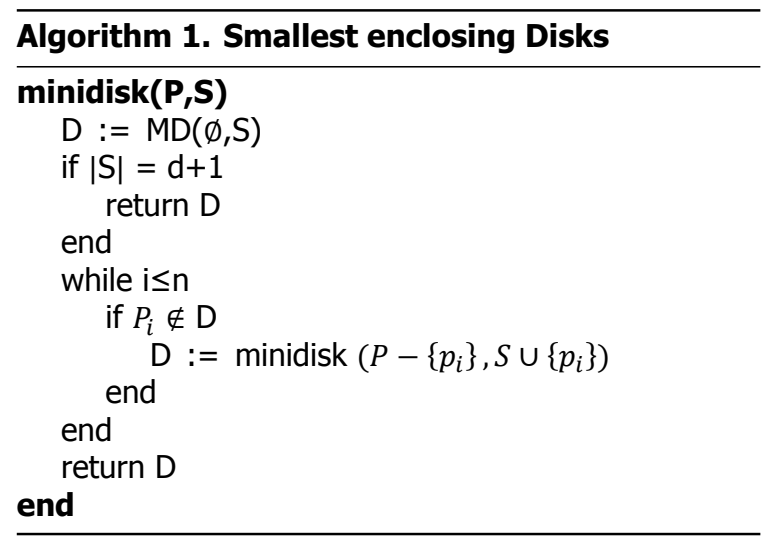

Cluster generator is a partitioning-based clustering algorithm. The cluster boundaries are determined as described in $[168,169]$, that suggest creating spherical clusters of the smallest possible radius that contains all the instances of the cluster. The pseudocode of this algorithm is presented in Algorithm 1 and described hereinbelow. For further details, the source code of the used algorithm is publicly available at [170].

Given a set of points $P=\left\{p_{1}, \ldots, p_{n}\right\} \subseteq \mathbb{R}^{d}$, let $\mathrm{D}(P)$ denote the closest disk of smallest radius that contains $P . \mathrm{D}(P)$ exists and it is unique. For $P, S \subseteq \mathbb{R}^{d}, P \cap S=\emptyset$, let $\operatorname{MD}(P, S)$ be the smallest disk containing $P$, with all the points in $S$ on its boundary. So we have $\operatorname{MD}(P, \emptyset)=\mathrm{D}(P)$, and $\operatorname{MD}(\varnothing, S)$ to be the smallest disk containing all the points of $S$ on the boundary. $D(P)$ is determined by at least tree points on its boundary. i.e there is a subset $S$ of $P$ on the boundary of $D(P)$ such that $S \leq 3$ and $D(P)=D(S)$; so if $D(P-\{p\}) \neq D(P)$, then $p \in S$, and $p$ lies on the boundary of $D(P)[168]$.

minidisk is a recursive algorithm that computes $D(P, S)$ incrementally by adding the points in $P$ successively while maintaining the smallest enclosing disk. When the algorithm calls minidisk $(P, \emptyset)$, all sets $S$ that resulting from recursive calls are affinely independent [169]

Summarising, the reasons why I opted for DSM are as follows:

- Neural activity is a source of non-linear, non-stationary data. Thus, it calls for a system able to adapt itself, learning in a continuous way.

- IMDs are devices with restricted capabilities of energy, storage, and computing power. Any proposed measure to operate imbibed in them should be as efficient as possible. DSM methods are thought to obtain the maximum accuracy with minimum time and memory use [166]. 


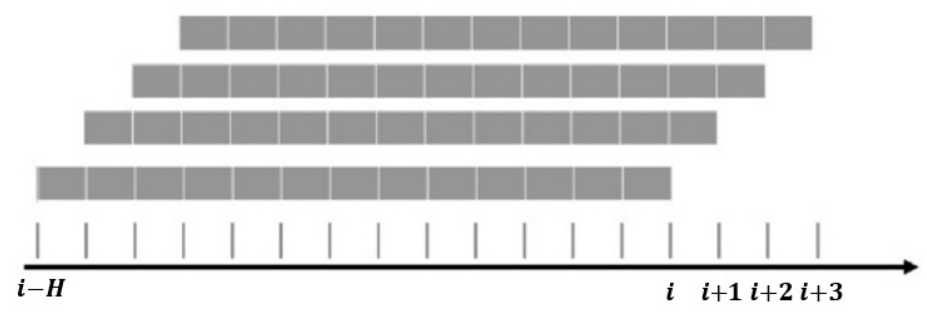

Figure 8.2: Example of sliding window strategy

- It has been applied to other problems dealing with sensor data and the Internet of Things, due to its capacity to monitor processes and improve their quality $[166,171]$

- The main motivation for choosing a stream-clustering technique, instead of a classification one, is determined by the context of the application of the system: a clinical environment during a neurosurgery procedure. In this scenario, the best of systems that could be designed is one that would be able to recognise the different movement states autonomously with a high level of accuracy. This is due to the short time available in the clinical environment for a possible training of the system. Note that the system must be prepared for full operation during the peri-operative period. For this reason, the main objective is to design a system capable of separating the samples that arrive in real-time, without the need for prior training or data labelling. With a stream-clustering system, we only need to expose the patient to the different movement states, so that the clusters are open. This process is faster and more efficient than collecting long periods of subthalamic signal, then labelling them and training a classification system. This fact is key to the usability of the system in a real clinical environment.

\subsection{System Description}

One of the relevant aspects of the system is how data is treated. In a real-time application, in which neural samples arrive continuously to the system in a nonpredefined order, an on-line analysis was used to evaluate LFP streams following a sliding window strategy, in which the size of the window was fixed and the buffer kept the newest instances. Similar to the first-in, first-out data structures [172], whenever a new instance was inserted into the window, another instance $i-H$ was forgotten -, where $H$ represents the window size (see Figure 8.2).

In clinical application, the system starts up during the peri-operative period. The IPG starts registering the STN-LFP signals, which are divided into 10-cycles windows. After that, the synchronization index is calculated over each LFP window and per each contact pair, obtaining a set of coefficients: 


$$
S=I(L F P)=\left\{I(L F P)_{w=1}, I(L F P)_{w=2}, \cdots,(L F P)_{w=n}\right\}
$$

where $I(L F P)_{w=i}$ is:

$$
I(L F P)_{w=i}=S_{w(i)}=I_{w=i}^{c_{1}-c_{2}}, I_{w=i}^{c_{1}-c_{3}}, I_{w=i}^{c_{2}-c_{3}}
$$

where $w$ is the number of the observed window, $c_{x}$ and $c_{y}$ are the channel pairs between which the synchronisation is calculated, $I$ is the synchronisation measure employed and $S$ represent the result of the synchronisation per each window, i.e our datastream. Finally, the LFP stream $S$ is sent to the clustering algorithm.

According to Algorithm 2, the system works in the following way. Firstly, during the set-up phase, the necessary clusters are initialised inside the system. For that, the system observes the activity of the STN for some time, during which it is necessary for the patient to experience the different movement states. During this time, the signals on each electrode are collected, pre-processed and the synchronisation measure is extracted. The output of the synchronisation index determines autonomously which cluster the sample will go to. The process finishes when three clusters (NT, TO and T) are created, one per movement condition.

Once the clusters are initialised, the system enters into the operation phase, whose core is very similar to the set-up phase. The main difference is that in this phase, the clusters are already created. The system should not open new clusters, but merely evaluate the arriving samples, decide the cluster each sample should go to and update the microcluster of the elected cluster after the instance is included. I will later discuss the evaluation of this process. The operation phase of the system is depicted in Figure 8.3.

\subsection{Experimental Analysis \\ Clustering-Streams Results \\ Evaluation Measures}

Before presenting the results, I describe the evaluation measures. I employed external measures since the ground truth is available against which to compare the clustering result.

Accuracy (ACC): Fractions of instances assigned to their correct cluster.

$$
a c c=\frac{\sum_{i=1}^{N_{w}} T P}{N w}
$$

where TP means a true positive (an instance correctly identified), and $N_{w}$ is the total number of windows in the record.

Cluster Mapping Measure (CMM): CMM is an ad-hoc measure for stream clustering. Similar to accuracy, it quantifies how a given clustering is different from the 

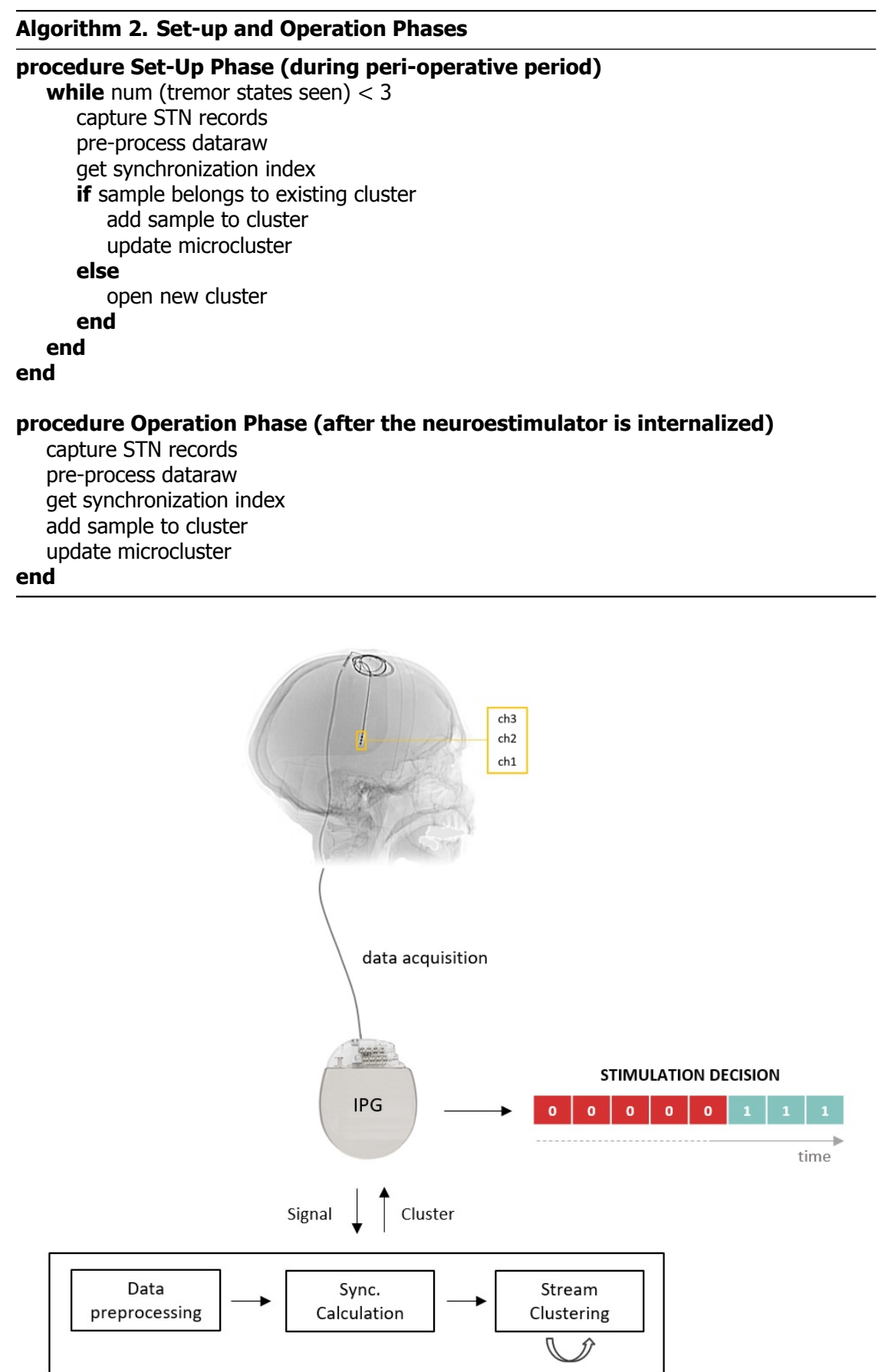

Figure 8.3: Stream Clustering - Closed-loop DBS system model 
ground truth, but in this case, taking into account the type of errors that can occur during stream clustering, called faults. These faults can be derived from missed, misplaced or noisy points - the estimated CMM ranges between 0 and 1 . The lower the faults, so the closer CMM is to 1 [167].

\section{Experimental Analysis}

In order to follow this section, I first introduce the concepts of weight and horizon [167]:

Weight. Consider $t_{i}$ (the time in which the sample $S_{w(i)}$ arrived to the system) and $t_{0}$ (the present time), with $t_{i}<t_{0}$. The weight of $S_{w(i)}$ is the decay function: weight $\left(S_{w(i)}\right)=2^{-\lambda\left(t_{0}-t_{i}\right)}$, where $\lambda$ is a parameter that controls the ageing of the function. In this case, $1 / \lambda$ is the half-life of $S_{w(i)}$. In our case, $\lambda$ is set to zero, so I do not consider decay in our stream.

Horizon. Since this paradigm works with real-time and infinite data, stream clustering techniques have to forget past samples. To this end, only a subset of the recent samples of the stream $\mathrm{S}$ is considered at a given time. The horizon $(H)$ for a stream $S$ and a defined threshold value of $\xi$, is defined as $H=\left\{S_{w(i)} \in S \mid W\left(S_{w(i)}\right) \geq \xi\right\}$.

The recordings capture the neural activity of patients while transitioning from NT to $\mathrm{T}$. Thus, the sequence order the recordings is $N T \rightarrow T O \rightarrow T$, as shown in figure 8.4a. If we evaluate the system over these records, we can test if the system can adapt to concept drifts, detecting the three states, and thus opening the three clusters. However, this would be similar to training the system, without testing it later (Although in stream clustering we do not talk of training and testing, we will refer to it this way for the sake of clarity), since with this experimentation it is not possible to test immediacy and readiness, essential features to indicate if the system will work properly in real-time and real environment states. We need to test the system once the clusters are opened. To this end, as stated in Algorithm 2, we firstly expose the system to the tremor states (set-up phase), to later test states (operation-phase) to see if it is able to identify the arriving instances correctly, by adding them to the correct cluster.

To this end, the files were reordered, positioning a subsection of windows of each state at the beginning of the file, as shown in figure 8.4b and 8.4c. The number of selected windows is $50 \%$ of the smallest section in the file. We used this subsection as a training period, in which the system was exposed to all the tremor states. Accordingly, a cluster of each type is opened. The horizon, in this case, was fixed to the number of windows within this subsection ( $3 x$ in the example of the figure).

It is important to remark here that this period is just to simulate the functioning of the system in a real environment. It is not a typical training phase itself since it is the system itself that opens the clusters based on the instances it receives; the label information is not provided. The sequence order of the files to carry out the 
a)

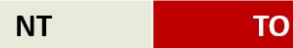

\begin{tabular}{l|l} 
TO & T \\
\hline
\end{tabular}

$\mathbf{T}$

b)

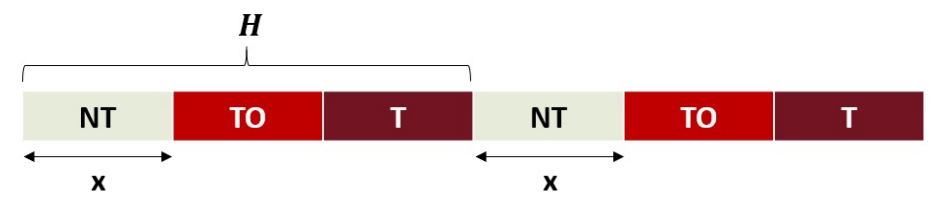

c)

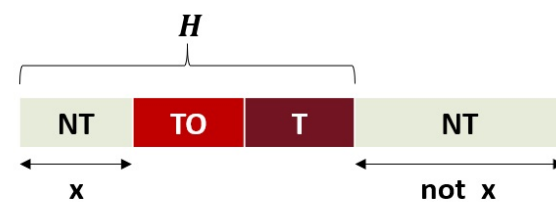

Figure 8.4: a) Original order of the states in the recordings. b) States re-ordered to test OP-2: in which initialised clusters during peri-operative period will be maintain in the system memory. I simulate that by making the section of the operation phase as long as sections of set-up phase c) States re-ordered to test OP-1: in which initialised clusters during peri-operative period are not maintained in the system memory. I simulate that by making the sections of the operation phase longer than sections of set-up phase

experimental analysis is therefore $N T \rightarrow T O \rightarrow T \rightarrow N T \rightarrow T O \rightarrow T$.

At this point, the issue of forgetfulness becomes important. To assess the influence of whether maintaining the opened clusters or not, I designed two possible configurations for the operation phase: OP-1, corresponding with Figure 8.4c, in which I provoked the system to forget some of the clusters by fixing the parameter $\mathrm{H}$ low enough; and OP-2, corresponding with Figure $8.4 \mathrm{~b}$, where the opened clusters are not forgotten. The results for both configurations are presented in Table 8.2.

In the OP-1, the system reaches the so-called point of forgetfulness. An example is depicted in Figure 8.5, in which the cluster $T$ has been forgotten. Under this situation, if the arriving points are not strongly separable from the existing ones, they would not be assigned to their proper class. I have tested this, and have found that this error never happened when comparing a sample of $T O$ or $T$ with a sample of $N T$. HoIver, I have found that it is possible for the system to get confused between $T O$ and $T$ instances if one of these clusters is forgotten.

Actually, this would not suppose a problem in the particular case of our system, since both clusters (TO-cluster and T-cluster) will produce the same output, since the decision as to whether to apply stimulating pulses or not in both cases would be positive. In fact, I could perfectly have merged these two states. However, I 


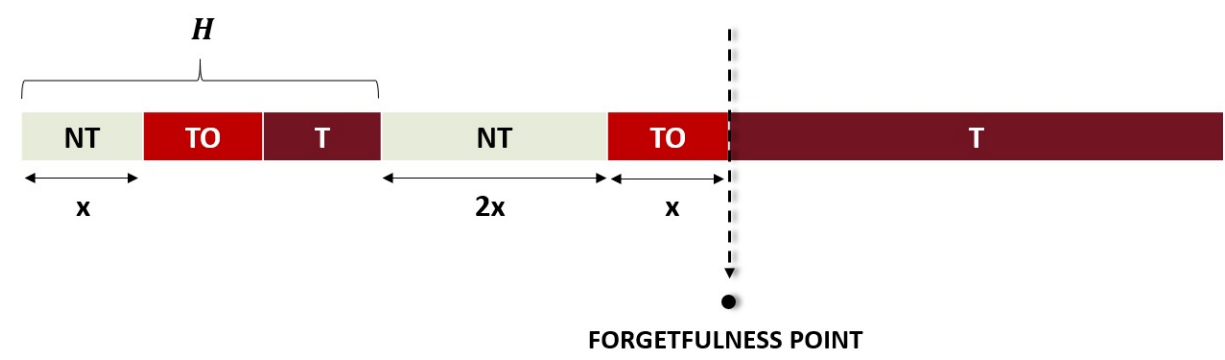

Figure 8.5: Forgetfulness Point. By fixing $H$ short enough we simulate the case in which at some point a cluster is forgotten by system. In the so-called forgetfulness point, the system forgets $T$ cluster

decided to maintain them separately to test the power of the synchronization measures and our system in distinguishing each individual state.

One might think that increasing the value of the horizon could fix the problem, however: 1) it would imply more memory use, a fact we want to avoid; and 2) using a large value of $H$, the cluster tails become longer, increasing the probability of overlapping clustering [167].

The solution could be to open the clusters and maintain them in memory, possibly as a background task. Remember that we would not maintain all the information and points of the clusters, just their CFs. To this end, I designed experiment 2, corresponding to Figure $8.4 \mathrm{~b}$, in which I wanted to simulate the behaviour of the system when opened clusters are not forgotten. As can be observed in the results for configuration 2 in table 8.2, the accuracy improves significantly, reaching $100 \%$ in all the observed cases. This is because when maintaining the learned clusters in the system, the confusion between $T O$ and $T$ does not occur. Note that keeping the information about the opened clusters is not equivalent to the classical approximation of training and testing. This is later addressed in the discussion section.

The obtained results in OP-2 show a very good level of immediacy and readiness since we were able to detect all changes of states (our concept drifts), adapting the output.

To control stochasticity levels in the outputs due to the online modification of the clusters, the determination of the rest of the system's hyperparameters has been done by fine-tuning. The percentage of change in radius increase, radius decrease and cluster addition has been set to $0.5,0.5$ and 0.3 respectively. Higher values lead to a loss of accuracy of the system. Finally, in our system, I do not allow the removal of created clusters or the joining of two clusters, since, as previously stated, the system achieves the best performance when the necessary clusters are opened and maintained in the system. In this respect, it has been verified that 


\begin{tabular}{|c|c|c|c|c|c|c|}
\hline \multirow{2}{*}{ FILE } & \multirow{2}{*}{ BAND } & \multirow{2}{*}{ FEATURE } & \multicolumn{2}{|c|}{ OP-1 } & \multicolumn{2}{|c|}{ OP-2 } \\
\hline & & & ACC & CMM & ACC & CMM \\
\hline \multirow{6}{*}{1} & \multirow{3}{*}{ TB } & PhTE & 66.89 & 0.6729 & 100 & 0.7614 \\
\hline & & MI & 66.89 & 0.6852 & 100 & 0.7837 \\
\hline & & BICOH & 66.89 & 0.66 & 100 & 0.7730 \\
\hline & \multirow{3}{*}{ LB } & PhTE & 73.87 & 0.7977 & 100 & 0.8318 \\
\hline & & MI & 73.87 & 0.8815 & 100 & 0.9298 \\
\hline & & BICOH & 73.87 & 0.852 & 100 & 0.9183 \\
\hline \multirow{6}{*}{2} & \multirow{3}{*}{ TB } & PhTE & 73.51 & 0.7284 & 100 & 0.7961 \\
\hline & & MI & 73.51 & 0.7089 & 100 & 0.7589 \\
\hline & & BICOH & 73.51 & 0.7049 & 100 & 0.7653 \\
\hline & \multirow{3}{*}{ LB } & PhTE & 51.73 & 0.8704 & 100 & 0.93 \\
\hline & & MI & 51.73 & 0.8595 & 100 & 0.8907 \\
\hline & & BICOH & 51.73 & 0.8447 & 100 & 0.8628 \\
\hline \multirow{6}{*}{3} & \multirow{3}{*}{ TB } & PhTE & 50.81 & 0.6063 & 100 & 0.9201 \\
\hline & & MI & 50.81 & 0.6250 & 100 & 0.9071 \\
\hline & & BICOH & 50.81 & 0.6144 & 100 & 0.9665 \\
\hline & \multirow{3}{*}{ LB } & PhTE & 51.73 & 0.6914 & 100 & 0.9880 \\
\hline & & MI & 51.73 & 0.6670 & 100 & 0.9835 \\
\hline & & BICOH & 51.73 & 0.6660 & 100 & 0.9812 \\
\hline \multirow{6}{*}{4} & \multirow{3}{*}{ TB } & PhTE & 84.47 & 0.8228 & 100 & 0.9144 \\
\hline & & MI & 84.47 & 0.8228 & 100 & 0.9483 \\
\hline & & BICOH & 84.47 & 0.8899 & 100 & 0.9264 \\
\hline & \multirow{3}{*}{ LB } & PhTE & 77.90 & 0.5972 & 100 & 0.9741 \\
\hline & & MI & 77.90 & 0.5641 & 100 & 0.9870 \\
\hline & & BICOH & 77.90 & 0.9768 & 100 & 0.9810 \\
\hline
\end{tabular}

Table 8.2: Results of Stream Clustering for configurations OP-1 in which clusters are not maintained in the system and OP-2 in which the system takes advantage of the peri-operative period initializing the clusters in the system

the rate of 0.3 in the cluster addition hyperparameter is sufficient to open only the necessary clusters. i.e, no more than the three necessary clusters are opened.

\section{Comparing DSM with a traditional clustering approach}

In this section I present results of accuracy for two classical and popular clustering approaches for the sake of comparing them with stream clustering. Both chosen because they carry relatively little computation: i) K-means++ [173] using 3 clusters and ii)a density-based clustering using a canopy algorithm [174], with 3 clusters; a periodic pruning rate of 0 to avoid deleting open clusters (as in stream clustering); The tight distance T2 and loss distance T1 have been set individually for each record: T2 has been set based on the feature (synchronization index) standard deviation as $S D=0.5 * S D /(\max -\min )$; and $T 1=T 2 * 1.25$.

I perform the analysis by considering the features both alone and combined, to improve the results of the classical clustering techniques. However, as can be seen in table 8.3, the results of classical techniques are far from the performance obtained with DSM.

The results confirm our initial hypothesis, which is why I opted for DSM: neural activity constitutes a source of non-linear and non-stationary data. For that reason, a system able to adapt itself, learning in a continuous way would benefit the performance, increasing the global accuracy significantly and using the IMD resources much more effectively. 


\begin{tabular}{|c|c|c|c|c|c|}
\hline File & Band & Feature Subset & $\begin{array}{l}\text { Stream- } \\
\text { Clustering }\end{array}$ & K-means++ & $\begin{array}{c}\text { Density-based } \\
\text { (Canopy) }\end{array}$ \\
\hline \multirow{8}{*}{1} & \multirow{4}{*}{ TB } & PhTE & 100 & 46.82 & 45.17 \\
\hline & & MI & 100 & 44.37 & 52.98 \\
\hline & & $\mathrm{BiCOH}$ & 100 & 30.46 & 34.43 \\
\hline & & $\begin{array}{c}\text { PhTE + MI + } \\
\text { BiCOH }\end{array}$ & - & 59.60 & 50.99 \\
\hline & \multirow{4}{*}{ LB } & PhTE & 100 & 58.62 & 51.54 \\
\hline & & MI & 100 & 57.89 & 58.07 \\
\hline & & $\mathrm{BiCOH}$ & 100 & 54.44 & 56.3 \\
\hline & & $\begin{array}{c}\text { PhTE + MI + } \\
\text { BiCOH }\end{array}$ & - & 48.27 & 60.25 \\
\hline \multirow{8}{*}{2} & \multirow{4}{*}{ TB } & PhTE & 100 & 45.69 & 46.35 \\
\hline & & MI & 100 & 54.96 & 53.64 \\
\hline & & $\mathrm{BiCOH}$ & 100 & 45.35 & 54.3 \\
\hline & & $\begin{array}{c}\text { PhTE + MI + } \\
\text { BiCOH }\end{array}$ & - & 50.34 & 55.63 \\
\hline & \multirow{4}{*}{ LB } & PhTE & 100 & 57.35 & 60.60 \\
\hline & & MI & 100 & 54.80 & 50.81 \\
\hline & & $\mathrm{BiCOH}$ & 100 & 59.53 & 62.79 \\
\hline & & $\begin{array}{c}\text { PhTE + MI + } \\
\text { BiCOH }\end{array}$ & - & 43.38 & 55.35 \\
\hline \multirow{8}{*}{3} & \multirow{4}{*}{ TB } & PhTE & 100 & 61.3 & 59.67 \\
\hline & & MI & 100 & 47.73 & 39.61 \\
\hline & & $\mathrm{BiCOH}$ & 100 & 55.78 & 54.15 \\
\hline & & PhTE + MI + BICOH & - & 48.92 & 48.64 \\
\hline & \multirow{4}{*}{ LB } & PhTE & 100 & 63.43 & 53.83 \\
\hline & & MI & 100 & 62.54 & 45.06 \\
\hline & & $\mathrm{BiCOH}$ & 100 & 63.59 & 55.24 \\
\hline & & $\begin{array}{c}\text { PhTE + MI + } \\
\text { BiCOH }\end{array}$ & - & 36.85 & 52.5 \\
\hline \multirow{8}{*}{4} & \multirow{4}{*}{ TB } & PhTE & 100 & 52.01 & 50.31 \\
\hline & & MI & 100 & 54.03 & 51.55 \\
\hline & & $\mathrm{BiCOH}$ & 100 & 52.01 & 51.08 \\
\hline & & $\begin{array}{c}\mathrm{PhTE}+\mathrm{MI}+ \\
\mathrm{BiCOH}\end{array}$ & - & 48.76 & 43.78 \\
\hline & \multirow{4}{*}{ LB } & PhTE & 100 & 64.39 & 61.14 \\
\hline & & MI & 100 & 58.82 & 53.89 \\
\hline & & $\mathrm{BiCOH}$ & 100 & 61.46 & 55.8 \\
\hline & & $\begin{array}{c}\text { PhTE + MI + } \\
\text { BiCOH }\end{array}$ & - & 37.84 & 57.38 \\
\hline
\end{tabular}

Table 8.3: Accuracy results comparison between stream and classical clustering 


\subsection{Discussion}

\section{Heterogeneity of LFP Connectivity across patients}

Previous studies on LFP-STN show that it presents a high degree of heterogeneity across patients $[69,158,175]$. This suggests that several connectivity patterns could exist, which have yet to be identified [132].

I have not delved deeply into this question, but the applied feature selection procedure reveals some information about it. Some synchronisation measures are more stable across patients, while others are more specific, not showing changes in all cases, as is the case of PLV and PhTE.

Despite the inter-subject variability, the connectivity patterns revealed from some indexes perfectly detect the change between tremor states in all the cases. This is precisely the reason why they have been selected as features, while others cannot accurately distinguish among states, and was discarded. Certainly, more studies in this direction are necessary.

\section{Preferred Frequency Bands}

As mentioned before, previous studies have found that local synchronization in the beta band is linked with bradykinesia and rigidity, but not with the tremor. Our results show that the connectivity fluctuations when tremor appears are more appreciable on the tremor and the lower beta bands. I have observed, as in other studies that not all the beta band is involved at the same level in PD symptomatology $[162,163]$.

\section{Application of LFP connectivity to closed-loop DBS}

Presently, PD has no cure. Therefore, the treatments are aimed at combating the associated symptomatology. The first option is, in most cases, is treatment based on levodopa. However, it can lead to numerous complications, and with the advance of the disease, some patients have to undergo surgery to change from pharmacological treatment to neurostimulation via an implantable medical device called a neurostimulator [176].

As commented in the introduction, both ECG signals and pathological events in cardiac diseases are well known; the first pacemaker dates back to 1958, while the first HFS-STN device (high-frequency stimulation of the STN) did not appear until 1993 [177, 178]. Moreover, we know less about neural oscillations because the signal is less accessible and more complex than ECG. Maybe for these reasons, neurostimulators and pacemakers do not work in the same way. More research work is needed concerning LFP connectivity and its relation to DBS [94]. In this sense, the main contribution of this paper is to find that some connectivity measures can distinguish with high accuracy between tremorous and atremorous states directly from LFP-STN activity, employing a stream clustering system. This approximation is appropriate for the closed-loop DBS problem since: 


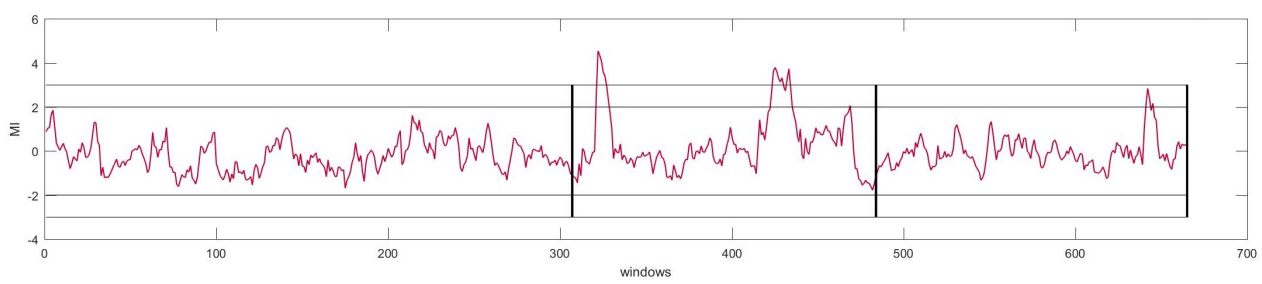

Figure 8.6: Dynamic Analysis of LFP-STN measured with MI over the tremor band. The left and right black vertical line represents the transition from $N T$ to $T O$ and from $T O$ to $T$, respectively. The horizontal lines represent the \pm 2 and \pm 3 standard deviation thresholds for statistical significance

1. It does not need any other measure. It only requires as input the LFP signal that can be recorded by the IPG.

2. The system has been tested in immediacy and readiness parameters, showing that it is able to detect the change between states in real-time, with no delay and with $100 \%$ accuracy.

Of course, as mentioned previously, these good results are not only the outcome of the stream clustering system but of the connectivity indexes employed as meters of LFP activity. In fact, if we perform analysis into the dynamic of such measurements, we can see how they reflect the drifts between states and that the mean level of synchronisation varies across states. Figure 8.6 shows an example of dynamics measured by MI index calculated over the tremor band.

As a last observation, as depicted in Figure 8.6, the physical symptoms appear in the $T$ period. Thus, since we are able to separate TO instances from $T$ instances, we could decide whether to stimulate only in the $T$ period or from the beginning of TO. We could even prepare the IPG once the TO instances arrive at the clustering, and launch the stimulation when the $T$ instances appear. All the possibilities will be perfectly possible with rigorous accuracy in the presented system.

\section{Maintain Opened Clusters Strategy}

As stated in section 8.7, the system is conceived to be initialised during the perioperative period. To this end, the system observes the activity of the STN during the necessary time to watch the patient transiting between the movement states, and thus it is able to open the required clusters

I have tested several experiments to observe the effect that forgetting the initialised clusters has in classifying the subsequent instances, and subsequently in the accuracy of the system. The best results were though obtained when we maintained the opened clusters in the system since the possibility of confusing an arriving instance to a similar one belonging to another cluster reduced drastically. Nonetheless, due to the conclusions obtained from experimentation, I strongly recommend to initialise and maintain the clusters opened in the system, to avoid misclassification. 


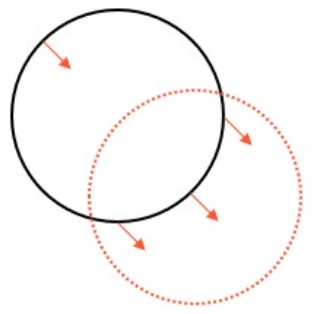

a)

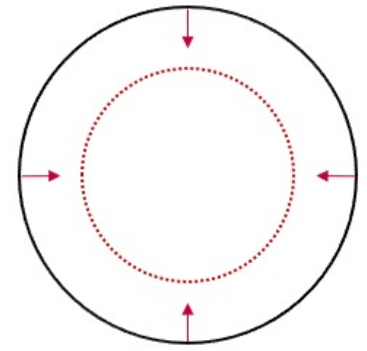

b)

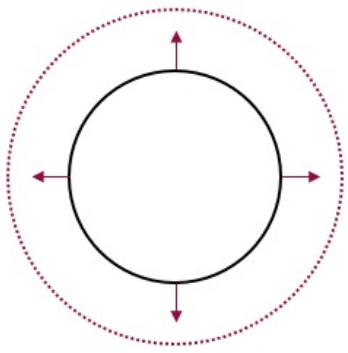

c)

Figure 8.7: Ways of updating clusters in stream clustering technique. a) cluster re-positioned. b) radius decrease and c) radius increase. The direction of the arrows indicates in which direction the cluster evolves

As previously stated, this approximation is not equivalent to the classical approximation of training and testing for two main reasons:

1. In classical clustering, the centroids of the clusters are set during the training phase and remain constant afterwards. When we need to know the class of an instance, we evaluate to which cluster it belongs.

In stream clustering, we update the cluster structure after the inclusion of the new sample: The centroids of clusters can be re-positioned, and the radius of the cluster can increase or decrease, as illustrated in Figure 8.7.

2. In classical clustering, all the information about the clusters is maintained in memory, whilst DSM algorithms operate using a limited amount of memory. To this end the stream clustering algorithm stores the so-called CFs, presented in section five, and the structure to maintain only that strictly necessary to operate. This information is updated each step, evolving at the time and form STN-LFP activity does.

\section{Stochasticity of the System}

There are two components in the system that can be stochastic in nature:

1. The inputs. We could find two sources of stochasticity in the inputs:

(a) The noise present in the data. To reduce the effects of possible noise in the data, recordings have been pre-processed as presented in section 8.2 .

(b) The peak frequency of Parkinsonian tremor may not always be at the same frequency varying slightly, not only across subjects but also in different windows of observation of the same subject. In order to be sure we capture the tremor peak, two approaches can be taken: 
i) Identify the peak frequency between the 3-7 Hz band, denoted with $f p$, and filter the signal between $(f p-0.5)$ and $(f p+0.5)$. Note here that the peak frequency in PD has an approximate bandwidth of $1 \mathrm{~Hz}$. However, the tremor peak is not always exactly at the same frequency so that, to be sure to capture the peak, we should perform an ad-hoc detection and filtering process for each signal window. This approach would be very expensive, given the restricted capabilities of energy, and the computing power of the IMDs, which would limit the application of our system.

ii) Use the entire tremor [3-7] band. In this respect, the results show that the selection of the whole tremor band gives high accuracy, probably because, being the tremor a narrow peak, most of the power in the band is concentrated surrounding this peak, acting as a natural narrow-pass filter. I checked the data and, in fact, up to $78.7 \%$ of the power of the band falls within $1 \mathrm{~Hz}$ of the tremor peak. A recent paper [42] showed that, when using Hilbert transform (as in PhTE), the existence of a clear frequency peak is enough to ensure that the phase is extracted correctly, and it is not needed to use a narrower band. This is probably also true for $\mathrm{MI}$ and $\mathrm{BiCOH}$.

2. The cluster movements: To control stochasticity levels in the outputs due to an incorrect update of the clusters, the selection of the system's hyperparameters that control such updates has been determined by fine tunning, as stated in section 8.7. 



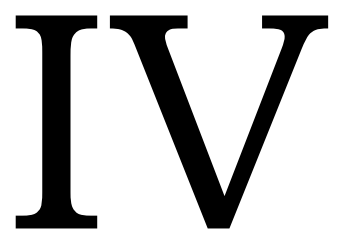

\section{Conclusions}

We can only see a short distance ahead, but we can see plenty there that needs to be done.

Alan Turing

Imagination is the discovering faculty, pre-eminently. It is that which penetrates the unseen worlds around us, the worlds of Science. Ada Lovelace 



\section{9}

\section{General Conclusions \& Future Perspectives}

Conclusions have been drawn in each of the chapters (corresponding to the different studies carried out) of this thesis. Nevertheless, here, a few lines are devoted to drawing general conclusions about the future of DBS and medicine in general.

\section{General Conclusions}

eep brain stimulation has proven to be an effective solution for the treatment of movement disorders, especially in cases where oral treatment is not enough. However, the exact way this stimulation operates in the brain remains a mystery. Furthermore, current devices operate in an open-loop manner, not being possible to adjust stimulation parameters based on the clinical state of the patient at all times. Although it is, in general, a good treatment, which improves the quality of life of patients, continuous stimulation produces some adverse effects. Clinical experience has associated these adverse effects with continuous stimulation. On the other hand, continuous stimulation makes inefficient use of the device's battery.

These drawbacks of the DBS can be mitigated using new closed-loop strategies. In the case of demand-driven DBS strategies, the objective is to adapt the functioning of the device in real-time in response to changes in clinical (motor) status experienced by the patient. Several studies, including significant device development companies, agree that closed-loop strategies will be the therapy implemented in future DBS systems. To this end, the new generation of DBS devices must be able to sense the electrical signal in the target area while simultaneously delivering therapy. These devices will be able to obtain artefact-free LFP recordings during stimulation. For this purpose, from the industry side, Medtronic developed the Activa ${ }^{\circledR} P C+S$ neurostimulator, which is only available for research so far, but that points the way to future neurostimulation systems [179]. 
Some works in the area of bioengineering and neuroscience are starting to propose the use of closed-loop systems to improve current treatments. These systems would have several advantages. On one side, these intelligent systems would save time and health resources in the programming of the device. But most importantly, these devices are expected to be able to mitigate the adverse effects that current DBS induces.

Five studies (among which there are four published papers) have been presented in this thesis, investigating the effects exerted by DBS and proposing closed-loop DBS systems. In the last one, $100 \%$ accuracy is achieved. This suggests that this type of solutions are possible and could suppose a more effective and personalised treatment of Parkinson's disease.

\section{Future Perspectives}

\section{Future of DBS}

All the proposed closed-loop systems are focused on the current neurostimulation systems, devices that, as we have seen, are implantable. However, a fascinating work proposed recently by Dr. Nir Grossman raises the development of non-invasive DBS systems [180]. The key idea is to stimulate the target region, which is a deep structure of the brain, using external electrodes placed on the surface of the head and avoiding stimulating the rest of the brain areas. They resolve this through the concept they call Temporal Interference, which consists of stimulating externally at different points at different frequencies. The fields created interfere between them, cancelling or strengthening, in the brain areas of interest.

The fact that the current devices are implantable supposes a problem in the development of all the improvements, that in my point of view, the deep brain stimulation needs. Therefore, the work of Dr. Grossman opens the door so that they can be carried out. Apart from the fact that it is non-invasive as the authors propose, there are additional improvements that in my opinion should be implemented in future DBS systems, which include:

An integrated closed-loop solution . As previously presented in chapter four, closed-loop neurostimulation is an umbrella term which encompasses different DBS strategies. Some of them focus on real-time adjustment of the stimulation parameters. Others, such as those proposed in this thesis, aim to determine when stimulation is needed. Future DBS systems must bring both strategies together, being able to provide the stimulation at the right time and to the right extent.

A precise stimulation approach . The influence of the DBS is not limited to the target area, where the electrodes are inserted. As seen in chapter three, it also has influence at the cortical level. In this chapter, it has been hypothesised that some of the cortical effects are adverse, by modifying areas that should not be 
modified.

Intervening in the cortical areas that are modified with current devices is difficult, or rather impossible (since we cannot control how the signal propagates inside the brain by stimulating at a single point). However, if non-invasive devices are used as proposed by Dr. Grossman, stimulation algorithms could be designed to influence the desired way in each of the areas, avoiding the adverse effects described in Chapter three. Moreover, applying the method of analysis described in chapter three, comparing the OFF and ON status of a patient, can determine the effects of stimulation on that patient. Thus, the treatment could even be individualized.

Cybersecurity measures ! . Computer security measures are always the great forgotten ones. As an example, when ARPANET (the first computer network and the precursor of the Internet) was put into operation, communication protocols were developed that connected computers that shared information with each other, forming a connected network. None of these protocols took into account the fact that this network could present vulnerabilities to its security. It was not until 1973 when ARPANET had already been intruded that the first network security considerations were born. Today it would be unthinkable not to implement security measures in an environment such as the Internet. However, throughout history, there have been many examples like this, in which security has always been applied a posteriori, once the problems have already arisen.

The new generation of medical devices are hackable [149]. Thus, an adversary with computer skills can compromise patient safety by passive attacks, such as spoofing, or active attacks, as the change of the device configuration parameters. In the case of neurostimulators, for example, an active attacker could cause impairment of motor function, alteration of impulse control, modification of emotions or affect induction of pain, and modulation of the reward system [181].

Therefore, in my opinion, is vital new systems take into account security measures from the design phase of the device, thus covering its vulnerabilities and avoiding attacks as much as possible. Note that this takes on particular relevance not only in medical devices but in all coming neural devices, that connects brain and computers. Therefore, I intuit that cybersecurity in $\mathrm{BCI}$ is going to become increasingly important in the scientific community in the next few years.

\section{Future of Medicine and beyond}

In the not-so-distant future, medicine will be led by engineering. Intelligent systems will give quicker and more precise answers, with an infinitely smaller number of failures, to our diseases. In fact, these systems are not so far away, good examples are the IBM Watson expert system (figure 9.1), which is capable of inferring diagnoses through the use of a big data architecture that contains medical data of all kinds. It is merely as if a person had as much information as possible about a case: what happened in other cases, what complications may arise, everything. In 
a few years, these systems will outpace today's doctors.

Another example is the Da Vinci robot (figure 9.2), a robotic system developed in Silicon Valley. It is the latest and most recent evolution of minimally invasive surgery. The use of the Da Vinci robot ensures greater firmness in the surgical technique, providing precision, minimum invasiveness and safety in surgical interventions.

In the next years, neuroscience will cross obstacles that have not been crossed in the last century. It is expected that in the near future, there will be new and increasingly powerful devices aimed at creating brain-computer interfaces to connect humans and machines. In this sense, new neuronal devices of limited dimensions are being developed. These systems suppose a brain-computer interface with a temporal and spatial resolution higher than the current ones. An example of these devices is the Neural dust (shown in figure 9.3).

There are already companies working in advanced BCI systems. One of them is Neuralink, the company created by Elon Musk claims it will have the technology to fuse human brains with machines in a decade. Another example is Kernel, which is developing chips to be inserted into the human brain, capable of being implanted laparoscopically and using other less invasive methods. The main objective of their chips is the treatment of neurological diseases such as stroke and Alzheimer's disease. But the final objective of this company is to develop implants that improve the cognition of any user, not only ones affected by dementia.

Definitely a new and exciting era will come with all these developments. 


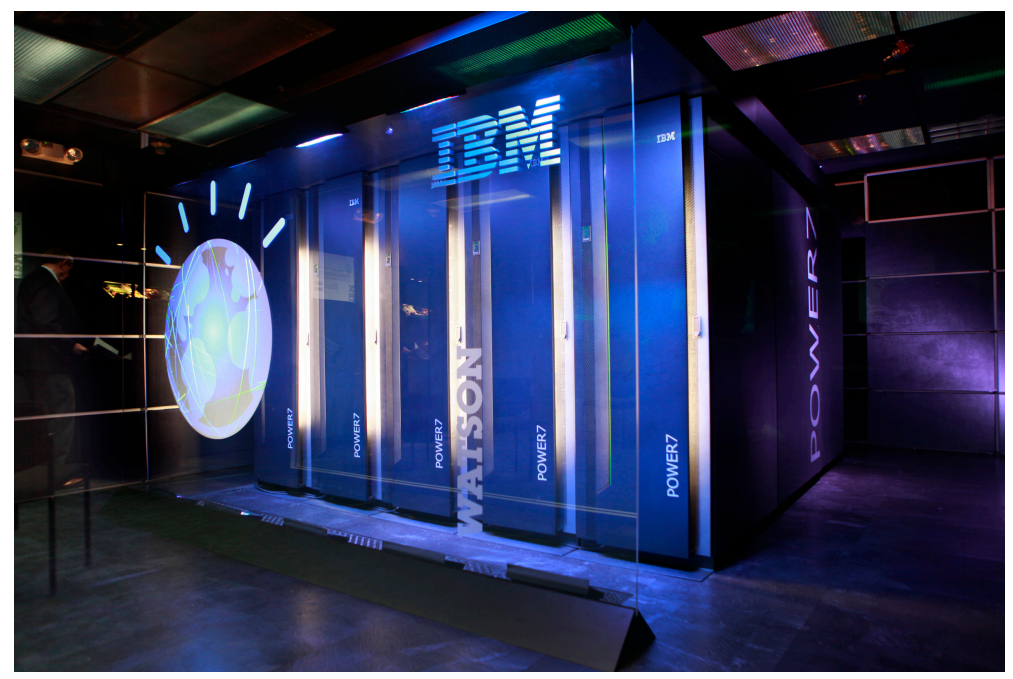

Figure 9.1: IBM Watson. Watson is a computerized answer search system developed by IBM, which describes it as "an application of advanced technologies designed for natural language processing, information retrieval, knowledge representation, automatic reasoning, and automatic learning to the open field of answer searches".
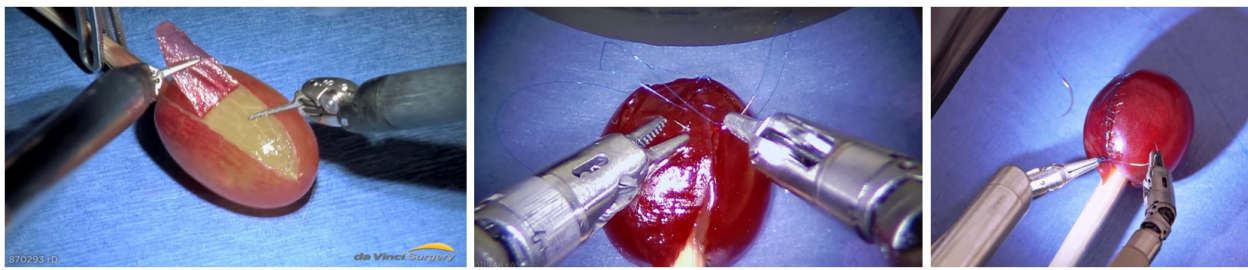

Figure 9.2: Da Vinci surgical robot peeling a grape and sewing it afterwards

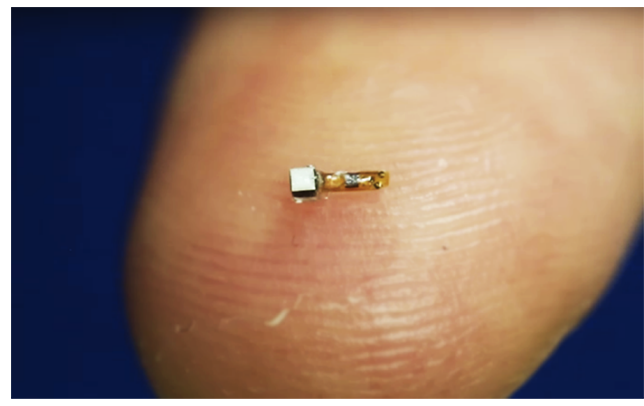

Figure 9.3: Neural Dust neural sensors 



\section{Curriculum Vitæ}

\section{Education}

2018

Research Visit

Oxford University, Oxford, UK.

2018

$\mathrm{PhD}$. Computer Science

Carlos III University of Madrid

Thesis title: Cybersecurity in implantable medical devices

Best Doctoral Thesis in Cybersecurity at RENIC 2018 National Awards.

2016

Research Visit

Aalto University, Helsinki, Finland.

$2014 \quad$ MsC. Biomedical Engineering

(Signal Processing and Devices Specialization Track)

Technical University of Madrid

2011

MsC. Computer Science and Technology

(Artificial Intelligence Specialization Track)

Carlos III University of Madrid

2010 Degree in Computer Engineering

(Software Engineering Specialization Track)

Carlos III University of Madrid 



\section{Bibliography}

[1] Joseph Jankovic and Eduardo Tolosa. Parkinson's disease and movement disorders. Lippincott Williams \& Wilkins, 2007.

[2] Wolf Singer. Understanding the brain. EMBO reports, 8(S1):S16-S19, 2007.

[3] J Parkinson. An essay on the shaking palsy: London: Whittingham and rowland for sherwood. Neely and Jones, 1817.

[4] YF Tai and P Piccini. Applications of positron emission tomography (pet) in neurology. Journal of Neurology, Neurosurgery \& Psychiatry, 75(5):669-676, 2004.

[5] Matthew James Farrer. Genetics of parkinson disease: paradigm shifts and future prospects. Nature Reviews Genetics, 7(4):306, 2006.

[6] Tamara Pringsheim, Nathalie Jette, Alexandra Frolkis, and Thomas DL Steeves. The prevalence of parkinson's disease: A systematic review and meta-analysis. Movement disorders, 29(13):1583-1590, 2014.

[7] Robert L Nussbaum and Christopher E Ellis. Alzheimer's disease and parkinson's disease. New england journal of medicine, 348(14):1356-1364, 2003.

[8] E Ray Dorsey and Bastiaan R Bloem. The parkinson pandemic-a call to action. JAMA neurology, 75(1):9-10, 2018.

[9] Ilana Schlesinger, Ayelet Eran, Alon Sinai, Ilana Erikh, Maria Nassar, Dorith Goldsher, and Menashe Zaaroor. Mri guided focused ultrasound thalamotomy for moderate-to-severe tremor in parkinson's disease. Parkinson's Disease, 2015, 2015.

[10] Yilong Ma, Shichun Peng, Vijay Dhawan, and David Eidelberg. Dopamine cell transplantation in parkinson's disease: challenge and perspective. British medical bulletin, 100(1):173-189, 2011.

[11] John Gardner. A history of deep brain stimulation: technological innovation and the role of clinical assessment tools. Social studies of science, 43(5):707728, 2013.

[12] L Yap, A Kouyialis, and TRK Varma. Stereotactic neurosurgery for disabling tremor in multiple sclerosis: thalamotomy or deep brain stimulation? British journal of neurosurgery, 21(4):349-354, 2007. 
[13] Tobias Loddenkemper, Andrew Pan, Silvia Neme, Kenneth B Baker, Ali R Rezai, Dudley S Dinner, Erwin B Montgomery Jr, and Hans O Lüders. Deep brain stimulation in epilepsy. Journal of Clinical Neurophysiology, 18(6):514$532,2001$.

[14] Helen S Mayberg, Andres M Lozano, Valerie Voon, Heather E McNeely, David Seminowicz, Clement Hamani, Jason M Schwalb, and Sidney H Kennedy. Deep brain stimulation for treatment-resistant depression. Neuron, 45(5):651-660, 2005.

[15] Erlick AC Pereira, Alexander L Green, Richard J Stacey, and Tipu Z Aziz. Refractory epilepsy and deep brain stimulation. Journal of clinical neuroscience, 19(1):27-33, 2012.

[16] Adrian W Laxton and Andres M Lozano. Deep brain stimulation for the treatment of alzheimer disease and dementias. World neurosurgery, 80(3-4):S28e1, 2013.

[17] Sarah LF Owen, Alexander L Green, John F Stein, and Tipu Z Aziz. Deep brain stimulation for the alleviation of post-stroke neuropathic pain. Pain, 120(1-2):202-206, 2006.

[18] Jean Siegfried and Bodo Lippitz. Bilateral chronic electrostimulation of ventroposterolateral pallidum: a new therapeutic approach for alleviating all parkinsonian symptoms. Neurosurgery, 35(6):1126-1130, 1994.

[19] R Pahwa, SA Factor, KE Lyons, WG Ondo, G Gronseth, H Bronte-Stewart, M Hallett, J Miyasaki, J Stevens, and WJ Weiner. Practice parameter: Treatment of parkinson disease with motor fluctuations and dyskinesia (an evidence-based review):[retired]: Report of the quality standards subcommittee of the american academy of neurology. Neurology, 66(7):983-995, 2006.

[20] Alim L Benabid, Abdelhamid Benazzous, and Pierre Pollak. Mechanisms of deep brain stimulation. Movement disorders: official journal of the Movement Disorder Society, 17(S3):S73-S74, 2002.

[21] JA Obeso, CW Olanow, MC Rodriguez-Oroz, P Krack, R Kumar, and AE Lang. Deep brain stimulation for parkinson's disease study group. deep-brain stimulation of the subthalamic nucleus or the pars interna of the globus pallidus in parkinson's disease. N Engl J Med, 345(13):956-963, 2001.

[22] Jens Volkmann, Niels Allert, Jürgen Voges, Volker Sturm, Alfons Schnitzler, and Hans-Joachim Freund. Long-term results of bilateral pallidal stimulation in parkinson's disease. Annals of Neurology: Official Journal of the American Neurological Association and the Child Neurology Society, 55(6):871-875, 2004. 
[23] J Volkmann, N Allert, J Voges, PH Weiss, H-J Freund, and V Sturm. Safety and efficacy of pallidal or subthalamic nucleus stimulation in advanced pd. Neurology, 56(4):548-551, 2001.

[24] Jeff M Bronstein, Michele Tagliati, Ron L Alterman, Andres M Lozano, Jens Volkmann, Alessandro Stefani, Fay B Horak, Michael S Okun, Kelly D Foote, Paul Krack, et al. Deep brain stimulation for parkinson disease: an expert consensus and review of key issues. Archives of neurology, 68(2):165-165, 2011.

[25] SJ Groiss, L Wojtecki, M Südmeyer, and A Schnitzler. Deep brain stimulation in parkinson's disease. Therapeutic advances in neurological disorders, 2(6):379-391, 2009.

[26] Ali Sarem-Aslani and Keith Mullett. Industrial perspective on deep brain stimulation: history, current state, and future developments. Frontiers in integrative neuroscience, 5:46, 2011.

[27] A Amon and F Alesch. Systems for deep brain stimulation: review of technical features. Journal of Neural Transmission, 124(9):1083-1091, 2017.

[28] Gang Li, Hao Su, Gregory A Cole, Weijian Shang, Kevin Harrington, Alex Camilo, Julie G Pilitsis, and Gregory S Fischer. Robotic system for mri-guided stereotactic neurosurgery. IEEE Transactions on Biomedical Engineering, 62(4):1077-1088, 2014.

[29] Gennady G Knyazev. Motivation, emotion, and their inhibitory control mirrored in brain oscillations. Neuroscience \& Biobehavioral Reviews, 31(3):377395, 2007.

[30] Brian H Bland and Scott D Oddie. Theta band oscillation and synchrony in the hippocampal formation and associated structures: the case for its role in sensorimotor integration. Behavioural brain research, 127(1-2):119-136, 2001.

[31] Chang S Nam, Yongwoong Jeon, Young-Joo Kim, Insuk Lee, and Kyungkyu Park. Movement imagery-related lateralization of event-related (de) synchronization (erd/ers): motor-imagery duration effects. Clinical Neurophysiology, 122(3):567-577, 2011.

[32] Dany Bright, Amrita Nair, Devashish Salvekar, and Swati Bhisikar. Eeg-based brain controlled prosthetic arm. In 2016 Conference on Advances in Signal Processing (CASP), pages 479-483. IEEE, 2016.

[33] Christa Neuper, Reinhold Scherer, Selina Wriessnegger, and Gert Pfurtscheller. Motor imagery and action observation: modulation of sensorimotor brain rhythms during mental control of a brain-computer interface. Clinical neurophysiology, 120(2):239-247, 2009. 
[34] B. J. Harrison and C. Pantelis. Local field Potencials. Encyclopedia of Psychopharmacology. Springer Berlin Heidelberg, 2010.

[35] Q. Gaucher, J. M. Edeline, and B. Gourevitch. How different are the local field potentials and spiking activities insight from multi-electrodes arrays. Journal of Physiology-Paris, 106(3):93-103, 2012.

[36] György Buzsáki, Costas A Anastassiou, and Christof Koch. The origin of extracellular fields and currents-eeg, ecog, Ifp and spikes. Nature reviews neuroscience, 13(6):407, 2012.

[37] Matthew J Nelson and Pierre Pouget. Do electrode properties create a problem in interpreting local field potential recordings?, 2010.

[38] David A Markowitz, Yan T Wong, Charles M Gray, and Bijan Pesaran. Optimizing the decoding of movement goals from local field potentials in macaque cortex. Journal of Neuroscience, 31(50):18412-18422, 2011.

[39] CJ Stam. Use of magnetoencephalography (meg) to study functional brain networks in neurodegenerative disorders. Journal of the neurological sciences, 289(1-2):128-134, 2010.

[40] Samu Taulu and Juha Simola. Spatiotemporal signal space separation method for rejecting nearby interference in meg measurements. Physics in Medicine \& Biology, 51(7):1759, 2006.

[41] Robert Oostenveld, Pascal Fries, Eric Maris, and Jan-Mathijs Schoffelen. Fieldtrip: open source software for advanced analysis of meg, eeg, and invasive electrophysiological data. Computational intelligence and neuroscience, 2011:1, 2011.

[42] Ricardo Bruña, Fernando Maestú, and Ernesto Pereda. Phase locking value revisited: teaching new tricks to an old dog. Journal of neural engineering, 15(5):056011, 2018.

[43] Fernando Maestú, Ernesto Pereda, and Francisco Del Pozo. Conectividad funcional y anatómica en el cerebro humano, 2015.

[44] Guido Nolte, Ou Bai, Lewis Wheaton, Zoltan Mari, Sherry Vorbach, and Mark Hallett. Identifying true brain interaction from eeg data using the imaginary part of coherency. Clinical neurophysiology, 115(10):2292-2307, 2004.

[45] Ernesto Pereda, Rodrigo Quian Quiroga, and Joydeep Bhattacharya. Nonlinear multivariate analysis of neurophysiological signals. Progress in neurobiology, 77(1-2):1-37, 2005.

[46] Andreas Bruns, Reinhard Eckhorn, Hennric Jokeit, and Alois Ebner. Amplitude envelope correlation detects coupling among incoherent brain signals. Neuroreport, 11(7):1509-1514, 2000. 
[47] Andreas K Engel, Christian Gerloff, Claus C Hilgetag, and Guido Nolte. Intrinsic coupling modes: multiscale interactions in ongoing brain activity. Neuron, 80(4):867-886, 2013.

[48] Michael G Rosenblum, Arkady S Pikovsky, and Jürgen Kurths. Phase synchronization of chaotic oscillators. Physical review letters, 76(11):1804, 1996.

[49] Jean-Philippe Lachaux, Eugenio Rodriguez, Jacques Martinerie, Francisco ] Varela, et al. Measuring phase synchrony in brain signals. Human brain mapping, 8(4):194-208, 1999.

[50] Claude E. Shannon and Warren Weaver. The Mathematical Theory of Information. University of Illinois Press, 1949.

[51] Muriel Lobier, Felix Siebenhühner, Satu Palva, and J Matias Palva. Phase transfer entropy: a novel phase-based measure for directed connectivity in networks coupled by oscillatory interactions. Neuroimage, $85: 853-872$, 2014.

[52] D Wong, DA Clifton, and L Tarassenko. An introduction to the bispectrum for eeg analysis. In Postgraduate Conference in Biomedical Engineering \& Medical Physics, pages 61-62, 2009.

[53] Jerry M Mendel. Tutorial on higher-order statistics (spectra) in signal processing and system theory: Theoretical results and some applications. Proceedings of the IEEE, 79(3):278-305, 1991.

[54] Cornelis J Stam. Nonlinear dynamical analysis of eeg and meg: review of an emerging field. Clinical neurophysiology, 116(10):2266-2301, 2005.

[55] Narayan Puthanmadam Subramaniyam. Recurrence network analysis of eeg signals: A geometric approach. 2016.

[56] Kevin Warwick. Artificial intelligence: the basics. Routledge, 2013.

[57] Eric Maris and Robert Oostenveld. Nonparametric statistical testing of eegand meg-data. Journal of neuroscience methods, 164(1):177-190, 2007.

[58] A Benazzouz, DM Gao, ZG Ni, B Piallat, R Bouali-Benazzouz, and AL Benabid. Effect of high-frequency stimulation of the subthalamic nucleus on the neuronal activities of the substantia nigra pars reticulata and ventrolateral nucleus of the thalamus in the rat. Neuroscience, 99(2):289-295, 2000.

[59] JO Dostrovsky, R Levy, JP Wu, WD Hutchison, RR Tasker, and AM Lozano. Microstimulation-induced inhibition of neuronal firing in human globus pallidus. Journal of neurophysiology, 84(1):570-574, 2000.

[60] Cameron C McIntyre, Marc Savasta, Lydia Kerkerian-Le Goff, and Jerrold L Vitek. Uncovering the mechanism (s) of action of deep brain stimulation: activation, inhibition, or both. Clinical neurophysiology, 115(6):1239-1248, 2004. 
[61] Constance Hammond, Hagai Bergman, and Peter Brown. Pathological synchronization in parkinson's disease: networks, models and treatments. Trends in neurosciences, 30(7):357-364, 2007.

[62] David Williams, Marina Tijssen, Gerard Van Bruggen, Andries Bosch, Angelo Insola, Vincenzo Di Lazzaro, Paolo Mazzone, Antonio Oliviero, Angelo Quartarone, Hans Speelman, et al. Dopamine-dependent changes in the functional connectivity between basal ganglia and cerebral cortex in humans. Brain, 125(7):1558-1569, 2002.

[63] B Pollok, V Krause, W Martsch, C Wach, A Schnitzler, and M Südmeyer. Motorcortical oscillations in early stages of parkinson's disease. The Journal of physiology, 590(13):3203-3212, 2012.

[64] S Little, A Pogosyan, AA Kuhn, and P Brown. Beta band stability over time correlates with parkinsonian rigidity and bradykinesia. Experimental neurology, 236(2):383-388, 2012.

[65] Jan Hirschmann, Tolga E Özkurt, Markus Butz, M Homburger, Saskia Elben, CJ Hartmann, Jan Vesper, Lars Wojtecki, and Alfons Schnitzler. Distinct oscillatory stn-cortical loops revealed by simultaneous meg and local field potential recordings in patients with parkinson's disease. Neuroimage, 55(3):1159-1168, 2011.

[66] Vladimir Litvak, Ashwani Jha, Alexandre Eusebio, Robert Oostenveld, Tom Foltynie, Patricia Limousin, Ludvic Zrinzo, Marwan I Hariz, Karl Friston, and Peter Brown. Resting oscillatory cortico-subthalamic connectivity in patients with parkinson's disease. Brain, 134(2):359-374, 2010.

[67] Hagai Bergman and Günther Deuschl. Pathophysiology of parkinson's disease: from clinical neurology to basic neuroscience and back. Movement disorders: official journal of the Movement Disorder Society, 17(S3):S28S40, 2002.

[68] Elodie Lalo, Stéphane Thobois, Andrew Sharott, Gustavo Polo, Patrick Mertens, Alek Pogosyan, and Peter Brown. Patterns of bidirectional communication between cortex and basal ganglia during movement in patients with parkinson disease. Journal of Neuroscience, 28(12):3008-3016, 2008.

[69] Diane Whitmer, Camille De Solages, Bruce C Hill, Hong Yu, Jaimie M Henderson, and Helen Bronte-Stewart. High frequency deep brain stimulation attenuates subthalamic and cortical rhythms in parkinson's disease. Frontiers in human neuroscience, 6:155, 2012.

[70] Ashwini Oswal, Martijn Beudel, Ludvic Zrinzo, Patricia Limousin, Marwan Hariz, Tom Foltynie, Vladimir Litvak, and Peter Brown. Deep brain stimulation modulates synchrony within spatially and spectrally distinct resting state networks in parkinson's disease. Brain, 139(5):1482-1496, 2016. 
[71] Chunyan Cao, Dianyou Li, Tianxiao Jiang, Nuri Firat Ince, Shikun Zhan, Jing Zhang, Zhiyi Sha, and Bomin Sun. Resting state cortical oscillations of patients with parkinson disease and with and without subthalamic deep brain stimulation: a magnetoencephalography study. Journal of Clinical Neurophysiology, 32(2):109-118, 2015.

[72] JP Mäkelä, S Taulu, J Pohjola, A Ahonen, and E Pekkonen. Effects of subthalamic nucleus stimulation on spontaneous sensorimotor meg activity in a parkinsonian patient. In International Congress Series, volume 1300, pages 345-348. Elsevier, 2007.

[73] Omid Abbasi, Jan Hirschmann, Lena Storzer, Tolga Esat Özkurt, Saskia Elben, Jan Vesper, Lars Wojtecki, Georg Schmitz, Alfons Schnitzler, and Markus Butz. Unilateral deep brain stimulation suppresses alpha and beta oscillations in sensorimotor cortices. NeuroImage, 174:201-207, 2018.

[74] Katja Airaksinen, Anna Butorina, Eero Pekkonen, Jussi Nurminen, Samu Taulu, Antti Ahonen, Alfons Schnitzler, and Jyrki P Mäkelä. Somatomotor mu rhythm amplitude correlates with rigidity during deep brain stimulation in parkinsonian patients. Clinical Neurophysiology, 123(10):2010-2017, 2012.

[75] Chun-Yan Cao, Ke Zeng, Dian-You Li, Shi-Kun Zhan, Xiao-Li Li, and Bo-Min Sun. Modulations on cortical oscillations by subthalamic deep brain stimulation in patients with parkinson disease: A meg study. Neuroscience letters, 636:95-100, 2017.

[76] Jarkko Luoma, Eero Pekkonen, Katja Airaksinen, Liisa Helle, Jussi Nurminen, Samu Taulu, and Jyrki P Mäkelä. Spontaneous sensorimotor cortical activity is suppressed by deep brain stimulation in patients with advanced parkinson's disease. Neuroscience letters, 683:48-53, 2018.

[77] Alexandre Eusebio, Alek Pogosyan, Shouyan Wang, Bruno Averbeck, Louise Doyle Gaynor, Stephanie Cantiniaux, Tatiana Witjas, Patricia Limousin, Jean-Philippe Azulay, and Peter Brown. Resonance in subthalamo-cortical circuits in parkinson's disease. Brain, 132(8):2139-2150, 2009.

[78] JF Marsden, P Limousin-Dowsey, P Ashby, P Pollak, and P Brown. Subthalamic nucleus, sensorimotor cortex and muscle interrelationships in parkinson's disease. Brain, 124(2):378-388, 2001.

[79] Irene E Harmsen, Nathan C Rowland, Richard A Wennberg, and Andres M Lozano. Characterizing the effects of deep brain stimulation with magnetoencephalography: a review. Brain stimulation, 11(3):481-491, 2018.

[80] Ed Bullmore and Olaf Sporns. The economy of brain network organization. Nature Reviews Neuroscience, 13(5):336, 2012.

[81] Francisco Varela, Jean-Philippe Lachaux, Eugenio Rodriguez, and Jacques Martinerie. The brainweb: phase synchronization and large-scale integration. Nature reviews neuroscience, 2(4):229, 2001. 
[82] Kim TE Olde Dubbelink, Diederick Stoffers, Jan Berend Deijen, Jos WR Twisk, Cornelis J Stam, Arjan Hillebrand, and Henk W Berendse. Resting-state functional connectivity as a marker of disease progression in parkinson's disease: A longitudinal meg study. NeuroImage: Clinical, 2:612-619, 2013.

[83] María Eugenía López, Ricardo Bruña, Sara Aurtenetxe, José Ángel PinedaPardo, Alberto Marcos, Juan Arrazola, Ana Isabel Reinoso, Pedro Montejo, Ricardo Bajo, and Fernando Maestú. Alpha-band hypersynchronization in progressive mild cognitive impairment: a magnetoencephalography study. Journal of Neuroscience, 34(44):14551-14559, 2014.

[84] Eugenia Mamikonyan, Andrew D Siderowf, John E Duda, Marc N Potenza, Stacy Horn, Matthew B Stern, and Daniel Weintraub. Long-term follow-up of impulse control disorders in parkinson's disease. Movement Disorders, 23(1):75-80, 2008.

[85] Werner H Poewe, Olivier Rascol, Niall Quinn, Eduardo Tolosa, Wolfgang $\mathrm{H}$ Oertel, Emilia Martignoni, Markus Rupp, Babak Boroojerdi, SP 515 Investigators, et al. Efficacy of pramipexole and transdermal rotigotine in advanced parkinson's disease: a double-blind, double-dummy, randomised controlled trial. The Lancet Neurology, 6(6):513-520, 2007.

[86] Sanjay P Singh. Magnetoencephalography: basic principles. Annals of Indian Academy of Neurology, 17(Suppl 1):S107, 2014.

[87] Rahul S Desikan, Florent Ségonne, Bruce Fischl, Brian T Quinn, Bradford C Dickerson, Deborah Blacker, Randy L Buckner, Anders M Dale, R Paul Maguire, Bradley T Hyman, et al. An automated labeling system for subdividing the human cerebral cortex on mri scans into gyral based regions of interest. Neuroimage, 31(3):968-980, 2006.

[88] Alexandre Gramfort, Théodore Papadopoulo, Emmanuel Olivi, and Maureen Clerc. Openmeeg: opensource software for quasistatic bioelectromagnetics. Biomedical engineering online, 9(1):45, 2010.

[89] Gerd T Waldhauser, Karl-Heinz T Bäuml, and Simon Hanslmayr. Brain oscillations mediate successful suppression of unwanted memories. Cerebral Cortex, 25(11):4180-4190, 2014.

[90] Madeleine Goodkind, Simon B Eickhoff, Desmond J Oathes, Ying Jiang, Andrew Chang, Laura B Jones-Hagata, Brissa N Ortega, Yevgeniya V Zaiko, Erika L Roach, Mayuresh S Korgaonkar, et al. Identification of a common neurobiological substrate for mental illness. JAMA psychiatry, 72(4):305315, 2015.

[91] Donatus Cyron. Mental side effects of deep brain stimulation (dbs) for movement disorders: The futility of denial. Frontiers in integrative neuroscience, 10:17, 2016. 
[92] Keith A Josephs, Joseph Y Matsumoto, and J Eric Ahlskog. Benign tremulous parkinsonism. Archives of Neurology, 63(3):354-357, 2006.

[93] Fernando H Lopes da Silva, Wouter Blanes, Stiliyan N Kalitzin, Jaime Parra, Piotr Suffczynski, and Demetrios N Velis. Dynamical diseases of brain systems: different routes to epileptic seizures. IEEE Transactions on Biomedical Engineering, 50(5):540-548, 2003.

[94] Alim Louis Benabid, Stephan Chabardes, John Mitrofanis, and Pierre Pollak. Deep brain stimulation of the subthalamic nucleus for the treatment of parkinson's disease. The Lancet Neurology, 8(1):67-81, 2009.

[95] Kenji Sugiyama. Complications of Deep Brain Stimulation. Deep Brain Stimulation for Neurological Disorders: Theoretical Background and Clinical Application, Pages 195-206. Springer, 2014.

[96] Alberto Priori, Guglielmo Foffani, Lorenzo Rossi, and Sara Marceglia. Adaptive deep brain stimulation (adbs) controlled by local field potential oscillations. Experimental neurology, 245:77-86, 2013.

[97] Simon Little, Martijn Beudel, Ludvic Zrinzo, Thomas Foltynie, Patricia Limousin, Marwan Hariz, Spencer Neal, Binith Cheeran, Hayriye Cagnan, James Gratwicke, et al. Bilateral adaptive deep brain stimulation is effective in parkinson's disease. Journal of Neurology, Neurosurgery and Psychiatry, pages jnnp-2015, 2015.

[98] Carmen Camara, Pedro Isasi, Kevin Warwick, Virginie Ruiz, Tipu Aziz, John Stein, and Eduard Bakštein. Resting tremor classification and detection in parkinson's disease patients. Biomedical Signal Processing and Control, 16:88-97, 2015.

[99] Carmen Camara, Kevin Warwick, Ricardo Bruña, Tipu Aziz, Francisco Del Pozo, and Fernando Maestú. A fuzzy inference system for closed-loop deep brain stimulation in parkinson's disease. Journal of medical systems, 39(11):155, 2015.

[100] Romain Carron, Antoine Chaillet, Anton Filipchuk, William Pasillas-Lépine, and Constance Hammond. Closing the loop of deep brain stimulation. Frontiers in systems neuroscience, 7:112, 2013.

[101] Defeng Wu, Kevin Warwick, Zi Ma, Mark N Gasson, Jonathan G Burgess, Song Pan, and Tipu Z Aziz. Prediction of parkinson's disease tremor onset using a radial basis function neural network based on particle swarm optimization. International journal of neural systems, 20(02):109-116, 2010.

[102] Eduard Bakstein, Jonathan Burgess, Kevin Warwick, Virginie Ruiz, Tipu Aziz, and John Stein. Parkinsonian tremor identification with multiple local field potential feature classification. Journal of neuroscience methods, 209(2):320330, 2012. 
[103] Ishita Basu, Daniel Graupe, Daniela Tuninetti, Pitamber Shukla, Konstantin V Slavin, Leo Verhagen Metman, and Daniel M Corcos. Pathological tremor prediction using surface electromyogram and acceleration: potential use in 'on-off'demand driven deep brain stimulator design. Journal of neural engineering, 10(3):036019, 2013.

[104] J Hirschmann, JM Schoffelen, A Schnitzler, and MAJ Van Gerven. Parkinsonian rest tremor can be detected accurately based on neuronal oscillations recorded from the subthalamic nucleus. Clinical Neurophysiology, 128(10):2029-2036, 2017.

[105] M. Bacher, E. Scholz, and H.C. Diener. 24 hour continuous tremor quantification based on EMGG recording. Electroencephalography and Clinical Neurophysiology, 72(2):176 - 183, 1989.

[106] S. Spieker, A. Boose, S. Breit, and J. Dichgans. Long-term measurement of tremor. Movement Disorders, 13(S3):81-84, 1998.

[107] K. E. Norman, R. E., and A. Beuter. The measurement of tremor using a velocity transducer: comparison to simultaneous recordings using transducers of displacement, acceleration and muscle activity. Journal of Neuroscience Methods, 92(1-2):41 - 54, 1999.

[108] G. Deuschl, P. Bain, and M. Brin. Consensus statement of the movement disorder society on tremor. Movement Disorders, 13(S3):2-23, 1998.

[109] A. E. Lang and C. Zadikoff. Handbook of Essential Tremor and other Tremors Disorders. Taylor and Francis, 2005.

[110] J. Jankovic and E. Tolosa. Parkinson's Disease and Movement Disorders. Lippincott Williams \& Wilkins, 2007.

[111] J. W. Lance, R. S. Schwab, and E. A. Peterson. Action tremors and the cogwheel phenomenon in parkinson's disease. Brain, 86(1):95-110, 1963.

[112] E. D Louis, G. Levy, L. J. Cote, H. Mejia, S. Fahn, and K. Marder. Clinical correlates of action tremor in parkinson disease. Archives of Neurology, 58(10):1630-1634, 2001.

[113] W. C. Koller, B. Vter-Overfield, and R. Barter. Tremors in early parkinson disease. Clinical neuropharmacology, 12(4):293-297, 1989.

[114] G. Deuschl, J. Raethjen, R. Baron, M. Lindenmann, H. Wilms, and P. Krack. The pathophysiology of parkinsonian tremor: a review. Journal of neurology, 247(5):V33-V48, 2000.

[115] R. Wenzelburger, J. Raethjen, K. Loffler, H. Stolze, M Illert, and G. Deuschl. Kinetic tremor in a reach-to-grasp movement in parkinson's disease. Movement Disorder, 15(6):1084-1094, 2000. 
[116] A. Beuter, E. Barbo, R. Rigal, and P. J. Blanchet. Characterization of subclinical tremor in parkinson's disease. Movement Disorders, 20(8):945-950, 2005.

[117] A. Hristova, K. Lyons, A. Troster, R. Pahwa, S. Wilkinson, and W. C. Koller. Effect and time course of deep brain stimulation of the globus pallidus and subthalamus on motor features of parkinson's disease. Clinical neuropharmacology, 23(4):208-211, 2000.

[118] J. G. Burgess, K. Warwick, V. Ruiz, M. Gasson, T.Z. Aziz, J-S. Brittain, and J. Stein. Identifying tremor-related characteristics of basal ganglia nuclei during movement in the parkinsonian patient. Parkinsonism \& Related Disorders, 16(10):671-675, 2010.

[119] P. Brown and D. Williams. Basal ganglia local field potential activity: Character and functional significance in the human. In Clinical Neurophysiology, Official Journal of the IFCN, 116(11):1388-2457, 2005.

[120] E. Bakstein, J. Burgess, K. Warwick, V. Ruiz, T. Aziz, and J. Stein. Parkinsonian tremor identification with multiple local field potential feature classification. Journal of Neuroscience Methods, 209(2):320 - 330, 2012.

[121] A. K Jain and R. Dubes. Algorithms for Clustering Data. Prentice Hall, 1988.

[122] B. Yao, S. Salenius, G. H. Yue, R. W. Brown, and J. Z. Liu. Effects of surface emg rectification on power and coherence analyses: an eeg and meg study. Journal of neuroscience methods, 159(2):215-223, 2007.

[123] L. J Myers, M. Lowery, M. O'Malley, C. L. Vaughan, C. Heneghan, A. St. Clair Gibson, Y. X. R. Harley, and R. Sreenivasan. Rectification and non-linear pre-processing of emg signals for cortico-muscular analysis. Journal of Neuroscience Methods, 124(2):157 - 165, 2003.

[124] R. Quian Quiroga, A. Kraskov, T. Kreuz, and P. Grassberger. Performance of different synchronization measures in real data: a case study on electroencephalographic signals. Physical Review E, 65(4):041903, 2002.

[125] J.P. Lachaux, E. Rodriguez, J. Martinerie, F. J Varela, et al. Measuring phase synchrony in brain signals. Human brain mapping, 8(4):194-208, 1999.

[126] J. J. Buckley and E. Eslami. An introduction to fuzzy logic and fuzzy sets, volume 13. Springer Science \& Business Media, 2002.

[127] M. N. Gasson, S. Y. Wang, T. Z. Aziz, J. F. Stein, and K. Warwick. Towards a demand driven deep-brain stimulator for the treatment of movement disorders. 2005.

[128] J. Jantzen. Design Of Fuzzy Controllers. (98):1 - 28, 1998.

[129] C. Camara, P. Isasi, K. Warwick, V. Ruiz, T. Aziz, J. Stein, and E. Bakštein. Resting tremor classification and detection in parkinson's disease patients. Biomedical Signal Processing and Control, 16(0):88 - 97, 2015. 
[130] Andrea A Kühn, Andreas Kupsch, Gerd-Helge Schneider, and Peter Brown. Reduction in subthalamic 8-35 hz oscillatory activity correlates with clinical improvement in parkinson's disease. European Journal of Neuroscience, 23(7):1956-1960, 2006.

[131] Moran Weinberger, William D Hutchison, and Jonathan O Dostrovsky. Pathological subthalamic nucleus oscillations in pd: can they be the cause of bradykinesia and akinesia? Experimental neurology, 219(1):58-61, 2009.

[132] FU Hohlefeld, C Huchzermeyer, J Huebl, G-H Schneider, G Nolte, C Brücke, T Schönecker, AA Kühn, G Curio, and VV Nikulin. Functional and effective connectivity in subthalamic local field potential recordings of patients with parkinson's disease. Neuroscience, 250:320-332, 2013.

[133] C Camara, K Warwick, R Bruña, T Aziz, and E Pereda. Closed-loop deep brain stimulation based on a stream-clustering system. Expert Systems with Applications, 126:187-199, 2019.

[134] Fernando Lopes Da Silva, Wouter Blanes, Stiliyan N Kalitzin, Jaime Parra, Piotr Suffczynski, and Demetrios N Velis. Epilepsies as dynamical diseases of brain systems: basic models of the transition between normal and epileptic activity. Epilepsia, 44:72-83, 2003.

[135] Reik V Donner, Yong Zou, Jonathan F Donges, Norbert Marwan, and Jürgen Kurths. Recurrence networks-a novel paradigm for nonlinear time series analysis. New Journal of Physics, 12(3):033025, 2010.

[136] Narayan Puthanmadam Subramaniyam, Jari Hyttinen, Nicholas G Hatsopoulos, and Kazutaka Takahashi. Recurrence network analysis of wide band oscillations of local field potentials from the primary motor cortex reveals rich dynamics. In Neural Engineering (NER), 2015 7th International IEEE/EMBS Conference on, pages 960-963. IEEE, 2015.

[137] NP Subramaniyam, Jonathan F Donges, and J Hyttinen. Signatures of chaotic and stochastic dynamics uncovered with $\varepsilon$-recurrence networks. In Proc. $R$. Soc. A, volume 471, page 20150349. The Royal Society, 2015.

[138] Floris Takens. Detecting strange attractors in turbulence. In Dynamical systems and turbulence, Warwick 1980, pages 366-381. Springer, 1981.

[139] Andrew M Fraser and Harry L Swinney. Independent coordinates for strange attractors from mutual information. Physical review A, 33(2):1134, 1986.

[140] Holger Kantz and Thomas Schreiber. Nonlinear time series analysis, volume 7. Cambridge university press, 2004.

[141] Robert Shaw. The dripping faucet as a model chaotic system. 1984. 
[142] Matthew B Kennel, Reggie Brown, and Henry DI Abarbanel. Determining embedding dimension for phase-space reconstruction using a geometrical construction. Physical review A, 45(6):3403, 1992.

[143] Reik V Donner, Michael Small, Jonathan F Donges, Norbert Marwan, Yong Zou, Ruoxi Xiang, and Jürgen Kurths. Recurrence-based time series analysis by means of complex network methods. International Journal of Bifurcation and Chaos, 21(04):1019-1046, 2011.

[144] Norbert Marwan, Jonathan F Donges, Yong Zou, Reik V Donner, and Jürgen Kurths. Complex network approach for recurrence analysis of time series. Physics Letters A, 373(46):4246-4254, 2009.

[145] Small Michael. Applied nonlinear time series analysis: applications in physics, physiology and finance, volume 52. World Scientific, 2005.

[146] Thomas Schank and Dorothea Wagner. Approximating clustering-coefficient and transitivity. Universität Karlsruhe, Fakultät für Informatik, 2004.

[147] Mark EJ Newman. Assortative mixing in networks. Physical review letters, 89(20):208701, 2002.

[148] Nello Cristianini, John Shawe-Taylor, et al. An introduction to support vector machines and other kernel-based learning methods. Cambridge university press, 2000.

[149] Carmen Camara, Pedro Peris-Lopez, and Juan E Tapiador. Security and privacy issues in implantable medical devices: A comprehensive survey. Journal of biomedical informatics, 55:272-289, 2015.

[150] Karl J Friston. Functional and effective connectivity in neuroimaging: a synthesis. Human brain mapping, 2(1-2):56-78, 1994.

[151] Marcel Antonius Johannes Lourens, Hil Gaétan Ellart Meijer, MF Contarino, P Van Den Munckhof, PR Schuurman, Stephanus A van Gils, and LJ Bour. Functional neuronal activity and connectivity within the subthalamic nucleus in parkinson's disease. Clinical neurophysiology, 124(5):967-981, 2013.

[152] Alfons Schnitzler and Joachim Gross. Normal and pathological oscillatory communication in the brain. Nature reviews neuroscience, 6(4):285, 2005.

[153] Peter Brown and David Williams. Basal ganglia local field potential activity: character and functional significance in the human. Clinical neurophysiology, 116(11):2510-2519, 2005.

[154] Peter Brown, Paolo Mazzone, Antonio Oliviero, Maria Grazia Altibrandi, Fabio Pilato, Pietro A Tonali, and Vincenzo Di Lazzaro. Effects of stimulation of the subthalamic area on oscillatory pallidal activity in parkinson's disease. Experimental neurology, 188(2):480-490, 2004. 
[155] Brett Wingeier, Tom Tcheng, Mandy Miller Koop, Bruce C Hill, Gary Heit, and Helen M Bronte-Stewart. Intra-operative stn dbs attenuates the prominent beta rhythm in the stn in parkinson's disease. Experimental neurology, 197(1):244-251, 2006.

[156] Wassilios Meissner, Arthur Leblois, David Hansel, Bernard Bioulac, Christian E Gross, Abdelhamid Benazzouz, and Thomas Boraud. Subthalamic high frequency stimulation resets subthalamic firing and reduces abnormal oscillations. Brain, 128(10):2372-2382, 2005.

[157] Moran Weinberger, Neil Mahant, William D Hutchison, Andres M Lozano, Elena Moro, Mojgan Hodaie, Anthony E Lang, and Jonathan O Dostrovsky. Beta oscillatory activity in the subthalamic nucleus and its relation to dopaminergic response in parkinson's disease. Journal of neurophysiology, 96(6):3248-3256, 2006.

[158] Ron Levy, William D Hutchison, Andres M Lozano, and Jonathan O Dostrovsky. High-frequency synchronization of neuronal activity in the subthalamic nucleus of parkinsonian patients with limb tremor. Journal of Neuroscience, 20(20):7766-7775, 2000.

[159] Gunther Deuschl, Jan Raethjen, Ralph Baron, Michael Lindemann, Henrik Wilms, and Paul Krack. The pathophysiology of parkinsonian tremor: a review. Journal of neurology, 247(5):V33-V48, 2000.

[160] Mark D Bevan, Jeremy F Atherton, and Jérôme Baufreton. Cellular principles underlying normal and pathological activity in the subthalamic nucleus. Current opinion in neurobiology, 16(6):621-628, 2006.

[161] Andrea A Kühn, Thomas Trottenberg, Anatol Kivi, Andreas Kupsch, GerdHelge Schneider, and Peter Brown. The relationship between local field potential and neuronal discharge in the subthalamic nucleus of patients with parkinson's disease. Experimental neurology, 194(1):212-220, 2005.

[162] A Priori, G Foffani, A Pesenti, F Tamma, AM Bianchi, M Pellegrini, M Locatelli, KA Moxon, and RM Villani. Rhythm-specific pharmacological modulation of subthalamic activity in parkinson's disease. Experimental neurology, 189(2):369-379, 2004.

[163] S Marceglia, G Foffani, AM Bianchi, G Baselli, F Tamma, M Egidi, and A Priori. Dopamine-dependent non-linear correlation between subthalamic rhythms in parkinson's disease. The Journal of physiology, 571(3):579-591, 2006.

[164] Ian H Witten, Eibe Frank, Mark A Hall, and Christopher J Pal. Data Mining: Practical machine learning tools and techniques. Morgan Kaufmann, 2016.

[165] Dragana Miljkovic, Darko Aleksovski, Vid Podpečan, Nada Lavrač, Bernd Malle, and Andreas Holzinger. Machine learning and data mining methods for managing parkinson's disease. In Machine Learning for Health Informatics, pages 209-220. Springer, 2016. 
[166] Albert Bifet, Ricard Gavaldà, Geoff Holmes, and Bernhard Pfahringer. Machine Learning for Data Streams: with Practical Examples in MOA. MIT Press, 2018.

[167] Hardy Kremer, Philipp Kranen, Timm Jansen, Thomas Seidl, Albert Bifet, Geoff Holmes, and Bernhard Pfahringer. An effective evaluation measure for clustering on evolving data streams. In Proceedings of the 17th ACM SIGKDD international conference on Knowledge discovery and data mining, pages 868-876. ACM, 2011.

[168] Emo Welzl. Smallest enclosing disks (balls and ellipsoids). In New results and new trends in computer science, pages 359-370. Springer, 1991.

[169] Bernd Gärtner. Fast and robust smallest enclosing balls. In European Symposium on Algorithms, pages 325-338. Springer, 1999.

[170] A.Bifet. Stream Clustering - Github, 2018. accessed August 20, 2018.

[171] Mohamed Medhat Gaber, Arkady Zaslavsky, and Shonali Krishnaswamy. Mining data streams: a review. ACM Sigmod Record, 34(2):18-26, 2005.

[172] Joao Gama. Knowledge discovery from data streams. CRC Press, 2010.

[173] David Arthur and Sergei Vassilvitskii. k-means++: The advantages of careful seeding. In Proceedings of the eighteenth annual ACM-SIAM symposium on Discrete algorithms, pages 1027-1035. Society for Industrial and Applied Mathematics, 2007.

[174] Andrew McCallum, Kamal Nigam, and Lyle H Ungar. Efficient clustering of high-dimensional data sets with application to reference matching. In Proceedings of the sixth ACM SIGKDD international conference on Knowledge discovery and data mining, pages 169-178. ACM, 2000.

[175] ME McNeely, T Hershey, MC Campbell, SD Tabbal, M Karimi, JM Hartlein, HM Lugar, FJ Revilla, JS Perlmutter, and GM Earhart. Effects of deep brain stimulation of dorsal versus ventral subthalamic nucleus regions on gait and balance in parkinson's disease. Journal of Neurology, Neurosurgery and Psychiatry, 82(11):1250-1255, 2011.

[176] Joel S Perlmutter and Jonathan W Mink. Deep brain stimulation. Annual Review of Neuroscience, 29:229-257, 2006.

[177] Abdelhamid Benazzouz, Christian Gross, Jean Féger, Thomas Boraud, and Bernard Bioulac. Reversal of rigidity and improvement in motor performance by subthalamic high-frequency stimulation in mptp-treated monkeys. European Journal of Neuroscience, 5(4):382-389, 1993.

[178] AL Benabid, P Pollak, Ch Gross, D Hoffmann, A Benazzouz, DM Gao, A Laurent, M Gentil, and J Perret. Acute and long-term effects of subthalamic nucleus stimulation in parkinson's disease. Stereotactic and functional neurosurgery, 62(1-4):76-84, 1994. 
[179] Medtronic Activa PC+S deep brain neurostimulator.

https:

//medtronicmediacap.gcs-web.com/new-medtronic-deep-

brain-stimulation-system-first-sense-and-record-brainactivity-while. Accessed: 2019-05-20.

[180] Nir Grossman, David Bono, Nina Dedic, Suhasa B Kodandaramaiah, Andrii Rudenko, Ho-Jun Suk, Antonino M Cassara, Esra Neufeld, Niels Kuster, LiHuei Tsai, et al. Noninvasive deep brain stimulation via temporally interfering electric fields. Cell, 169(6):1029-1041, 2017.

[181] Laurie Pycroft, Sandra G Boccard, Sarah LF Owen, John F Stein, James J Fitzgerald, Alexander L Green, and Tipu Z Aziz. Brainjacking: implant security issues in invasive neuromodulation. World neurosurgery, 92:454-462, 2016. 